\title{
Plaque angiogenesis and lymphangiogenesis : can small vessels influence atherosclerosis?
}

Citation for published version (APA):

Rademakers, T. (2013). Plaque angiogenesis and lymphangiogenesis : can small vessels influence atherosclerosis? [Doctoral Thesis, Maastricht University]. Uitgeverij BOXPress. https://doi.org/10.26481/dis.20130626tr

Document status and date:

Published: 01/01/2013

DOI:

10.26481/dis.20130626tr

Document Version:

Publisher's PDF, also known as Version of record

\section{Please check the document version of this publication:}

- A submitted manuscript is the version of the article upon submission and before peer-review. There can be important differences between the submitted version and the official published version of record.

People interested in the research are advised to contact the author for the final version of the publication, or visit the DOI to the publisher's website.

- The final author version and the galley proof are versions of the publication after peer review.

- The final published version features the final layout of the paper including the volume, issue and page numbers.

Link to publication

\footnotetext{
General rights rights.

- You may freely distribute the URL identifying the publication in the public portal. please follow below link for the End User Agreement:

www.umlib.nl/taverne-license

Take down policy

If you believe that this document breaches copyright please contact us at:

repository@maastrichtuniversity.nl

providing details and we will investigate your claim.
}

Copyright and moral rights for the publications made accessible in the public portal are retained by the authors and/or other copyright owners and it is a condition of accessing publications that users recognise and abide by the legal requirements associated with these

- Users may download and print one copy of any publication from the public portal for the purpose of private study or research.

- You may not further distribute the material or use it for any profit-making activity or commercial gain

If the publication is distributed under the terms of Article $25 \mathrm{fa}$ of the Dutch Copyright Act, indicated by the "Taverne" license above, 


\section{Plaque angiogenesis and}

\section{lymphangiogenesis: can small}

\section{vessels influence atherosclerosis?}


Layout: Timo Rademakers

Cover design: Timo Rademakers

(Cover illustration depicts a midwinter forest resembling angiogenesis)

Printed by: Proefschriftmaken.nl || Uitgeverij BOXPress

Published by: Uitgeverij BOXPress, Oisterwijk

ISBN: 978-90-8891-612-0

(c) 2013 Timo Rademakers, Maastricht 


\section{Plaque angiogenesis and \\ lymphangiogenesis: can small \\ vessels influence atherosclerosis?}

\section{PROEFSCHRIFT}

Ter verkrijging van de graad van doctor aan de Universiteit Maastricht, op gezag van de Rector Magnificus, Prof. Dr. L.L.G. Soete volgens het besluit van het College van Decanen, in het openbaar te verdedigen op woensdag 26 juni 2013 om 10.00 uur door

Timo Rademakers

geboren 03 juli 1985 te Brunssum 


\section{Promotores}

Prof. dr. E.A.L. Biessen

Prof. dr. M.A.M.J. van Zandvoort

\section{Co-promotor}

Dr. S. Heeneman

\section{Beoordelingscommissie}

Prof. Dr. F.C.S. Ramaekers (voorzitter)

Prof. Dr. A.B. Houtsmuller (Erasmus MC, Rotterdam)

Dr. M.E. Kooi

Prof. Dr. R.J. van Oostenbrugge

Prof. Dr. C.J.M. de Vries (AMC, Amsterdam)

Financial support by the Netherlands Heart Foundation for the publication of this thesis is gratefully acknowledged.

Nederlandse $\overbrace{\text { Hartstichting }}$ 




\section{Contents}

Chapter 1 General Introduction

Chapter 2 Multiphoton laser scanning microscopy in atherosclerosis research

Chapter 3 Plaque-associated vasa vasorum in aged apolipoprotein-E deficient mice exhibit pro-atherogenic functional features in vivo

Chapter 4 Flow-dependent redistribution of endothelial junctional adhesion molecule- $\mathrm{A}$ in atherosclerosis causes increased leukocyte recruitment

Chapter 5 VE-cadherin as a potential mediator of microvascular permeability in atherosclerosis?

Chapter 6 Angiogenic and lymphangiogenic responses in atherosclerosis development - a genomics approach to identify new regulators of plaque vascularization

Chapter 7 Increased adventitial lymphatic capillaries in atherosclerosis: a path for T cell trafficking?

Samenvatting

Curriculum Vitae 


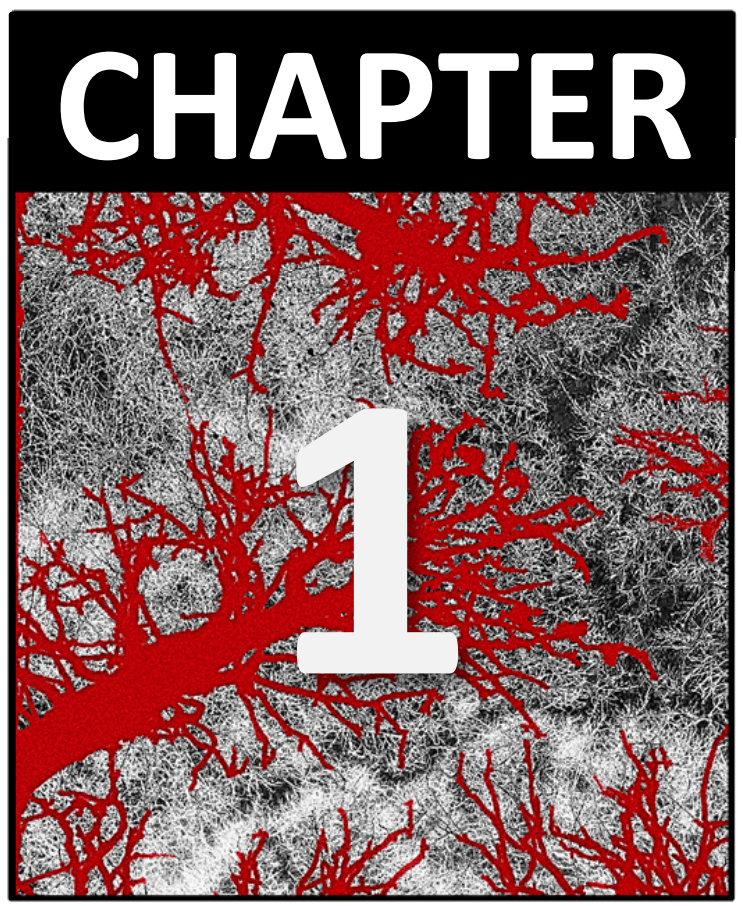


General Introduction 
Currently, cardiovascular diseases are the leading cause of mortality worldwide, accounting for about 17 million global deaths every year, with about 7.3 million deaths caused by coronary heart disease or acute myocardial infarction (AMI), and 6.2 million deaths due to stroke ${ }^{1-4}$. This number is expected to increase further in the next decades ${ }^{4-6}$. The acute clinical manifestations of cardiovascular diseases (i.e. AMI and stroke) are mainly caused by rupture of advanced atherosclerotic plaques, rendering atherosclerosis the major cause for cardiovascular disease ${ }^{3}$.

Atherosclerosis is a progressive vascular disease, characterized by accumulation of lipids and cellular and fibrous constituents in the arterial tree (Figure 1). The onset of atherosclerosis in humans can be as early as childhood, when lesions called fatty streaks develop at sites of disturbed blood flow ${ }^{7-10}$. At these sites, e.g. the coronary arteries, the aortic arch, and the bifurcation of the carotid artery, the endothelial layer is susceptible for the entry of lipids, allowing influx of low density lipoprotein (LDL) into the subendothelial space, where it subsequently undergoes modifications like oxidation (oxLDL). As a response to this lipid influx, immune cells will be recruited to remove these lipids. This is facilitated by an increase in adhesion molecules on the endothelium and a rise in local cytokines and chemokines at the site of inflammation ${ }^{11}$, allowing recruitment of inflammatory cells to the subendothelial space. Primarily monocytes will be attracted, which, in the subendothelial layer, differentiate into macrophages. After lipid uptake, these macrophages develop into foam cells and are rendered immobile, giving rise to a gradual accumulation of macrophages in the subendothelial layer, and a subsequent chronic inflammatory response, involving recruitment of more inflammatory cell types (e.g. T cells, B cells, neutrophils, dendritic cells (DCs)) towards the atherosclerotic lesion reviewed in 12-15.

With time, the inflammatory milieu and local inflammatory stimuli will induce local apoptosis and necrosis of local inflammatory cells. Lack of efferocytosis, i.e. the clearance of apoptotic cells, as well as the increased necrosis and continued accumulation of lipids and inflammatory cells, will cause plaque progression towards a more advanced plaque phenotype ${ }^{16-18}$. In these advanced lesions, vascular smooth muscle cells (VSMCs) are recruited from the medial layer in response to chemotactic signals and form a collagenrich fibrous cap, maintaining vascular integrity and plasticity by preventing the plaque from rupturing and releasing the necrotic and lipid-rich plaque material 


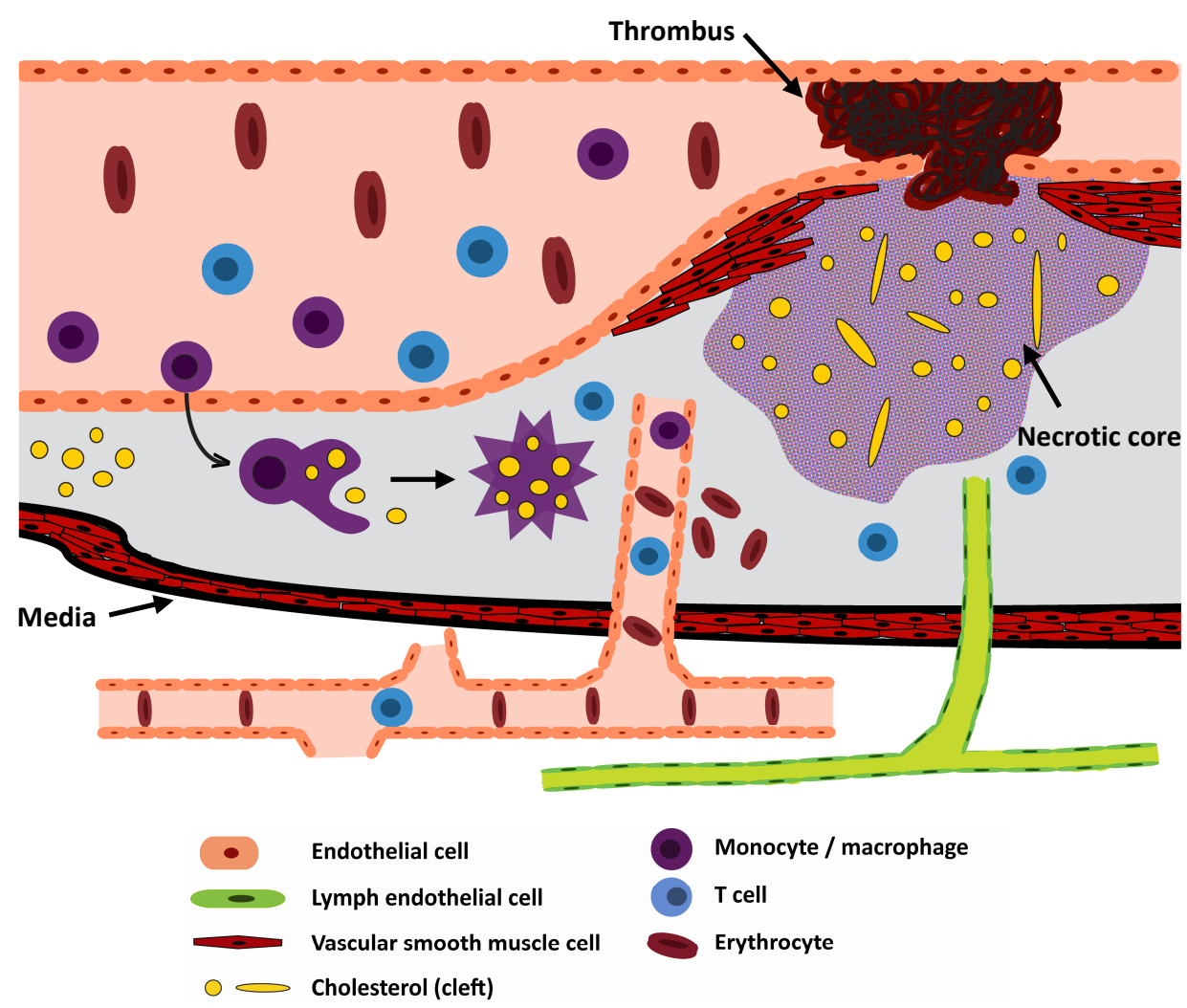

Figure 1. Schematic representation of atherosclerosis development with time. Lesion formation is initiated by accumulation of lipids in the subendothelial space (between the EC layer and the medial layer) at sites of disturbed flow. After local modification of the lipids in the subendothelial space, e.g. oxidation of $L D L$ (ox $L D L)$, an immune response is elicited to clear these lipids. Monocytes enter from the blood, differentiate into macrophages and take up the modified lipids, giving rise to foam cell formation. Additional inflammatory cells (e.g. T cells, B cells and DCs) are recruited, potentiating the chronic inflammatory response. With time, an advanced lesions consisting of a lipid-rich necrotic core (light purple) and a fibrous, collagen-and VSMC-rich cap is formed. Production of proteases can destabilize the fibrous cap, potentially causing plaque rupture and consequent thrombotic events. During the progression of lesion formation, an expansion of adventitial vasa vasorum and lymph capillaries has been recorded, accompanied by ingress of both vessel types into the lesion. Expansion of blood and lymph vessels has been associated with a more advanced plaque stage. Figure based on Sanz et al ${ }^{19}$. 


\section{CHAPTER 1}

into the circulation. This lesion type is considered to be a stable advanced lesion. However, production of several types of proteases, e.g. matrixdegrading metalloproteases (MMPs), by inflammatory cells, will destabilize the fibrous cap, allowing the plaque to evolve to an unstable, vulnerable plaque phenotype ${ }^{20}$. Upon rupture, the necrotic core with its pro-thrombotic components will come into contact with the blood, causing thrombus formation, potentially causing occlusion of coronary arteries (acute myocardial infarction) or occlusion of cerebral arteries (stroke). Two main focus points of atherosclerosis research have been lipid metabolism and uptake, and inflammation in the atherosclerotic lesion. Although these processes are of key importance, other processes have been gaining more and more interest, including plaque hypoxia which gives rise to expansion of local microcirculation, and involvement of the lymphatic system ${ }^{21,22}$. Definitely, vulnerable plaques are not merely characterized by the presence of a large lipid-laden necrotic core underlying a thin fibrous cap, and abundant inflammatory cell deposits in the adventitia and the shoulder regions of the plaque ${ }^{23}$. Atherosclerotic plaques are to a large extent hypoxic ${ }^{24}$, providing a basis for an angiogenic response. Indeed, multiple human studies have shown that more advanced lesions are generally heavily vascularized, and the extent of vascularization has been shown to correlate with progression stage, inflammatory content of the plaque, and plaque stability ${ }^{25}$, reviewed in ${ }^{26}$. More recently, evidence has been presented showing an increase in lymphatic capillaries in both early and advanced lesions ${ }^{21,27}$, as will be discussed in more detail later. 


\section{Atherosclerosis and angiogenesis}

The concept of an angiogenic response occurring during plaque development is not new. It was already in 1876 that Köster suggested that (advanced) atherosclerotic lesions are associated with an increased density in vasa vasorum ${ }^{28}$. The vasa vasorum is the adventitial plexus of microvessels surrounding the larger arteries $\left(>0.5 \mathrm{~mm}\right.$ in diameter $\left.{ }^{29}\right)$ that provides the medial and adventitial layer of arteries with oxygen and nutrients and removes waste products. However, it was not until three decades ago that plaque neovascularization regained interest, which led to a better understanding of its involvement in atherosclerosis ${ }^{26}$.

\section{General background}

Angiogenesis is defined as the sprouting of new blood vessels from pre-existing blood vessels, and is not to be confused with arteriogenesis (functional dilatation and maturation of pre-formed collaterals) or vasculogenesis (de novo formation of blood vessels from progenitor cells). This process of angiogenesis is of great physiological importance during embryogenesis, development, and wound healing, but is involved in a plethora of pathological conditions as well, such as oncology, rheumatoid arthritis, vascular complications in diabetes like retinopathies, endometriosis, and atherosclerosis. The normal, physiological angiogenic process can be divided in several stages, as was reviewed extensively ${ }^{30-33}$.

Shortly, in a hypoxic or inflammatory environment in which angiogenic mediators are secreted, nearby endothelial cells (ECs) are activated (Figure 2). This is followed by (i) detachment of pericytes from the vessel wall by angiopoietin-2 (Ang-2) and MMPs and (ii) loosening of the endothelial junctions by vascular endothelial growth factors (VEGFs) and other vascular growth factors, causing vessel dilatation and increasing vascular permeability ${ }^{33,34}$. Due to this increased permeability, plasma proteins can enter the extravascular space, and provide a basis for the second step, the proliferation and migration of ECs. To this end, two types of ECS can be distinguished, namely the tip cell and the stalk cell. Tip cells are equipped with filopodia, which they use to sense gradients of angiogenic (guidance) molecules like VEGF and ephrins to migrate towards the site of hypoxia or inflammation. The stalk cells follow the lead of the tip cells, and are the cells 
which elongate the newly forming vessel by repeated cellular division ${ }^{35}$ (under control of e.g. NOTCH , WNT and several growth factors), and allow formation of a lumen ${ }^{36}$ (requiring amongst others VE-cadherin, VEGFs and their receptors (VEGFRs), CD105, CD34, junctional adhesion molecule-A (JAM-A) ${ }^{37}$ ). From here, an immature vessel is formed and the surrounding extracellular matrix (ECM) is reorganized to support this immature vessel, and form a new basement membrane (stimulated by protease inhibitors like tissue inhibitors of metalloproteinases (TIMPs)). The final step in the angiogenic process is vessel maturation, in which pericytes are recruited to cover the immature vessels. This step, which is also described as the "quiescent phalanx resolution" ${ }^{34}$, is highly dependent on angiopoietin-1 (Ang-1), transforming growth factor- $\beta$ (TGF- $\beta$ ) and platelet-derived growth factor B (PDGF-B). In addition, the new blood vessel may undergo an additional step of arterio-venous differentiation, governed by ephrins and NOTCH signaling ${ }^{30,34}$. Arterio-venous differentiation allows local adaptation and will influence, e.g. permeability, by changing the intra-endothelial junctions or pericyte coverage rate.

During pathological angiogenesis, the development of new (micro)vessels is aberrant. Two major types of abnormalities can be distinguished. The first comprises of aberrations in guidance and proliferation, causing an unorganized growth of the newly formed vessels with excessive branching and enlarged luminal diameters. The second type of aberrations involves atypical maturation, characterized by e.g. basement membrane detachment, lack of pericyte coverage, junctional abnormalities, causing functional defects and a 'leaky' phenotype. Both types of aberrations are wellknown from tumor angiogenesis, but are also present in other pathologies, including atherosclerosis ${ }^{32,38,39}$. 


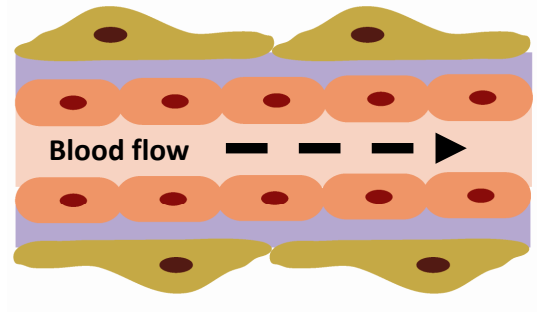

A

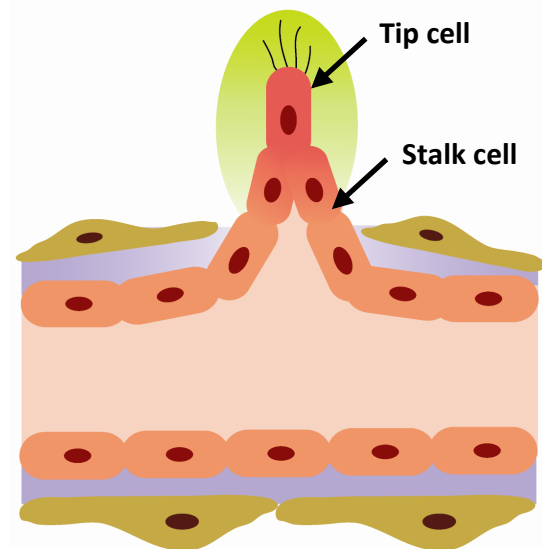

C
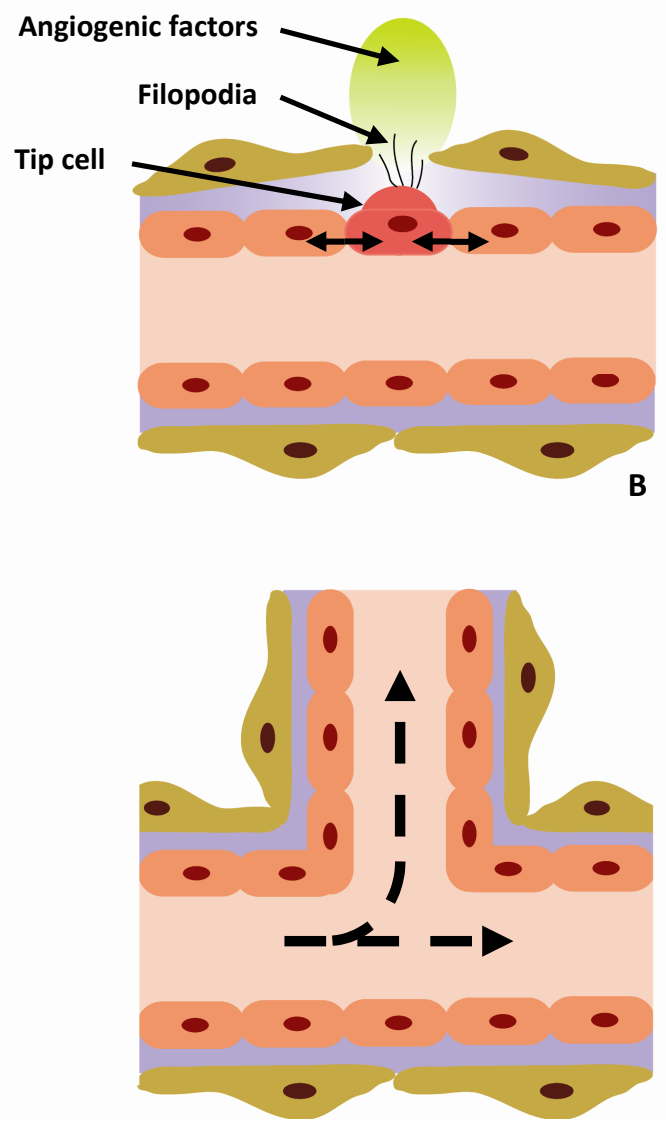

D

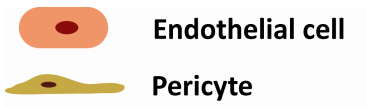

\section{Basement membrane}

Angiogenic gradient

Figure 2. Schematic representation of the angiogenic process. Upon a hypoxic stimulus, the quiescent vessel $(A)$ is activated, inducing degradation of the basement membrane, detachment of pericytes from the vessel wall, vessel dilatation, and loosening of the endothelial junctions causing increased in vascular permeability (B). Plasma proteins enter the extravascular space, facilitating proliferation and migration of endothelial cells. Initially, tip cells (red), equipped with filopodia, sense gradients of angiogenic (guidance) molecules to migrate towards the site of hypoxia or inflammation. The stalk cells follow the lead of the tip cells and elongate the newly forming vessel by repeated cellular division (C). The surrounding extracellular matrix (ECM) is reorganized to support the immature vessel, and a new basement membrane is formed. Finally, pericytes are recruited to cover and stabilize the immature vessel, forming a stable, mature vessel (D). Figure based on Carmeliet et al ${ }^{34}$. 


\section{Angiogenesis in atherosclerosis}

As described earlier, the first description of adventitial microvasculature being present in atherosclerotic lesions dates back to the late 1800s, when Köster noted an increased vasa vasorum density in humans. In the 1930s, Paterson described intimal vascularization of human atherosclerotic coronary arteries ${ }^{40}$. After that, it was not until the 1980s that plaque vascularization regained interest ${ }^{41,42}$. Kamat et al were among the first to study localization of plaque neovascularization and performed the first actual quantitative histological analysis on human coronary arteries, and could confirm extensive vasa vasorum expansion, although intraplaque vascularization was undetectable ${ }^{43}$. Later studies led to further classification and quantification of plaque neovascularization during atherosclerosis progression ${ }^{44-48}$. Overall, there is a clear association between expansion of microvessels and plaque progression, both in the adventitia and the intima ${ }^{26}$.

Not only the expansion of plaque microvessels points towards a role of angiogenesis in atherosclerotic lesions. Others have focused on the angiogenic response, and were able to show that human plaque material exhibits angiogenic properties ${ }^{49}$. Since then, it has been established that well-known angiogenic factors are present in atherosclerotic lesions, including members of the VEGF and VEGFR family ${ }^{50-52}$, placental growth factor (PIGF) ${ }^{53,54}$, and Ang-1 and Ang- $2^{55}$, as well as factors associated with the angiogenic process, e.g. MMPs ${ }^{56,57}$. Furthermore, several cell types within the atherosclerotic plaque may express these pro-angiogenic factors, like mast cells ${ }^{58-60}$ and macrophages 22, 61, 62. More importantly, it was shown that atherosclerotic lesions are hypoxic due to an increased physical distance to the lumen or the vasa vasorum - greater than the diffusion distance of oxygen and nutrients (approximately 100-250 $\mu \mathrm{m}$ ) ${ }^{63}$ - and an increased metabolic activity of inflammatory cells inside the lesion ${ }^{24,26}$. Hypoxia would be the logical trigger for the angiogenic process.

The important question that arises is whether angiogenesis in human atherosclerosis is a beneficial or detrimental event. Over the years, atherosclerotic plaque neovascularization has been attributed a dual role, as also described by Moreno et $a l^{22}$. On the one hand, it has been suggested that (initial) angiogenesis would be a means to resolve the atherosclerotic lesion. Not only would it resolve plaque hypoxia, but formation of new microvessels in 
atherosclerosis may guide efflux of macrophages and T cells, or alternatively allow ingress of anti-inflammatory cell types, as Moreno et al suggest from their data ${ }^{22}$. Also, plaque angiogenesis may be involved in removal of intimal LDL when the systemic LDL concentration is lower than intimal LDL concentrations ${ }^{64}$. This could be the case during statin treatment, which e.g. in the SATURN study was shown to induce plaque regression ${ }^{65}$. Though some experimental studies in larger animal models are in support of a beneficial function of plaque neovascularization ${ }^{66-70}$, this point of view is still debated, and should be substantiated in vivo.

On the other hand, a more substantial amount of evidence suggests that plaque angiogenesis is detrimental. As mentioned earlier, there is a strong association between enhanced vascularization and the progression from initial towards advanced, vulnerable lesions in human atherosclerosis ${ }^{26}$. The current general hypothesis is that local hypoxia would induce release of angiogenic factors inside the lesion, inducing ingrowth of microvessels into the lesion, after initial expansion of the vasa vasorum ${ }^{71-73}$. A part of the intraplaque microvessels may however derive from sprouting from the luminal endothelium ${ }^{74-76}$. Due to the inflammatory environment, the endothelium of the plaque microvessels is likely to attain a leaky phenotype, characterized by impaired functionality and leakiness, i.e. open intra-endothelial junctions, basement membrane detachment and absent pericyte coverage, as was described after ultrastructural analysis using electron microscopy (EM) ${ }^{77}$. Such leaky microvessels would in turn favor the influx of circulating erythrocytes, leukocytes, and blood platelets into the plaque ${ }^{72,}{ }^{78-80}$. In fact, plaque microvessels have already been associated with accumulation of a myriad of inflammatory cells, as well as lipoproteins and albumin, as reviewed by Sluimer et $a^{26}$ and may thus promote further plaque expansion, destabilization, and thrombogenicity. Alternatively, intraplaque microvessels may weaken the biomechanical integrity of the plaque, particularly at the rupture prone plaque shoulder region, where the shear stress on the vessel wall is highest ${ }^{81,82}$. Yet, direct evidence hereof is still lacking.

Taken together, it is likely to assume that functional aberrations of plaque microvasculature could indeed cause exacerbation of the atherosclerotic process. The human studies however show nothing more than guilt-by-association, and cannot confirm whether functional aberrations of plaque microvasculature could be causative. Experimental models of 
atherosclerosis however also provide ample evidence supporting an adverse role of plaque angiogenesis. Pro-angiogenic factors have been implicated in lesion formation ${ }^{54,83-86}$, while anti-angiogenic factors show the opposite effect, and reduce lesion formation ${ }^{74,87-92}$. One of the most elegant studies in this field by Moulton et al actually could show an increase in adventitial and intimal plaque vascularization in apolipoprotein-E (ApoE)/Collagen type XVIII (Col18) double-knockout mice, in concurrence with augmented plaque growth upon enhanced vascularization ${ }^{90}$. In general however, just few of these studies could show an actual causative effect on plaque vascularization, and angiogenesis-independent effects cannot be ruled out. Moreover, in small animal models, presence of plaque vascularization seems to be a rather infrequent phenomenon. Adventitial microvessels are seen frequently, and make up the majority of plaque vasculature studied. Yet, intraplaque vascularization seems to be a rare occurrence in mouse models, and only has been shown in a few models, e.g. the $p 22^{\text {phox }}$ model ${ }^{93}$, and some studies in apolipoprotein-E deficient $\left(\mathrm{ApoE}^{-/}\right)$and low density lipoprotein receptor deficient $\left(\mathrm{LDLr}^{-1-}\right)$ mice ${ }^{74,84,89,94}$. Of note, actual functionality of the described intraplaque vessels is debatable, since the intraplaque vessels at least in part appear to be invaginations derived from luminal endothelium ${ }^{74}$.

This addresses the important point of not only establishing the presence of plaque vascularization, but also gaining more insight into its localization and function in vivo, and establishing whether plaque vascularization in experimental models may be considered representative for human plaque vascularization. New imaging modalities, both microscopic and non-microscopic like magnetic resonance imaging (MRI), micro- or nanocomputed tomography (CT) ${ }^{95-97}$, will be of importance in studying this by examining whole (living) tissue.

The latter would also allow functional study of plaque vascularization, and could function as a platform for examining the effect of interventions. Assuming that plaque neovascularization indeed is negatively contributing to plaque development, two intervention strategies would come to mind: (i) antiangiogenic therapy or (ii) therapy focused on normalizing the leaky microvascular phenotype. Anti-angiogenic therapy would seem an attractive option, and showed a reduction in plaque burden in experimental models of atherosclerosis, but some important considerations should be made. One of the considerations is that anti-angiogenic therapy can, at this moment, not be 
applied locally. As such, sustained therapy as would be needed in atherosclerosis would induce overall inhibition of angiogenesis, also in natural processes like wound healing. More importantly, and as discussed previously, it cannot be excluded that plaque angiogenesis does exert some beneficial effects. These would include the before mentioned efflux of cholesterol, but also a decreased necrosis upon sustained plaque hypoxia.

Normalization of plaque microvessels would then be a sound alternative, as has been reviewed extensively over the last years $33,38,39,50,98,99$. The beneficial effects of plaque neovascularization, alleviating local hypoxia and potential cholesterol efflux, would still be accommodated, yet without the leakage of erythrocytes, lipids and inflammatory cells into the plaque. This concept has received a lot of attention in other diseases involving pathologic angiogenesis, like tumorigenesis or certain hereditary disorders, and could consist of restoring endothelial barrier function or pericyte coverage ${ }^{39,100}$. A key example proving the potential of vessel normalization is a study by Lebrin et al showing that pharmacological intervention using thalidomide stimulated vessel maturation in patients with hereditary hemorrhagic telangiectasia, mostly by increasing pericyte coverage ${ }^{100}$. Recently, more targeted approaches of vessel normalization, like blocking of PIGF, neutralization of Ang-2, or inhibiting the angiogenic $\alpha_{v} \beta_{3}$ integrin activity are proving relatively successful in cancer treatment, and may be potential future targets for microvessel normalization in atherosclerosis ${ }^{38,39,50,98}$. 


\section{Atherosclerosis and lymphangiogenesis}

In contrast to the early discovery of vasa vasorum expansion, the possible involvement of the lymphatic system in atherosclerosis has only very recently gained more interest. This is quite surprising, taking into account that the lymphatic system already has been implicated in regulation of lipid efflux and inflammation decades ago.

\section{General background}

The lymphatic system for some time had been an underappreciated system with regard to its function. Initially, it was regarded as a simple return system for fluid buildup in tissues due to fluid loss via the arterial system caused by pressure and osmotic gradients. The current view is more refined, suggesting an important role in regulation of lipid transport, especially in the intestine and regulation of inflammation ${ }^{101-103}$.

Lymph vessels are more irregularly shaped vessels, which are characterized by interendothelial valve-like openings ${ }^{104-107}$. Additionally, all lymph vessels with the exception of the lymph capillaries contain intraluminal valves to prevent backflow of lymph fluid. Lymph vessels, and in conjunction lymph nodes, have been implicated in edema formation, cancer, asthma, and several inflammatory diseases ${ }^{108-110}$.

Lymphangiogenesis is mostly present during embryogenesis, and its molecular regulation has been described elegantly by Adams and Alitalo ${ }^{30}$. During embryogenesis, the first lymphatic endothelial cells (LECS) are derived from the embryonic cardinal vein. A few ECs of the cardinal vein induce expression of the transcription factor prospero-related homeobox-1 (Prox-1) and migrate away from the cardinal vein. Under the influence of additional factors like the tyrosine kinase SYK, the LECs phenotypically completely separate from blood vessels and form the so-called lymphatic sac. In adults, Prox-1 is also a required factor for induction of lymphangiogenesis. Following formation of the lymphatic sac, sprouting and formation of the first lymphatic plexus is induced by VEGF-C and VEGFR-3 (Figure 3), in combination with the guidance receptor neuropilin-2 (NRP-2) ${ }^{111,112}$. After formation of the lymphatic network, the lymph vessels undergo a maturation step, including valve formation, under influence of Ang-2, podoplanin, ephrin-B2 and forkhead box protein C2 (Fox-C2). 


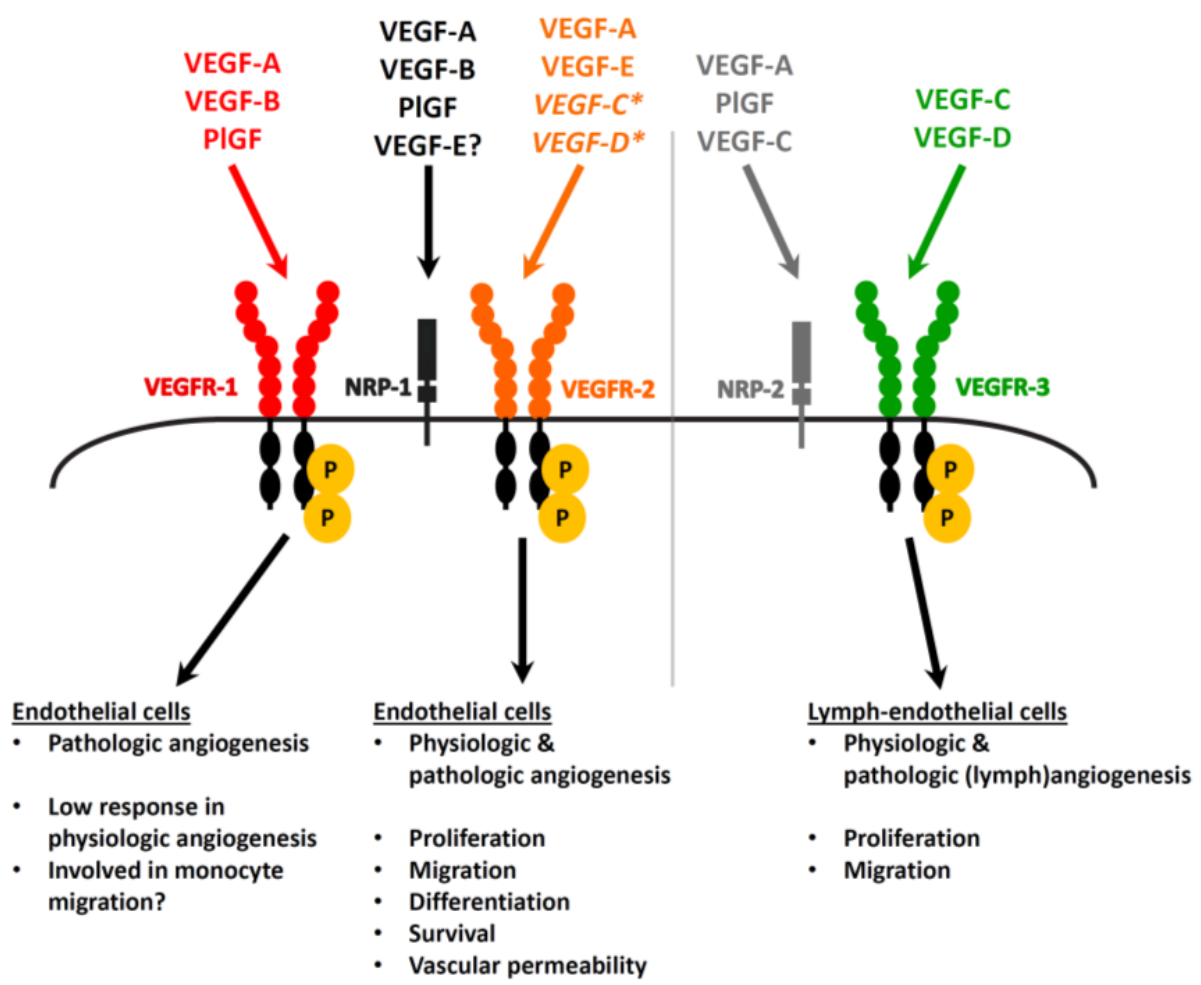

Figure 3. Both angiogenesis and lymphangiogenesis are regulated by the VEGF and VEGFRfamily. In angiogenesis, VEGFR-1 and predominantly VEGFR-2 on the endothelial cells is stimulated by PIGF and VEGF-A, $-B$ and $-E$, and facilitate processes involved in angiogenesis, e.g. proliferation and migration. Homologously, lymphatic endothelial cells express VEGFR-3, which upon activation by VEGF-C and VEGF-D elicits a lymphangiogenic response, but have also been described to elicit an angiogenic response (asterisks). Accessory neuropilin (NRP) receptors are required to potentiate the (lymph)angiogenic response and are additionally involved as guidance molecules. Figure based on ${ }^{50,113}$.

Lymphangiogenesis in adults follows the same principle and has been studied mainly in oncology and inflammatory bowel disease. There is a great homology between the pathways involved in lymphangiogenesis and angiogenesis, and the processes do share a similar pattern ${ }^{30,102,113 .}$ Additionally, recent reports also indicate towards a VEGF-independent lymphangiogenesis, i.e. mechano-induced lymphangiogenesis (dependent on integrin activation of VEGFR-3), suggesting a mechanism which responds directly to pressure buildup (Figure 4) ${ }^{114}$. Next to Prox-1, podoplanin and VEGFR-3, lymphatic vessel endothelial hyaluronan receptor (LYVE-1), present 
on lymphatic capillaries, is regarded as one of the key markers for lymph vessels.

Evidence suggests that lymphangiogenesis under pathologic conditions occurs similarly to its physiological equivalent, although the mechanisms do differ slightly between intranodal and extranodal lymphangiogenesis ${ }^{102}$. Intranodal lymphangiogenesis is characterized by a response to increased lymph flow and antigen presentation, while extranodal lymphangiogenesis is responsive to inflammatory stimuli and local fluid buildup ${ }^{102}$.

\section{Lymphangiogenesis in atherosclerosis}

The lymphatic system was first described in human arteries by Johnson in the late $1960 \mathrm{~s}^{115}$. Its function was regarded to be fluid resorption from the extravascular tissue. With time, increasing evidence gathered that the lymphatic system could also be involved in lipid transport and inflammation, in the adventitia of healthy larger blood vessels ${ }^{116}$, as well as under pathologic conditions like atherosclerosis ${ }^{21,117-121}$.

The first record of lymph vessels in atherosclerosis dates back to 1970, where lymphatics were studied in a rat model of atherosclerosis, where it was linked to increased edema formation ${ }^{118}$. Around the 1980s, Sims described increases in atherosclerotic disease in patients with obstructed lymphatics due to carcinomas, as well as an increase in edema formation in patients suffering from coronary atherosclerosis ${ }^{122,123}$. Around the same period, Wollinsky suggested that upon a dysbalance in the influx and efflux of lipids and inflammatory cells, as is the case in atherosclerosis, the lymphatic system might be implicated ${ }^{124}$. It took until the early 1990s before Miller et al suggested a potential causative role for lymphatics and lymphangiogenesis in (coronary) atherosclerosis ${ }^{119}$. Nakano et al described the presence of lymphangiogenic factors like VEGF-C and -D in human plaques in the coronary artery ${ }^{52}$, and a study from 2011 by Wong et al confirmed these findings ${ }^{125}$. Just recently, the first study was published which actually showed lymphatic (micro)vasculature in human atherosclerotic lesions using specific markers for lymph vessels ${ }^{27}$. Syväranta et al and Drozdz et al furthermore showed a positive correlation between the number of lymph vessels and the severity of atherosclerosis, comparable to microvessel expansion ${ }^{21,120}$. 

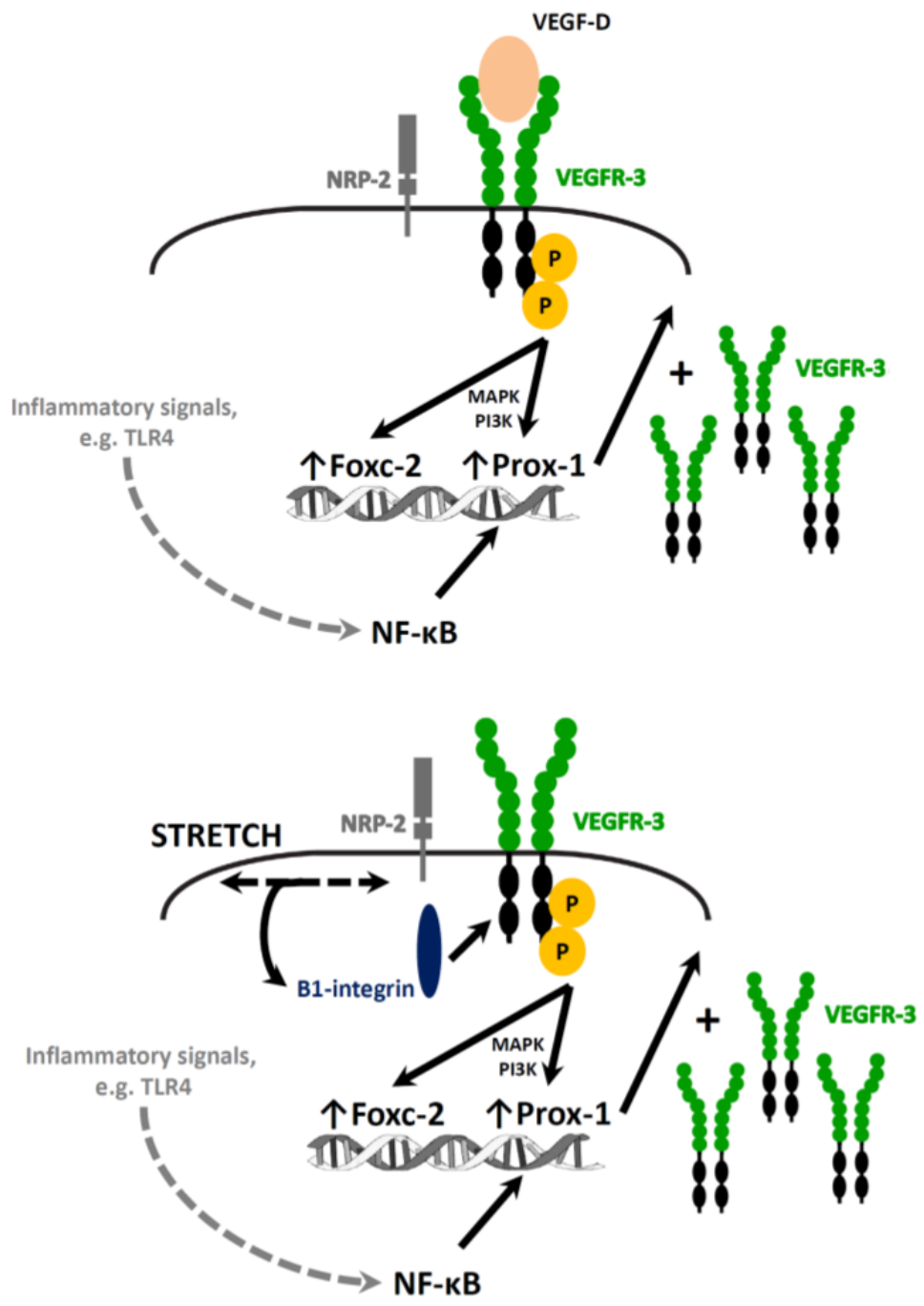

Figure 4. Lymphangiogenesis can be induced classically and non-classically. Lymphangiogenesis is classically induced by activation of VEGFR-3 by VEGF-C and VEGF-D (A). Binding of the ligands to their receptor phosphorylates the intracellular domains, subsequently inducing an upregulation of e.g. Fox-C2 and Prox-1 expression via phosphoinositide 3-kinase (PI3K) and mitogen-activated protein kinase (MAPK). Nuclear factor $K B$ (NF-KB) signaling can enhance this response. Alternatively, lymphangiogenesis can be induced independent of VEGFR-3 activation by VEGF-C and VEGF-D. Recently, a stretch-induced mechanism of lymphangiogenesis has been described ${ }^{114}$. Stretch of lymphatic endothelial cells by e.g. edema formation will activate 81 integrins, allowing direct phosphorylation of the intracellular domain of VEGFR-3 (B). Both mechanisms increase expression of VEGFR-3 on the lymphendothelial cell surface, and induce proliferation and migration of lymphatic endothelial cells. 


\section{CHAPTER 1}

Besides the histological evidence presented above, other evidence also supported a contribution of lymphangiogenesis to atherosclerosis. The involvement of draining lymph nodes of atherosclerotic arteries, and thus conceivably the lymph vessels facilitating transport of inflammatory cells like $T$ cells and dendritic cells (DCs) away from the plaque towards draining lymph nodes, has been described ${ }^{101}, 126,127$. Several studies have even described formation of tertiary lymphoid organs (TLOs) or vascular associated lymphoid tissue (VALT) in the adventitia of atherosclerotic arteries, in which an abundance of microvessels and lymphatic capillaries is present ${ }^{128,}{ }^{129}$. In addition, it is known that lymphangiogenesis is stimulated by inflammation, as a result of expression of lymphangiogenic molecules by macrophages, B cells and $T$ cells ${ }^{102,130,131}$. Of note, inflammatory cells are also able to express antilymphangiogenic molecules like transforming growth factor- $\beta$ (TGF- $\beta$ ) and interferon- $\gamma(\text { IFN- }-)^{130,132}$.

However, there is still a lot to clarify on the interactions of the different types of inflammatory cells and the lymphatic vessels. Moreover, also the role of lymph vessels in (experimental) atherosclerosis is to a large extent unresolved, as reflected by the absence of papers published on this subject, and will require more study. An improved understanding of the role of lymphangiogenesis in disease will potentially lead to new targeted therapies. 


\section{Hypothesis and outline of this thesis}

It is clear that both angiogenesis and lymphangiogenesis play a role in atherosclerotic plaque progression, although their contributions are incompletely understood. The hypothesis of this thesis is that both angiogenesis and lymphangiogenesis are of key importance to atherosclerosis, and that the aberrant function of blood vessels and lymph vessels under inflammatory conditions promote atherosclerosis progression. To investigate the role of angiogenesis and lymphangiogenesis in atherosclerosis, we performed several studies in both human atherosclerosis and experimental models of atherosclerosis.

Our first approach was focused on establishing functional imaging of atherosclerotic plaque vascularization. To this end, we used an in vivo multiphoton laser scanning microscopy (MPLSM) approach. Chapter 2 reviews the current state of in vivo cardiovascular imaging using MPLSM, and discusses the benefits and shortcomings of the technique. In chapter 3, we applied this technique to study plaque neovascularization in a murine model for atherosclerosis ( $\mathrm{ApoE}^{-1-}$ mice). We could establish not only the occurrence of adventitial plaque neovascularization in these murine models, but also the dysfunctional, leakier phenotype of plaque-associated vasa vasorum.

In order to further explore possible mediators of this leaky phenotype, we investigated the function of two important endothelial junction molecules, JAM-A and VE-cadherin. In chapter 4, we focused on the role of the adhesion molecule JAM-A on endothelial permeability and leukocyte adhesion. It was shown that in the large arteries, JAM-A was critical for leukocyte adhesion and transmigration, and that distortion of physiological endothelial JAM-A distribution induced increased leukocyte recruitment. Another endothelial junction molecule involved in endothelial barrier function, VE-cadherin, was studied in chapter $\mathbf{5}$. Here, we studied the effect of acute deterioration of the endothelial barrier function in plaque microvessels. We could show that specific blocking of VE-cadherin could induce increased microvascular permeability in control microvessels, but not in plaque microvessels, suggesting that either VE-cadherin may already be dysfunctional in plaqueassociated vasa vasorum or alternatively is not involved in microvascular permeability in plaque-associated microvessels at all. 


\section{CHAPTER 1}

Next, we went from studies in murine models to a genomics-based study in human atherosclerotic lesions samples, to identify novel targets involved in plaque angiogenesis and lymphangiogenesis. In chapter 6, we correlated microarray data from stable and ruptured atherosclerotic lesions to (lymphatic) vascularization state, and we were able to establish a set of genes and five miRNAs which may be involved in regulation of angiogenesis in atherosclerosis. For regulation of lymphangiogenesis in atherosclerosis, we found a low correlation between the detected genes and the lymphatic marker D2-40, and thus will require more detailed study in the future.

In order to gain a better understanding of the role of the lymphatic system in atherosclerosis, we performed several intervention studies in atherosclerotic $\mathrm{ApoE}^{-/-}$mice. In chapter 7, it was shown that removal of the lymph node draining the carotid artery, as well as after inhibition of lymphangiogenesis, had an adverse effect on plaque progression, characterized by an increased plaque size and a more inflammatory phenotype with significantly increased $\mathrm{T}$ cell numbers. This enhanced inflammatory phenotype was also observed after inhibition of lymphangiogenesis, suggesting a beneficial role of the lymphatic system during plaque progression.

Lastly, in chapter $\mathbf{8}$, the results described in this thesis and potential implications hereof were discussed and put in perspective. Also, future directions were given for further study of angiogenesis and lymphangiogenesis in atherosclerosis. 


\section{References}

1. Lloyd-Jones D, Adams R, Carnethon $\mathrm{M}$, et al. Heart disease and stroke statistics--2009 update: A report from the american heart association statistics committee and stroke statistics subcommittee. Circulation. 2009;119:e21-181

2. Mukherjee D, Patil CG. Epidemiology and the global burden of stroke. World Neurosurg. 2011;76:S85-90

3. Perk J, De Backer G, Gohlke H, et al. European guidelines on cardiovascular disease prevention in clinical practice (version 2012): The fifth joint task force of the european society of cardiology and other societies on cardiovascular disease prevention in clinical practice (constituted by representatives of nine societies and by invited experts). Atherosclerosis. 2012;223:1-68

4. Sanz J, Moreno PR, Fuster V. The year in atherothrombosis. J Am Coll Cardiol. 2012;60:932-942

5. Mathers CD, Loncar D. Projections of global mortality and burden of disease from 2002 to 2030. PLoS Med. 2006;3:e442

6. Roger VL, Go AS, Lloyd-Jones DM, et al. Heart disease and stroke statistics-2011 update: A report from the american heart association. Circulation. 2011;123:e18-e209

7. Virmani R, Kolodgie FD, Burke AP, et al. Lessons from sudden coronary death: A comprehensive morphological classification scheme for atherosclerotic lesions. Arterioscler Thromb Vasc Biol. 2000;20:1262-1275

8. Hansson GK. Inflammation, atherosclerosis, and coronary artery disease. $N$ Engl J Med. 2005;352:1685-1695

9. Libby P. Atherosclerosis: The new view. Sci Am. 2002;286:46-55

10. Lusis AJ. Atherosclerosis. Nature. 2000;407:233-241

11. Zernecke A, Weber $C$. Chemokines in the vascular inflammatory response of atherosclerosis. Cardiovasc Res. 2010;86:192-201

12. Hansson GK, Hermansson A. The immune system in atherosclerosis. Nat Immunol. 2011;12:204-212

13. Libby P. Inflammation in atherosclerosis. Arterioscler Thromb Vasc Biol. 2012;32:2045-2051

14. Soehnlein O, Lindbom L, Weber C. Mechanisms underlying neutrophilmediated monocyte recruitment. Blood. 2009;114:4613-4623

15. Weber C, Zernecke A, Libby P. The multifaceted contributions of leukocyte subsets to atherosclerosis: Lessons from mouse models. Nat Rev Immunol. 2008;8:802-815

16. Businaro R, Tagliani A, Buttari B, et al. Cellular and molecular players in the atherosclerotic plaque progression. Ann N Y Acad Sci. 2012;1262:134-141

17. Schrijvers DM, De Meyer GR, Martinet W. Autophagy in atherosclerosis: A potential drug target for plaque stabilization. Arterioscler Thromb Vasc Biol. 2011;31:2787-2791 
18. Thorp E, Subramanian M, Tabas I. The role of macrophages and dendritic cells in the clearance of apoptotic cells in advanced atherosclerosis. Eur J Immunol. 2011;41:2515-2518

19. Sanz J, Fayad ZA. Imaging of atherosclerotic cardiovascular disease. Nature. 2008;451:953-957

20. Ketelhuth DF, Back $M$. The role of matrix metalloproteinases in atherothrombosis. Curr Atheroscler Rep. 2011;13:162-169

21. Drozdz K, Janczak D, Dziegiel $\mathrm{P}$, et al. Adventitial lymphatics and atherosclerosis. Lymphology. 2012;45:26-33

22. Moreno PR, Purushothaman $M$, Purushothaman KR. Plaque neovascularization: Defense mechanisms, betrayal, or a war in progress. Ann N Y Acad Sci. 2012;1254:7-17

23. Naghavi $M$, Libby $P$, Falk $E$, et al. From vulnerable plaque to vulnerable patient: A call for new definitions and risk assessment strategies: Part i. Circulation. 2003;108:1664-1672

24. Sluimer JC, Gasc JM, van Wanroij JL, et al. Hypoxia, hypoxia-inducible transcription factor, and macrophages in human atherosclerotic plaques are correlated with intraplaque angiogenesis. J Am Coll Cardiol. 2008;51:12581265

25. Moreno PR, Purushothaman KR, Fuster V, et al. Plaque neovascularization is increased in ruptured atherosclerotic lesions of human aorta: Implications for plaque vulnerability. Circulation. 2004;110:2032-2038

26. Sluimer JC, Daemen MJ. Novel concepts in atherogenesis: Angiogenesis and hypoxia in atherosclerosis. J Pathol. 2009;218:7-29

27. Kholova I, Dragneva G, Cermakova $P$, et al. Lymphatic vasculature is increased in heart valves, ischaemic and inflamed hearts and in cholesterol-rich and calcified atherosclerotic lesions. Eur J Clin Invest. 2011;41:487-497

28. Köster W. Endarteritis and arteritis. Berl Klin Wochenschr. 1876;13:343-345

29. Wolinsky H, Glagov S. Nature of species differences in the medial distribution of aortic vasa vasorum in mammals. Circ Res. 1967;20:409-421

30. Adams RH, Alitalo K. Molecular regulation of angiogenesis and lymphangiogenesis. Nat Rev Mol Cell Biol. 2007;8:464-478

31. Carmeliet P. Blood vessels and nerves: Common signals, pathways and diseases. Nat Rev Genet. 2003;4:710-720

32. Carmeliet P. Angiogenesis in health and disease. Nat Med. 2003;9:653-660

33. Potente $M$, Gerhardt $H$, Carmeliet $P$. Basic and therapeutic aspects of angiogenesis. Cell. 2011;146:873-887

34. Carmeliet P, Jain RK. Molecular mechanisms and clinical applications of angiogenesis. Nature. 2011;473:298-307

35. Eilken HM, Adams RH. Dynamics of endothelial cell behavior in sprouting angiogenesis. Curr Opin Cell Biol. 2010;22:617-625

36. Iruela-Arispe ML, Davis GE. Cellular and molecular mechanisms of vascular lumen formation. Dev Cell. 2009;16:222-231

37. Naik MU, Stalker TJ, Brass LF, et al. Jam-a protects from thrombosis by suppressing integrin alphaiibbeta3-dependent outside-in signaling in platelets. Blood. 2012;119:3352-3360 
38. Carmeliet P, Jain RK. Principles and mechanisms of vessel normalization for cancer and other angiogenic diseases. Nat Rev Drug Discov. 2011;10:417-427

39. Goel S, Duda DG, Xu L, et al. Normalization of the vasculature for treatment of cancer and other diseases. Physiol Rev. 2011;91:1071-1121

40. Paterson JC. Vascularization and hemorrhage of the intima of arteriosclerotic coronary arteries. Arch Path. 1936;22:313-324

41. Grundy SM. Atherosclerosis: Pathology, pathogenesis, and role of risk factors. Dis Mon. 1983;29:1-58

42. Schwartz SM. Cellular proliferation in atherosclerosis and hypertension. Proc Soc Exp Biol Med. 1983;173:1-13

43. Kamat BR, Galli SJ, Barger AC, et al. Neovascularization and coronary atherosclerotic plaque: Cinematographic localization and quantitative histologic analysis. Hum Pathol. 1987;18:1036-1042

44. Virmani R, Kolodgie FD, Burke AP, et al. Atherosclerotic plaque progression and vulnerability to rupture: Angiogenesis as a source of intraplaque hemorrhage. Arterioscler Thromb Vasc Biol. 2005;25:2054-2061

45. Jeziorska M, Woolley DE. Neovascularization in early atherosclerotic lesions of human carotid arteries: Its potential contribution to plaque development. Hum Pathol. 1999;30:919-925

46. Cliff WJ, Heathcote CR, Moss NS, et al. The coronary arteries in cases of cardiac and noncardiac sudden death. Am J Pathol. 1988;132:319-329

47. McCarthy MJ, Loftus IM, Thompson MM, et al. Angiogenesis and the atherosclerotic carotid plaque: An association between symptomatology and plaque morphology. J Vasc Surg. 1999;30:261-268

48. Mofidi R, Crotty TB, McCarthy $\mathrm{P}$, et al. Association between plaque instability, angiogenesis and symptomatic carotid occlusive disease. $\mathrm{Br} J$ Surg. 2001;88:945-950

49. Alpern-Elran $\mathrm{H}$, Morog N, Robert $\mathrm{F}$, et al. Angiogenic activity of the atherosclerotic carotid artery plaque. J Neurosurg. 1989;70:942-945

50. Vuorio T, Jauhiainen S, Yla-Herttuala S. Pro- and anti-angiogenic therapy and atherosclerosis with special emphasis on vascular endothelial growth factors.

Expert Opin Biol Ther. 2012;12:79-92

51. Chen YX, Nakashima Y, Tanaka K, et al. Immunohistochemical expression of vascular endothelial growth factor/vascular permeability factor in atherosclerotic intimas of human coronary arteries. Arterioscler Thromb Vasc Biol. 1999;19:131-139

52. Nakano T, Nakashima $\mathrm{Y}$, Yonemitsu $\mathrm{Y}$, et al. Angiogenesis and lymphangiogenesis and expression of lymphangiogenic factors in the atherosclerotic intima of human coronary arteries. Hum Pathol. 2005;36:330340

53. Ignatescu MC, Gharehbaghi-Schnell E, Hassan A, et al. Expression of the angiogenic protein, platelet-derived endothelial cell growth factor, in coronary atherosclerotic plaques: In vivo correlation of lesional microvessel density and constrictive vascular remodeling. Arterioscler Thromb Vasc Biol. 1999;19:2340-2347 
54. Pilarczyk K, Sattler KJ, Galili O, et al. Placenta growth factor expression in human atherosclerotic carotid plaques is related to plaque destabilization. Atherosclerosis. 2008;196:333-340

55. Post S, Peeters W, Busser E, et al. Balance between angiopoietin-1 and angiopoietin-2 is in favor of angiopoietin-2 in atherosclerotic plaques with high microvessel density. J Vasc Res. 2008;45:244-250

56. Nikkari ST, O'Brien KD, Ferguson $\mathrm{M}$, et al. Interstitial collagenase (mmp-1) expression in human carotid atherosclerosis. Circulation. 1995;92:1393-1398

57. Pasterkamp G, Schoneveld AH, Hijnen DJ, et al. Atherosclerotic arterial remodeling and the localization of macrophages and matrix metalloproteases 1, 2 and 9 in the human coronary artery. Atherosclerosis. 2000;150:245-253

58. Bot I, Biessen EA. Mast cells in atherosclerosis. Thromb Haemost. 2011;106:820-826

59. Kaartinen M, Penttila A, Kovanen PT. Mast cells accompany microvessels in human coronary atheromas: Implications for intimal neovascularization and hemorrhage. Atherosclerosis. 1996;123:123-131

60. Lappalainen $\mathrm{H}$, Laine $\mathrm{P}$, Pentikainen $\mathrm{MO}$, et al. Mast cells in neovascularized human coronary plaques store and secrete basic fibroblast growth factor, a potent angiogenic mediator. Arterioscler Thromb Vasc Biol. 2004;24:18801885

61. Hao NB, Lu MH, Fan YH, et al. Macrophages in tumor microenvironments and the progression of tumors. Clin Dev Immunol. 2012;2012:948098

62. Mapp PI, Walsh DA. Mechanisms and targets of angiogenesis and nerve growth in osteoarthritis. Nat Rev Rheumatol. 2012;8:390-398

63. Torres Filho IP, Leunig M, Yuan F, et al. Noninvasive measurement of microvascular and interstitial oxygen profiles in a human tumor in scid mice. Proc Natl Acad Sci U S A. 1994;91:2081-2085

64. Moreno PR, Purushothaman KR, Sirol M, et al. Neovascularization in human atherosclerosis. Circulation. 2006;113:2245-2252

65. Nicholls SJ, Borgman M, Nissen SE, et al. Impact of statins on progression of atherosclerosis: Rationale and design of saturn (study of coronary atheroma by intravascular ultrasound: Effect of rosuvastatin versus atorvastatin). Curr Med Res Opin. 2011;27:1119-1129

66. Barker SG, Talbert A, Cottam S, et al. Arterial intimal hyperplasia after occlusion of the adventitial vasa vasorum in the pig. Arterioscler Thromb. 1993;13:70-77

67. Booth RF, Martin JF, Honey AC, et al. Rapid development of atherosclerotic lesions in the rabbit carotid artery induced by perivascular manipulation. Atherosclerosis. 1989;76:257-268

68. Nakata Y, Kamiya K. An experimental study on the vascular lesions caused by obstruction of the vaso vasorum. li. Special consideration on the deposition of fat into vascular wall. Jpn Circ J. 1970;34:1029-1034

69. Nakata Y, Shionoya S. Vascular lesions due to obstruction of the vasa vasorum. Nature. 1966;212:1258-1259

70. Nakata Y, Shionoya S, Matsubara J, et al. An experimental study on the vascular lesions caused by disturbance of the vasa vasorum. 3. Influence of 
obstruction of the venous side of the vasa vasorum and the periaortic vein. Jpn Circ J. 1972;36:945-951

71. de Boer OJ, van der Wal AC, Teeling $P$, et al. Leucocyte recruitment in rupture prone regions of lipid-rich plaques: A prominent role for neovascularization? Cardiovasc Res. 1999;41:443-449

72. Doyle B, Caplice N. Plaque neovascularization and antiangiogenic therapy for atherosclerosis. J Am Coll Cardiol. 2007;49:2073-2080

73. Ribatti D, Levi-Schaffer F, Kovanen PT. Inflammatory angiogenesis in atherogenesis--a double-edged sword. Ann Med. 2008;40:606-621

74. Hauer $A D$, van Puijvelde $G H$, Peterse $N$, et al. Vaccination against vegfr2 attenuates initiation and progression of atherosclerosis. Arterioscler Thromb Vasc Biol. 2007;27:2050-2057

75. Kumamoto $M$, Nakashima $Y$, Sueishi K. Intimal neovascularization in human coronary atherosclerosis: Its origin and pathophysiological significance. Hum Pathol. 1995;26:450-456

76. Depre C, Havaux X, Wijns W. Neovascularization in human coronary atherosclerotic lesions. Cathet Cardiovasc Diagn. 1996;39:215-220

77. Sluimer JC, Kolodgie FD, Bijnens AP, et al. Thin-walled microvessels in human coronary atherosclerotic plaques show incomplete endothelial junctions relevance of compromised structural integrity for intraplaque microvascular leakage. J Am Coll Cardiol. 2009;53:1517-1527

78. de Boer OJ, van der Wal AC, Teeling $P$, et al. Leucocyte recruitment in rupture prone regions of lipid-rich plaques: A prominent role for neovascularization? Cardiovasc Res. 1999;41:443-449

79. Jeziorska M, Woolley DE. Local neovascularization and cellular composition within vulnerable regions of atherosclerotic plaques of human carotid arteries. J Pathol. 1999;188:189-196

80. Moos MP, John N, Grabner R, et al. The lamina adventitia is the major site of immune cell accumulation in standard chow-fed apolipoprotein e-deficient mice. Arterioscler Thromb Vasc Biol. 2005;25:2386-2391

81. Gijsen FJ, Wentzel JJ, Thury A, et al. Strain distribution over plaques in human coronary arteries relates to shear stress. Am J Physiol Heart Circ Physiol. 2008;295:H1608-1614

82. Jeziorska M, Woolley DE. Local neovascularization and cellular composition within vulnerable regions of atherosclerotic plaques of human carotid arteries. J Pathol. 1999;188:189-196

83. Celletti FL, Waugh JM, Amabile PG, et al. Vascular endothelial growth factor enhances atherosclerotic plaque progression. Nat Med. 2001;7:425-429

84. Lucerna $M$, Zernecke A, de Nooijer $\mathrm{R}$, et al. Vascular endothelial growth factor-a induces plaque expansion in apoe knock-out mice by promoting de novo leukocyte recruitment. Blood. 2007;109:122-129

85. Luttun A, Tjwa M, Carmeliet P. Placental growth factor (plgf) and its receptor flt-1 (vegfr-1): Novel therapeutic targets for angiogenic disorders. Ann $N Y$ Acad Sci. 2002;979:80-93 
86. Tanaka K, Nagata D, Hirata $Y$, et al. Augmented angiogenesis in adventitia promotes growth of atherosclerotic plaque in apolipoprotein e-deficient mice. Atherosclerosis. 2011;215:366-373

87. Kolodgie FD, Narula J, Yuan C, et al. Elimination of neoangiogenesis for plaque stabilization: Is there a role for local drug therapy? J Am Coll Cardiol. 2007;49:2093-2101

88. Luttun A, Brusselmans $\mathrm{K}$, Fukao $\mathrm{H}$, et al. Loss of placental growth factor protects mice against vascular permeability in pathological conditions. Biochem Biophys Res Commun. 2002;295:428-434

89. Moulton KS, Heller E, Konerding MA, et al. Angiogenesis inhibitors endostatin or tnp-470 reduce intimal neovascularization and plaque growth in apolipoprotein e-deficient mice. Circulation. 1999;99:1726-1732

90. Moulton KS, Olsen BR, Sonn S, et al. Loss of collagen xviii enhances neovascularization and vascular permeability in atherosclerosis. Circulation. 2004;110:1330-1336

91. Moulton KS, Vakili K, Zurakowski D, et al. Inhibition of plaque neovascularization reduces macrophage accumulation and progression of advanced atherosclerosis. Proc Natl Acad Sci U S A. 2003;100:4736-4741

92. Petrovan RJ, Kaplan CD, Reisfeld RA, et al. DNA vaccination against vegf receptor 2 reduces atherosclerosis in Idl receptor-deficient mice. Arterioscler Thromb Vasc Biol. 2007;27:1095-1100

93. Khatri JJ, Johnson C, Magid R, et al. Vascular oxidant stress enhances progression and angiogenesis of experimental atheroma. Circulation. 2004;109:520-525

94. de Nooijer R, Verkleij CJ, von der Thusen JH, et al. Lesional overexpression of matrix metalloproteinase-9 promotes intraplaque hemorrhage in advanced lesions but not at earlier stages of atherogenesis. Arterioscler Thromb Vasc Biol. 2006;26:340-346

95. Calcagno C, Cornily JC, Hyafil F, et al. Detection of neovessels in atherosclerotic plaques of rabbits using dynamic contrast enhanced mri and 18f-fdg pet. Arterioscler Thromb Vasc Biol. 2008;28:1311-1317

96. Winter PM, Morawski AM, Caruthers SD, et al. Molecular imaging of angiogenesis in early-stage atherosclerosis with alpha(v)beta3-integrintargeted nanoparticles. Circulation. 2003;108:2270-2274

97. Langheinrich AC, Michniewicz A, Sedding DG, et al. Correlation of vasa vasorum neovascularization and plaque progression in aortas of apolipoprotein e(-/-)/low-density lipoprotein(-/-) double knockout mice. Arterioscler Thromb Vasc Biol. 2006;26:347-352

98. De Bock K, Cauwenberghs S, Carmeliet P. Vessel abnormalization: Another hallmark of cancer? Molecular mechanisms and therapeutic implications. Curr Opin Genet Dev. 2011;21:73-79

99. Yla-Herttuala S, Bentzon JF, Daemen $\mathrm{M}$, et al. Stabilisation of atherosclerotic plaques. Position paper of the european society of cardiology (esc) working group on atherosclerosis and vascular biology. Thromb Haemost. 2011;106:119 
100. Lebrin F, Srun S, Raymond K, et al. Thalidomide stimulates vessel maturation and reduces epistaxis in individuals with hereditary hemorrhagic telangiectasia. Nat Med. 2010;16:420-428

101. Forster R, Braun A, Worbs T. Lymph node homing of t cells and dendritic cells via afferent lymphatics. Trends Immunol. 2012;33:271-280

102. Kim H, Kataru RP, Koh GY. Regulation and implications of inflammatory lymphangiogenesis. Trends Immunol. 2012;33:350-356

103. Rovenska E, Rovensky J. Lymphatic vessels: Structure and function. Isr Med Assoc J. 2011;13:762-768

104. Casley-Smith JR. The fine structure and functioning of tissue channels and lymphatics. Lymphology. 1980;13:177-183

105. Leak LV. Electron microscopic observations on lymphatic capillaries and the structural components of the connective tissue-lymph interface. Microvasc Res. 1970;2:361-391

106. Leak LV, Burke JF. Fine structure of the lymphatic capillary and the adjoining connective tissue area. Am J Anat. 1966;118:785-809

107. Oh SJ, Jeltsch MM, Birkenhager R, et al. Vegf and vegf-c: Specific induction of angiogenesis and lymphangiogenesis in the differentiated avian chorioallantoic membrane. Dev Biol. 1997;188:96-109

108. Alitalo K, Tammela T, Petrova TV. Lymphangiogenesis in development and human disease. Nature. 2005;438:946-953

109. Nagahashi M, Ramachandran S, Rashid OM, et al. Lymphangiogenesis: A new player in cancer progression. World J Gastroenterol. 2010;16:4003-4012

110. Flister MJ, Wilber A, Hall KL, et al. Inflammation induces lymphangiogenesis through up-regulation of vegfr-3 mediated by nf-kappab and prox1. Blood. 2010;115:418-429

111. Saharinen P, Petrova TV. Molecular regulation of lymphangiogenesis. Ann N Y Acad Sci. 2004;1014:76-87

112. Takahashi H, Shibuya M. The vascular endothelial growth factor (vegf)/vegf receptor system and its role under physiological and pathological conditions. Clin Sci (Lond). 2005;109:227-241

113. Lohela M, Saaristo A, Veikkola $T$, et al. Lymphangiogenic growth factors, receptors and therapies. Thromb Haemost. 2003;90:167-184

114. Planas-Paz L, Strilic B, Goedecke A, et al. Mechanoinduction of lymph vessel expansion. Embo J. 2012;31:788-804

115. Johnson RA. Lymphatics of blood vessels. Lymphology. 1969;2:44-56

116. Sacchi G, Weber E, Comparini L. Histological framework of lymphatic vasa vasorum of major arteries: An experimental study. Lymphology. 1990;23:135139

117. Alitalo K, Carmeliet P. Molecular mechanisms of lymphangiogenesis in health and disease. Cancer Cell. 2002;1:219-227

118. Jellinek $H$, Veress $B$, Balint $A$, et al. Lymph vessels of rat aorta and their changes in experimental atherosclerosis: An electron microscopic study. Exp Mol Pathol. 1970;13:370-376

119. Miller AJ, DeBoer A, Palmer A. The role of the lymphatic system in coronary atherosclerosis. Med Hypotheses. 1992;37:31-36 
120. Syvaranta S, Helske S, Lappalainen J, et al. Lymphangiogenesis in aortic valve stenosis--novel regulatory roles for valvular myofibroblasts and mast cells. Atherosclerosis. 2012;221:366-374

121. Xu X, Lin $\mathrm{H}, \mathrm{Lv} \mathrm{H}$, et al. Adventitial lymphatic vessels -- an important role in atherosclerosis. Med Hypotheses. 2007;69:1238-1241

122. Sims FH. The arterial wall in malignant disease. Atherosclerosis. 1979;32:445450

123. Sims FH. A comparison of coronary and internal mammary arteries and implications of the results in the etiology of arteriosclerosis. Am Heart J. 1983;105:560-566

124. Wolinsky H. A proposal linking clearance of circulating lipoproteins to tissue metabolic activity as a basis for understanding atherogenesis. Circ Res. 1980;47:301-311

125. Wong BW, Wong $\mathrm{D}$, Luo $\mathrm{H}$, et al. Vascular endothelial growth factor-d is overexpressed in human cardiac allograft vasculopathy and diabetic atherosclerosis and induces endothelial permeability to low-density lipoproteins in vitro. J Heart Lung Transplant. 2011;30:955-962

126. Packard RR, Shi GP. Atherosclerosis progression and monocyte emigration from plaque. Future Cardiol. 2006;2:415-418

127. Randolph GJ. Emigration of monocyte-derived cells to lymph nodes during resolution of inflammation and its failure in atherosclerosis. Curr Opin Lipidol. 2008;19:462-468

128. Grabner R, Lotzer K, Dopping S, et al. Lymphotoxin beta receptor signaling promotes tertiary lymphoid organogenesis in the aorta adventitia of aged apoe-/- mice. J Exp Med. 2009;206:233-248

129. Weih F, Grabner R, Hu D, et al. Control of dichotomic innate and adaptive immune responses by artery tertiary lymphoid organs in atherosclerosis. Front Physiol. 2012;3:226

130. Zampell JC, Avraham T, Yoder N, et al. Lymphatic function is regulated by a coordinated expression of lymphangiogenic and anti-lymphangiogenic cytokines. Am J Physiol Cell Physiol. 2012;302:C392-404

131. Kim KE, Koh YJ, Jeon $\mathrm{BH}$, et al. Role of $\mathrm{cd} 11 \mathrm{~b}+$ macrophages in intraperitoneal lipopolysaccharide-induced aberrant lymphangiogenesis and lymphatic function in the diaphragm. Am J Pathol. 2009;175:1733-1745

132. Kataru RP, Kim H, Jang C, et al. T lymphocytes negatively regulate lymph node lymphatic vessel formation. Immunity. 2011;34:96-107 


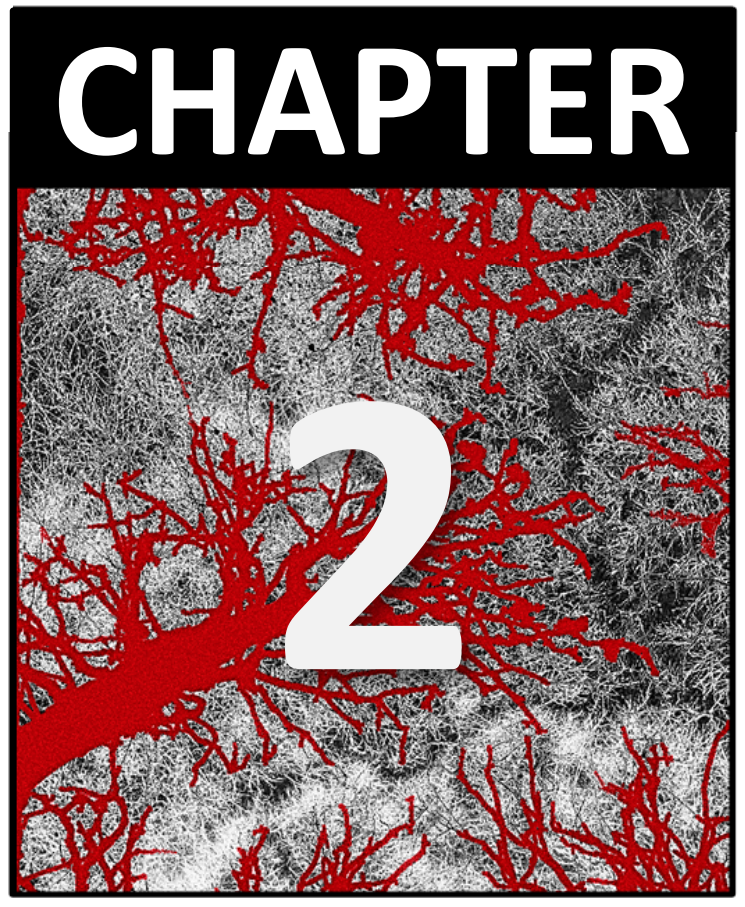




\section{Multiphoton Laser Scanning Microscopy in Atherosclerosis Research}

Zhuojun Wu, Timo Rademakers, Adelina Curaj, Mitrajit Ghosh, Fabian Kiessling, Michael Vogt, Remco T.A. Megens, Marc A.M.J. van Zandvoort 


\section{Abstract}

In the last decades, there have been great advances in the understanding of the atherosclerotic process. This has been achieved due to extensive histological research of human atherosclerotic samples, and a vast number of studies in experimental atherosclerosis models, which resulted in insight into structural and functional characteristics of plaque constituents. A great hiatus has been caused by the lack of (in vivo) whole tissue imaging techniques, allowing characterization of the dynamic processes involved in atherosclerotic plaque development and progression. Over the last decade, advances have been made in non-invasive imaging of atherosclerosis, although the techniques used, for example ultrasound, MRI, or SPECT lack the spatial resolution to examine the dynamic processes on a cellular level. Multiphoton laser scanning microscopy is an optical imaging technique allowing, albeit invasive, (sub)cellular imaging of whole tissue.

In this review, the background of multiphoton laser scanning microscopy is addressed, and advances in imaging in the light of atherosclerosis research are discussed. 


\section{Introduction}

Despite technological and diagnostic advances, the complex molecular mechanisms underlying the pathogenesis of atherosclerosis are still not fully elucidated. Although death rates from cardiovascular diseases (CVD) have been declining in recent years, it still remains one of the leading causes of morbidity and mortality in Western societies ${ }^{1}$. The majority of life-threatening cardiovascular events, including myocardial infarction and stroke, are triggered by atherosclerosis, which is characterized by a chronic inflammation of the blood vessel wall, accumulation of modified lipoproteins, macrophages, and $T$ cells, and leading to remodeling of the blood vessel walls to form atherosclerotic plaques. These ultimately may cause blockage of blood flow by thrombosis and embolization upon plaque rupture ${ }^{2}$. Thrombotic events are the major cause for atherosclerosis-related complications ${ }^{3}$.

The goal of atherosclerosis-related (vital) imaging in animals is to further unravel the complex morphological, functional, and molecular mechanisms associated with disease progression that would improve the understanding of atherogenesis and ultimately lead to novel clinical prevention, detection, and intervention strategies. Continuous innovations in noninvasive clinical imaging technologies, including magnetic resonance imaging (MRI), computed tomography (CT), nuclear imaging, fluorescence imaging, and ultrasound, provide high-resolution anatomical and functional information of the human cardiovascular system. However, in case of animal models these modalities can only define anatomical and physiological parameters. At the same time, these modalities lack the ability to provide insight on disease-related cellular and molecular processes, as each of these imaging modalities are associated with drawbacks such as, low sensitivity, low spatial resolution, and low signal-to-noise ratio ${ }^{4}$. Optical imaging techniques offer a spatial resolution superior to the conventional clinical modalities and allow not only the detailed assessment of vessel morphology, but can also provide insights on tissue composition and cellular marker expression on a molecular level. Currently, multiple optical imaging techniques such as optical coherence tomography (OCT) ${ }^{5}$, and photoacoustic tomography (PAT) ${ }^{6,7}$ are being used for imaging in human atherosclerosis. Yet, both techniques have their limitations regarding sensitivity, resolution, and penetration depth. 
Application of OCT and PAT imaging in cardiovascular tissues are well described in several other reviews ${ }^{8,9}$.

The optical imaging techniques multiphoton laser scanning microscopy (MPLSM), near-infrared (NIR) microscopic imaging, and fluorescence life time imaging (FLIM) are being applied to assess cardiovascular disease in vivo in animals and ex vivo on human tissue ${ }^{10-14}$. MPLSM has favorable properties that overcome many of the limitations of conventional single-photon imaging modalities, such as lack of out-of-focus fluorescence, better penetration depth, and reduced photo-toxicity, photo-activation, and photobleaching ${ }^{15,16}$. The combination of high spatial 3D resolution and superior penetration depth (up to $1 \mathrm{~mm}$, dependent on tissue type) has been frequently used to study intact tissue and provide information on a cellular and subcellular level ${ }^{17}$. More importantly, MPLSM allows the acquisition of functional, structural, and molecular information simultaneously in intact tissue and organs, which offers the opportunity to study processes in their physiological environment. This is particularly important for atherosclerosis, a disease associated with both structural and functional alterations ${ }^{18}$. Besides classical MPLSM systems, a variety of MPLSM systems are currently being developed as the basis for image-guided diagnostics and therapies such as photodynamic therapy (PDT) and photothermal therapy (PTT).

This review will describe the principles of MPLSM and address both the advantages and limitations for its use in cardiovascular research, and will focus especially on imaging of atherosclerosis in animals and humans. Furthermore, it will highlight how its application can benefit from the development of novel imaging probes for clinical use. 


\section{Principles of multiphoton laser scanning microscopy}

Nonlinear optical imaging has many facets. Besides the usage of conventional dyes for tissue staining, nonlinear imaging allows the visualization of tissue with intrinsic properties by exploiting second and third harmonic generation.

\section{Multiphoton excitation}

Multiphoton microscopy is based on the localized nonlinear excitation of fluorescence, which is the emission of a photon by a fluorophore activated by the absorption of multiple photons. During the absorption process, the fluorophore reaches the excited state from which it returns to the ground state by photon emission. While in conventional wide-field fluorescence or confocal laser scanning microscopy, the fluorophore-excitation is achieved with a single photon using a continuous laser, multiphoton excitation, twophoton excitation in this example (Figure 1), only occurs when a fluorophore absorbs two photons each with half the energy necessary for excitation, nearly simultaneously (within $\left.10^{-18} \mathrm{~s}\right)^{19}$. For example, a fluorophore can be excited by two photons from the near-infrared (NIR) range $(800-1000 \mathrm{~nm})$, each having half the energy of a single photon with a wavelength of $400 \mathrm{~nm}$. As the probability of such a nonlinear excitation event is rather low, a high photon density is required, which is provided by a powerful femtosecond pulse laser.

While the photon output and peak power is very high during the pulse (80-100 femtoseconds), the average energy output is relatively low when compared with a continuous laser. In addition, the photon density is highest in the focal spot of the objective and deteriorates rapidly outside the focal spot, reducing out-of-focus excitation and subsequent fluorescence, without the use of an emission pinhole as in confocal microscopy. Furthermore, multiphoton excitation can generate multiple color fluorescence using the same excitation wavelength ${ }^{20}$, which subsequently can be spectrally separated into multiple channels using band pass filters. In contrast, confocal imaging requires the use of multiple laser lines for simultaneous imaging of more than one fluorophore. This causes an increase in acquisition time proportional to the numbers of wavelengths/probes, or inefficient detection of fluorophores due to exclusion of excitation laser light from the emitted fluorescence. 


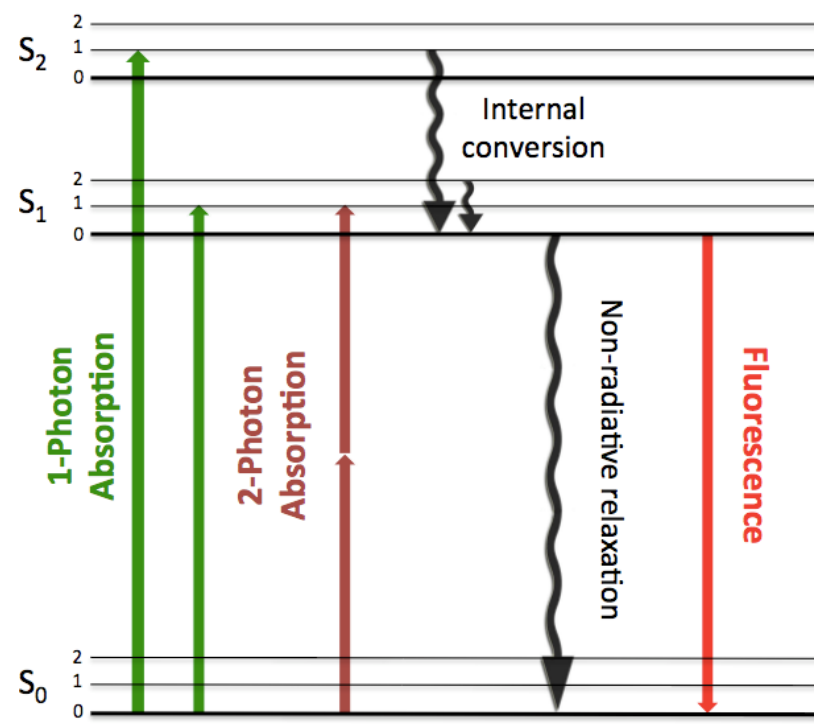

Figure 1. Principle of two-photon excitation represented in a simplified Jablonski diagram. When a fluorophore absorbs two photons with each half the energy necessary for excitation, within $10^{-18} \mathrm{~s}$, two-photon excitation is elicited and fluorescent light will be emitted.

As a result of the MPLSM layout, multiple advantages over conventional and confocal laser microscopy are evident. Firstly, the multiphoton excitation is achieved using low-energy photons in the NIR spectrum, which decreases tissue absorption and scattering and greatly increases the penetration depth. Secondly, the probability of multiphoton excitation is the highest at the focal point of the laser. Fluorophore excitation, photo-damage, photo-toxicity, and photo-bleaching are confined only to the focal point, whereas the rest of the specimen is only exposed to harmless NIR light. Thirdly, the lack of a pinhole allows the detection of scattered and unscattered emitted fluorescence for image formation, resulting in higher sensitivity, reduced excitation laser power/tissue damage and a better signalto-noise ratio. Lastly, although a high photon density is needed for multiphoton excitation, the high-energy output is only maintained for 80-100 femtoseconds. The average energy output of the multiphoton laser is much lower than continuous lasers, which reduces photodamage. One of the most prominent applications of MPLSM is deep tissue 3D imaging, during which a series of sequential images is acquired repeatedly at increasing depth to form 3-dimensional image stacks (z-stack). 
All these features are important for long-term vital deep tissue imaging, for example imaging of tumor angiogenesis ${ }^{21}$, cell tracking studies ${ }^{22,23}$, embryodevelopment ${ }^{24}$ or the progression of atherosclerosis.

\section{Second harmonic generation}

Many biological tissues contain endogenous fluorophores, which can be exploited to provide additional information on cellular activity and tissue composition, such as nicotinamide adenine dinucleotide phosphate (NAD $(P) H)$, flavin adenine dinucleotide (FAD) and extracellular matrix components ${ }^{25,26}$. A different phenomenon in optical imaging, referred to as second harmonic generation $(\mathrm{SHG})$, is frequently used for label-free imaging ${ }^{27}$. Contradictory to the multiphoton excitation process, in SHG two photons interact simultaneously with a non-centrosymmetric target, e.g. collagen fibrils leading to the emission of a single photon with exactly twice the energy (frequency doubling). Consequently, with an excitation wavelength of $800 \mathrm{~nm}$, the SHG signal is $400 \mathrm{~nm}$ (blue) ${ }^{28}$. As SHG is not dependent on the ground/excited states of molecules, i.e., SHG is a scattering process, it provides photon emission without photo-bleaching, which is of great advantage for ex vivo and in vivo imaging.

\section{Third harmonic generation}

Similar to SHG, third harmonic generation (THG) is also a nonlinear process, which requires the simultaneous interaction of three photons for the emission of a single photon with one third of the excitation wavelength. As a result, an excitation wavelength of $1200 \mathrm{~nm}$ is required for THG imaging at $400 \mathrm{~nm}$. The major difference to SHG is that THG does not require a non-centrosymmetric environment for photon emission. Refraction index change within the focal spot induces THG. However, standard multiphoton microscopes have a limited wavelength spectrum and do not allow THG, unless equipped with an Optical Parametric Oscillator (OPO). Since it is at current not used in atherosclerosis imaging, THG will not be further discussed in this review. 


\section{Imaging of atherosclerosis}

The application of MPLSM for atherosclerosis imaging offers a unique perspective. Superior tissue penetration combined with molecular, structural and functional imaging on a subcellular resolution enables the visualization of biomarkers, morphological changes, and signal molecules. All these characteristics are essential for the understanding of disease progression and the development of adequate intervention and prevention strategies.

\section{Ex vivo MPLSM imaging}

In the last few years MPLSM has been applied successfully on variety of viable tissues ex vivo, such as brain ${ }^{29-31}$, kidney ${ }^{32-36}$, lymphoid tissue ${ }^{37-41}$, tumors $21,42,43$, and heart tissue ${ }^{44,45}$. The imaging of viable blood vessels ex vivo is frequently done using perfusion chambers, in which intact isolated vessels are mounted on glass pipettes, pressurized, and corrected for longitudinal tension ${ }^{46}$. In the absence of these environmental factors, the blood vessel collapses and contracts, often leading to the loss of functionality and structural integrity 47,48 . The application of transluminal pressure, longitudinal tension and physiological buffer preserves vessel wall integrity and mimics the intact blood vessel in its physiological environment, in which not only 3D sectioning of the vascular wall and structural and functional vessel characteristics can be determined up to several hours ${ }^{46}$. Moreover, applications of specific labeling agents can be performed extraluminal, intraluminal, or both for improved labeling specificity. Together with the enhanced uptake of agents (which is especially of importance for the endothelium monolayer), mounting of arteries enhances the labeling characteristics. As such, mounting of arteries is an elegant and well controllable method for investigating the binding and uptake kinetics of multimodal probes and contrast-agents under flow and shear stress conditions, such as fluorescent microbubbles ${ }^{49}$, magneto-optical nanoparticles ${ }^{50}$, and contrast agents $^{51}$.

\section{Imaging of second harmonic generation signal}

In biological tissue, the extracellular matrix mainly contributes to SHG due to the high content of non-centrosymmetric structures such as collagen fibers. Typical for blood vessel walls, most of the extracellular material between the 
smooth muscle and in the adventitia is collagen, which is responsible for vascular integrity and stability ${ }^{52,53}$. Moreover, collagen plays an important role in the progression of atherosclerotic plaques and other cardiovascular diseases such as hypertension and age-related arterial stiffness ${ }^{54-56}$. Its extensive contribution to and impact on vascular remodeling and plaque development has been reviewed by Adiguzel et al in $2009^{57}$.

Collagen has been the focus of many cardiovascular-related studies using MPLSM ${ }^{58-61}$. The complex mechanisms and dysfunctional balance of collagen synthesis and degradation during atherogenesis has a major impact on disease progression. While collagen content can spike up to $60 \%$ of total plaque proteins, therefore contributing significantly to plaque growth and arterial narrowing in fibrous lesions. Alternatively, collagen degradation in the fibrous cap makes the plaque vulnerable and prone to rupture. It should be noted, however, that only specific types of collagen such as collagen I, III, IV, V, and VIII along the subendothelial layer are actively involved in plaque development and rupture ${ }^{57,62}$.

\section{Imaging of disease-specific markers}

In addition to SHG and THG, the combination of high-resolution and -sensitivity imaging and deep tissue penetration of MPLSM has yielded insights into the structural, functional, and molecular alterations in the arterial wall prior to lesion development and the composition of atherosclerotic plaques in the later stages of disease progression.

The detection of molecular disease markers such as inflammatory receptors requires a method with high sensitivity, as their concentration is expected to be in the nanomolar range. This is especially true for markers in the subendothelial space, as probes will only limitedly penetrate the intima. Commonly used imaging modalities such as molecular ultrasound ${ }^{63-65}$, PET ${ }^{66,67}, \mathrm{CT}^{68}$, and MRI ${ }^{69,70}$ all lack the capability for the visualization of (sub)cellular structures. Multiphoton imaging using fluorescence and NIR excitation, has shown its potential as a high-resolution and highly sensitive imaging method for atherosclerosis in preclinical studies in small animals, both ex vivo and in vivo ${ }^{11,12,15,18,51 .}$

One of the targets for imaging of early stages of atherosclerosis development is endothelial dysfunction, which is considered one of the key events in the onset of atherogenesis. The dysfunction of both barrier and 
secretory functions is accompanied by the luminal presence and release of chemokines and cytokines ${ }^{71}$, and overexpression of cell adhesion molecules, such as intercellular adhesion molecule (ICAM), vascular cell adhesion molecule (VCAM), platelet endothelial cell adhesion molecule (PECAM/CD31), and selectins ${ }^{72}, 73$. These processes cause an increased accumulation of inflammatory cells, such as monocytes, neutrophils, and T cells, as well as lipids in the vessel wall. Both cell adhesion molecules and chemokines/cytokines can potentially be used as molecular markers for early stages of lesion development using optical imaging techniques as demonstrated in multiple studies with fluorescent labeled multimodal probes, i.e. VCAM-1 labeled nanoparticles or P-selectin labeled microbubbles ${ }^{74-78}$.

Prominent molecular markers for the later stages of lesion development are inflammatory- and angiogenesis-related receptors such as $\mathrm{CD} 13^{79}$ or $\alpha_{v} \beta_{3}$ integrin ${ }^{80}$ for studying plaque neovascularization. Furthermore, extracellular matrix (ECM)-related proteins such as collagen and endothelial fibrin $58,81,82$ could serve as markers for studying cap formation and cap thinning, and thrombus-related factors like activated platelets ${ }^{83,84}$ may serve as markers for intraplaque hemorrhage (IPH) or plaque rupture. In addition to the disease-related molecular markers, MPLSM allows the visualization of functional alteration in the murine vascular wall typical for atherogenesis such as NO production (Ghosh et al, submitted), structural changes in the vascular wall typical for atherogenesis such as angiogenesis ${ }^{15}$, endothelial glycocalyx thickness and structure ${ }^{10,85}$, collagen levels ${ }^{58}$, and morphological changes such as the vessel diameter and vessel wall thickness ${ }^{86}$.

Functional imaging of atherosclerosis using MPLSM has only recently been explored by visualizing NO production within diseased tissue ex vivo (Ghosh et al, submitted). The altered NO production as a consequence of endothelial dysfunction results in a number of cardiovascular symptoms such as hypertension and atherosclerosis ${ }^{87}$. The combination of an NO-sensitive fluorescence probe and MPLSM has provided valuable information regarding the structural-functional relationship of NO production in the vessel wall with direct visualization of the structures producing NO, shedding light onto the role of NO during vascular dysfunction.

In addition to the molecular and functional imaging possibilities, the strong tissue penetration of MPLSM allows structural imaging of the components of the (intact) vessel wall. The media consists mostly of layers of 
smooth muscle cells separated by elastin fibers, while the major component of the most outer adventitia layer is collagen ${ }^{88}$. During lesion progression, the vessel wall undergoes structural remodeling steps, such as the increase of the intima-media thickness (IMT), alteration of the endothelial glycocalyx thickness ${ }^{10,85}$, increased smooth muscle cell proliferation, and collagen synthesis. As demonstrated in a study by Megens et al in 2007, the collagenmarker CNA35 was characterized and established using MPLSM, as a molecular marker for atherosclerotic plaques in $\mathrm{ApoE}^{-/}$mice, mainly based on the endothelial uptake and distribution of the collagen-marker. Due to characteristic increase of endothelial permeability and elevated collagen content in the lesion areas, CNA35 showed significantly higher binding compared to healthy tissue.

The characterization of novel molecular probes using MPLSM imaging provides both functional and structural information on a cellular and subcellular level and may contribute to the development of multi-modal agents for noninvasive clinical imaging modalities with shortcomings in spatial resolution or sensitivity, such as molecular ultrasound, MRI, CT, and PET/SPECT.

\section{In vivo MPLSM imaging}

Despite significant improvements of in vitro and ex vivo models for atherosclerosis, preclinical animal experiments are still crucial for understanding disease processes, effects of new drugs, and contrast agents. So far, in vivo multiphoton laser scanning microscopic imaging has been mostly performed in relatively motionless/fixed tissue, and has been applied for studying renal (patho)physiology ${ }^{89-92}$, tumor biology ${ }^{93,94}$, and angiogenesis ${ }^{95}$. Other fields implementing in vivo MPLSM techniques are ophthalmology ${ }^{96}$, dermatology ${ }^{97}$, pulmonary research ${ }^{98}$, and neurosciences ${ }^{99-102}$. The field of immunology has also greatly profited from in vivo MPLSM, where it has led to new insights in e.g. haematopoietic stem cells and their trafficking ${ }^{103}$, immune cell recruitment ${ }^{104-107}$, immune cell trafficking ${ }^{108,}{ }^{109}$, and infection in general ${ }^{110}$. Most in vivo MPLSM of the vasculature has been focusing on imaging of microvasculature in various tissue beds and organs and is mainly utilized for determining e.g. flow in the brain ${ }^{111}$ or in tumors ${ }^{112}$, cell trafficking ${ }^{113,}{ }^{114}$, permeability ${ }^{115}$ and contractility ${ }^{116}$, coagulation ${ }^{117}$, or structural composition and aberrations (e.g. of the glycocalyx) ${ }^{10,118}$, and the 
process of angio- and atherogenesis ${ }^{119}$. Yet, the macrovasculature, among others important for studying atherosclerosis or related processes, has not been extensively studied in vivo with MPLSM because of practical difficulties concerning image quality.

Initial studies of the microvasculature focused mainly on coping with motion artifacts and were used for structural imaging of the vessel wall ${ }^{120}$ and to study interactions of leukocyte subsets with the endothelium ${ }^{11,12}$. The in vivo motion artifacts are mainly caused by both the cardiac and respiratory cycle of living specimen which results in movement of tissue beds with a certain frequency. In case of mice, the heart frequency is roughly 5-6 $\mathrm{Hz}$ and the respiratory cycle 2-4 $\mathrm{Hz}$ (dependent on applied anesthesia). Consequently, the thoracic area, the myocardium, and the larger arteries will display a rhythmic motion with similar frequencies. As a result, imaging of these tissue beds in vivo with a laser scanning microscope, strongly suffers from two types of artifacts: intra-frame and inter-frame motion artifacts. The intra-frame motion artifacts are a result of the laser scanning properties of MPLSM, where there is a gradient in time difference between the first scanned pixel in the left top corner of the image matrix and the last scanned pixel at the right bottom corner. As such, the tissue in the 2-3 $\mu \mathrm{m}$ thin focal plane varies within the single image and the motion of the tissue causes a deterioration of the image due to the actual motion and loss of focus (Figure 2$)^{18}$. In an imaging sequence (either in depth or over time), the motion of tissue also causes a difference of the tissue in focus between subsequent images. As a result, (three dimensional) reconstructions of cellular processes or structures are almost impossible to perform without adapting the current imaging methods in order to limit or overcome motion artifacts.

Besides the sometimes rather non-physiological procedures of physical suppression of the tissue motion either by adapting the surgical preparation method ${ }^{121}$, or temporary reduction of the heart rate or respiration ${ }^{122}$, there are three strategies to (partially) overcome both artifacts: 1) utilize the cardiac and respiratory cycle to guide the imaging by timing of the MPLSM acquisition in a time window where the motion will be limited; 2 ) trigger the acquisition by using the cardiac and respiratory signals (triggered acquisition); 3) record the moving tissue randomly, and apply extensive post-processing of the images series to detect comparable tissue representations over time. 


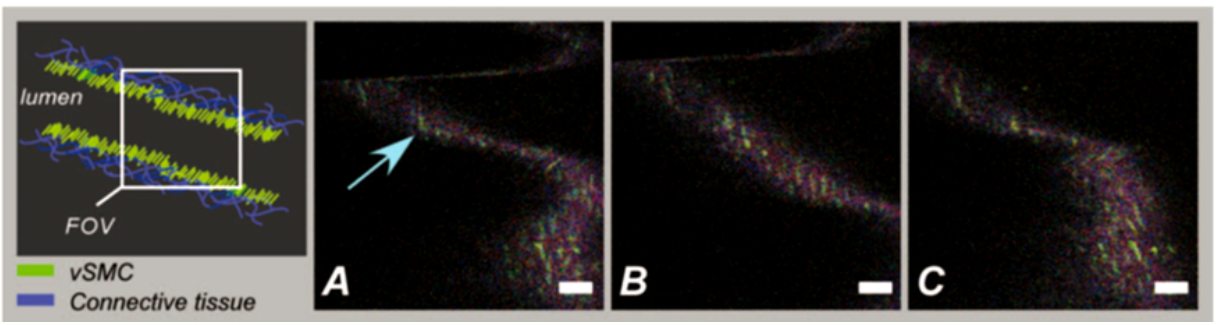

Figure 2. Motion artifacts and loss of focus in untriggered in vivo MPLSM imaging ${ }^{18}$. Three subsequent optical sections $(A-C)$ of a left carotid artery of a C57B/6/J mouse obtained in vivo without application of external triggering. Frame rate was $2.3 \mathrm{~Hz}$ (1200 Ips; line scan rate 1X, matrix size $512 \times 512$ pixels). Drawing indicates the position of the optical sections in the vessel wall; bars indicate $20 \mu \mathrm{m}$. Cell nuclei (SYTO13, green) and collagen (SHG, blue/purple) are visible. All sections are disturbed by motional artifacts which cause the arterial wall (blue arrow) to appear as a curved-like structure. Moreover, every optical section contains different parts of the (moving) vessel wall. The VSMC nuclei (green), with their typical morphology and orientation, are hardly recognizable; the position of the lumen is unclear.

\section{Respiratory timing}

In order to (partially) overcome in vivo motion artifacts, timing of the image acquisition between the tissue movements is a logical step. When the image acquisition frequency is higher than the movement frequencies, in principle one can record a single image without intra-image artifacts. Timing of image acquisition has been performed for imaging of the lung using intravital and laser scanning microscopy ${ }^{123}$. By mechanically controlling the respiration, a stable respiration motion frequency is obtained that allows image acquisition in between the respiration frequency without intra-image artifacts (only when acquisition frequency is higher than motion frequency). Additionally, when the motion frequency is very stable and acquisition frequency is high enough, it will be possible to overcome inter-image artifacts and as a result potentiate studying of structures and or processes in 3D over time.

\section{Respiratory triggering}

When motion artifacts are caused by more than one determinant or the motion frequencies are less stable and consequentially difficult to predict, it is virtually impossible to avoid motion artifacts by simple timing of acquisition. In such tissue beds, image acquisition can be timed by gating / triggering on the main determinants of the sample motion, a technique applied for other imaging modalities such as MRI ${ }^{124}$. Gating, or triggered acquisition, makes it feasible to overcome a combination of motion artifacts when using a triggering 
tool that enables generation of trigger pulses based on more than one signal. Moreover, it offers the advantage that smaller motional irregularities can be blocked using appropriate trigger thresholds or trigger delays and as such do not contribute to the resultant data set.

In case of studying cardiovascular samples such as the large arteries, the motion disturbances are a consequence of the cardiac $(5-6 \mathrm{~Hz})$ and respiratory $(2-4 \mathrm{~Hz})$ cycle, from which the cardiac cycle is the most prominent motion disturbance ${ }^{18}$. In this study, utilization of a gating- or triggerbox (Rapid biomedical, Würzburg, Germany), originally developed for MRI systems, combined with image acquisition at a rate of 5-6 Hz resulted in more stable image series of murine carotid and renal arteries without large intra- and interimage artifacts caused by motion. However, in order to achieve the required acquisition rates and the detection sensitivity, the number of pixels had to be lowered and the scan speed increased. As a result, the image quality suffered due to a degraded signal-to-noise ratio. Moreover, the resolution is reduced since the Nyquist sampling criterion is not met due to the reduced number of voxels (a consequence of the mismatch between digital and optical resolution: optical resolution / pixel size $\leq 2.3$ ).

In the latest generation of commercially available MPLSM systems, overall sensitivity has drastically improved due to strongly enhanced optics (apochromatic objectives with high numerical aperture and low magnification) and acquisition rates of $>30 \mathrm{~Hz}$ using resonance scanning $\left(8000-12000 \mathrm{~Hz},{ }^{125}\right)$ or multiplication of the excitation laser beam (1-64 beams ${ }^{126,127}$ ), improving time resolution. The latter enables recording at video rates without a negative impact on the overall image quality, size of the field of view, or the image resolution. Moreover, novel fluorophores with improved quantum yields and stokes-shift contribute to improvement of image quality ${ }^{128}$. As a consequence, the intra- and inter-image artifacts can be strongly reduced, allowing more accurate gating with strict thresholds, and ultimately, enable recording of $>1$ optical slice within the time window of tissue stability. Due to the real-time nature of motion depression by gated image acquisition, this technique holds potential for obtaining three dimensional datasets over time in strongly motional tissues since subsequent images may be obtained at exactly the same starting point within the motion cycle(s). By controlling the tissue in focus (preferably by a piezo- or galvo- controlled z-drive for fast focusing), accurate three-dimensional datasets may be obtained. 


\section{Fast scanning imaging}

Besides timed or gated imaging, one can record the moving tissue over time at a $10-30 \mathrm{~Hz}$ acquisition rate without triggering or other hardware aids. The resultant dataset consists of optical slices where motional artifacts are captured with a frequency that matches or exceeds the frequency of the heart respiration rate (Figure 3 ). Such datasets can be subjected to extensive postprocessing of the images series in order to detect comparable tissue representations over time. In most cases, the (partially) stable images have a rhythmic appearance (dependent on motion and acquisition frequency) within the data set which is beneficial for the selection process. Secondly, non-moving structures or cells within the selected images may be compared using overlays, edge detection, or co-localization protocols; The matching images may then be used for analyses of biological structures ${ }^{12}$ or cell recruitment ${ }^{11,129}$. Post processing of the acquired data may be further optimized by making use of the signal recordings of the motion disturbances (e.g. electrocardiography or respiratory signals). Moreover, while the post-processing method can be performed manually, a more automated (structure recognition based) image processing is preferential since the process is extremely time consuming and has an unclear outcome (are there enough stable images within the dataset? Is the quality good enough to trace cells or study structures over time?).

Another point of attention is the vulnerability of manual post processing for intra-observer artifacts. Finally, in order to make post processing corrected in vivo imaging more mainstream, there is a need for (development of) automatic processing protocols to analyze non-gated in vivo datasets that make use of pattern recognition algorithms and incorporate (real-time) knowledge of the motion frequency (i.e. heart and respiration rates). Besides improved quality, automatic in vivo post processing will enable more researchers to apply laser scanning microscopy in strongly motional samples and eventually, may generate novel data on the behavior of structures under in vivo motion (for example arterial wall deformation as a result of the blood pressure).

As is evident, all three techniques (respiratory timing, respiratory triggering and fast scanning imaging) have their advantages and disadvantages, and the choice for one of the three will also depend on the research question at hand. Studies focused on e.g. tissue structure would greatly profit from the most stable imaging, requiring triggering whereas fast processes may benefit 


\section{CHAPTER 2}

more from continuous imaging, e.g. tracking of cells in the arterial system, since triggering-based techniques would result in too slow acquisition for proper assessment of real-time dynamics. In general, the quality of the in vivo image series is strongly dependent on the stability of the sample preparation (i.e. tissue drifts or loss of immersion water may cause a shift of the in-focus tissue) and the stability of the subject under investigation (method of anesthesia, heart rate, blood pressure, body temperature). Independent of the technique used, in vivo multiphoton imaging has become an important imaging tool over the years, and has given new insight in different fields of research.

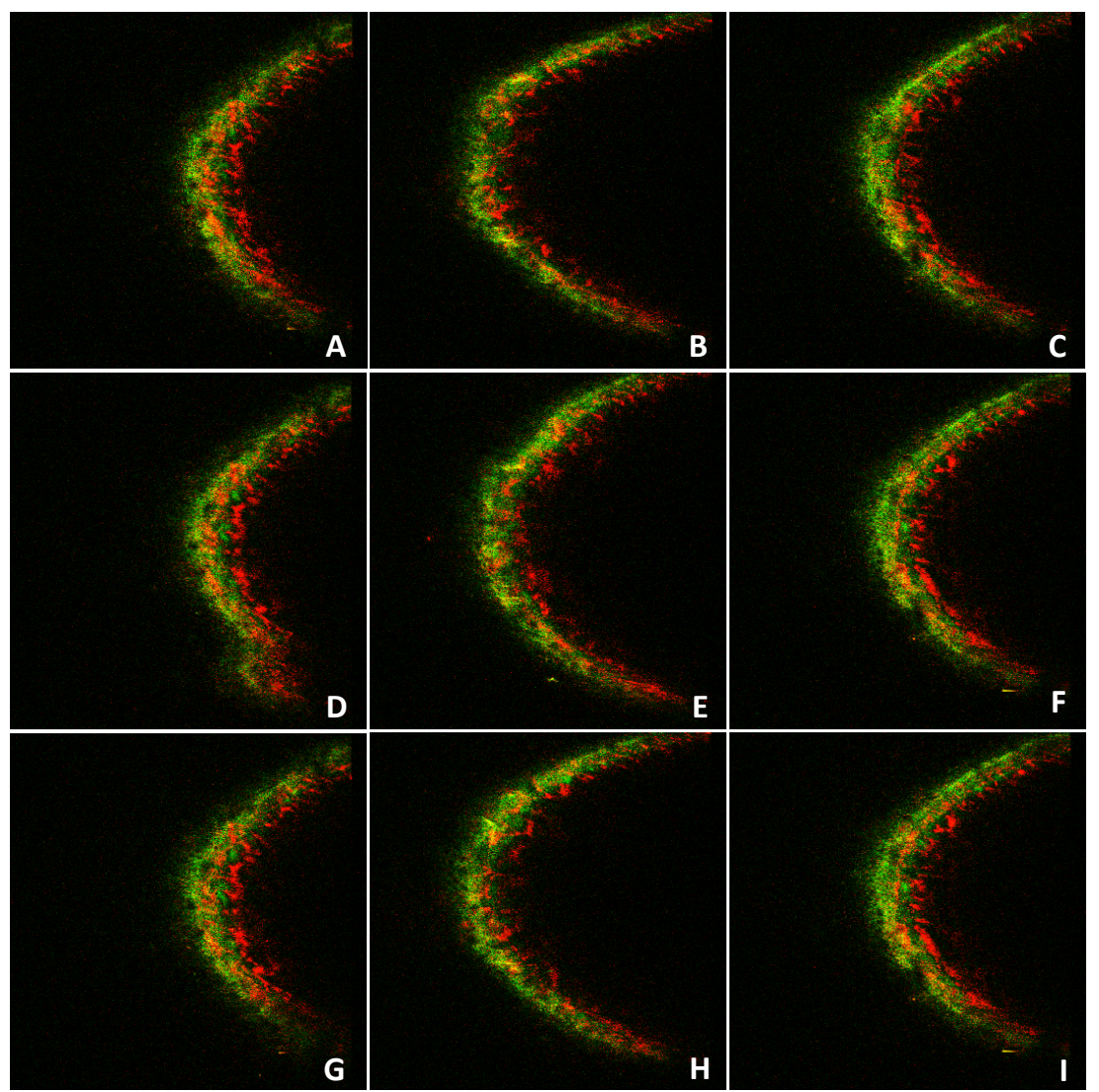

Figure 3. Fast scanning imaging allows stable imaging of moving tissue. Nine consecutive optical sections (A-I) from a murine carotid artery are shown with vascular endothelial marker CD31 (red) and elastin autofluorescence (green). A rhythmic appearance can be appreciated. 


\section{Heart imaging}

In vivo multiphoton imaging of the heart has been gaining interest since many fields of research, also atherosclerosis research, would benefit greatly from subcellular imaging of the heart in vivo. Yet, the heart is being more affected by severe motion artifacts than any other organ, and as such it is one of the most challenging organs to apply in vivo multiphoton microscopy on. Only recently, a first attempt has been made to visualize a beating mouse heart at a subcellular resolution. Li et al were able to perform in vivo MPLSM imaging on a beating heart using a system involving heterotopic transplants and an imaging chamber ${ }^{130}$. In vivo imaging of the heart has been further refined by Lee et $a^{131}$, who elegantly combined existing triggering methods with a heart stabilizing tool that suppresses part of the motion of the beating heart inside the pericardial cavity. The impressive result is high resolution imaging of the intact in vivo mouse heart for studies with a duration of $>4$ hours. It can be expected that especially the latter method will prove to be a powerful tool for in vivo studies of the heart and will strongly contribute to our understanding of its morphology, physiology, and pathophysiology, especially when combined with systems allowing even faster acquisition rates and more sensitive detection. 


\section{Novel imaging agents}

New advances in imaging agents are very important factors in successful in vivo imaging. In general, high quantum yields are favorable, especially when studying fast processes. In vivo MPLSM imaging has therefore significantly benefited from the introduction of new nanoparticles, as has been reviewed extensively ${ }^{128,132}$. Aside from the traditional probes, especially quantum dots have been widely studied for in vivo MPLSM imaging. Their stability, high quantum yield and narrow emission spectra have made them excellent candidates for achieving a high signal-to-noise ratio in tissues suffering from intra-frame and inter-frame motion artifacts, while at the same time allowing multicolor imaging. For long-term studies, quantum dots are still debated due to the lack of clearance and concomitant degradation and release of toxic constituents inside the organism. Novel methods which are focused on lowering the toxicity of quantum dots will prove helpful in future studies ${ }^{133,134}$. Yet, (in vivo) MPLSM imaging has profited as well from development of more advanced probes, e.g. multimodal probes or photo-activatable, photo-convertible or photo-switchable probes. Multimodal probes are imaging agents designed to be detectable by two or more imaging modalities, like combinations of MPLSM with MRI ${ }^{50,51,135,136}$, OCT (reviewed by Tang et al ${ }^{137}$ ), Fluorescence Molecular Tomography (FMT) ${ }^{138}$, and Contrast-Enhanced Ultrasound (CEUS) ${ }^{49}$. Such probes have made it possible to examine biodistribution and gross anatomy using (non-invasive) imaging techniques, and afterwards using MPLSM imaging to determine localization of the probe at (sub-)micron resolutions. The most prominent problem with multimodal imaging, however, still encompasses the integration of the different techniques. Further integration, as reviewed for MPLSM and OCT ${ }^{137}$, is needed, but will, in the future, facilitate actual multimodal imaging in vivo.

Photo-activatable, -convertible or -switchable probes have been developed over the last decade and are very suitable for studying protein dynamics in live-cell imaging ${ }^{139-142}$. Dependent on the exact type of probe, it is non-fluorescent and can become fluorescent (photoactivation), is fluorescent and irreversibly shifts fluorescence emission (photoconversion) or is fluorescent and reversibly shifts fluorescence emission (photoswitching). The transition is traditionally accomplished by ultraviolet (UV) illumination, 
changing the molecular structure of the fluorescent molecule, but can also be achieved by multiphoton excitation, where the activation wavelength and excitation wavelength for imaging obviously need to be separated. More recently, these probes have been applied as a cell tracking tool for immunologic responses in microvasculature ${ }^{143-145}$, and are bound to be applied in the microvasculature in the near future.

An additional novel development in probes and probe detection is the use of wavelength mixing. It is especially for lineage tracking and cell tracking, and allows imaging of multiple probes simultaneously, taking into account the relative expression of the probes. Mahou et al described the use of a MPLSM setup equipped with an OPO unit, to enable even higher near infrared excitation wavelengths $(700-1350 \mathrm{~nm})$, in combination with a Brainbow transgene approach ${ }^{146}$. Although this technique is not available for live imaging yet, it holds promise for future in vivo tracking studies.

\section{Pros and cons of MPLSM}

Imaging of viable tissue using MPLSM offers great advantages. It reduces the probability of artifacts that accompany the various steps of tissue processing required for histology and immunohistochemistry (cutting, fixation, staining) ${ }^{147}$ since penetration depth of MPLSM allows imaging of structures and function in intact large arteries or organs. The penetration depth of light is strongly dependent on tissue type, as each tissue composition shows unique absorption and scattering profiles ${ }^{148,}{ }^{149}$, which make conventional fluorescence imaging methods less effective in deeper tissue layers. In atherosclerotic lesion areas, penetration depth is heavily restricted due to calcification and the accumulation of tissue components with high scattering coefficients such as cholesterol esters. Both scattering and absorption are wavelength-dependent and decrease with longer excitation wavelength. Yet, the emitted fluorescence still is in the range of normal fluorescence microscopy and thus suffers equally from scattering and absorption. The wavelengths used in TP imaging are within the limits of the NIR transparency window $(700-1100 \mathrm{~nm})$ of biological tissues, showing reduced absorption and thereby maximizing tissue penetration. Moreover, the lack of pinholes positively influences the detected emission, which is beneficial for sensitivity and imaging at depth. 
In general, MPLSM is favored over conventional wide field and confocal microscopy due to its ability to penetrate deeper into biological tissues with reduced deterioration of spatial resolution. Nevertheless, MPLSM has some drawbacks. Firstly, the use of NIR excitation results in a larger resolution spot for MPLSM, therefore the spatial resolution of confocal microscopy supersedes that of MPLSM at shallow depths ${ }^{150}$. Secondly, although photo-toxicity is confined to the focal spot and reduced, TP excitation can still have a negative impact on tissue viability. High photon density and laser power during the excitation period of the femtosecond laser can cause photo- damage to the specimen, potentially leading to oxidative stress and ultimately cell death ${ }^{151}$. Thirdly, contradictory to conventional brightfield-fluorescence intravital microscopes, which use CCD cameras for acquisition, MPLSM image acquisition is based on point-to-point scanning of the field of view. As a result, imaging rate in classical MPLSM systems ( $\pm 20 \mathrm{fps}$ ) is still reduced and can lead to motion artifacts in in vivo imaging studies. This can be compensated by using timing, triggering systems or fast, resonance or multi-beam based MPLSM imaging systems. However, it is important to note that the signal-to-noise ratio decreases with the scanning frequency, emphasizing the need for highlysensitive detectors. Fourthly, the ability to provide multiphoton excitation and subcellular resolution is associated with usage of high numerical aperture objectives and therefore often has a relatively small field of view. The latter is limiting the potential of imaging whole animals. Finally, one of the major drawbacks and the reason why MPLSM has not been fully integrated into laboratory routine is its high price and the requirement for specialized personnel to run the system ${ }^{152}$.

\section{Conclusion}

MPLSM is a still rapidly evolving tool that has already shown its feasibility for various research fields. Its main strength to maintain resolution within various tissue types permits the visualization of cellular structures with high resolution $( \pm 1 \mu \mathrm{m})$, which has helped to characterize and establish novel imaging agents for atherosclerosis, as well as shed light into the molecular mechanisms of vascular remodeling and atherosclerotic lesion development. Furthermore, low photo-toxicity of MPLSM imaging supports long-term in vivo studies in the same animals, which is essential for the characterization of a slow-progressing 
disease such as atherosclerosis. Advances in triggering-systems and highfrequency optical scanners will further improve imaging-rate and image quality of MPLSM, allowing precise structural, functional, and molecular analysis in vivo.

\section{Future directions}

In many cases, the ultimate goal of exploratory work in multiphoton imaging is to translate those insights towards the development of in vivo and clinical diagnostic and therapeutic tools. Although the feasibility of in vivo applications for cardiovascular research has been demonstrated in animals, to our knowledge there have been no reports on its application on human subjects. Until now, clinical multiphoton imaging has almost exclusively been used on human skin both ex vivo and in vivo ${ }^{153-155}$. Due to limited light penetration through the skin, in vivo cardiovascular MPLSM imaging requires procedures to expose the tissue of interest and create a surgically embedded optical window or the application of an endoscope.

As already developed and established a decade ago, the combination of fluorescence imaging and endoscopy offers the possibility of non- or minimal invasive imaging in humans ${ }^{156}$. Contradictory to the high numbers of single-photon confocal mircoendoscopy studies in humans and increased usage in clinical routine ${ }^{157-160}$, there has been only one MPLSM study in humans ${ }^{161}$. The major challenges are the efficient delivery of femtosecond excitation light without major degradation, the wide-field collection of the multiphoton fluorescence, miniaturization of objective lenses and the endoscope itself. Recent technological innovations in photonic crystal fibers ${ }^{162}$ and imaging lenses (fiber-coupled gradient-index - GRIN lenses) ${ }^{163}$ have partially overcome these limitations. New multiphoton microendoscopes have been developed and tested in animal studies ${ }^{164-166}$ and will mostly likely contribute to the development of clinical multiphoton microendoscope prototypes and clinical studies outside of the dermatological field in the next years. 


\section{References}

1. Roger VL, Go AS, Lloyd-Jones DM, et al. Executive summary: Heart disease and stroke statistics--2012 update: A report from the american heart association. Circulation. 2012;125:188-197

2. Ross R. Atherosclerosis--an inflammatory disease. $N$ Engl J Med. 1999;340:115-126

3. van der Wal AC, Becker AE. Atherosclerotic plaque rupture--pathologic basis of plaque stability and instability. Cardiovasc Res. 1999;41:334-344

4. Ransohoff KJ, Wu JC. Advances in cardiovascular molecular imaging for tracking stem cell therapy. Thromb Haemost. 2010;104:13-22

5. Liu L, Gardecki JA, Nadkarni SK, et al. Imaging the subcellular structure of human coronary atherosclerosis using micro-optical coherence tomography. Nat Med. 2011;17:1010-1014

6. Jansen $\mathrm{K}$, van der Steen $\mathrm{AF}$, van Beusekom $\mathrm{HM}$, et al. Intravascular photoacoustic imaging of human coronary atherosclerosis. Opt Lett. 2011;36:597-599

7. Wang B, Su JL, Amirian J, et al. Detection of lipid in atherosclerotic vessels using ultrasound-guided spectroscopic intravascular photoacoustic imaging. Opt Express. 2010;18:4889-4897

8. Bezerra HG, Costa MA, Guagliumi G, et al. Intracoronary optical coherence tomography: A comprehensive review clinical and research applications. JACC Cardiovasc Interv. 2009;2:1035-1046

9. Wang B, Karpiouk A, Yeager D, et al. Intravascular photoacoustic imaging of lipid in atherosclerotic plaques in the presence of luminal blood. Opt Lett. 2012;37:1244-1246

10. Reitsma S, Oude Egbrink MG, Heijnen VV, et al. Endothelial glycocalyx thickness and platelet-vessel wall interactions during atherogenesis. Thromb Haemost. 2011;106:939-946

11. Drechsler M, Megens RT, van Zandvoort M, et al. Hyperlipidemia-triggered neutrophilia promotes early atherosclerosis. Circulation. 2010;122:1837-1845

12. Megens RT, Vijayan S, Lievens D, et al. Presence of luminal neutrophil extracellular traps in atherosclerosis. Thromb Haemost. 2012;107:597-598

13. Phipps J, Sun Y, Saroufeem R, et al. Fluorescence lifetime imaging for the characterization of the biochemical composition of atherosclerotic plaques. $J$ Biomed Opt. 2011;16:096018

14. Park J, Pande P, Shrestha S, et al. Biochemical characterization of atherosclerotic plaques by endogenous multispectral fluorescence lifetime imaging microscopy. Atherosclerosis. 2012;220:394-401

15. van Zandvoort M, Engels W, Douma K, et al. Two-photon microscopy for imaging of the (atherosclerotic) vascular wall: A proof of concept study. J Vasc Res. 2004;41:54-63

16. Cahalan MD, Parker I, Wei SH, et al. Two-photon tissue imaging: Seeing the immune system in a fresh light. Nat Rev Immunol. 2002;2:872-880 
17. Helmchen F, Denk W. Deep tissue two-photon microscopy. Nat Methods. 2005;2:932-940

18. Megens RT, Reitsma S, Prinzen L, et al. In vivo high-resolution structural imaging of large arteries in small rodents using two-photon laser scanning microscopy. J Biomed Opt. 2010;15:011108

19. Denk W, Strickler JH, Webb WW. Two-photon laser scanning fluorescence microscopy. Science. 1990;248:73-76

20. Xu C, Williams RM, Zipfel W, et al. Multiphoton excitation cross-sections of molecular fluorophores Bioimaging. 1996;4:198-207

21. Tanaka K, Morimoto $\mathrm{Y}$, Toiyama $\mathrm{Y}$, et al. In vivo time-course imaging of tumor angiogenesis in colorectal liver metastases in the same living mice using twophoton laser scanning microscopy. J Oncol. 2012;2012:265487

22. Levi V, Ruan Q, Gratton E. 3-d particle tracking in a two-photon microscope: Application to the study of molecular dynamics in cells. Biophys J. 2005;88:2919-2928

23. Matheu MP, Cahalan MD, Parker I. Immunoimaging: Studying immune system dynamics using two-photon microscopy. Cold Spring Harb Protoc. 2011;2011:pdb top99

24. Squirrell DJ, Wokosin D, White JG, et al. Long-term two-photon fluorescene imaging of mammalian embryos without compromising vitality. Nature biotechnology. 1999;17:763-767

25. Monici M. Cell and tissue autofluorescence research and diagnostic applications. Biotechnol Annu Rev. 2005;11:227-256

26. Roberts MS, Dancik Y, Prow TW, et al. Non-invasive imaging of skin physiology and percutaneous penetration using fluorescence spectral and lifetime imaging with multiphoton and confocal microscopy. Eur J Pharm Biopharm. 2011;77:469-488

27. Campagnola P. Second harmonic generation imaging microscopy: Applications to diseases diagnostics. Anal Chem. 2011;83:3224-3231

28. Fine S, Hansen WP. Optical second harmonic generation in biological systems. Appl Opt. 1971;10:2350-2353

29. Svoboda K, Denk W, Kleinfeld D, et al. In vivo dendritic calcium dynamics in neocortical pyramidal neurons. Nature. 1997;385:161-165

30. Helmchen F, Waters J. Ca2+ imaging in the mammalian brain in vivo. Eur J Pharmacol. 2002;447:119-129

31. Levene MJ, Dombeck DA, Kasischke KA, et al. In vivo multiphoton microscopy of deep brain tissue. J Neurophysiol. 2004;91:1908-1912

32. Dunn KW, Young PA. Principles of multiphoton microscopy. Nephron Exp Nephrol. 2006;103:e33-40

33. Ashworth SL, Tanner GA. Fluorescent labeling of renal cells in vivo. Nephron Physiol. 2006;103:p91-96

34. Tanner GA, Rippe C, Shao Y, et al. Glomerular permeability to macromolecules in the necturus kidney. Am J Physiol Renal Physiol. 2009;296:F1269-1278

35. Yu W, Sandoval RM, Molitoris BA. Quantitative intravital microscopy using a generalized polarity concept for kidney studies. Am J Physiol Cell Physiol. 2005;289:C1197-1208 
36. Molitoris BA, Sandoval RM. Intravital multiphoton microscopy of dynamic renal processes. Am J Physiol Renal Physiol. 2005;288:F1084-1089

37. Worbs T, Mempel TR, Bolter J, et al. Ccr7 ligands stimulate the intranodal motility of t lymphocytes in vivo. J Exp Med. 2007;204:489-495

38. Zinselmeyer BH, Dempster J, Gurney AM, et al. In situ characterization of cd4+ t cell behavior in mucosal and systemic lymphoid tissues during the induction of oral priming and tolerance. J Exp Med. 2005;201:1815-1823

39. Mempel TR, Henrickson SE, Von Andrian UH. T-cell priming by dendritic cells in lymph nodes occurs in three distinct phases. Nature. 2004;427:154-159

40. Mempel TR, Scimone ML, Mora JR, et al. In vivo imaging of leukocyte trafficking in blood vessels and tissues. Curr Opin Immunol. 2004;16:406-417

41. Ng LG, Mrass P, Kinjyo I, et al. Two-photon imaging of effector t-cell behavior: Lessons from a tumor model. Immunol Rev. 2008;221:147-162

42. Tozer GM, Ameer-Beg SM, Baker J, et al. Intravital imaging of tumour vascular networks using multi-photon fluorescence microscopy. Adv Drug Deliv Rev. 2005;57:135-152

43. Douma K, Oostendorp M, Slaaf DW, et al. Evaluation of magnetic resonance vessel size imaging by two-photon laser scanning microscopy. Magn Reson Med. 2010;63:930-939

44. Buehler A, van Zandvoort MA, Stelt BJ, et al. Cngr: A novel homing sequence for cd13/apn targeted molecular imaging of murine cardiac angiogenesis in vivo. Arterioscler Thromb Vasc Biol. 2006;26:2681-2687

45. Oostendorp M, Douma K, Wagenaar A, et al. Molecular magnetic resonance imaging of myocardial angiogenesis after acute myocardial infarction. Circulation. 2010;121:775-783

46. Megens RT, Reitsma S, Schiffers PH, et al. Two-photon microscopy of vital murine elastic and muscular arteries. Combined structural and functional imaging with subcellular resolution. J Vasc Res. 2007;44:87-98

47. Boulesteix T, Pena AM, Pages $N$, et al. Micrometer scale ex vivo multiphoton imaging of unstained arterial wall structure. Cytometry A. 2006;69:20-26

48. Maffia P, Grassia G, Di Meglio P, et al. Neutralization of interleukin-18 inhibits neointimal formation in a rat model of vascular injury. Circulation. 2006;114:430-437

49. Fokong $\mathrm{S}$, Theek $\mathrm{B}, \mathrm{Wu} \mathrm{Z}$, et al. Image-guided, targeted and triggered drug delivery to tumors using polymer-based microbubbles. J Control Release. 2012;163:75-81

50. Prinzen L, Miserus RJ, Dirksen A, et al. Optical and magnetic resonance imaging of cell death and platelet activation using annexin a5-functionalized quantum dots. Nano Lett. 2007;7:93-100

51. Miserus RJ, Herias MV, Prinzen L, et al. Molecular mri of early thrombus formation using a bimodal alpha2-antiplasmin-based contrast agent. JACC Cardiovasc Imaging. 2009;2:987-996

52. Wilkinson IB, McEniery CM. Arterial stiffness, endothelial function and novel pharmacological approaches. Clin Exp Pharmacol Physiol. 2004;31:795-799

53. Plenz GA, Deng MC, Robenek H, et al. Vascular collagens: Spotlight on the role of type viii collagen in atherogenesis. Atherosclerosis. 2003;166:1-11 
54. Rodriguez-Feo JA, Sluijter JP, de Kleijn DP, et al. Modulation of collagen turnover in cardiovascular disease. Curr Pharm Des. 2005;11:2501-2514

55. Heeneman S, Cleutjens JP, Faber BC, et al. The dynamic extracellular matrix: Intervention strategies during heart failure and atherosclerosis. J Pathol. 2003;200:516-525

56. Katsuda S, Kaji T. Atherosclerosis and extracellular matrix. J Atheroscler Thromb. 2003;10:267-274

57. Adiguzel E, Ahmad PJ, Franco C, et al. Collagens in the progression and complications of atherosclerosis. Vasc Med. 2009;14:73-89

58. Megens RT, Oude Egbrink MG, Cleutjens JP, et al. Imaging collagen in intact viable healthy and atherosclerotic arteries using fluorescently labeled cna35 and two-photon laser scanning microscopy. Mol Imaging. 2007;6:247-260

59. Megens RT, oude Egbrink MG, Merkx M, et al. Two-photon microscopy on vital carotid arteries: Imaging the relationship between collagen and inflammatory cells in atherosclerotic plaques. J Biomed Opt. 2008;13:044022

60. Zoumi A, Lu X, Kassab GS, et al. Imaging coronary artery microstructure using second-harmonic and two-photon fluorescence microscopy. Biophys J. 2004;87:2778-2786

61. Le TT, Langohr IM, Locker MJ, et al. Label-free molecular imaging of atherosclerotic lesions using multimodal nonlinear optical microscopy. J Biomed Opt. 2007;12:054007

62. Stary $\mathrm{HC}$, Chandler $\mathrm{AB}$, Dinsmore $\mathrm{RE}$, et al. A definition of advanced types of atherosclerotic lesions and a histological classification of atherosclerosis. A report from the committee on vascular lesions of the council on arteriosclerosis, american heart association. Circulation. 1995;92:1355-1374

63. Wyman RA, Mays ME, McBride PE, et al. Ultrasound-detected carotid plaque as a predictor of cardiovascular events. Vasc Med. 2006;11:123-130

64. Magnussen CG, Thomson $\mathrm{R}$, Juonala $\mathrm{M}$, et al. Use of b-mode ultrasound to examine preclinical markers of atherosclerosis: Image quality may bias associations between adiposity and measures of vascular structure and function. J Ultrasound Med. 2011;30:363-369

65. Kaufmann BA, Sanders JM, Davis C, et al. Molecular imaging of inflammation in atherosclerosis with targeted ultrasound detection of vascular cell adhesion molecule-1. Circulation. 2007;116:276-284

66. Nahrendorf $\mathrm{M}$, Keliher $\mathrm{E}$, Panizzi $\mathrm{P}$, et al. $18 \mathrm{f}-4 \mathrm{v}$ for pet-ct imaging of vcam-1 expression in atherosclerosis. JACC Cardiovasc Imaging. 2009;2:1213-1222

67. Rudd JH, Myers KS, Bansilal $\mathrm{S}$, et al. Atherosclerosis inflammation imaging with 18f-fdg pet: Carotid, iliac, and femoral uptake reproducibility, quantification methods, and recommendations. J Nucl Med. 2008;49:871-878

68. Wintermark $\mathrm{M}$, Jawadi SS, Rapp JH, et al. High-resolution ct imaging of carotid artery atherosclerotic plaques. AJNR Am J Neuroradiol. 2008;29:875-882

69. Oppenheim C, Touze E, Leclerc $X$, et al. [high resolution mri of carotid atherosclerosis: Looking beyond the arterial lumen]. J Radiol. 2008;89:293301 
70. Hur J, Park J, Kim YJ, et al. Use of contrast enhancement and high-resolution $3 \mathrm{~d}$ black-blood mri to identify inflammation in atherosclerosis. JACC Cardiovasc Imaging. 2010;3:1127-1135

71. Weber C, Noels H. Atherosclerosis: Current pathogenesis and therapeutic options. Nat Med. 2011;17:1410-1422

72. Szmitko PE, Wang $\mathrm{CH}$, Weisel RD, et al. Biomarkers of vascular disease linking inflammation to endothelial activation: Part ii. Circulation. 2003;108:20412048

73. Ballantyne CM, Abe Y. Molecular markers for atherosclerosis. J Cardiovasc Risk. 1997;4:353-356

74. Tsourkas A, Shinde-Patil VR, Kelly KA, et al. In vivo imaging of activated endothelium using an anti-vcam-1 magnetooptical probe. Bioconjug Chem. 2005;16:576-581

75. Kelly KA, Allport JR, Tsourkas A, et al. Detection of vascular adhesion molecule-1 expression using a novel multimodal nanoparticle. Circ Res. 2005;96:327-336

76. Nahrendorf $M$, Jaffer FA, Kelly KA, et al. Noninvasive vascular cell adhesion molecule-1 imaging identifies inflammatory activation of cells in atherosclerosis. Circulation. 2006;114:1504-1511

77. Kaufmann BA, Carr CL, Belcik JT, et al. Molecular imaging of the initial inflammatory response in atherosclerosis: Implications for early detection of disease. Arterioscler Thromb Vasc Biol. 2010;30:54-59

78. Doring $\mathrm{Y}$, Drechsler $\mathrm{M}$, Wantha $\mathrm{S}$, et al. Lack of neutrophil-derived cramp reduces atherosclerosis in mice. Circ Res. 2012;110:1052-1056

79. Lalloyer F, Fievet C, Lestavel S, et al. The rxr agonist bexarotene improves cholesterol homeostasis and inhibits atherosclerosis progression in a mouse model of mixed dyslipidemia. Arterioscler Thromb Vasc Biol. 2006;26:27312737

80. Haukkala J, Laitinen L, Luoto $\mathrm{P}$, et al. 68 ga-dota-rdg peptide: Biodistribution and binding into atherosclerotic plaques in mice. Eur J Nucl Medl Mol Imaging. 2009;36:2058-2067

81. Sirol M, Aguinaldo JG, Graham PB, et al. Fibrin-targeted contrast agent for improvement of in vivo acute thrombus detection with magnetic resonance imaging. Atherosclerosis. 2005;182:79-85

82. Makowski MR, Forbes SC, Blume $U$, et al. In vivo assessment of intraplaque and endothelial fibrin in apoe(-/-) mice by molecular mri. Atherosclerosis. 2012

83. Aukrust $\mathrm{P}$, Halvorsen B, Ueland $\mathrm{T}$, et al. Activated platelets and atherosclerosis. Expert Rev Cardiovasc Ther. 2010;8:1297-1307

84. Heidt T, Deininger F, Peter $\mathrm{K}$, et al. Activated platelets in carotid artery thrombosis in mice can be selectively targeted with a radiolabeled singlechain antibody. PLoS One. 2011;6:e18446

85. Reitsma S, oude Egbrink MG, Vink H, et al. Endothelial glycocalyx structure in the intact carotid artery: A two-photon laser scanning microscopy study. J Vasc Res. 2011;48:297-306 
86. van Triest HJ, Megens RT, van Assen HC, et al. Arterial radius estimation from microscopic data using a new algorithm for circle parameter estimation. J Biomed Opt. 2010;15:026012

87. Napoli C, Ignarro LJ. Nitric oxide and atherosclerosis. Nitric Oxide. 2001;5:8897

88. Boerboom RA, Krahn KN, Megens RT, et al. High resolution imaging of collagen organisation and synthesis using a versatile collagen specific probe. $J$ Struct Biol. 2007;159:392-399

89. Ashworth SL, Sandoval RM, Tanner GA, et al. Two-photon microscopy: Visualization of kidney dynamics. Kidney Int. 2007;72:416-421

90. Hall AM, Crawford C, Unwin RJ, et al. Multiphoton imaging of the functioning kidney. J Am Soc Nephrol. 2011;22:1297-1304

91. Peti-Peterdi J, Burford JL, Hackl MJ. The first decade of using multiphoton microscopy for high-power kidney imaging. Am J Physiol Renal Physiol. 2012;302:F227-233

92. Sipos A, Toma I, Kang JJ, et al. Advances in renal (patho)physiology using multiphoton microscopy. Kidney Int. 2007;72:1188-1191

93. Provenzano PP, Eliceiri KW, Keely PJ. Multiphoton microscopy and fluorescence lifetime imaging microscopy (flim) to monitor metastasis and the tumor microenvironment. Clin Exp Metastasis. 2009;26:357-370

94. Wyckoff J, Gligorijevic B, Entenberg D, et al. High-resolution multiphoton imaging of tumors in vivo. Cold Spring Harb Protoc. 2011;2011:1167-1184

95. Kiessling F, Razansky D, Alves F. Anatomical and microstructural imaging of angiogenesis. Eur J Nucl Med Mol Imaging. 2010;37 Suppl 1:S4-19

96. Mantopoulos D, Cruzat A, Hamrah P. In vivo imaging of corneal inflammation: New tools for clinical practice and research. Semin Ophthalmol. 2010;25:178185

97. Tsai $\mathrm{TH}$, Jee $\mathrm{SH}$, Dong $\mathrm{CY}$, et al. Multiphoton microscopy in dermatological imaging. J Dermatol Sci. 2009;56:1-8

98. Nava RG, Li W, Gelman AE, et al. Two-photon microscopy in pulmonary research. Semin Immunopathol. 2010;32:297-304

99. Dong J, Revilla-Sanchez R, Moss S, et al. Multiphoton in vivo imaging of amyloid in animal models of alzheimer's disease. Neuropharmacology. 2010;59:268-275

100. Eichhoff $\mathrm{G}$, Busche MA, Garaschuk O. In vivo calcium imaging of the aging and diseased brain. Eur J Nucl Med Mol Imaging. 2008;35 Suppl 1:S99-106

101. Sigler A, Murphy TH. In vivo 2-photon imaging of fine structure in the rodent brain: Before, during, and after stroke. Stroke. 2010;41:S117-123

102. Venneti S, Wiley CA, Kofler J. Imaging microglial activation during neuroinflammation and alzheimer's disease. J Neuroimmune Pharmacol. 2009;4:227-243

103. Kohler A, Geiger H, Gunzer M. Imaging hematopoietic stem cells in the marrow of long bones in vivo. Methods Mol Biol. 2011;750:215-224

104. Coppieters K, Martinic MM, Kiosses WB, et al. A novel technique for the in vivo imaging of autoimmune diabetes development in the pancreas by twophoton microscopy. PLoS One. 2010;5:e15732 
105. Henrickson SE, Mempel TR, Mazo IB, et al. In vivo imaging of t cell priming. Sci Signal. 2008;1:pt2

106. Megens RT, Kemmerich K, Pyta J, et al. Intravital imaging of phagocyte recruitment. Thromb Haemost. 2011;105:802-810

107. Megens RT, Soehnlein O. Seeing is believing: An update on modalities to image inflammation in vivo. Thromb Haemost. 2011;105:774-775

108. Germain RN, Robey EA, Cahalan MD. A decade of imaging cellular motility and interaction dynamics in the immune system. Science. 2012;336:1676-1681

109. Okada T. Two-photon microscopy analysis of leukocyte trafficking and motility. Semin Immunopathol. 2010;32:215-225

110. Garside P, Brewer J. In vivo imaging of infection immunology--4i's! Semin Immunopathol. 2010;32:289-296

111. Santisakultarm TP, Cornelius NR, Nishimura N, et al. In vivo two-photon excited fluorescence microscopy reveals cardiac- and respiration-dependent pulsatile blood flow in cortical blood vessels in mice. Am J Physiol Heart Circ Physiol. 2012;302:H1367-1377

112. Kamoun WS, Chae SS, Lacorre DA, et al. Simultaneous measurement of rbc velocity, flux, hematocrit and shear rate in vascular networks. Nat Methods. 2010;7:655-660

113. Fumagalli S, Coles JA, Ejlerskov $P$, et al. In vivo real-time multiphoton imaging of $\mathrm{t}$ lymphocytes in the mouse brain after experimental stroke. Stroke. 2011;42:1429-1436

114. Millington OR, Brewer JM, Garside $P$, et al. Imaging interactions between the immune and cardiovascular systems in vivo by multiphoton microscopy. Methods Mol Biol. 2010;616:193-206

115. Satoh M, Kobayashi S, Kuwabara A, et al. In vivo visualization of glomerular microcirculation and hyperfiltration in streptozotocin-induced diabetic rats. Microcirculation. 2010;17:103-112

116. Fernandez-Klett F, Offenhauser N, Dirnagl U, et al. Pericytes in capillaries are contractile in vivo, but arterioles mediate functional hyperemia in the mouse brain. Proc Natl Acad Sci U S A. 2010;107:22290-22295

117. Koike $\mathrm{Y}$, Tanaka $\mathrm{K}$, Okugawa $\mathrm{Y}$, et al. In vivo real-time two-photon microscopic imaging of platelet aggregation induced by selective laser irradiation to the endothelium created in the beta-actin-green fluorescent protein transgenic mice. J Thromb Thrombolysis. 2011;32:138-145

118. Dunphy MP, Entenberg D, Toledo-Crow R, et al. In vivo microcartography and subcellular imaging of tumor angiogenesis: A novel platform for translational angiogenesis research. Microvasc Res. 2009;78:51-56

119. Friedl P. Dynamic imaging of cancer invasion and metastasis: Principles and preclinical applications. Clinical \& experimental metastasis. 2009;26:269-271

120. Megens RT, Reitsma S, Prinzen L, et al. In vivo high-resolution structural imaging of large arteries in small rodents using two-photon laser scanning microscopy. J Biomed Opt. 2010;15:011108

121. Yu W, Braz JC, Dutton AM, et al. In vivo imaging of atherosclerotic plaques in apolipoprotein e deficient mice using nonlinear microscopy. J Biomed Opt. 2007;12:054008 
122. Dumont EA, Reutelingsperger CP, Smits JF, et al. Real-time imaging of apoptotic cell-membrane changes at the single-cell level in the beating murine heart. Nat Med. 2001;7:1352-1355

123. Kuhnle GE, Leipfinger FH, Goetz AE. Measurement of microhemodynamics in the ventilated rabbit lung by intravital fluorescence microscopy. J Appl Physiol. 1993;74:1462-1471

124. Kartalis N, Loizou L, Edsborg $\mathrm{N}$, et al. Optimising diffusion-weighted $\mathrm{mr}$ imaging for demonstrating pancreatic cancer: A comparison of respiratorytriggered, free-breathing and breath-hold techniques. Eur Radiol. 2012;22:2186-2192

125. Fan GY, Fujisaki $\mathrm{H}$, Miyawaki $\mathrm{A}$, et al. Video-rate scanning two-photon excitation fluorescence microscopy and ratio imaging with cameleons. Biophys J. 1999;76:2412-2420

126. Niesner RA, Andresen V, Gunzer M. Intravital two-photon microscopy: Focus on speed and time resolved imaging modalities. Immunol Rev. 2008;221:7-25

127. Kurtz R, Fricke $M$, Kalb J, et al. Application of multiline two-photon microscopy to functional in vivo imaging. J Neurosci Methods. 2006;151:276-286

128. Douma K, Megens RT, van Zandvoort MA. Optical molecular imaging of atherosclerosis using nanoparticles: Shedding new light on the darkness. Wiley Interdiscip Rev Nanomed Nanobiotechnol. 2011;3:376-388

129. Rademakers T, Douma K, Hackeng TM, et al. Plaque-associated vasa vasorum in aged apolipoprotein e-deficient mice exhibit proatherogenic functional features in vivo. Arterioscler Thromb Vasc Biol. 2013;33:249-256

130. Li W, Nava RG, Bribriesco AC, et al. Intravital 2-photon imaging of leukocyte trafficking in beating heart. J Clin Invest. 2012;122:2499-2508

131. Lee $\mathrm{S}$, Vinegoni C, Feruglio PF, et al. Real-time in vivo imaging of the beating mouse heart at microscopic resolution. Nat Commun. 2012;3:1054

132. Douma K, Prinzen L, Slaaf DW, et al. Nanoparticles for optical molecular imaging of atherosclerosis. Small. 2009;5:544-557

133. Omura $\mathrm{M}$, Tanaka $\mathrm{A}$, Hirata $\mathrm{M}$, et al. Testicular toxicity of gallium arsenide, indium arsenide, and arsenic oxide in rats by repetitive intratracheal instillation. Fundam Appl Toxicol. 1996;32:72-78

134. Zimmer JP, Kim SW, Ohnishi S, et al. Size series of small indium arsenide-zinc selenide core-shell nanocrystals and their application to in vivo imaging. J Am Chem Soc. 2006;128:2526-2527

135. Fan HM, Olivo M, Shuter B, et al. Quantum dot capped magnetite nanorings as high performance nanoprobe for multiphoton fluorescence and magnetic resonance imaging. J Am Chem Soc. 2010;132:14803-14811

136. Oostendorp M, Douma K, Hackeng TM, et al. Quantitative molecular magnetic resonance imaging of tumor angiogenesis using cngr-labeled paramagnetic quantum dots. Cancer Res. 2008;68:7676-7683

137. Tang S, Zhou Y, Ju MJ. Multimodal optical imaging with multiphoton microscopy and optical coherence tomography. J Biophotonics. 2012;5:396403 
138. Swirski FK, Berger CR, Figueiredo JL, et al. A near-infrared cell tracker reagent for multiscopic in vivo imaging and quantification of leukocyte immune responses. PLoS One. 2007;2:e1075

139. Lippincott-Schwartz J, Patterson GH. Development and use of fluorescent protein markers in living cells. Science. 2003;300:87-91

140. Lukyanov KA, Chudakov DM, Lukyanov S, et al. Innovation: Photoactivatable fluorescent proteins. Nat Rev Mol Cell Biol. 2005;6:885-891

141. Remington SJ. Fluorescent proteins: Maturation, photochemistry and photophysics. Curr Opin Struct Biol. 2006;16:714-721

142. Calfon MA, Rosenthal A, Mallas G, et al. In vivo near infrared fluorescence (nirf) intravascular molecular imaging of inflammatory plaque, a multimodal approach to imaging of atherosclerosis. J Vis Exp. 2011

143. Victora GD, Schwickert TA, Fooksman DR, et al. Germinal center dynamics revealed by multiphoton microscopy with a photoactivatable fluorescent reporter. Cell. 2010;143:592-605

144. Dutta P, Courties G, Wei $\mathrm{Y}$, et al. Myocardial infarction accelerates atherosclerosis. Nature. 2012;487:325-329

145. Phan TG, Bullen A. Practical intravital two-photon microscopy for immunological research: Faster, brighter, deeper. Immunol Cell Biol. 2010;88:438-444

146. Mahou P, Zimmerley M, Loulier K, et al. Multicolor two-photon tissue imaging by wavelength mixing. Nat Methods. 2012;9:815-818

147. Mclnnes E. Artefacts in histopathology. Comparative Clinical Pathology. 2004;13:100-108

148. Sandell JL, Zhu TC. A review of in-vivo optical properties of human tissues and its impact on pdt. J Biophotonics. 2011;4:773-787

149. Cheong WF, Prahl SA, Welch AJ. A review of the optical properties of biological tissues. Journal of Quantum Electronics. 1990;26:2166-2185

150. Piston DW. Imaging living cells and tissues by two-photon excitation microscopy. Trends Cell Biol. 1999;9:66-69

151. Tauer U. Advantages and risks of multiphoton microscopy in physiology. Exp Physiol. 2002;87:709-714

152. Zipfel WR, Williams RM, Webb WW. Nonlinear magic: Multiphoton microscopy in the biosciences. Nature biotechnology. 2003;21:1369-1377

153. Breunig HG, Studier H, Konig K. Multiphoton excitation characteristics of cellular fluorophores of human skin in vivo. Opt Express. 2010;18:7857-7871

154. Koehler MJ, Zimmermann S, Springer S, et al. Keratinocyte morphology of human skin evaluated by in vivo multiphoton laser tomography. Skin Res Technol. 2011;17:479-486

155. Lee $A M$, Wang $H, Y u ~ Y$, et al. In vivo video rate multiphoton microscopy imaging of human skin. Opt Lett. 2011;36:2865-2867

156. Kiesslich R, Burg J, Vieth $\mathrm{M}$, et al. Confocal laser endoscopy for diagnosing intraepithelial neoplasias and colorectal cancer in vivo. Gastroenterology. 2004;127:706-713 
157. Goetz M, Hoffman A, Galle PR, et al. Confocal laser endoscopy: New approach to the early diagnosis of tumors of the esophagus and stomach. Future Oncol. 2006;2:469-476

158. Paramsothy S, Leong RW. Endoscopy: Fluorescein contrast in confocal laser endomicroscopy. Nat Rev Gastroenterol Hepatol. 2010;7:366-368

159. Neurath MF, Kiesslich R. Molecular detection of cd44v6 on aberrant crypt foci by confocal laser endoscopy. Endoscopy. 2010;42 Suppl 2:E314-315

160. Thiberville L, Salaun M, Lachkar S, et al. Confocal fluorescence endomicroscopy of the human airways. Proc Am Thorac Soc. 2009;6:444-449

161. Konig K, Ehlers A, Riemann I, et al. Clinical two-photon microendoscopy. Microsc Res Tech. 2007;70:398-402

162. Rivera DR, Brown CM, Ouzounov DG, et al. Compact and flexible raster scanning multiphoton endoscope capable of imaging unstained tissue. Proc Natl Acad Sci U S A. 2011;108:17598-17603

163. Bortoletto F, Bonoli C, Panizzolo P, et al. Multiphoton fluorescence microscopy with grin objective aberration correction by low order adaptive optics. PLoS One. 2011;6:e22321

164. Kim $\mathrm{P}$, Puoris'haag $\mathrm{M}$, Cote $\mathrm{D}$, et al. In vivo confocal and multiphoton microendoscopy. J Biomed Opt. 2008;13:010501

165. Chen $M, X u C$, Webb WW. Endoscope lens with dual fields of view and resolutions for multiphoton imaging. Opt Lett. 2010;35:2735-2737

166. Pavlova I, Hume KR, Yazinski SA, et al. Multiphoton microscopy as a diagnostic imaging modality for lung cancer. Proc Soc Photo Opt Instrum Eng. 2010;7569:756918 


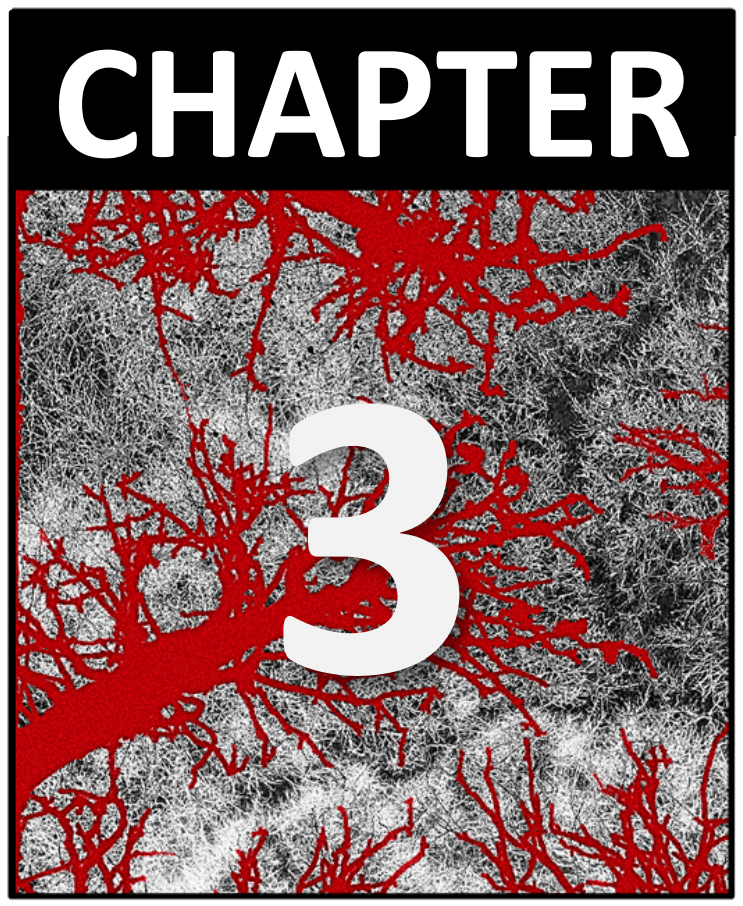




\title{
Plaque-associated vasa vasorum in aged apolipoprotein-E deficient mice exhibit pro-atherogenic functional features in vivo
}

\author{
Timo Rademakers* , Kim Douma*, Tilman M. Hackeng, Mark J. Post, \\ Judith C. Sluimer, Mat J.A.P. Daemen, Erik A.L. Biessen, \\ Sylvia Heeneman, and Marc A.M.J. van Zandvoort \\ * authors contributed equally
}




\section{Abstract}

Neovascularization of human atherosclerotic plaques is implicated in plaque progression and destabilization, although its functional implications are yet unresolved. Here, we aimed to elucidate functional and morphological properties of plaque microvessels in mice in vivo.

Atherosclerotic carotid arteries from aged (>40wks) $\mathrm{ApoE}^{-/-}$mice $(n=51)$ were imaged in vivo using multiphoton laser scanning microscopy. Two distinct groups of vasa vasorum microvessels were observed at sites of atherosclerosis development (median diameters of 18.5 and $5.9 \mu \mathrm{m}$, respectively), while microvessels within the plaque could only rarely be found. In vivo imaging showed ongoing angiogenic activity and injection of FITCdextran confirmed active perfusion. Plaque vasa vasorum showed increased microvascular leakage, combined with a loss of endothelial glycocalyx. Mean blood flow velocity in plaque-associated vasa vasorum was reduced by $\pm 50 \%$ compared to diameter-matched control capillaries, while mean blood flow was reduced 8-fold. Leukocyte adhesion and extravasation were increased 6-fold in vasa vasorum versus control capillaries.

Using a novel in vivo functional imaging strategy, we showed that plaque-associated vasa vasorum were angiogenically active and, albeit poorly, perfused. Moreover, plaque-associated vasa vasorum showed increased permeability, reduced blood flow, and increased leukocyte adhesion and extravasation, i.e. characteristics that could contribute to plaque progression and destabilization. 


\section{Introduction}

Human atherosclerotic plaques are characterized by local expansion of the vasa vasorum and development of intraplaque microvasculature. The extent of vascularization has been shown to correlate with progression stage, inflammatory content of the plaque and plaque instability ${ }^{1,2}$. Ever since Köster suggested that advanced atherosclerotic lesions are associated with an increased density in vasa vasorum ${ }^{3}$, a growing number of studies has shown that both intraplaque microvasculature and vasa vasorum expansion enhance human atherosclerotic plaque progression ${ }^{1,4,5}$. Intraplaque microvessels are thought to be derived mainly from the vasa vasorum by angiogenic sprouting, followed by ingrowth into the lesion ${ }^{6-8}$. The angiogenic response itself is considered to arise from the gradual development of lesional hypoxia due to (I) increased physical distance to the lumen, exceeding the diffusion distance of oxygen, and (II) increased local metabolic activity of the plaque's inflammatory content ${ }^{1,9}$. Additionally, the endothelium of the newly formed microvessels is prone to continuous exposure to inflammatory mediators, which could lead to impaired functionality and increased permeability, caused by deteriorated inter-endothelial junctions and basement membrane detachment ${ }^{10}$. Dysfunctional microvessels would favor influx of circulating erythrocytes, leukocytes and blood platelets into the plaque ${ }^{6,7,11}$, thereby promoting plaque expansion, destabilization and thrombogenicity.

Despite the clinical relevance of plaque neovascularization, experimental animal studies on functionality of plaque microvessels have been hampered by their low incidence, or even lack thereof, in mouse models of atherosclerosis. Pro-angiogenic interventions in murine models have shown to increase plaque development and disease burden ${ }^{12-16}$, whereas antiangiogenic therapy decreases atherosclerosis development ${ }^{17-20}$, implicating a potent role for angiogenesis in experimental atherosclerosis models. However, the interventions also influenced the inflammatory response, thereby affecting lesion development independent of plaque neovascularization. In general, animal studies have failed to show a direct causal relation between plaque vascularization on the one hand, and plaque progression and destabilization on the other.

Lack of adequate detection tools also hampered functional studies of plaque microvessels. Several methods like magnetic resonance imaging ${ }^{21,22}$ or 


\section{CHAPTER 3}

micro- and nano-computed tomography ${ }^{23}$ have been applied to investigate plaque vascularization in murine models of atherosclerosis. These methods lack the spatial and/or temporal resolution to detect functional and structural properties of individual microvessels, or to distinguish between the artery and microvessels. Also, these techniques lack the ability to detect multiple imaging probes simultaneously. Multiphoton Laser Scanning Microscopy (MPLSM) can overcome these shortcomings and has previously been used for in situ imaging of healthy and atherosclerotic arteries at subcellular resolution ${ }^{24,25}$, as well as for quantifying angiogenic activity in models of tumor vascularization and myocardial infarction ${ }^{26,27}$. In addition, MPLSM has been used to image large blood vessels in vivo, triggered on heart rate and respiration to reduce motion artifacts ${ }^{28,29}$. More recently, MPLSM was used to quantify blood flow and haematocrit in blood vessels ${ }^{30}$, substantiating MPLSM as a valuable tool for functional studies of the (micro)vasculature.

Here, we studied plaque microvessels to elucidate their structural and functional characteristics. Using MPLSM imaging in a novel in vivo imaging strategy, plaque microvessels could be visualized in aged $\mathrm{ApoE}^{-/-}$mice. More importantly, angiogenic activity could be visualized and functional characteristics, including blood flow, adhesion and extravasation of leukocytes, and microvascular permeability could be quantified and were found to differ significantly from control microvascular beds. 


\section{Methods}

\section{Animals}

For ex vivo and in vivo experiments aged $\mathrm{ApoE}^{-/-}$mice (40-50 wks of age; $n=51$ ) with advanced atherosclerotic plaques, were used. Young ApoE ${ }^{-/-}$mice (12-16 wks of age; $n=8$ ), without or with only initial atherosclerotic plaques, and agematched wild-type C57BI/6 mice $(n=20)$ served as controls. Mice were supplied by the institutional animal facility and were housed in conventional cages and received a normal chow diet. Experiments were approved by the local animal ethics committee and performed according to institutional guidelines.

For ex vivo MPLSM, mice were anaesthetized by a dorsal subcutaneous injection with ketamine $(100 \mathrm{mg} / \mathrm{kg}$ body weight, Nematek) and xylazine (10 $\mathrm{mg} / \mathrm{kg}$ body weight, Sedamun). Hereafter, animals were injected with the fluorescently labeled ligands via the tail vein and after 10 minutes of circulation of the ligands the animals were sacrificed. The lumen of the vasculature was flushed with $0.9 \% \mathrm{NaCl}$ by injecting the heart, and carotid arteries (including the bifurcation), abdominal aorta, as well as heart, liver, spleen and kidney were excised and kept in cold Hanks Balanced Saline Solution (HBSS, pH 7.4, Invitrogen). Connective tissue and surrounding fat tissue was removed and carotid arteries were mounted in a home-built perfusion chamber, after which a transmural pressure of approximately 80 $\mathrm{mmHg}$ was applied to mimic physiological conditions ${ }^{24,25}$. Abdominal aorta and organs were embedded in 2 weight/volume percentage (w/v\%) agarose gel (Invitrogen). Disposables were purchased from Greiner Bio One.

For in vivo MPLSM, mice were anaesthetized using a dorsal subcutaneous injection with ketamine (100 mg/kg body weight, Nematek) and xylazine (10 mg/kg body weight, Sedamun) through a catheter made from a $28 \mathrm{~mm}$ polyethylene tube fitted with a $30 \mathrm{G}$ needle. Body temperature was kept at $37^{\circ} \mathrm{C}$ by means of a heating pad. Every 30 minutes an additional dose of anaesthetics was administered to maintain anaesthesia. The carotid artery was exposed, fluorescently labelled cells and ligands were administered via tail vein injection, and mice were imaged in vivo. After in vivo imaging, mice were sacrificed and in situ imaging was used for further examination. The number of mice used for the various experiments are indicated in Supplemental Table I. 


\section{Fluorescent probes}

A biotinylated anti-CD31 antibody (BD Pharmingen) was used to detect vascular endothelial cells (ECs). The anti-CD31 antibody was conjugated to either streptavidin-quantum dots (QD) 525 (Invitrogen) or streptavidin-QD585 (Invitrogen), depending on the emission spectrum of the co-staining used. Costainings were performed with different probes. Biotinylated cyclic Asn-Gly-Arg (cNGR) was synthesized as described previously ${ }^{26}$ and was used to target CD13, a marker of angiogenic activity of vascular endothelium. Biotinylated anti-CD105 (R\&D Systems) was used as an alternative marker for activated, angiogenic endothelium ${ }^{31,32}$. Expression of these markers was scored as being absent/low or present. Biotinylated Ephrin-B2 and EphB4 (R\&D systems) antibodies were used to assess the arterial or venous origin of the microvessels in vivo, respectively. Biotinylated antibodies were conjugated with either streptavidin-QD525 or streptavidin-QD585 as indicated.

To examine fluid phase perfusion of vessels as well as microvascular leakage, $100-150 \mu \mathrm{l}$ of $70 \mathrm{kDa}$ or $150 \mathrm{kDa}$ fluorescein isothiocyanate (FITC) labelled dextran ( $1 \mathrm{mg} / \mathrm{ml}$; Sigma) was injected into the tail vein to label blood plasma. Imaging was started directly after injection of FITC-dextran and was performed up to 60 minutes. In relation to measuring microvascular leakage, $100 \mu$ FITC-labelled wheat germ agglutinin (WGA, 1mg/ml; Sigma) was injected to assess the status of the glycocalyx in microvessels.

For studying blood flow and blood flow velocity, platelets were isolated from donor $\mathrm{C} 57 \mathrm{BI} / 6$ mice and labelled with carboxyfluorescein $\mathrm{N}$-succinimidyl ester (CFSE, Invitrogen; $5 \mu \mathrm{g} / \mathrm{ml}$ ) as described previously ${ }^{33}$. Briefly, blood from a donor mouse was collected on 0.5 volume parts $5 \mathrm{mM}$ Hepes buffer $\mathrm{pH} 7.14$ containing $20 \mathrm{U} / \mathrm{ml}$ heparin, and platelet rich plasma (PRP) was prepared by centrifugation at 2,000g for 10 minutes PRP was incubated for 15 minutes at room temperature with CFSE. Platelets were then washed and the pellet was resuspended in phosphate-buffered saline (PBS) and platelet concentration was adjusted to the final concentration. In total, $200 \mu \mathrm{l}$ of $5.0 \times 10^{7}$ platelets $/ \mathrm{ml}$ was injected in the tail vein.

Additionally, bone marrow derived cells were isolated from the tibia and femur from $\mathrm{C} 57 \mathrm{BI} / 6$ control mice, incubated with $1 \mu \mathrm{M}$ cell tracker red (CMTPX, Invitrogen) for $30 \mathrm{~min}$ at room temperature, washed and $1.0 \times 10^{7}$ cells were injected into the tail vein. 
For in vivo labeling of leukocytes, $20 \mu \mathrm{l}$ of a Rhodamine $6 \mathrm{G}$ solution (Sigma, $1 \mathrm{mg} / \mathrm{ml}$ in $0.9 \% \mathrm{NaCl}$ solution) was injected into the tail vein as described previously ${ }^{34}$. Imaging was performed directly after injection of Rhodamine $6 \mathrm{G}$ up to 60 minutes to reduce visualization of non-specific binding of Rhodamine $6 \mathrm{G}$ to e.g. endothelial cells.

To better assess intraplaque microvascular structures in ex vivo experiments, vessels were excised and mounted as described above, before incubation with Nile Red ( $500 \mu \mathrm{g} / \mathrm{mL}$ in HBSS buffer) to stain lipids within the plaque. Additionally, nuclear staining was performed in vivo and ex vivo using $2 \mu \mathrm{M}$ Syto41 (Invitrogen) for both ex vivo and in vivo imaging.

\section{Multiphoton laser scanning microscopy}

MPLSM imaging was performed on a Leica SP5 imaging platform (Leica Microsystems, Germany) that integrates multiphoton microscopy with fast resonant scanning, and uses a Compact Ultrafast Ti:Sapphire Laser (Chameleon, Coherent, USA). An excitation wavelength of $880 \mathrm{~nm}$ was used in all experiments. Tissues were observed using a 20x 1.0 water immersion objective (HCX PL APO L, Leica Microsystems, Germany) with a numerical aperture of 1.00 and integrated optical zoom allowing magnification up to 60x. Photo-multiplier tubes were used to detect three spectral regions: $420-470 \mathrm{~nm}$ (Syto41, Second Harmonic Generation (SHG)), 515-560 nm (FITC, CFSE, QD525) and 570-600 nm (QD585, CMTPX, Rhodamin 6G, Nile Red).

Ex vivo image acquisition was performed at a frame size of $512 \times 512$ pixels (pixel size: $1.44 \times 1.44 \mu \mathrm{m}$ ), scanning at $100 \mathrm{~Hz}$ and a frame average filter of 2 was used to reduce noise. In-depth scans were performed at $200 \mathrm{~Hz}$ with a frame average of 2 . Consecutive images had an interplanar distance of $1.00 \mu \mathrm{m}$.

In vivo MPLSM imaging was performed at a frame size of $400 \times 400$ pixels (pixel size: $1.0882 \times 1.0882 \mu \mathrm{m}$ ), scanning at $8000 \mathrm{~Hz}$ for fast recording (20 frames/sec) and using a line average of 2 (10 frames/sec) for more detailed scans. Per time series, a total of 500 frames was recorded. After in vivo imaging, the animal was sacrificed and additional in situ imaging was performed to examine the vessel at higher resolution and an increased signalto-noise ratio without motion artifacts. In situ imaging was performed at a frame size of $512 \times 512$ pixels (pixel size $0.847 \times 0.847 \mu \mathrm{m}$ ), scanning at $250 \mathrm{~Hz}$ and an interplanar distance of $0.50 \mu \mathrm{m}$. 


\section{Data analysis}

All data analyses, except for microvascular permeability analysis, were performed using Image Pro 6.3 (MediaCybernetics). Prior to analysis, all data were deconvolved using AutoQuant X2-AutoDeblur (MediaCybernetics) in 3D blind deconvolution mode. Microvascular permeability was assessed using ImageJ (http://imagej.nih.gov/ij/). 3D rendering was performed using an ImageJ plugin for high level 3D visualization ${ }^{35}$. The analyzed data sets are specified in Supplemental Table II.

Microvessel diameter was assessed by measuring the maximum distance between the opposite sides of the microvessel wall at minimally ten separate points per microvessel, from which the median diameter per microvessel was calculated. Microvessel diameter was determined for intraplaque and adventitial vessels.

Microvascular permeability was determined by calculating the ratio of the extraluminal/intraluminal fluorescence intensity of the FITC-dextrans, and corrected for extra- and intraluminal baseline autofluorescence present before dextran injection. Extraluminal tissue was defined as the area within $1.5 x$ vessel diameter's distance of the microvessel wall. The mean ratio was calculated per time point and plotted.

Adhesion of leukocytes to the vessel wall was assessed by tracking ex vivo (CMTPX) or in vivo (Rhodamin 6G) labeled cells that remained at the same point within the vessels for at least 250 frames (i.e. approximately $30 \mathrm{sec}$; Supplemental Figure I). Adhesion rates were normalized to the microvessel surface, which was derived from the microvessel length and microvessel diameter. The adjacent sternohyoid muscle capillaries and cremaster muscle capillaries served as control microvessels.

Blood flow velocity was derived from in vivo measurements using both ex vivo and in vivo labeled platelets and leukocytes. Movement of single platelets or leukocytes was tracked in microvessels during multiple frames and average travelled distance per 6 frames $(\Delta t=0.357 \mathrm{sec})$ was calculated (Supplemental Figure II). Adherent leukocytes were excluded for blood flow velocity measurements. Flow speed was then compared to control capillaries (sternohyoid muscle capillaries) of similar diameter. Additionally, blood flow velocity was determined in the carotid artery, proximal to the plaque, to validate the blood flow velocity measurements in the microvessels. For determining blood flow velocity in the carotid artery, we used the 
displacement i.e. track length of single platelets and leukocytes within a single frame (Supplemental Figure II-A). Briefly, velocities in both $\mathrm{x}$ and $\mathrm{y}$ direction were determined separately by measuring the displacement in both directions in relation to the respective scanning time interval to calculate the total velocity of the platelet/leukocyte. Velocities were corrected for the angle between the vessel and the imaging plane (Supplemental Figure II-B).

\section{Histology, immunohistochemistry and electron microscopy}

After MPLSM, tissue was harvested, fixed in 1\% paraformaldehyde (PFA) and embedded in paraffin. From all tissues, $4 \mu \mathrm{m}$ sections were cut and stained with haematoxylin-eosin (HE) to assess general morphology and plaque stage. Immunohistochemical stainings for CD31 (Invitrogen) and von Willebrand factor (vWf; DAKO) were performed to stain microvessels in the adventitia surrounding the atherosclerotic lesion.

Also, carotid artery samples were collected on $2.5 \%$ glutaraldehyde (GTA; Ted Pella, Redding, CA, USA) in 0.1M phosphate buffer ( $\mathrm{pH}=7.6)$. After several washes, tissue fragments were post fixed in $1 \%$ osmium tetroxide solution, and routinely dehydrated through 100\% ethanol, cleared with propylene oxide, and embedded in epoxy resin. Ultra-thin sections (70-90 nm) were cut on an ultra-microtome, mounted on Formvar (1595 E, Merck)-coated 75mesh copper grids, and counterstained with uranyl acetate and lead citrate before analysis on a Philips CM100 transmission electron microscope. In total, lesions from 6 aged $\mathrm{ApoE}^{-/-}$mice were evaluated, with 2-5 microvessels examined per lesion.

\section{Statistics}

Data are presented as mean \pm SEM, unless stated otherwise. Statistics were performed using Graphpad Prism 5.0 or PASW Statistics 18. The nonparametric Mann-Whitney U-test was applied to adhesion, extravasation and blood flow velocity data. Microvascular permeability data were analyzed using curve fitting regression analysis. Fisher's exact test was used for examining differences between groups for presence of plaque-associated microvasculature. Results were considered statistically different when $p<0.05$. 


\section{Results}

\section{Vasa vasorum were visualized and morphologically characterized in vivo}

The vasa vasorum were studied in detail using in vivo and in situ MPLSM after labeling with anti-CD31, allowing characterization of microvessel morphology throughout the adventitia. Characteristics like microvessel diameter were similar in the adventitia of the carotid artery and abdominal aorta (Table 1). Based on size and orientation, two types of plaque-specific microvessels could be discriminated. The larger vasa vasorum microvessels (median diameter: $18.5 \mu \mathrm{m}$ ) generally ran in parallel to the artery, whereas smaller microvessels (median diameter: $5.9 \mu \mathrm{m}$ ) mostly covered the artery radially. The adventitia surrounding the atherosclerotic plaque showed several microvessels (Figure $1 \mathrm{~A}$ and 1B, Supplemental Video I and II), which was confirmed by immunohistochemistry for CD31 and von Willebrand factor (vWf) (Supplemental Figure III). Microvessels in peri-adventitial fat were excluded from analysis based on collagen imaging of the adventitia using second harmonic generation (SHG) and imaging of autofluorescence of the periadventitial fat. Microvessels were mostly situated within 40-80 $\mu \mathrm{m}$ from the outer elastic lamina, but occasionally - at sites of large advanced plaques microvessels would be situated within 15-20 $\mu \mathrm{m}$ from the outer elastic lamina.

Table 1. Quantification of microvessel diameter from plaque-associated microvessels in the carotid artery and abdominal aorta; ND: not determined

\begin{tabular}{llccc} 
& Location & Median $(\mu \mathrm{m})$ & Range $(\mu \mathrm{m})$ & $\begin{array}{c}\text { \# of microvessels } \\
\text { (\# mice) }\end{array}$ \\
\hline Carotid artery & Intraplaque & 6.65 & $4.42-9.02$ & $6(20)$ \\
& Adventitial $($ large; $>10 \mu \mathrm{m})$ & 18.5 & $10.5-21.2$ & $71(39)$ \\
& Adventitial $($ small; $<10 \mu \mathrm{m})$ & 5.9 & $5.56-6.23$ & $56(39)$ \\
\hline \multirow{2}{*}{ Abdominal aorta } & Intraplaque & ND & ND & ND \\
& Adventitial $($ large; $>10 \mu \mathrm{m})$ & 22.7 & $16.6-43.8$ & $8(8)$ \\
& Adventitial $($ small; $<10 \mu \mathrm{m})$ & 6.0 & $2.1-16.5$ & $5(8)$
\end{tabular}


Vasa vasorum were exclusively located at atherosclerotic foci around the carotid bifurcation, and were neither observed further upstream at the nondiseased common carotid artery, nor in younger $\mathrm{ApoE}^{-/}$mice or age-matched C57BI/6 controls (Table 2). Individual ECs (Figure 1C, arrowheads) of the vasa vasorum and their nuclei (Figure $1 \mathrm{C}$, arrows) could be delineated.
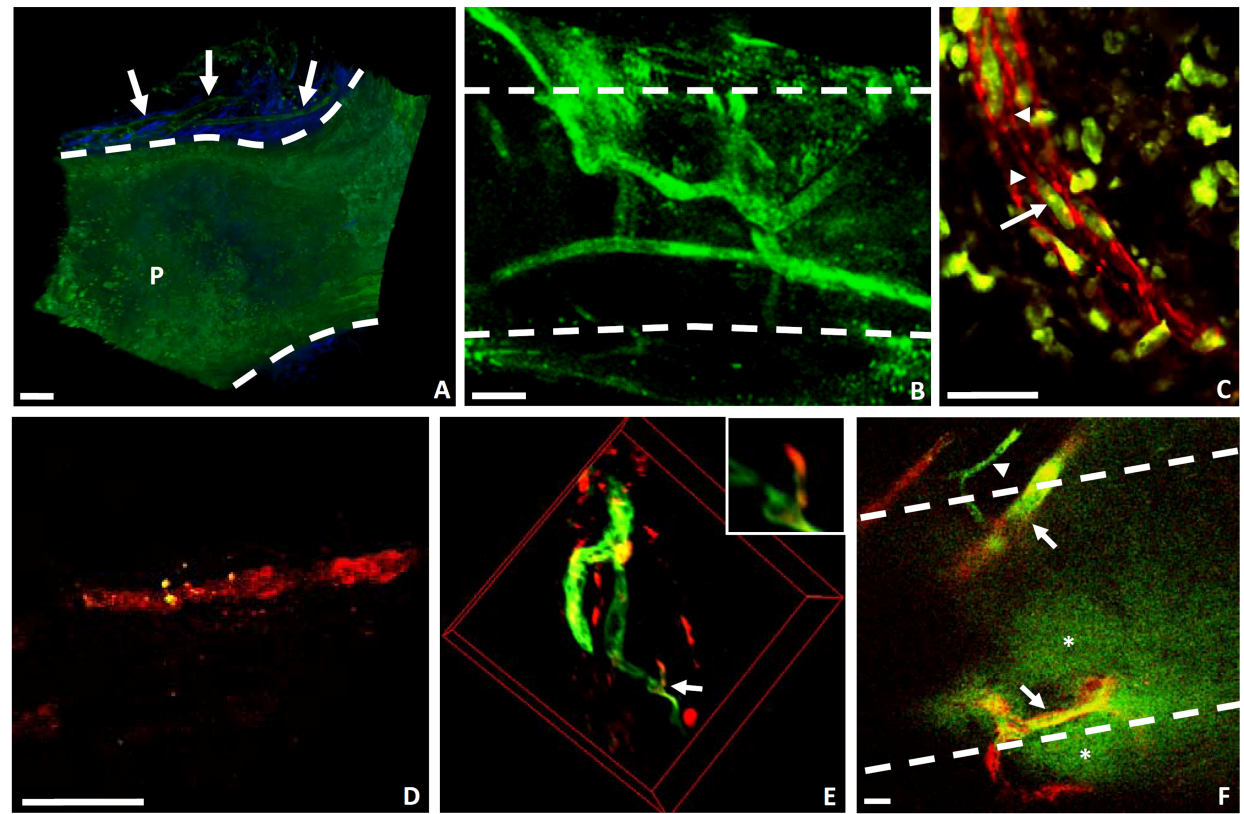

Figure 1. Plaque-associated vasa vasorum of atherosclerotic plaques at the carotid artery bifurcation in aged ApoE ${ }^{-/}$mice could be detected using MPLSM. 3D rendering of the carotid artery directly proximal from the bifurcation and viewed from the luminal side depicts vasa vasorum (green, arrows) embedded in adventitial collagen (blue). Plaque autofluorescence in green $(P)$ is also visible ( $A$, Supplemental Video 1). Also, when focusing merely on the adventitia, the vasa vasorum is clearly detectable (B, green; Supplemental Video 2 ). In $C$, single $C D 31^{+}$(red, arrowheads) endothelial cells including nuclei (green, arrow) were detected. When assessing arterial/venous lineage of the vasa vasorum, microvessels were found to be predominantly arterial in origin as shown by Ephrin-B2 staining (D, red), while the venous marker EphB4 (green) was not detectable. Using a cNGR probe (red, inset figure E) to detect angiogenic endothelium, angiogenic sprouting from a pre-existing vasa vasorum vessel (green) was detected. $C D 31^{+}$vasa vasorum vessels (red, arrows), as well as new sprouts (CD31 ${ }^{\text {low }}$, arrowhead), are actively perfused as determined by a FITC-labeled (green) $70 \mathrm{kDa}$ dextran (F). Leakage of dextran into tissue surrounding the vasa vasorum could be detected $(F, *)$. Dotted lines indicate the outline of the carotid artery, which is situated below the vasa vasorum. Bars indicate $100 \mu \mathrm{m}$ ( $A$ and B) or $25 \mu \mathrm{m}(\mathrm{C}-\mathrm{F})$. 


\section{CHAPTER 3}

Table 2. Prevalence of vasa vasorum in the carotid artery in the various experimental groups

Adventitial microvessels

\begin{tabular}{l|cccc} 
Genotype & Number of mice & No & Yes & \% present \\
\hline C57Bl/6 & 20 & 20 & 0 & $0 * * *$ \\
ApoE $^{-/}(<16$ wks $)$ & 8 & 7 & 1 & $12.5^{* * *}$ \\
ApoE $^{-/}(>40$ wks $)$ & 51 & 6 & 45 & 88.2 \\
$* * * p<0.005 ;$ Fisher's exact test; vs. aged ApoE & & &
\end{tabular}

In vivo measurements displayed a predominantly arterial phenotype of the vasa vasorum (Figure 1D), as observed by Ephrin-B2 and EphB4 staining. Moreover, upon local or bilateral ligation of the jugular vein for up to 30 minutes, functional parameters, e.g. microvessel diameter, remained unaltered (Supplemental Figure IV), arguing a marginal role of the venous system in controlling the vasa vasorum.

\section{Plaque-associated vasa vasorum showed angiogenic activity in vivo}

The particular localization of the microvasculature at sites of plaque formation and absence of microvasculature in young atherosclerotic mice, suggests angiogenesis to underlie the development of these microvessels. Therefore, a cNGR probe targeting the angiogenic marker CD13 and specific for angiogenic endothelium upon in vivo injection ${ }^{26}$ was used to investigate whether ongoing angiogenesis was present. The vasa vasorum showed $\mathrm{CD} 31^{+} / \mathrm{CD} 13^{-}$ microvessels accompanied by occasional $\mathrm{CD} 31^{\text {low }} / \mathrm{CD} 13^{+}$sprouts (Figure $1 \mathrm{E}$ ). At the site of sprouting, part of the endothelium expressed both CD31 and CD13 (Figure 1E, inset). 


\section{Plaque-associated vasa vasorum were actively perfused and hyperpermeable}

As the normal physiology and perfusion of vasa vasorum microvessels were unknown, this was assessed by injection of $70 \mathrm{kDa}$ and $150 \mathrm{kDa}$ FITC-dextrans, which indicated active perfusion (Figure $1 \mathrm{~F}$, green) of the vasa vasorum (red: CD31) in vivo. Also, sprouting of new microvessels was observed (Figure 1F, arrowhead), with new sprouts displaying low CD31 expression. Additionally, a rise in fluorescence intensity, i.e. dextran concentration, in the adventitia was observed (Figure 1F, asterisk).
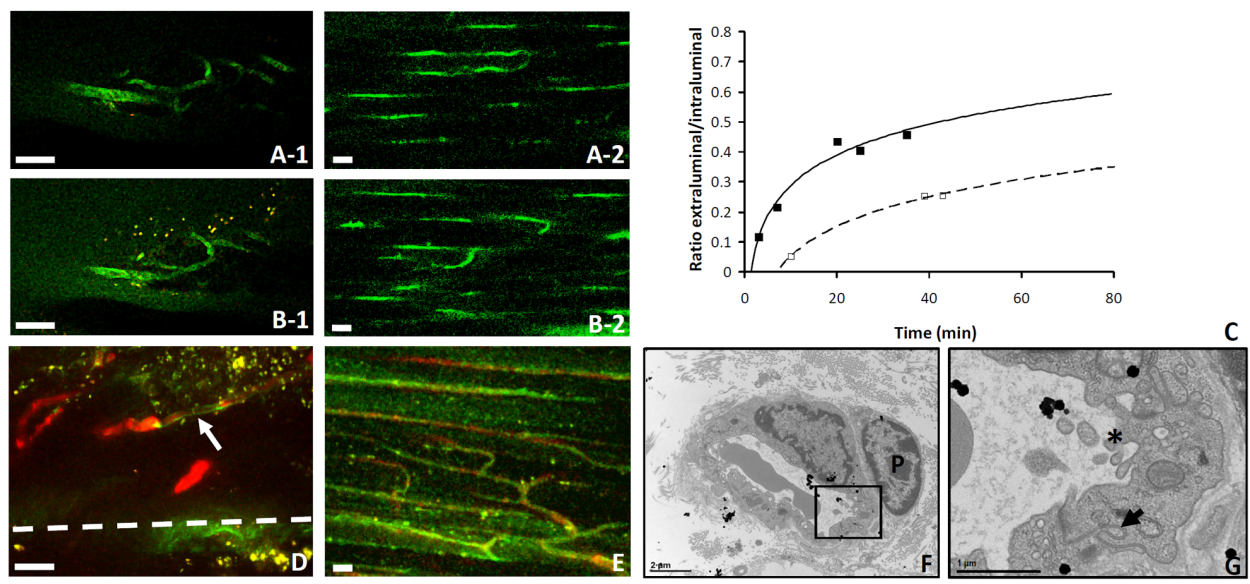

Figure 2. Microvascular permeability was calculated from the ratio of the extraluminal/intraluminal fluorescence intensity of $70 \mathrm{kDa}$ FITC-dextran. Extraluminal fluorescence intensity increased over time, as depicted for $t=3$ and $t=35$ minutes after injection (A-1 and B-1, respectively) for vasa vasorum, while only a limited increase in fluorescence intensity was observed for the control sternohyoid muscle at the same time points (A-2 and B-2, respectively). The ratio extraluminal/intraluminal fluorescence intensity was calculated for different time points, as shown in a representative plot (C). Over time, the extraluminal presence of $70 \mathrm{kDa}$ FITC-dextran increased significantly in the vasa vasorum (black line), but not in control microvessels (dotted line). No significant microvascular leakage was observed for 150 kDa dextrans in either vessel types (not shown). In plaque-associated vasa vasorum (D), the glycocalyx (green: WGA) was present in only a small part of the microvessel (arrow; red: CD31). In contrast, the glycocalyx was consistently present in control microvessels in the sternohyoid muscle (E). Inter-endothelial junctions in plaque-associated vasa vasorum were however intact ( $F$, higher magnification in $G$; arrows), though the endothelial cells did show signs of membrane blebbing $\left(^{*}\right)$. Pericytes $(P)$ surrounding the endothelial cells were also found. Dotted line indicates the outline of the carotid artery, which is situated below the vasa vasorum; bars indicate $25 \mu \mathrm{m}$ $(A-E), 2 \mu m(F)$, and $1 \mu m(G)$. 
Concurrently, dextran injection allowed measurement of microvascular permeability by calculating the time-dependent rise in extraluminal fluorescence intensity compared to intraluminal fluorescence intensity. While intraluminal dextran levels remained constant for over 60 minutes, the extraluminal content of $70 \mathrm{kDa}$ dextran increased with time to an extraluminal/intraluminal ratio of approximately 0.46 after 35 minutes in plaque-associated vasa vasorum (Figure $2 \mathrm{~A}-1$ and $2 \mathrm{~B}-1$ ). Since vasa vasorum, in accordance with literature ${ }^{19,36}$, was not observed in non-diseased arteries, we used the similar sized microvasculature of the nearby sternohyoid muscle as a control. In the sternohyoid muscle, only a limited increase in extravascular dextran concentration was observed (Figure 2A-2 and 2B-2). Upon quantification, extravascular dextran concentration (Figure $2 \mathrm{C}$, black line, $\mathrm{p}<0.05$ ) was significantly increased only in vasa vasorum, but not in control microvessels (Figure 2C, dotted line), indicative of enhanced microvascular permeability in the vasa vasorum. Additionally, extravasation of $70 \mathrm{kDa}$ dextran occurred earlier in vasa vasorum compared to control microvessels (Figure 2C). Due to myocyte autofluorescence, baseline extra-/intraluminal fluorescence ratios varied slightly, however all experiments showed an identical pattern of significantly increased microvascular permeability of the vasa vasorum $(p<0.05)$. Of note, microvascular extravasation of $150 \mathrm{kDa}$ dextran did not significantly increase over time, neither in the vasa vasorum nor in control microvessels (not shown).

A size-dependent increase in permeability could be caused by several mechanisms. It is known that reduction or absence of endothelial glycocalyx in microvessels can, independently from the inter-endothelial junctions, lead to increased permeability and leukocyte adhesion ${ }^{37}$. In addition, disruption of the endothelial barrier may cause hyperpermeability. To determine what caused the increased permeability, we examined glycocalyx integrity by Wheat Germ Agglutinin (WGA) staining and analyzed the integrity of the inter-endothelial junctions by electron microscopy. Staining for WGA in vivo showed an irregular staining pattern in the vasa vasorum (Figure 2D), contrary to an evenly distributed expression of glycocalyx throughout control microvessels in the sternohyoid muscle (Figure 2E; Supplemental Figure V). 

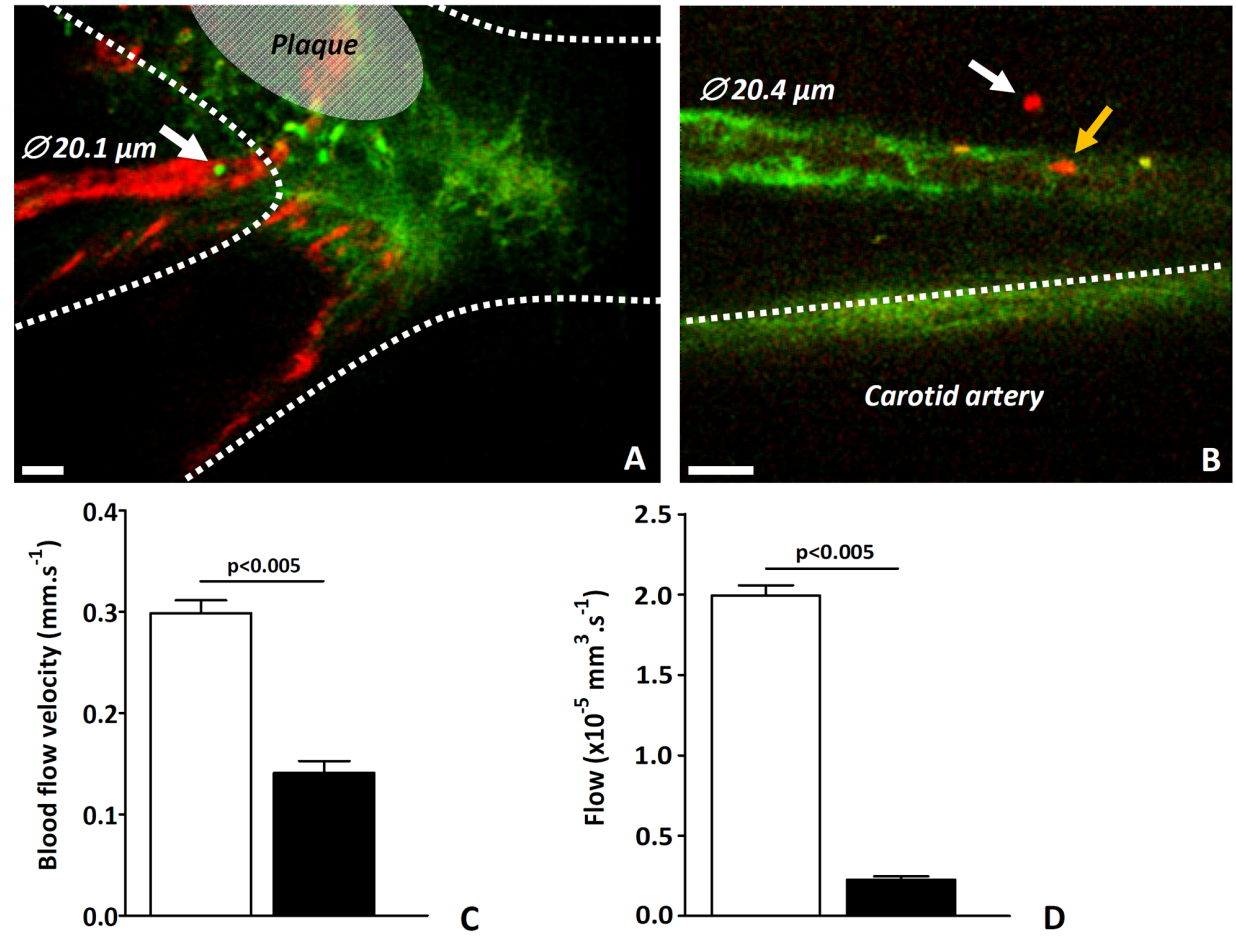

Figure 3. Cellular perfusion of vasa vasorum in aged ApoE ${ }^{-1-}$ mice. In A, vascular endothelium (red), cellular perfusion (green; arrow) and elastin autofluorescence (green) of the carotid artery is also visible. Leukocytes ( $B$; red) adhered to (yellow arrow) or extravasated from (white arrow) the vasa vasorum ( $B$; green). Dotted lines indicate the outline of the carotid artery, situated below the vasa vasorum; bars indicate $25 \mu \mathrm{m}$. Leukocyte velocity in vasa vasorum (black bars, C) is significantly reduced compared to control microvessels (white bars, C). Blood flow in vasa vasorum is also significantly lower than in control microvessels $(D)$.

The inter-endothelial junctions were intact as shown by EM (Figure $2 \mathrm{~F}$ and 2G; arrows), and occasionally, pericytes (P) were found covering the microvascular endothelium. The ECs did however show initial signs of membrane blebbing, indicative of early endothelial dysfunction.

Plaque-associated vasa vasorum showed decreased blood flow, yet an increased adhesion and extravasation of leukocytes

Leukocytes were observed within the vasa vasorum after in vitro (Figure 3A) and in vivo (Figure $3 \mathrm{~B}$ ) labeling. Using labeled leukocytes and platelets, blood flow velocity and blood flow were assessed in the carotid artery, vasa vasorum and control microvessels. Both blood flow velocity (2.1 fold decrease, p $<0.001$; Figure 3C, Supplemental Table I) and blood flow (7.6-9.8 fold 


\section{CHAPTER 3}

decrease, $p<0.001$; Figure 3D) were significantly reduced in vasa vasorum compared to control microvessels. Changes in blood flow and flow velocity were independent of microvessel diameter. Blood flow direction was identical to the main artery.
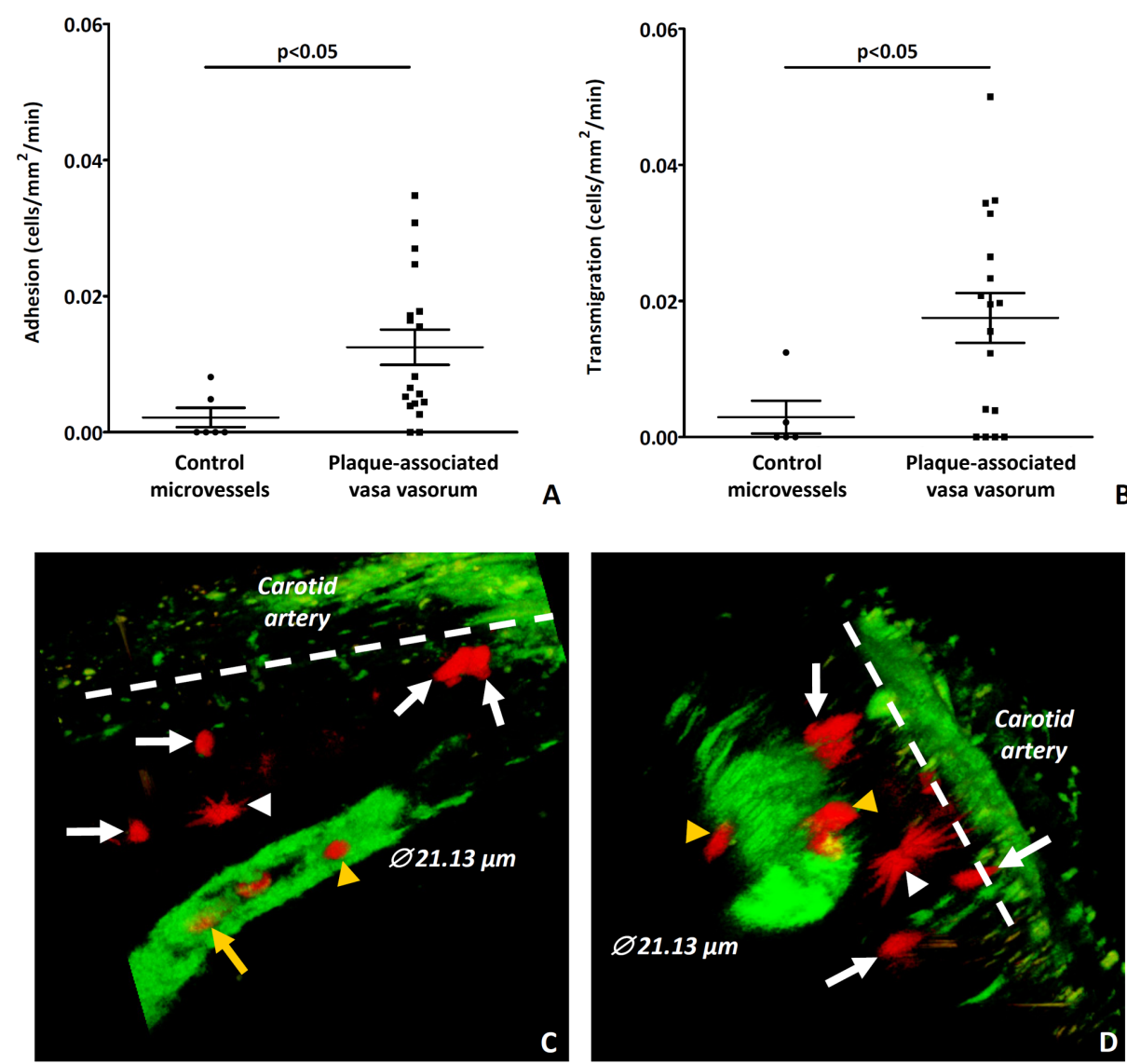

Figure 4. In aged ApoE $^{-/}$mice, adhesion and extravasation was significantly increased in vasa vasorum compared to control microvessels of similar diameters (A and B). This is accompanied by decreased blood flow (see Figure $3 C$ and $3 D$ ). $3 D$ reconstruction (C and $D$ ) of in situ z-scan showed adhering (yellow arrow) and transmigrated cells (white arrows), as well as cells in the progress of transmigration through the microvessel wall (yellow arrowheads). MPLSM allowed differentiation of morphologically distinct cell types, e.g. pseudopodia-rich cells resembling a dendritic morphology (white arrowheads). Dotted line indicates arterial vessel wall at the site of the plaque, bars indicate $25 \mu \mathrm{m}$. 
Additionally, adhesion and transmigration of ex vivo and in vivo labeled leukocytes were significantly increased compared to control microvessels (Figure 4). Increased adhesion (Figure $4 \mathrm{~A}, 1.3 \times 10^{-2} \pm 0.3 \times 10^{-2}$ vs. $0.2 \times 10^{-2} \pm$ $0.1 \times 10^{-2}$ cells $/ \mathrm{mm}^{2} / \mathrm{min}, \mathrm{p}<0.05$ ) and extravasation (Figure $4 \mathrm{~B}, 1.8 \times 10^{-2} \pm$ $0.4 \times 10^{-2}$ vs. $0.3 \times 10^{-2} \pm 0.2 \times 10^{-2}$ cells $/ \mathrm{mm}^{2} / \mathrm{min}, \mathrm{p}<0.05$ ) of leukocytes was observed in plaque-associated vasa vasorum and control microvessels, respectively. Leukocyte adhesion and extravasation was examined in more detail ex vivo, using 3D image analysis. Cell morphology suggested transmigration of different leukocyte subtypes, including pseudopodia-rich cells resembling a dendritic cell morphology, from the vasa vasorum towards the plaque (Figure 4C and 4D, Supplemental Video III).

\section{Ex vivo MPLSM confirmed presence of vasa vasorum and intraplaque microvascular structures}

Plaque-associated vasa vasorum were also imaged ex vivo, allowing imaging at higher resolution and signal-to-noise ratio without motion artifacts. For ex vivo imaging, the carotid artery or abdominal aorta was excised, mounted in a perfusion chamber and imaged under a transmural pressure of $80 \mathrm{mmHg}$ to mimic physiological conditions ${ }^{24}$. Ex vivo imaging was performed up to $30 \mu \mathrm{m}$ from the outer elastic lamina. Vasa vasorum were detected both in the carotid artery and abdominal aorta (Table 1).

Ex vivo imaging clearly visualized intraplaque microvascular structures (Supplemental Figure VI-A and VI-B), yet intraplaque microvessels were only found in 6 out of 20 plaques (Supplemental Table III). Surprisingly, none of these microvessels derived from the vasa vasorum. Rather, data suggested that these microvessels originated from endothelial sprouting from the luminal endothelial layer ( 3 out of 6 plaques; Supplemental Figure VI-C). No functional characteristics could be determined for intraplaque vessels, arguing a limited role for intraplaque microvessels in this model. Also, occurrence of intraplaque hemorrhage (IPH) was determined in aged $\mathrm{ApoE}^{-/}$, and was found in 4 out of $20(20 \%)$ mice examined. Although all mice which showed IPH, also showed vasa vasorum, only a minor fraction of vasa vasorum containing plaques showed features of IPH. Occurrence of IPH could not be linked to occurrence of luminal microvascular structures. 


\section{Discussion}

The presence and role of plaque-associated microvessels in murine plaques has been extensively debated in literature. Previous attempts to detect these microvessels yielded varying results, in part depending on the animal model and visualization techniques used. Here, we used in vivo MPLSM to show that plaque-associated vasa vasorum (I) are present in $\mathrm{ApoE}^{-/-}$mice; (II) show angiogenic activity; (III) are actively perfused and hyperpermeable; (IV) have significantly reduced blood flow and blood flow velocity compared to control microvessels with similar diameter, and (V) show enhanced leukocyte adhesion and transmigration at the site of the atherosclerotic plaque. Taken together, we show that plaque-associated microvessels exhibit functional features which could facilitate plaque development and progression.

The increased adhesion of leukocytes may, in part, be a consequence of the reduction in blood flow velocity, since this would cause a reduction in local shear stress at the microvascular wall. Consequently, low shear stress is deemed to cause endothelial cell dysfunction and upregulation of adhesion molecules like VCAM-1 and ICAM-1 ${ }^{38-40}$. The increased leukocyte adhesion was accompanied by augmented transmigration to the adventitial tissue, facilitating a second site of ingress of leukocytes, besides migration through the luminal endothelium. These cells may contribute to further plaque development, either by functioning in the adventitia itself, or by infiltration into the plaque after migrating through the media. As such, the vasa vasorum could contribute to exacerbation of atherosclerosis, by infiltration of new inflammatory cells ${ }^{41}$.

Additionally, vasa vasorum hyperpermeability may be another important contributor to plaque progression, as it favors extravasation of lipids and plasma proteins at sites of plaque development ${ }^{42}$. Increased permeability may be attributed to disruption of the endothelial barrier function ${ }^{10}$. Our finding of augmented microvascular extravasation in the vasa vasorum, at least for molecules with a molecular weight of up to $70 \mathrm{kDa}$ suggests that abundant plasma proteins, such as albumin, can leak into the adventitia. Indeed, extravascular albumin has been detected in both human and mouse atherosclerotic lesions ${ }^{43,44}$. Here, we extend these findings, demonstrating an apparent size limit for endothelial leakage in vasa vasorum of $70-150 \mathrm{kDa}$ in vivo in aged $\mathrm{ApoE}^{-/-}$mice. The origin of the microvascular hyperpermeability 
remains to be further elucidated. EM did not show any aberrations in endothelial cell junctions, concordant with a recent study from Eriksson ${ }^{36}$. However, the endothelial glycocalyx of plaque-associated vasa vasorum was disrupted, indicating a possible cause for the increase in vascular permeability, and more specifically, permeability for smaller molecules. Both the increased leukocyte extravasation and hyperpermeability will affect plaque development and progression, especially when plaques become more advanced and vasa vasorum has expanded by increasing adventitial inflammation. As such, these processes could enhance plaque progression independent of microvascular disruption and hemorrhage.

Vasa vasorum were abundantly present at sites of atherosclerotic plaques, sites at which we also observed an increase in vessel wall thickness (not shown). Thickening of the vessel wall may infer an increase in vascularization, since oxygenation of the entire tissue is hindered by vessel wall thickening, potentially increasing local hypoxia. Remarkably, although the entire carotid artery was examined, we did not observe any vascularization of non-atherosclerotic segments, neither in control animals nor in the young or aged $\mathrm{ApoE}^{-/-}$mice. This is consistent with reports by e.g. Gräbner et al ${ }^{45}$ and Eriksson ${ }^{36}$. Given the fact that vasa vasorum was absent in non-atherosclerotic segments, we chose the sternohyoid microvasculature as a substitute model for general microvascular blood flow. Taken together, development of vasa vasorum in $\mathrm{ApoE}^{-/-}$mice appears to be an adaptive response to pathological, atherosclerosis-induced vessel wall changes.

Many studies have suggested a role for angiogenesis in atherosclerosis. Pro-atherogenic effects of increased angiogenesis and anti-atherogenic effects upon decreased angiogenesis have been described in models of pharmacological stimulation or inhibition of angiogenesis ${ }^{18,19,46}$, as well as in certain animal models, e.g. the collagen-XVIII/ApoE double knock-out mouse ${ }^{15}$. Here, presence of $\mathrm{CD}_{13} 3^{+} / \mathrm{CD} 31^{\text {low }}$ sprouts in the adventitia substantiates the presence of ongoing angiogenesis. The reduced expression of CD31 is in accordance with studies which have shown that both embryonic stem cells and mature ECs show reduced expression of CD31 during endothelial differentiation, migration and proliferation ${ }^{47,48}$. It is however surprising that the plaque-associated vasa vasorum showed an almost exclusively arterial phenotype, which is in contrast to the model suggested by Eriksson ${ }^{36}$. Possibly, this is attributable to differences in experimental setup, as 
the current study was performed on mice without a diet and at a younger age than the mice used by Eriksson, resulting in a less developed vasa vasorum. On the other hand, it should be noted that Ephrin-B2 is known to be expressed on angiogenic endothelium, and therefore could account for the increased EphrinB2 staining. However, since there was no response to venous ligation, it is conceivable that the vasa vasorum in fact may drain on the carotid artery, acting comparable to an arterial shunt. This would explain the low flow, which could be a consequence of a small pressure gradient over the vasa vasorum, although the latter may also be influenced by pulsation caused by systolic and diastolic phases of the cardiac cycle.

Whereas plaque-associated microvessels were primarily located in the adventitia surrounding the atherosclerotic lesion, vessel-like structures were sporadically detected within the plaque itself (Supplemental Table II). Surprisingly, these structures did not originate from the vasa vasorum, since microvessels did not traverse the media. These findings oppose observations in human plaques ${ }^{8}$. Of note, incidental sprouting from the luminal endothelium was observed, yet these intraplaque vascular structures did not seem to be functionally perfused in vivo. Lucerna et al showed that overexpression of vascular endothelial growth factor (VEGF) in mice led to more luminal invaginations, accompanied by an increased leukocyte influx into the plaque ${ }^{13}$. Therefore, intraplaque microvascular structures, even when originating from the lumen, may contribute to the progression of atherosclerotic plaques in mice. Additionally, intimal microvessels are suggested to be causal for IPH, although no causal link between IPH and plaque vascularization could be discerned. The observed occurrence of IPH was within the range described in e.g. the studies from Rosenfeld ${ }^{49}$, Calara ${ }^{50}$, and Johnson ${ }^{51}$, which however show a large variation in the occurrence of IPH (3.6-75\%). Generally, proof of a causal relationship between vascularization and intraplaque hemorrhage is lacking in murine models, which is substantiated by our current study. The data however are suggestive for a contribution of vasa vasorum to IPH, or plaque instability in general, although vasa vasorum presence does not infer occurrence of IPH.

Even though this murine model does not completely mimic human atherosclerosis, as it only shows a limited, yet consistent occurrence of (intraplaque) vascularization, it provides new insights into the potential role of vasa vasorum in human atherosclerosis. Importantly, a number of features 
found in our study are consistent with previous studies in human atherosclerosis. For example, human plaque microvessels have been associated with ingress of leukocytes and erythrocytes and with leakage of albumin ${ }^{2}$. In this study, we established increased leukocyte adhesion and extravasation from adventitial microvessels, as well as an increased permeability of microvessels for $70 \mathrm{kDa}$ dextrans, resembling albumin leakage in human atherosclerosis. Interestingly, and in contrast to our model, human plaque microvessels generally seem to have a more instable microvascular phenotype and show disrupted inter-endothelial junctions ${ }^{10}$, especially in intraplaque microvessels. Hence, it would be interesting to investigate functional features of plaque neovascularization in other murine models, which have already described intraplaque microvessels or more extensive vasa vasorum expansion ${ }^{15,52}$.

In summary, using a novel in vivo imaging strategy we were able to study plaque-associated microvessels in mice and quantify its functional characteristics. The plaque-associated vasa vasorum demonstrated a significantly reduced blood flow, blood flow velocity, as well as an increase in leukocyte adhesion, leukocyte transmigration and endothelial permeability as compared to control microvasculature. In conjunction with the ongoing angiogenic activity, these features would support progression of atherosclerotic lesions. Further studies will help to unravel by which means microvascular function in the vasa vasorum may be normalized. 


\section{Supplemental Tables \& Figures}

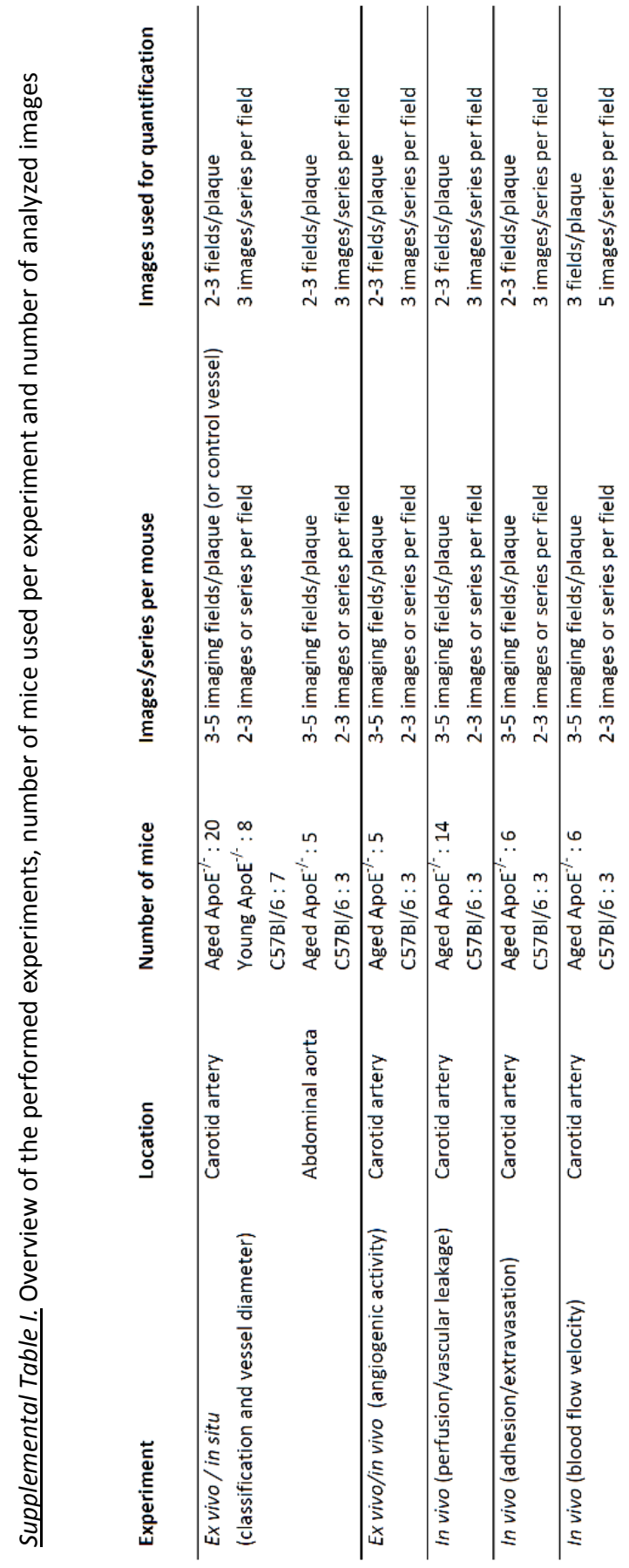


Supplemental Table II. Prevalence of intraplaque microvessels observed ex vivo in the carotid artery in the various experimental groups

\begin{tabular}{ccccc} 
& & \multicolumn{3}{c}{ Intraplaque vascularization } \\
\cline { 3 - 5 } Genotype & Number of mice & No & Yes & \% present \\
\hline $\mathrm{C} 57 \mathrm{BI} / 6(>40 \mathrm{wks})$ & 7 & 7 & 0 & 0 \\
$\mathrm{ApoE}^{-/}(<16 \mathrm{wks})$ & 8 & 8 & 0 & 0 \\
$\mathrm{ApoE}^{-/-}(>40 \mathrm{wks})$ & 20 & 14 & 6 & 30
\end{tabular}

$P=0.1412 ;$ Fisher's exact test; young vs. aged $\mathrm{ApoE}^{-1}$

Supplemental Table III. Quantification of the average blood flow velocity and accompanying average microvessel diameter of vasa vasorum and control microvessels of similar diameter

\begin{tabular}{|c|c|c|c|}
\hline & \multicolumn{3}{|c|}{ Diameter ( $\mu \mathrm{m})$} \\
\hline & Plaque microvessels & Control microvessels & $P$ value \\
\hline Total & $10.43( \pm 0.44)$ & $8.94( \pm 0.081)$ & N.S. \\
\hline Large microvessels (>10 $\mu \mathrm{m})$ & $18.52( \pm 0.33)$ & $9.16( \pm 0.02)$ & N.S. \\
\hline Small microvessels $(<10 \mu \mathrm{m})$ & $5.87( \pm 0.02)$ & $5.91( \pm 0.14)$ & N.S. \\
\hline
\end{tabular}

\begin{tabular}{rccc} 
& \multicolumn{3}{c}{ Blood flow velocitv $\left(\mathrm{mm} . \mathrm{s}^{-1}\right)$} \\
\cline { 2 - 4 } & Plaque microvessels & Control microvessels & P value \\
\hline Total & $0.14( \pm 0.012)$ & $0.30( \pm 0.013)$ & $<0.0001$ \\
Large microvessels $(>10 \mu \mathrm{m})$ & $0.28( \pm 0.026)$ & $0.33( \pm 0.013)$ & 0.0002 \\
Small microvessels $(<10 \mu \mathrm{m})$ & $0.06( \pm 0.002)$ & $0.15( \pm 0.017)$ & $<0.0001$ \\
\hline
\end{tabular}



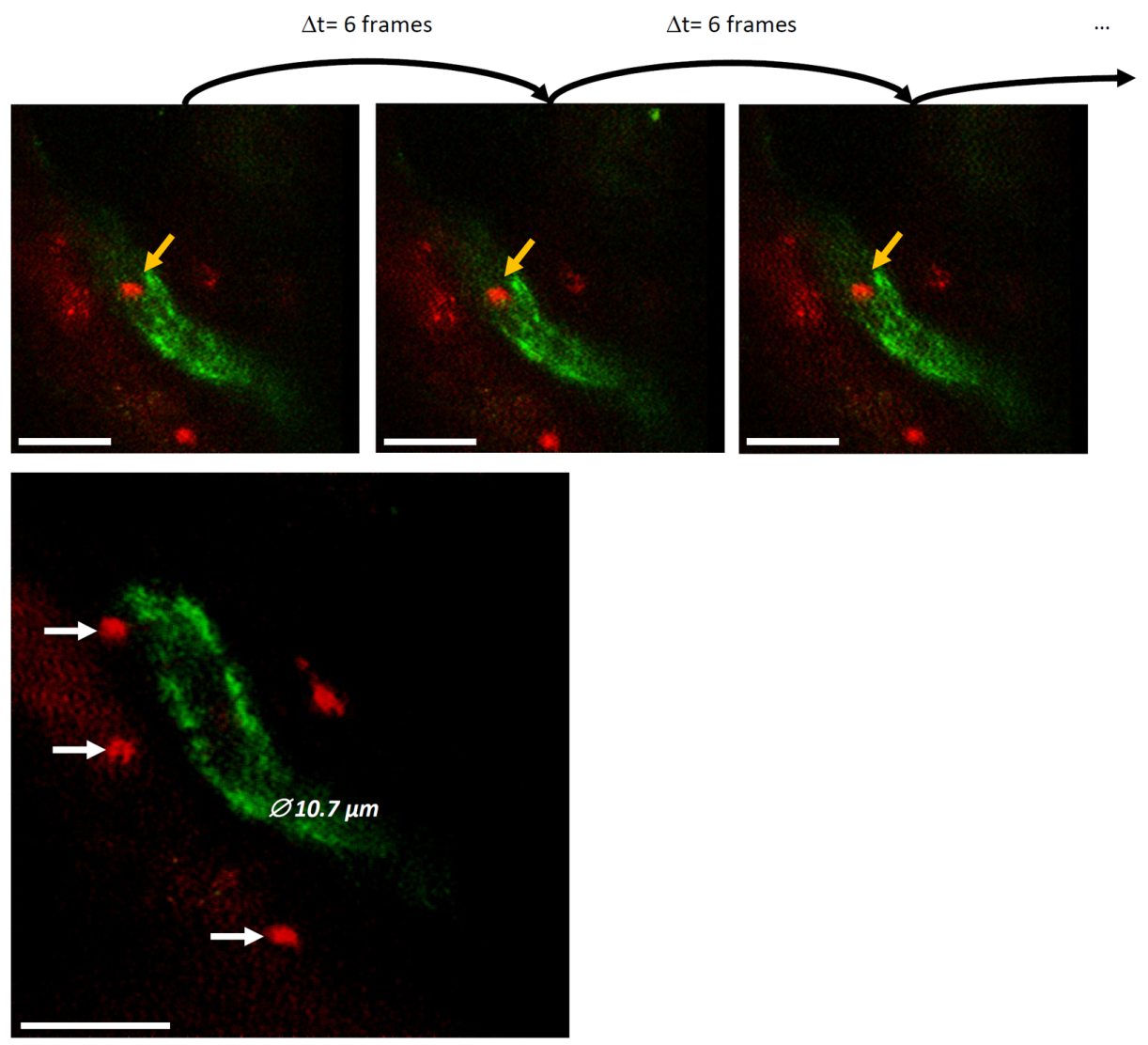

Supplemental Figure I. Occasional adhesion of leukocytes (red), defined as cells showing the same localization $>30$ seconds at identical focal planes, to the vessel wall (green) was observed (yellow arrows; $A-C, \Delta t=6$ frames). In a different focal plane, extravasated leukocytes could be detected (white arrows; D). Bars indicate $25 \mu \mathrm{m}$. 

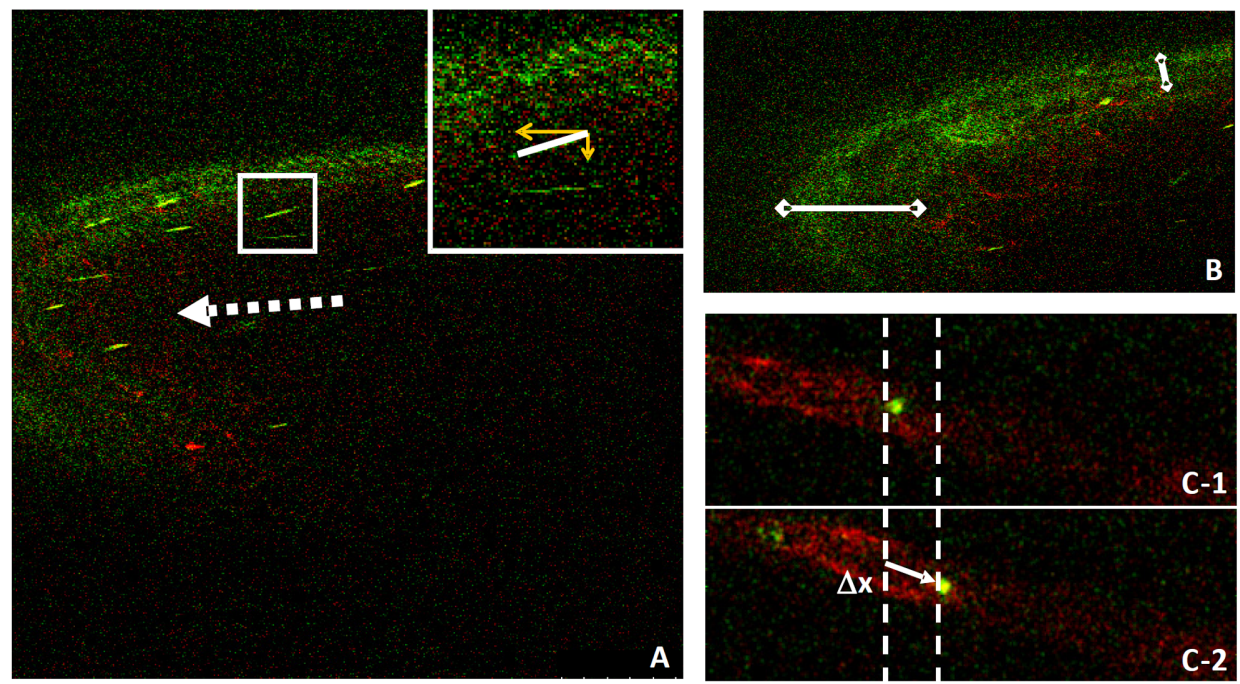

Supplemental Figure II. High speed imaging at $8000 \mathrm{~Hz}$ allows recording and calculation of blood flow velocity in both larger vessels (A and B) and microvessels (C). In the carotid artery, blood flow velocity can be derived from the distance that e.g. leukocytes or platelets travel during image acquisition (A), taking into account differences in delay time in the $x$ - and $y$-direction during imaging (inset) and the angle of imaging compared to the angle of the vessel (B). Dotted arrow indicates flow direction. For microvessels, which exhibit lower blood flow velocity, the distance travelled can be determined between two frames ( $C-1$ and $C-2, \Delta t=60 \mathrm{~ms}$ ). 

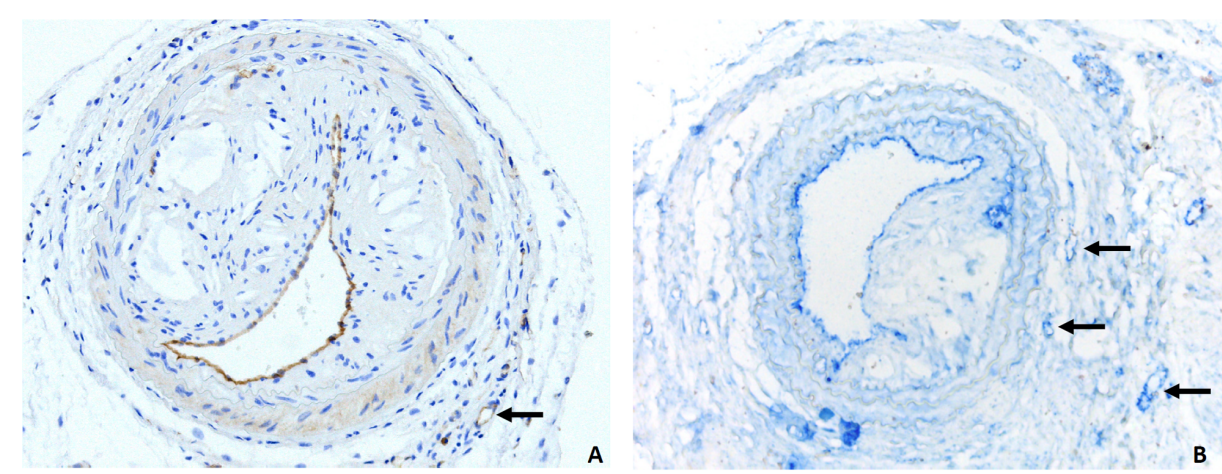

Supplemental Figure III. Presence of adventitial microvessels surrounding the plaque was confirmed by immunohistochemistry. Both $\operatorname{CD} 31$ (A) and vWf (B) were able to detect vasa vasorum vessels (arrows).

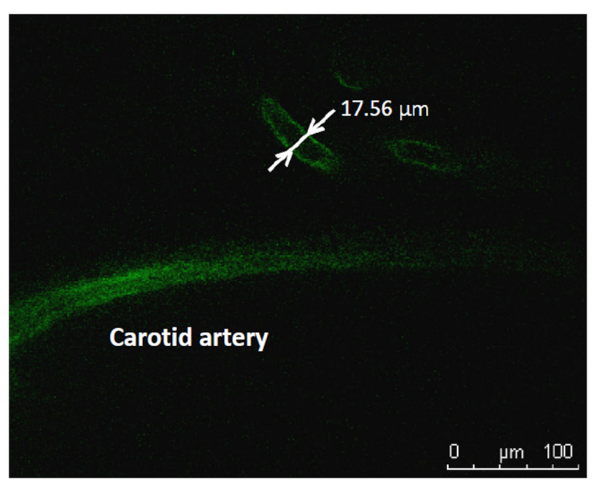

BEFORE

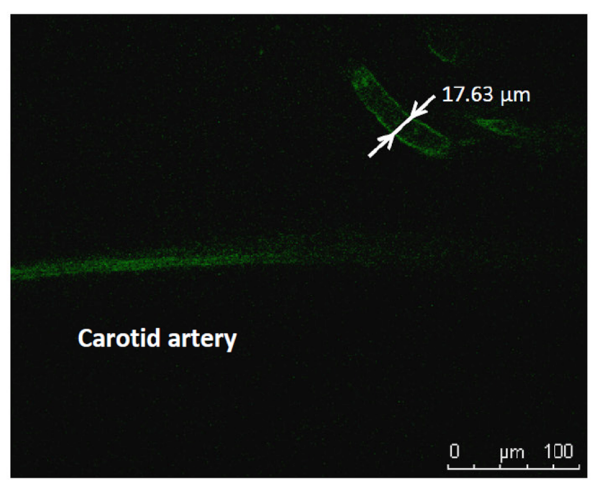

AFTER

Supplemental Figure IV. No changes in microvascular diameter were observed after bilateral ligation of the jugular vein (17.56 $\pm 0.55 \mu \mathrm{m}$ before, $17.63 \pm 0.38 \mu \mathrm{m}$ after ligation) arguing a lack of venous connections of the plaque-associated vasa vasorum. 

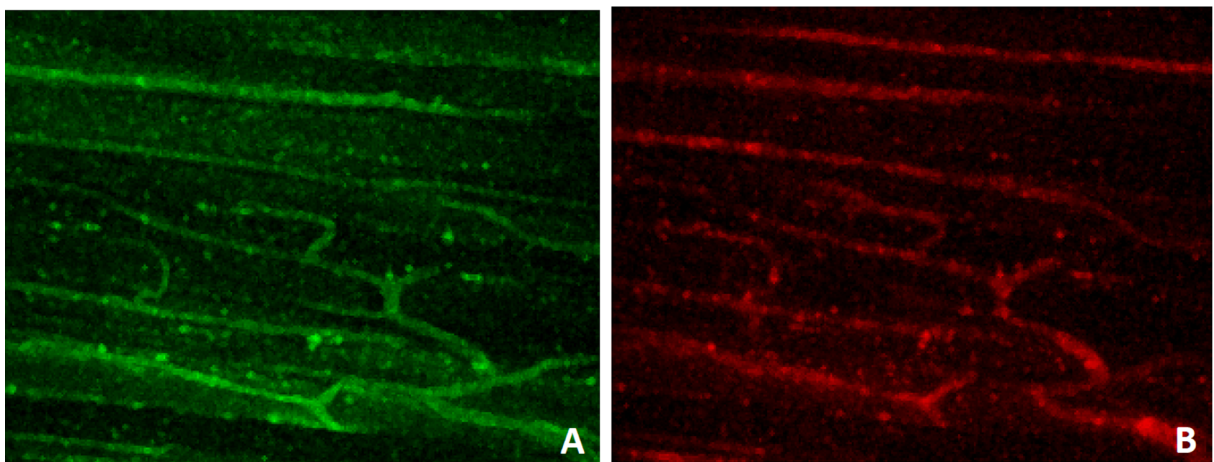

Supplemental Figure $V$. WGA-FITC staining in sternohyoid muscle showing constitutive expression of WGA (A, green) in the microvessels of the muscle (B, red).
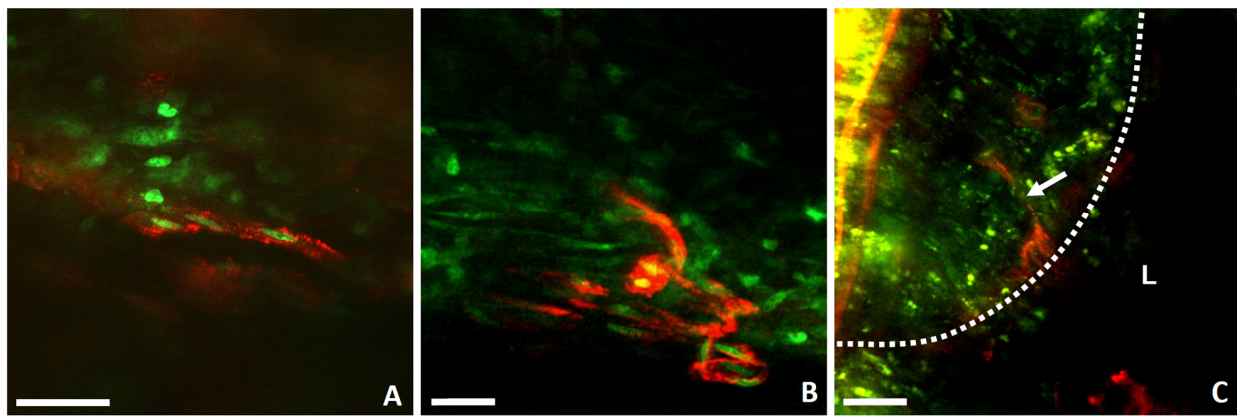

Supplemental Figure VI. Microvascular structures (red) were detected inside plaques (green, nuclei (A and $B$ ); lipids (C)) of aged $A_{p o E^{-/-}}$mice. Intraplaque vessels ranged from short sprouts (A) to more extensive vascular structures within the plaque (B). The intraplaque vessels were present in 6 out of 20 examined plaques, and seemed to originate predominantly from the luminal endothelium (C, arrow). Dotted line outlines the outer perimeter of the plaque. Bars indicate $25 \mu \mathrm{m}$, " $\mathrm{L}$ " indicates the lumen of the carotid artery. 


\section{Supplemental Video Files}

\section{Supplemental Video 1}

Link at:

http://atvb.ahajournals.org/content/suppl/2012/12/13/ATVBAHA.112.300087.DC1/ATV201659.Video1.avi

3D rendering showing the carotid artery, directly proximal from the bifurcation. Blue depicts collagen as determined by SHG, green depicts CD31 (adventitia), as well as plaque autofluorescence (within the artery). Box size: $738 \times 738 \times 235 \mu^{3}$.

\section{Supplemental Video 2}

Link at:

http://atvb.ahajournals.org/content/suppl/2012/12/13/ATVBAHA.112.300087.DC1/ATV201659.Video2.avi

In vivo MPLSM imaging of the vasa vasorum network (green) surrounding the carotid artery at the atherosclerotic plaque. Vasa vasorum network was located approximately 15-20 $\mu \mathrm{m}$ from the outer elastic lamina. Box size: $434 \times 434 \times 110 \mu \mathrm{m}^{3}$.

\section{Supplemental Video 3}

Link at:

http://atvb.ahajournals.org/content/suppl/2012/12/13/ATVBAHA.112.300087.DC1/ATV201659.Video3.avi

3D reconstruction ( $A$ and $B$ ) of in situ z-scan showing leukocytes (red) adhering to and extravasated from the vasa vasorum (green), as well as cells in the process of extravasation. MPLSM allowed differentiation of morphologically distinct cell types, e.g. pseudopodia-rich cells resembling a dendritic morphology. Box size: $140 \times 140 \times 130 \mu \mathrm{m}^{3}$. 


\section{References}

1. Sluimer JC, Daemen MJ. Novel concepts in atherogenesis: Angiogenesis and hypoxia in atherosclerosis. J Pathol. 2009;218:7-29

2. Virmani R, Kolodgie FD, Burke AP, Finn AV, Gold HK, Tulenko TN, Wrenn SP, Narula J. Atherosclerotic plaque progression and vulnerability to rupture: Angiogenesis as a source of intraplaque hemorrhage. Arterioscler Thromb Vasc Biol. 2005;25:2054-2061

3. Köster W. Endarteritis and arteritis. Berl Klin Wochenschr. 1876;13:343-345

4. Gössl M, Versari D, Mannheim D, Ritman EL, Lerman LO, Lerman A. Increased spatial vasa vasorum density in the proximal lad in hypercholesterolemia-implications for vulnerable plaque-development. Atherosclerosis. 2007; 192:246-252

5. Moreno PR, Purushothaman KR, Fuster V, Echeverri D, Truszczynska H, Sharma SK, Badimon JJ, O'Connor WN. Plaque neovascularization is increased in ruptured atherosclerotic lesions of human aorta: Implications for plaque vulnerability. Circulation. 2004;110:2032-2038

6. de Boer OJ, van der Wal AC, Teeling $P$, Becker AE. Leucocyte recruitment in rupture prone regions of lipid-rich plaques: $A$ prominent role for neovascularization? Cardiovasc Res. 1999;41:443-449

7. Jeziorska M, Woolley DE. Local neovascularization and cellular composition within vulnerable regions of atherosclerotic plaques of human carotid arteries. J Pathol. 1999;188:189-196

8. Kumamoto $M$, Nakashima $Y$, Sueishi K. Intimal neovascularization in human coronary atherosclerosis: Its origin and pathophysiological significance. Hum Pathol. 1995;26:450-456

9. Sluimer JC, Gasc JM, van Wanroij JL, Kisters N, Groeneweg M, Sollewijn Gelpke MD, Cleutjens JP, van den Akker LH, Corvol P, Wouters BG, Daemen MJ, Bijnens AP. Hypoxia, hypoxia-inducible transcription factor, and macrophages in human atherosclerotic plaques are correlated with intraplaque angiogenesis. J Am Coll Cardiol. 2008;51:1258-1265

10. Sluimer JC, Kolodgie FD, Bijnens AP, Maxfield K, Pacheco E, Kutys B, Duimel H, Frederik PM, van Hinsbergh VW, Virmani R, Daemen MJ. Thin-walled microvessels in human coronary atherosclerotic plaques show incomplete endothelial junctions relevance of compromised structural integrity for intraplaque microvascular leakage. J Am Coll Cardiol. 2009;53:1517-1527

11. Moos MP, John N, Grabner R, Nossmann S, Gunther B, Vollandt R, Funk CD, Kaiser B, Habenicht AJ. The lamina adventitia is the major site of immune cell accumulation in standard chow-fed apolipoprotein e-deficient mice. Arterioscler Thromb Vasc Biol. 2005;25:2386-2391

12. Celletti FL, Waugh JM, Amabile PG, Brendolan A, Hilfiker PR, Dake MD. Vascular endothelial growth factor enhances atherosclerotic plaque progression. Nat Med. 2001;7:425-429

13. Lucerna $M$, Zernecke $A$, de Nooijer R, de Jager SC, Bot I, van der Lans $C$, Kholova I, Liehn EA, van Berkel TJ, Yla-Herttuala S, Weber C, Biessen EA. 
Vascular endothelial growth factor-a induces plaque expansion in apoe knockout mice by promoting de novo leukocyte recruitment. Blood. 2007;109:122129

14. Luttun A, Tjwa M, Carmeliet P. Placental growth factor (plgf) and its receptor flt-1 (vegfr-1): Novel therapeutic targets for angiogenic disorders. Ann $N Y$ Acad Sci. 2002;979:80-93

15. Moulton KS, Olsen BR, Sonn S, Fukai N, Zurakowski D, Zeng X. Loss of collagen xviii enhances neovascularization and vascular permeability in atherosclerosis. Circulation. 2004;110:1330-1336

16. Tanaka K, Nagata D, Hirata Y, Tabata Y, Nagai R, Sata M. Augmented angiogenesis in adventitia promotes growth of atherosclerotic plaque in apolipoprotein e-deficient mice. Atherosclerosis. 2011;215:366-373

17. Hauer $A D$, van Puijvelde $G H$, Peterse $N$, de Vos $P$, van Weel V, van Wanrooij EJ, Biessen EA, Quax PH, Niethammer AG, Reisfeld RA, van Berkel TJ, Kuiper J. Vaccination against vegfr2 attenuates initiation and progression of atherosclerosis. Arterioscler Thromb Vasc Biol. 2007;27:2050-2057

18. Moulton KS, Heller E, Konerding MA, Flynn E, Palinski W, Folkman J. Angiogenesis inhibitors endostatin or tnp-470 reduce intimal neovascularization and plaque growth in apolipoprotein e-deficient mice. Circulation. 1999;99:1726-1732

19. Moulton KS, Vakili K, Zurakowski D, Soliman M, Butterfield C, Sylvin E, Lo KM, Gillies S, Javaherian K, Folkman J. Inhibition of plaque neovascularization reduces macrophage accumulation and progression of advanced atherosclerosis. Proc Natl Acad Sci U S A. 2003;100:4736-4741

20. Petrovan RJ, Kaplan CD, Reisfeld RA, Curtiss LK. DNA vaccination against vegf receptor 2 reduces atherosclerosis in Idl receptor-deficient mice. Arterioscler Thromb Vasc Biol. 2007;27:1095-1100

21. Calcagno C, Cornily JC, Hyafil F, Rudd JH, Briley-Saebo KC, Mani V, Goldschlager G, Machac J, Fuster V, Fayad ZA. Detection of neovessels in atherosclerotic plaques of rabbits using dynamic contrast enhanced mri and 18f-fdg pet. Arterioscler Thromb Vasc Biol. 2008;28:1311-1317

22. Winter PM, Morawski AM, Caruthers SD, Fuhrhop RW, Zhang H, Williams TA, Allen JS, Lacy EK, Robertson JD, Lanza GM, Wickline SA. Molecular imaging of angiogenesis in early-stage atherosclerosis with alpha(v)beta3-integrintargeted nanoparticles. Circulation. 2003;108:2270-2274

23. Langheinrich AC, Michniewicz A, Sedding DG, Walker G, Beighley PE, Rau WS, Bohle RM, Ritman EL. Correlation of vasa vasorum neovascularization and plaque progression in aortas of apolipoprotein e(-/-)/low-density lipoprotein(/-) double knockout mice. Arterioscler Thromb Vasc Biol. 2006;26:347-352

24. Megens RT, Reitsma S, Schiffers PH, Hilgers RH, De Mey JG, Slaaf DW, oude Egbrink MG, van Zandvoort MA. Two-photon microscopy of vital murine elastic and muscular arteries. Combined structural and functional imaging with subcellular resolution. J Vasc Res. 2007;44:87-98

25. van Zandvoort M, Engels W, Douma K, Beckers L, Oude Egbrink M, Daemen M, Slaaf DW. Two-photon microscopy for imaging of the (atherosclerotic) vascular wall: A proof of concept study. J Vasc Res. 2004;41:54-63 
26. Buehler A, van Zandvoort MA, Stelt BJ, Hackeng TM, Schrans-Stassen BH, Bennaghmouch A, Hofstra L, Cleutjens JP, Duijvestijn A, Smeets MB, de Kleijn $D P$, Post MJ, de Muinck ED. Cngr: A novel homing sequence for cd13/apn targeted molecular imaging of murine cardiac angiogenesis in vivo. Arterioscler Thromb Vasc Biol. 2006;26:2681-2687

27. Oostendorp M, Douma K, Wagenaar A, Slenter JM, Hackeng TM, van Zandvoort MA, Post MJ, Backes WH. Molecular magnetic resonance imaging of myocardial angiogenesis after acute myocardial infarction. Circulation. 2010;121:775-783

28. Megens RT, Reitsma S, Prinzen L, oude Egbrink MG, Engels W, Leenders PJ, Brunenberg EJ, Reesink KD, Janssen BJ, ter Haar Romeny BM, Slaaf DW, van Zandvoort MA. In vivo high-resolution structural imaging of large arteries in small rodents using two-photon laser scanning microscopy. J Biomed Opt. 2010;15:011108

29. Yu W, Braz JC, Dutton AM, Prusakov P, Rekhter M. In vivo imaging of atherosclerotic plaques in apolipoprotein e deficient mice using nonlinear microscopy. J Biomed Opt. 2007;12:054008

30. Kamoun WS, Chae SS, Lacorre DA, Tyrrell JA, Mitre M, Gillissen MA, Fukumura D, Jain RK, Munn LL. Simultaneous measurement of rbc velocity, flux, hematocrit and shear rate in vascular networks. Nat Methods. 2010;7:655660

31. Duff SE, Li C, Garland JM, Kumar S. Cd105 is important for angiogenesis: Evidence and potential applications. FASEB J. 2003;17:984-992

32. Luque A, Slevin M, Turu MM, Juan-Babot O, Badimon L, Krupinski J. Cd105 positive neovessels are prevalent in early stage carotid lesions, and correlate with the grade in more advanced carotid and coronary plaques. J Angiogenes Res. 2009;1:6

33. Kuijpers MJ, Pozgajova M, Cosemans JM, Munnix IC, Eckes B, Nieswandt B, Heemskerk JW. Role of murine integrin alpha2beta1 in thrombus stabilization and embolization: Contribution of thromboxane a2. Thromb Haemost. 2007;98:1072-1080

34. Dirkx AE, Oude Egbrink MG, Kuijpers MJ, van der Niet ST, Heijnen VV, Boumater Steege JC, Wagstaff J, Griffioen AW. Tumor angiogenesis modulates leukocyte-vessel wall interactions in vivo by reducing endothelial adhesion molecule expression. Cancer Res. 2003;63:2322-2329

35. Schmid B, Schindelin J, Cardona A, Longair M, Heisenberg M. A high-level 3d visualization api for java and imagej. BMC Bioinformatics. 2010;11:274

36. Eriksson EE. Intravital microscopy on atherosclerosis in apolipoprotein edeficient mice establishes microvessels as major entry pathways for leukocytes to advanced lesions. Circulation. 2011;124:2129-2138

37. van den Berg BM, Vink H, Spaan JA. The endothelial glycocalyx protects against myocardial edema. Circ Res. 2003;92:592-594

38. Alon R, Ley K. Cells on the run: Shear-regulated integrin activation in leukocyte rolling and arrest on endothelial cells. Curr Opin Cell Biol. 2008;20:525-532 
39. Dekker RJ, van Thienen JV, Rohlena J, de Jager SC, Elderkamp YW, Seppen J, de Vries CJ, Biessen EA, van Berkel TJ, Pannekoek $H$, Horrevoets AJ. Endothelial klf2 links local arterial shear stress levels to the expression of vascular tone-regulating genes. Am J Pathol. 2005;167:609-618

40. Mestas J, Ley K. Monocyte-endothelial cell interactions in the development of atherosclerosis. Trends Cardiovasc Med. 2008;18:228-232

41. Jeziorska M, Woolley DE. Neovascularization in early atherosclerotic lesions of human carotid arteries: Its potential contribution to plaque development. Hum Pathol. 1999;30:919-925

42. Doyle B, Caplice N. Plaque neovascularization and antiangiogenic therapy for atherosclerosis. J Am Coll Cardiol. 2007;49:2073-2080

43. Lobbes MB, Miserus RJ, Heeneman S, Passos VL, Mutsaers PH, Debernardi N, Misselwitz B, Post M, Daemen MJ, van Engelshoven JM, Leiner T, Kooi ME. Atherosclerosis: Contrast-enhanced $\mathrm{mr}$ imaging of vessel wall in rabbit model-comparison of gadofosveset and gadopentetate dimeglumine. Radiology. 2009;250:682-691

44. Zhang Y, Cliff WJ, Schoefl GI, Higgins G. Immunohistochemical study of intimal microvessels in coronary atherosclerosis. Am J Pathol. 1993;143:164-172

45. Grabner R, Lotzer K, Dopping S, Hildner M, Radke D, Beer M, Spanbroek R, Lippert B, Reardon CA, Getz GS, Fu YX, Hehlgans T, Mebius RE, van der Wall M, Kruspe D, Englert C, Lovas A, Hu D, Randolph GJ, Weih F, Habenicht AJ. Lymphotoxin beta receptor signaling promotes tertiary lymphoid organogenesis in the aorta adventitia of aged apoe-/- mice. The Journal of experimental medicine. 2009;206:233-248

46. Bot I, Jukema JW, Lankhuizen IM, van Berkel TJ, Biessen EA. Atorvastatin inhibits plaque development and adventitial neovascularization in apoe deficient mice independent of plasma cholesterol levels. Atherosclerosis. 2011;214:295-300

47. Li ZJ, Wang ZZ, Zheng YZ, Xu B, Yang RC, Scadden DT, Han ZC. Kinetic expression of platelet endothelial cell adhesion molecule-1 (pecam-1/cd31) during embryonic stem cell differentiation. J Cell Biochem. 2005;95:559-570

48. RayChaudhury A, Elkins M, Kozien D, Nakada MT. Regulation of pecam-1 in endothelial cells during cell growth and migration. Exp Biol Med (Maywood). 2001;226:686-691

49. Rosenfeld ME, Polinsky P, Virmani R, Kauser K, Rubanyi G, Schwartz SM. Advanced atherosclerotic lesions in the innominate artery of the apoe knockout mouse. Arteriosclerosis, thrombosis, and vascular biology. 2000;20:2587-2592

50. Calara F, Silvestre M, Casanada F, Yuan N, Napoli C, Palinski W. Spontaneous plaque rupture and secondary thrombosis in apolipoprotein e-deficient and Idl receptor-deficient mice. The Journal of pathology. 2001;195:257-263

51. Johnson JL, Jackson CL. Atherosclerotic plaque rupture in the apolipoprotein e knockout mouse. Atherosclerosis. 2001;154:399-406

52. Khatri JJ, Johnson C, Magid R, Lessner SM, Laude KM, Dikalov SI, Harrison DG, Sung HJ, Rong Y, Galis ZS. Vascular oxidant stress enhances progression and angiogenesis of experimental atheroma. Circulation. 2004;109:520-525 
PRO-ATHEROGENIC FEATURES OF PLAQUE-ASSOCIATED VASA VASORUM IN VIVO 


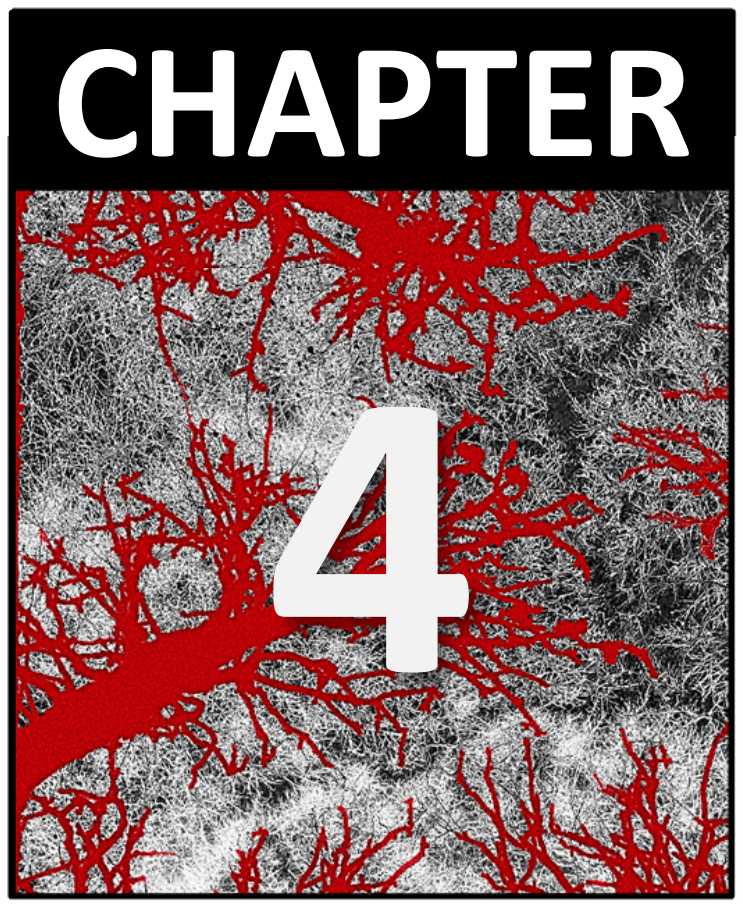




\title{
Flow-dependent redistribution of endothelial junctional adhesion molecule-A in atherosclerosis causes increased leukocyte recruitment
}

\author{
Martin M.N. Schmitt ${ }^{*}$, Rory R. Koenen ${ }^{*}$, Remco T. A. Megens, \\ Timo Rademakers, Alma Zernecke, Marc A.M.J. van Zandvoort, \\ Tilman M. Hackeng, and Christian Weber \\ * authors contributed equally
}

Part of the manuscript "A cell-specific role of junctional adhesion molecule-A in flow-dependent atherosclerosis", submitted 


\section{Abstract}

Junctional adhesion molecule (JAM)-A is an immunoglobulin superfamily protein mainly located at endothelial cell junctions. Engaging in homophilic and heterophilic interactions, JAM-A plays an important role in regulating endothelial permeability, and inflammatory and atherogenic leukocyte extravasation. Little is known about the contributions of endothelial JAM-A to atherogenesis and the underlying mechanisms.

Here, we show that impaired JAM-A expression due to genetic deletion in endothelial cells reduced atherosclerotic lesion formation, lesional $\mathrm{T}$ cell content, and macrophage content in Apolipoprotein-E deficient (ApoE ${ }^{-/}$) mice. Multiphoton laser scanning microscopy of atherosclerotic carotid arteries ex vivo revealed a focal concentration and redistribution of endothelial JAM-A to the luminal surface in lesion-prone areas or under conditions of disturbed flow. Deficiency in endothelial JAM-A furthermore reduced leukocyte recruitment in vivo by approximately $50 \%$. Endothelial barrier function as measured by permeability to $70 \mathrm{kDa}$ and $150 \mathrm{kDa}$ dextran was unaffected by endothelial JAM-A deficiency.

Our data identified specific and targetable functions of endothelial JAM-A in atherosclerosis, which were mediated by its upregulation and redistribution under atherogenic conditions, which in turn facilitated enhanced leukocyte recruitment. 


\section{Introduction}

Junctional adhesion molecule (JAM)-A, a member of the immunoglobulin (IgG) superfamily, displays a wide variety of functions in cell polarity ${ }^{1}$ and barrier function via homophilic interactions ${ }^{2}$, stem cell adhesion and differentiation ${ }^{3}$, as well as leukocyte trafficking and recruitment ${ }^{4}$. JAM-A is located mainly in the junctions of endothelial cells (ECS) and epithelial cells, but is also expressed by leukocytes and $\mathrm{CD}_{3} 4^{+}$cells ${ }^{5}$. In particular, JAM-A mediates the adhesion and transmigration via heterophilic interactions with the $\alpha \mathrm{L} \beta 2$ integrin LFA- ${ }^{6-8}$. Furthermore, dendritic cell trafficking to the lymph nodes is increased in mice with somatic but not endothelial JAM-A deficiency, indicating a specific role in dendritic cell migration ${ }^{9}$. Additional findings demonstrated a role of leukocyte JAM-A in promoting de-adhesion ${ }^{10}$. In the absence of JAM-A, the directional motility of neutrophils was reduced, leading to incomplete transmigration with neutrophils stuck between the endothelial cell layer and the basement membrane ${ }^{11}$. Whereas endothelial JAM-A has been implicated in the sequential steps mediating stimulus-specific neutrophil transmigration ${ }^{12}$, recent data confirm that JAM-A on neutrophils can promote their chemotaxis by controlling integrin internalization and recycling ${ }^{10}$. Amongst the intracellular interactions of endothelial JAM-A, binding to the PDZ domain protein ZO-1 is important in facilitating anchorage to the cytoskeleton ${ }^{13}$. Upon co-stimulation with cytokines, endothelial JAM-A is redistributed to the luminal surface ${ }^{14,15}$ and specifically to the site of leukocyte transmigration as part of lateral border recycling compartments ${ }^{16}$.

Convincing evidence has recently emerged from various models to indicate that JAM-A-mediated leukocyte transmigration plays an important role in inflammatory disease. For example, JAM-A-deficient mice displayed reduced leukocyte infiltration in models of peritonitis, as well as in hepatic and myocardial ischemia-reperfusion injury in liver ${ }^{11,17}$. In models of vascular disease, somatic JAM-A deficiency is shown to protect against neointimal hyperplasia after wire-injury of the carotid artery in $\mathrm{ApoE}^{-1-}$ mice ${ }^{18}$. Accordingly, increased levels of JAM-A transcripts and protein have been demonstrated in human plaque biopsies and in atherosclerotic aortas of mice ${ }^{19}$. The expression of JAM-A on inflamed and early atherosclerotic endothelium has been implicated in the recruitment of mononuclear cells 


\section{CHAPTER 4}

through an inhibition with soluble JAM-A, suggesting a functional involvement in atherogenesis ${ }^{20}$.

However, little is known about the direct influence and effect of JAM-A deficiency in atherosclerosis. Given the differential effects of JAM-A deficiency on various cell types relevant in atherosclerosis, the cell-specific contributions of JAM-A to atherosclerotic lesion formation and inflammatory phenotype is of particular interest. In this study, the role of endothelial JAM-A in atherosclerosis is delineated, using $\mathrm{ApoE}^{-/-}$mice with an inducible, endothelial cell-specific deficiency in JAM-A. We demonstrate that endothelial JAM-A is crucial in promoting atherosclerotic lesion formation. Endothelial JAM-A is redistributed as a result of disturbed flow, and facilitates leukocyte migration into the plaque. 


\section{Methods}

\section{Atherosclerotic mouse models with endothelial JAM-A depletion}

A genetic reduction of endothelial JAM-A was achieved by crossing JAM- $A^{\mathrm{fl} / \mathrm{fl}}$ ApoE ${ }^{-1-}$ mice with VeCad $\mathrm{CreRT}^{2}$ mice (both C57BI/6) to obtain VeCad CreRT ${ }^{2}$ $\mathrm{JAM}^{\mathrm{Af}} \mathrm{A}^{\mathrm{fl} / \mathrm{fl}} \mathrm{ApoE}^{-/-}$mice (17072878). At an age of 3 weeks, 5 daily i.p. injections of the estradiol analog tamoxifen $(0.04 \mathrm{mg} / \mathrm{g}$ bodyweight) dissolved in miglyol (number 812) were administered to $\mathrm{Cre}^{+}$deletion (eJAM- $\mathrm{A}^{-/-} \mathrm{ApoE}^{-{ }^{-}}$) and to Cre control (eJAM-A ${ }^{+/+} \mathrm{ApoE}^{-/}$) mice. Genetic depletion of JAM-A was checked via flow cytometry analysis after 2 weeks, resulting in a $\pm 30 \%$ reduction in endothelial JAM-A, but with no difference in leukocytic JAM-A levels in $\mathrm{Cre}^{+}$ mice compared to control Cre- mice. At comparable ages, female littermates were subjected for 12 weeks to atherogenic diet ( $21 \%$ fat, $0.15 \%$ cholesterol, Altromin) with subsequent sacrifice for quantification of atherosclerotic lesion formation. Animal experiments were approved by local authorities and complied with the German animal protection law (Landesamt für Natur, Umwelt und Verbraucherschutz Nordrhein-Westfalen, Recklinghausen, Germany).

\section{Quantification and immunohistochemical analysis of atherosclerosis}

The extent of atherosclerosis was assessed in aortic roots and on thoracoabdominal aortas by staining according to Elastica Van Gieson protocol (EVG) and for lipid depositions with oil red O (ORO), respectively, ${ }^{21}$ and was quantified by computerized image analysis (Diskus Software, Hilgers) and Leica Qwin Imaging software. Briefly, atherosclerotic lesions of aortic roots were measured in $5 \mu \mathrm{m}$ transverse paraffin sections through the heart and the aortic roots. The thoracoabdominal aorta was opened longitudinally and prepared en face, and the percentage of lipid deposition was calculated by dividing the stained area by the total thoracoabdominal aortic surface. The relative content of macrophages and $\mathrm{CD}^{+}{ }^{+} \mathrm{T}$ cells ${ }^{21}$ in the aortic root lesions was determined by mAb staining for MAC-2, CD3 (both AbD Serotec), and detection with FITC and Cy3-conjugated antibody (both Jackson ImmunoResearch Laboratories Inc.). Nuclei were counterstained by DAPI. Images were recorded with a Leica DMLB fluorescence microscope and CCD camera. 


\section{In vitro and in vivo monocyte transmigration assays}

$\mathrm{CD} 14^{+}$human monocytes and $\mathrm{CD} 115^{+}$mouse monocytes were freshly isolated from blood from healthy volunteers or from spleens of mice with desired genetic modification, respectively, for every experimental series using the Monocyte Isolation Kit II or the CD115 MicroBead Kit mouse (Miltenyi). Human monocytes at a concentration of $7.0 \times 10^{5}$ cells $/ \mathrm{ml}$ were applied on Human Aortic Endothelial Cells (HAoECs) under shear stress $\left(1.5 \mathrm{dyn} / \mathrm{cm}^{2}\right)$ using a syringe pump (WPI) in a laminar flow chamber for 5 minutes and flushed with assay medium for 30 minutes, while time lapse were recorded (Olympus, Cell ${ }^{\mathrm{M}}$ system and software). Mouse monocytes at a concentration of $2 \times 10^{6}$ cells $/ \mathrm{ml}$ were applied on stimulated mouse ECs under shear stress $\left(0.8 \mathrm{dyn} / \mathrm{cm}^{2}\right.$, BioFlux 200) for 10 minutes and flushed with assay medium for 30 minutes, while time lapse images were recorded. Monocytes showing complete transmigration were counted and related to adherent monocytes.

Right carotid arteries of eJAM- $\mathrm{A}^{-/-}$and eJAM- $\mathrm{A}^{+/+}$mice after treatment with tamoxifen were challenged 24 hours in vivo with $\mathrm{IL}-1 \beta(2 \mu \mathrm{g} / \mathrm{ml}$, BioLegend) carried by a periarterial slowly degrading pluronic gel to induce enhanced monocyte recruitment. Additionally, Alexa568-coupled anti-CD115 antibody ( $5 \mu \mathrm{g} /$ mouse, eBioscience) was administered intravenously. On the next day, anaesthetized mice were injected intravenously with anti-CD31 antibody ( $35 \mu \mathrm{g} /$ mouse total, BD Bioscience) and subsequently prepared for in vivo MPLSM by exposing the common carotid artery by dissection.

\section{Multiphoton microscopy of the carotid artery in vitro and in vivo}

MPLSM imaging was performed on a Leica SP5 imaging platform (Leica Microsystems) that integrates multiphoton microscopy with fast resonant scanning, and uses a Compact Ultrafast Ti:Sapphire Laser (Chameleon, Coherent, USA). An excitation wavelength of $810 \mathrm{~nm}$ was used in all experiments. Tissues were observed using a 20x NA 1.0 water immersion objective (HCX PL APO L, Leica Microsystems, Germany) with a numerical aperture of 1.00 and an integrated optical zoom mechanism allowing magnification up to 60x. Photo-multiplier tubes (PMT) were used to detect three spectral regions: PMT1: $400-470 \mathrm{~nm}$ for second harmonic generation (SHG); PMT2: 500-565 nm (FITC or Alexa488) and PMT3: 565-605 nm (PI, PE and Alexa568). In vivo MPLSM imaging was performed at a frame size of $400 \mathrm{x}$ 400 pixels (pixel size: $1.0882 \times 1.0882 \mu \mathrm{m}$ ), scanning at $8000 \mathrm{~Hz}$ for fast 
recording (20 frames/sec) and using a line average of 2 (10 frames/sec) for more detailed scans. Per time series, a total of 500 frames was recorded. After in vivo imaging, the animal was sacrificed and additional in situ imaging was performed to examine the vessel at higher resolution and an increased signalto-noise ratio without motion artifacts. In situ imaging was performed at a frame size of $512 \times 512$ pixels (pixel size: $0.847 \times 0.847 \mu \mathrm{m}$ ), scanning at 200 $\mathrm{Hz}$, and an interplanar distance of $1.00 \mu \mathrm{m}$.

Newly dissected plaque-prone and partially ligated ${ }^{22}$ carotid arteries were carefully mounted in a perfusion chamber and imaged using an Olympus FV1000MPE multiphoton system coupled to an Olympus BX61WI microscope. Therefore, mounted carotids were flushed and direct conjugated JAM-AAlexa488 (10 $\mu \mathrm{g} / \mathrm{ml}$, AbD serotec) and CD31-PE $(2 \mu \mathrm{g} / \mathrm{ml}, \mathrm{BD})$ antibodies were luminally applied under physiological pressure for $1 \mathrm{~h}$. After thorough washing, the vessel was pressurized again. Excitation laser (MaiTai HP) was tuned at 800 $\mathrm{nm}$ to visualize the fluorophores and SHG of collagen. An Olympus 20x (NA 0.95; water immersion) objective was used. An increase in magnification was achieved by internal optical zoom. For detection of emitted fluorescent signals, 3 PMTs were tuned as described above, ensuring minimal bleed-through. Separate images of $1,024 \times 1,024$ pixels were obtained from each PMT and combined into single RGB images. Images were collected in $x y$ directions, and series of $x y$ images were obtained at successive $1 \mu \mathrm{m}$ depth positions (z-stack) for reconstruction of 3D images. Image analysis was performed using ImagePro Analyzer 7.0 (Media Cybernetics) and MetaMorph (Molecular Devices).

\section{Measurement of vessel wall permeability}

Dissected carotid arteries from $\mathrm{C} 57 \mathrm{Bl} / 6, \mathrm{ApoE}^{-/-}$on a chow or Western type diet, or VeCad CreRT2 JAM-A $A^{\mathrm{fl} / \mathrm{fl}}$ ApoE ${ }^{-/-}\left(\mathrm{eJAM}-\mathrm{A}^{-/} \mathrm{ApoE}^{--}\right.$) on a Western type diet were mounted in a perfusion chamber and incubated with 70kDa dextran coupled to rhodamine-B and $150 \mathrm{kDa}$ dextran coupled to FITC, $0.35 \mathrm{mg} / \mathrm{ml}$ each, for one hour under exact $60 \mathrm{mmHg}$ pressure. Control carotids were also co-incubated with $50 \mu \mathrm{M}$ histamine. After subsequent defined washing of the vessels z-stacks were taken with an Olympus FV1000MPE multiphoton system coupled to an Olympus BX61WI microscope. All microscope setting were left unaltered throughout all measurements, besides $830 \mathrm{~nm}$ wavelength being used for rhodamine-B excitation and $800 \mathrm{~nm}$ wavelength being used for FITC excitation. Evaluation was performed using Image-Pro Analyzer 7.0 (Media 


\section{CHAPTER 4}

Cybernetics), therefore regions of interest comprising the area between internal elastic lamina and intermediate elastic lamina and between intermediate elastic lamina and external elastic lamina were determined and fluorescent intensities were calculated from desired channels ${ }^{23}$.

\section{Statistical Analysis}

Statistical analysis was performed using Prism 4.0 (GraphPad Software). Data were compared either by paired or unpaired Student's t-test or by 1-way ANOVA with Newman-Keuls post test. Differences with at least $p<0.05$ were considered as statistically significant. 


\section{Results}

Deficiency in endothelial JAM-A protected mice against atherosclerosis and reduced inflammatory cell content in lesions

The inhibition of mononuclear cell recruitment by soluble JAM-A has implicated JAM-A in atherogenesis ${ }^{20}$; however, owing to its pleiotropic functions the cell-specific functions of JAM-A have remained elusive. Genetic deficiency in endothelial JAM-A significantly reduced diet-induced lesion area in the thoracoabdominal aorta (Figure 1A) and aortic root (Figure 1B) of tamoxifen-treated VeCad-CreRT ${ }^{2} \mathrm{JAM}-\mathrm{A}^{\mathrm{fl} / \mathrm{fl}} \mathrm{ApoE^{-/- }}$ (eJAM- $\mathrm{A}^{-/-}$ApoE ${ }^{-/-}$) mice, as compared to their $\mathrm{Cre}^{-}\left(\mathrm{eJAM}-\mathrm{A}^{+/+} \mathrm{ApoE}^{-/}\right.$) littermates (Figure 1C, D), demonstrating a remarkable role of JAM-A on endothelial cells in promoting atherosclerosis.

As analyzed by immunohistochemistry, deficiency in endothelial JAM-A significantly decreased macrophage content, as compared to Cre controls with intact endothelial JAM-A (Figure 1E). As a hallmark for early lesion formation, we next analyzed the content of $\mathrm{CD}^{+} \mathrm{T}$ cells. Consistent with the macrophage content, mice with endothelial deficiency in JAM-A displayed a reduced content of $\mathrm{CD}^{+} \mathrm{T}$ cells (Figure 1F). Taken together, endothelial JAM-A deficiency on atherosclerotic lesion formation was associated with corresponding changes in inflammatory cell content, which likely give rise to lesion extent and composition.

\section{Redistribution and focal concentration of endothelial JAM-A in lesion-prone areas}

Previous analysis of murine atherosclerotic arteries and plaque tissue revealed an increase in JAM-A expression compared to healthy murine arteries ${ }^{19,20}$. We used ex vivo MPLSM in unfixed and intact carotid arteries of $\mathrm{ApoE}^{-/}$mice to specifically label JAM-A and to determine its distribution in plaque-prone vessels as an underlying mechanism of atherogenic leukocyte recruitment. Non-atherosclerotic tissue in the common part of the carotid artery explanted from 1 year old $\mathrm{ApoE}^{-/-}$mice on normal chow showed a mesh-like junctional pattern of JAM-A expression in luminal endothelial cells (Figure 2A, left panel). Collagen was rendered visible by SHG. In contrast, segments near the bifurcation, which are prone to atherosclerotic lesion formation due to 

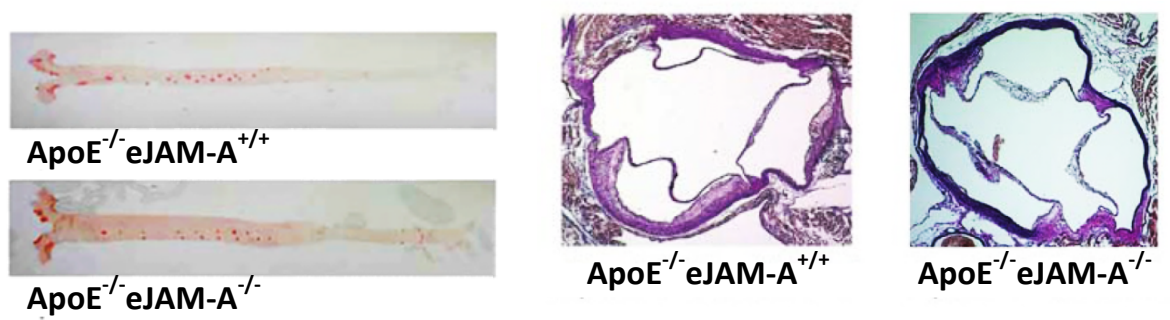

A

B
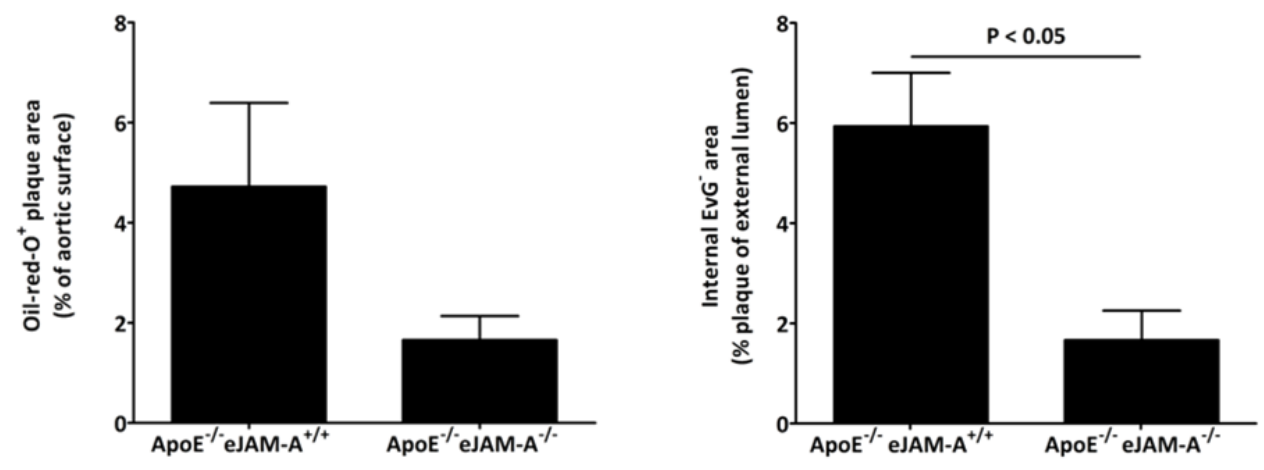

C

D
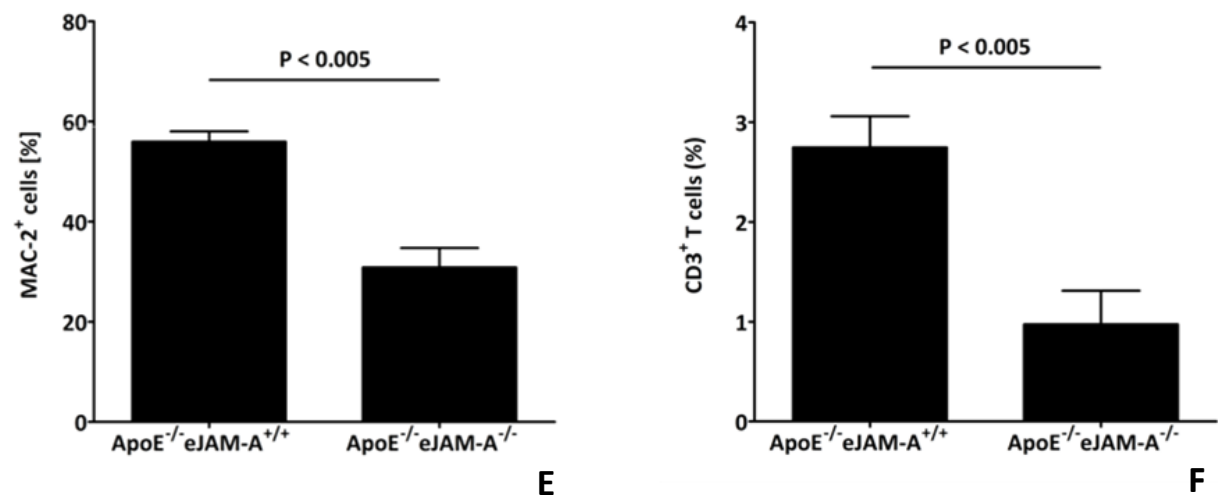

Figure 1. Endothelial JAM-A deficiency reduced plaque size and showed a concomitant reduction in macrophages and $T$ cells. Representative images of the thoracoabdominal aorta $(A)$ and the aortic root (B) of $A p o E^{-/}$eJAM- $A^{+/+}$and $A p o E^{-/}$eJAM- $A^{-/}$mice are shown. Atherosclerotic lesions of $A p o E^{-/}$eJAM- $A^{-/}$showed smaller plaques compared to $A p o E^{--}$eJAM- $A^{+/+}$mice as depicted by quantification of the $\mathrm{ORO}^{+}$area in the thoracoabdominal aorta (C), and internal EVG area in the aortic root (D). In addition, the percentage of $M A C-2^{+}$macrophages (E) and $C D 3^{+} T$ cells (F) was reduced in the aortic root lesions of ApoE ${ }^{-1}$ eJAM- $A^{-/}$mice. Data represent mean \pm SEM of individual data points depicting averaged plaque size of single slices ( $n=4$ and higher). 
disturbed flow, displayed redistribution and focal concentration of JAM-A away from endothelial cell junctions (Figure 2A, right panel). The middle panel shows the transition zone between non-atherosclerotic segments with a normal junctional JAM-A pattern (left part) and focal accumulations of JAM-A in the shoulder region of a lesion (right part). To comparatively assess the early propensity of JAM-A to focally redistribute during lesion formation, we performed co-staining with PECAM-1 (CD31) as another junctional protein expressed in endothelial cells (Figure 2B). In carotid arteries of $\mathrm{ApoE}^{-{ }^{-}}$mice fed a high-fat diet for 6 weeks, typical examples of co-staining for JAM-A and CD31 in the non-diseased common part and the plaque-prone areas near the bifurcation are shown. Using MetaMorph software, percentage of $\mathrm{JAM}-\mathrm{A}^{+} / \mathrm{CD} 31^{+}$junctions in non-diseased areas was determined (Figure $2 \mathrm{C}$ ). The bifurcation prone to nascent atherosclerotic lesion formation revealed a significant decrease in junctional co-localization of JAM-A and CD31 $(72.8 \pm 8.4 \%$ of control, $n=5$ ), implying preferential redistribution of JAM-A compared to other junctional proteins, such as CD31.

To address whether focal accumulations of endothelial JAM-A are indeed located at the luminal surface of endothelial cells or between endothelial cells and the basement membrane, we created 3D models based on z-stacks of non-diseased and plaque-prone tissues (Supplemental Figure I). Transversal sections of healthy vessels revealed continuous JAM-A expression (green) partly co-localized (yellow) with CD31 (red) stably at the level of endothelial cell nuclei (blue, right panel). In contrast, transversal sections of plaque-prone segments displayed a dotted pattern of JAM-A (green) located luminally of endothelial cell nuclei (left panel).

\section{Disturbed flow caused JAM-A redistribution}

Because the increased susceptibility to lesion formation in areas near the bifurcation is related to altered flow conditions, e.g. low or oscillatory flow, we tested whether such aberrant flow conditions as induced by partial ligation of the carotid artery could affect the localization of JAM-A. Indeed, co-staining for JAM-A and CD31 revealed a discontinuous pattern of JAM-A expression and redistribution from the junctions to the entire EC surface in the common part of partially ligated carotid arteries, similar to the pattern seen in the bifurcation of mice with established lesions (Figure 3A). This redistribution of 


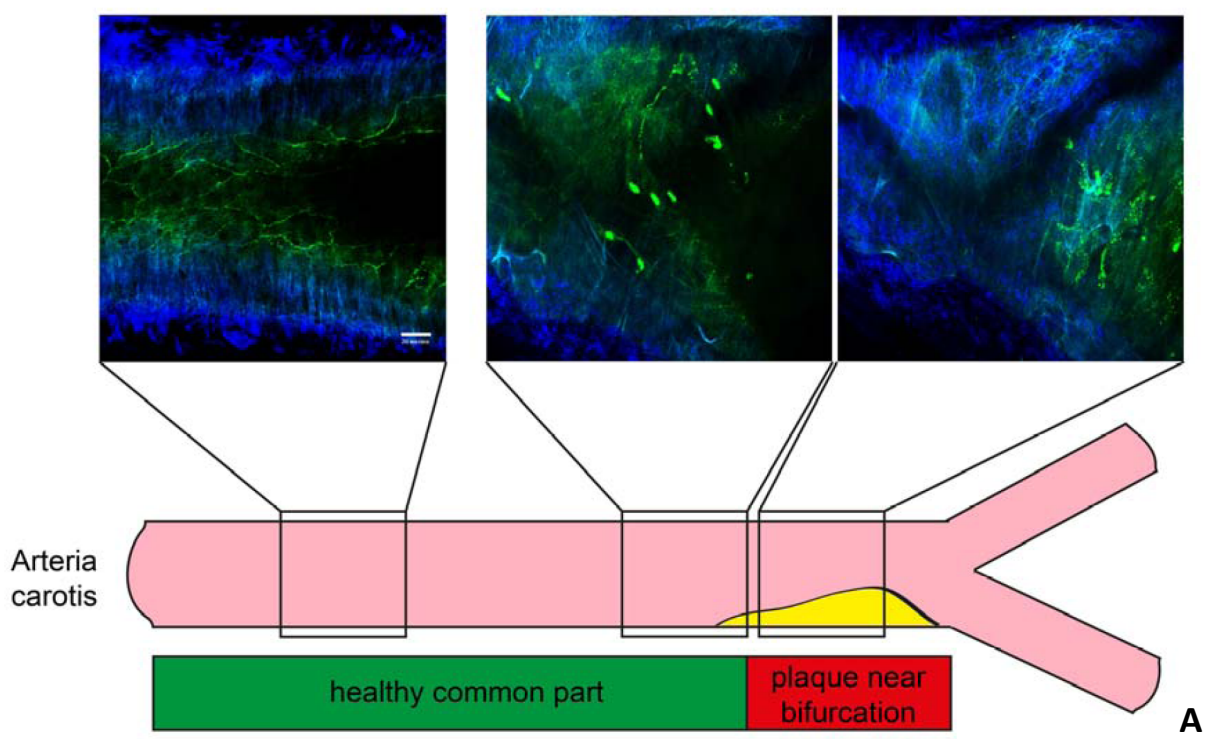

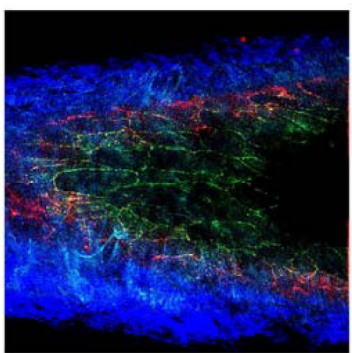

communis

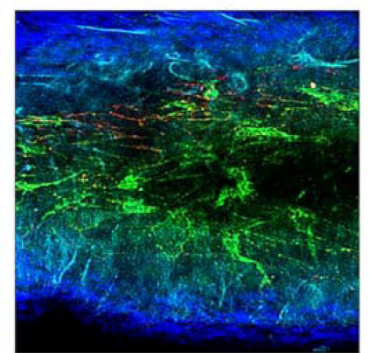

bifurcation

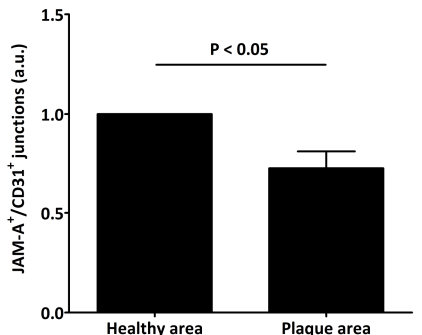

B

Figure 2. Endothelial JAM-A localized in a focal manner at the luminal site of atherosclerotic plaques. Focal dots of endothelial JAM-A (green) can be detected in the shoulder region and in the core of an atherosclerotic plaque near the bifurcation (middle and right panel) by multiphoton microscopy of ex vivo carotids mounted in a customized mounting chamber (A). A junctional JAM-A pattern could be detected in healthy tissue located in the communis of the same carotid (left panel); the carotid artery was dissected from an ApoE ${ }^{--}$mouse. Pictures are total projections of z-stack pictures. Collagen is visualized by Second Harmonic Generation (SHG). A schematic drawing of a carotid artery (lower part) depicts the site where pictures were taken. Scale bar equals $20 \mu \mathrm{m}$. Via co-staining of carotids with CD31 (red) semi-quantitative data of junctional co-localization of JAM-A and CD31 were generated. Representative pictures of the healthy area in the communis and the plaque near the bifurcation are shown (B). Scale-bar equals $20 \mu \mathrm{m}$. Semi-quantitative analysis of JAM- $A^{+} / C D 31^{+}$junctions was conducted utilizing Metamorph software (C). Measurements were conducted with ApoE ${ }^{\%}$ mice after high-fat diet feeding showing comparable plaque deposition in the carotid ( $n=5)$. Data represent mean \pm SEM. 
JAM-A was already evident after 2 weeks and was still clearly detectable within 8 weeks after partial ligation (not shown). In line with these findings, the colocalization of JAM-A with CD31 was remarkably reduced in carotids 2 and 8 weeks after partial ligation (Fig. $3 B$ ), indicating that JAM-A is preferentially redistributed from its junctional expression pattern under conditions of disturbed flow.

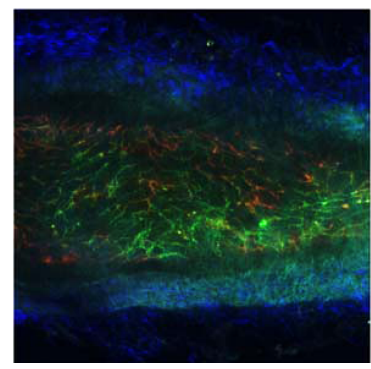

Common carotid artery

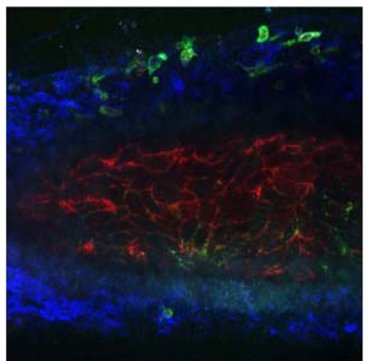

Bifurcation

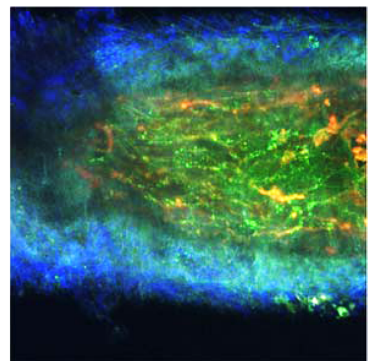

Plaque-prone bifurcation
A

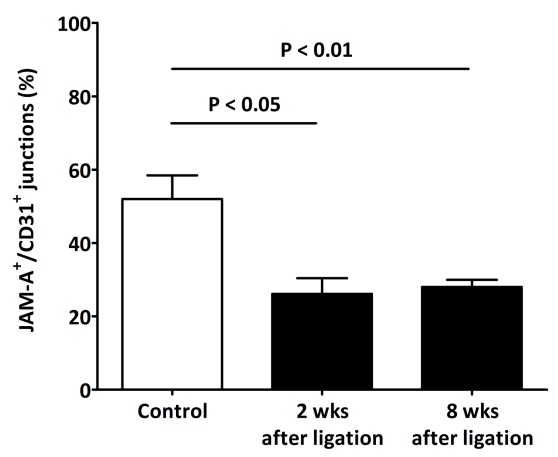

B

Figure 3. Altered shear stress caused JAM-A redistribution. Abnormal JAM-A localization (green) combined with a normal CD31 localization (red) is detectable in the common carotid artery ( $A$, left panel) and bifurcation of ligated carotid arteries without ( $A$, middle panel) and with atherosclerotic lesions ( $A$, right panel), showing that abnormal JAM-A localization occurs in the whole carotid artery after partial ligation surgery. Carotid arteries were dissected from ApoE ${ }^{-1}$ mice 2 weeks (left and middle panel) and 8 weeks (right panel) after partial ligation of the left carotid and high-fat diet. Pictures are total projections of z-stack pictures. Collagen is visible due to SHG. Scale bar equals $20 \mu \mathrm{m}$. Semi-quantitative analysis of JAM- $A^{+} / C D 31^{+}$ junctions was conducted utilizing MetaMorph software (B). Measurements were performed with ligated and unligated carotid arteries of $A p E^{-1}$ mice with subsequent high-fat diet for 2 or 8 weeks ( $n=4$ per time point), as indicated. Data represent mean \pm SEM. 
Endothelial JAM-A facilitated leukocyte transmigration in vivo and in vitro, but was not involved in vessel wall permeability

By performing in vivo and in vitro transmigration assays we took effort to validate the observed role for endothelial JAM-A in atherogenesis and the inflammatory cell recruitment into the plaques. Indeed, we found a decreased number of $\mathrm{CD} 115^{+}$monocytes in the media and adventitia of carotids of eJAM- $A^{-/-}$mice after treatment with IL-1 $\beta$, compared to eJAM- $A^{+/+}$and wild type controls (Figure 4A, 4B). Representative figures revealed that $C D 115^{+}$ monocytes (red) are abundantly present in eJAM- $A^{+/+}$controls, but that their numbers are reduced in eJAM- $A^{-/-}$mice as shown in a Z-projection derived from in situ measurements (Figure $4 \mathrm{C}$ ). Adhesion of monocytes was unaltered both in vivo and in situ (not shown).

These results were identical to results observed in in vitro assays performed with cultured ECs and primary leukocytes isolated from eJAM- $\mathrm{A}^{+/+} \mathrm{ApoE}^{-/-}$and eJAM-A $\mathrm{A}^{-/} \mathrm{ApoE}^{-/-}$mice. Time lapse experiments were performed under shear-stress conditions in which $\mathrm{CD}_{115^{+}}$monocytes transmigrated through an activated EC monolayer. Isolated mouse monocytes did not show differences in adhesion to $\mathrm{JAM}^{-\mathrm{A}^{-/}}$endothelial monolayers under shear flow conditions (Figure 4D). Less monocytes however were observed underneath the JAM-A ${ }^{-/}$EC monolayer compared to the JAM- $A^{+/+}$ECs (Figure $4 \mathrm{E})$, indicating a reduced transmigration in vitro.

To further discern whether JAM-A is involved in active transmigration, and is not involved in vascular barrier function, we determined vascular permeability in the presence or absence of endothelial JAM-A. Although leukocyte transmigration was reduced in endothelial JAM- $A^{-/}$mice, permeability for $70 \mathrm{kDa}$ (Figure 4F) and $150 \mathrm{kDa}$ dextran (not shown) was unaltered in eJAM- $\mathrm{A}^{-/} \mathrm{ApoE}^{-/}$mice on a Western type diet, indicating that ablation of JAM-A expression does not affect vascular barrier function, and that the observed transmigration of leukocytes in not a passive process. 

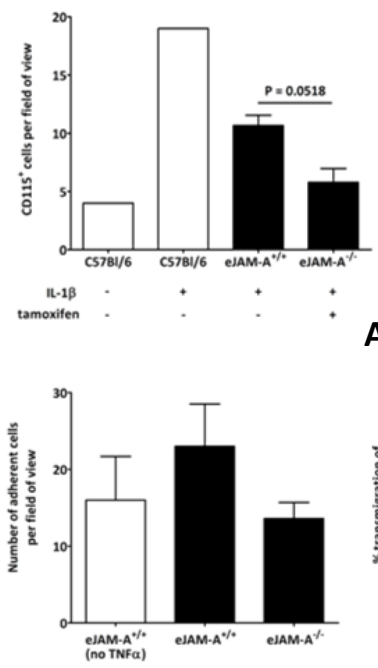

D

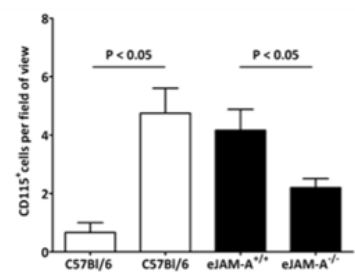

A

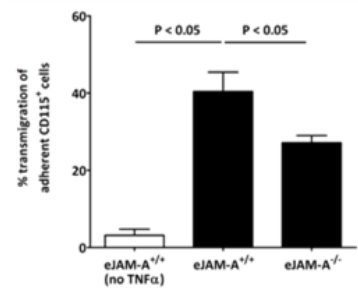

E
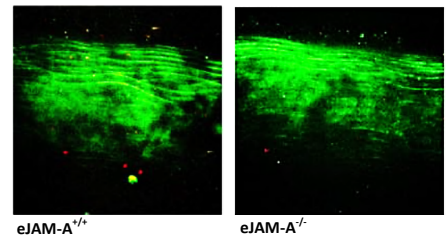

B

C

Figure 4. Deletion of endothelial JAM-A influenced leukocyte transmigration, but not leukocyte adhesion or vascular permeability. The number of cells that transmigrated into the media and adventitia was assessed after $24 \mathrm{~h}$ incubation of the common carotid artery of eJAM- $A^{-/}$mice with IL-16 administration via slowly degrading Pluronic gel. In vivo time lapses were taken subsequent to fluorescent anti-CD115 (red) and anti-CD31 (green) antibody injection via MPLSM (A), and showed a strong trend towards a decrease in transmigrated monocytes in eJAM- $A^{-1}$ mice. After in vivo imaging, mice were sacrificed and in situ z-stacks were taken directly after ventilation stopped $(B)$, and confirmed reduction of monocyte transmigration in the absence of eJAM-A. Data in $A$ and $B$ represent mean \pm SEM of individual data points depicting transmigrated cell numbers (in vivo: eJAM- $A^{+/+} n=3$, eJAM- $A^{-/} n=9$; in situ: eJAM- $A^{+/+} n=3$, eJAM- $A^{-/} n=7$ ). Representative images of the common carotid artery of eJAM- $A^{+/+}$and eJAM- $A^{-/}$mice are shown (C). Endothelial cells deficient for JAM-A were used to determine the contribution of endothelial JAM-A to leukocyte adhesion and transmigration in vitro ( $D$ and $E$ ), and showed results comparable to the in vivo and in situ data. Additionally, vascular permeability was assessed in vivo by determining fluorescence intensity of the $70 \mathrm{kDa}$ (F) and $150 \mathrm{kDa}$ (not shown) in the medial layer. Vascular permeability was not altered by eJAM-A deficiency. Data represent mean \pm SEM of at least three experiments performed independently. 


\section{Discussion}

Here, we have unraveled the impact of endothelial JAM-A deficiency on dietinduced atherosclerosis. By genetic reduction of endothelial JAM-A expression, we demonstrate that endothelial JAM-A contributed to an increase in atherosclerotic lesion formation, which was mediated by luminal redistribution of JAM-A, increasing local adhesion and transmigration of leukocytes into the atherosclerotic plaque.

The exacerbation of atherosclerotic lesion formation by endothelial JAM-A appears to be due to its redistribution from the intercellular junctions to the luminal surface, as well as to an increase in its expression under atherogenic conditions, thus increasing the availability of luminal JAM-A. Previous work has addressed redistribution of endothelial JAM-A, showing that JAM-A is re-localized to the apical surface in a cytokine-dependent manner under static and flow conditions in vitro ${ }^{14,15,24}$. Herein, we were able to extend these findings by showing a focal upregulation and clustered redistribution of JAM-A expression in endothelial cells in atherosclerotic carotid arteries, as well as after partial ligation of the healthy carotid arteries. This increase in luminal JAM-A availability likely mediates enhanced recruitment and increased content of macrophages and T cells in atherosclerotic plaques of mice, and is absent in mice with endothelial JAM-A deficiency.

Our data challenge the intuitive assumption that knocking-out a protein that is important for junctional barrier function, would cause a nonspecific influx of leukocytes and/or an increase in vascular permeability. Rather, our data refutes a role of JAM-A in limiting transendothelial migration and point towards a specific role of endothelial JAM-A in leukocyte recruitment in the context of atherosclerosis, since genetic reduction of eJAMA resulted in decreased macrophages and $T$ cells infiltration into plaques and luminal monocyte extravasation in carotid arteries in vivo. Additionally, permeability assays utilizing fluorescent dextran revealed no difference in arterial barrier function in atherosclerotic mice with endothelial JAM-A deficiency. This is in contrast to a function of JAM-A that has been described in corneal endothelial, epithelial and intestinal permeability following TNF stimulation ${ }^{25-27}$. These studies however were performed either in vitro in contrast to our ex vivo model, or in epithelial cells, not endothelial cells, making a direct comparison difficult. 
The luminal redistribution of JAM-A is facilitated by aberrant flow conditions, e.g. after partial ligation or at predilection sites of atherogenesis, whereas laminar shear flow appears to exert beneficial effects on JAM-A, limiting the susceptibility to atherogenesis. Of note, Kruppel-like factor 2 (KLF2)transduced or shear-stress-stimulated HUVECs have been found to be enriched in miR-143/145, providing a mechanism for atheroprotective communication with neighboring VSMCs via miR-145-containing microvesicles ${ }^{28}$. It would be interesting to further investigate these mechanisms, as a miR-145-dependent targeting of JAM-A has been identified in tumor cells ${ }^{29}$. miR-145 was able to suppress fascin expression and entailed a more cortical actin distribution and reduced actin stress fiber formation ${ }^{29}$. As such, it may also be responsible for limiting JAM-A redistribution, and would represent a potential target for intervention.

In relation to earlier studies, it would also be of interest to establish the role of endothelial JAM-A on microvascular endothelium, e.g. plaqueassociated vasa vasorum, which have a significant role in leukocyte recruitment to atherosclerotic plaques ${ }^{30}$. Here, we did not assess the role of eJAM-A on plaque-associated vasa vasorum. Conceivably, eJAM-A may even (in part) explain the high number of transmigrating leukocytes observed for vasa vasorum adjacent to the plaque ${ }^{30}$, as the low flow in plaque-associated vasa vasorum may implicate a redistribution of JAM-A similarly to the redistribution found at sites of disturbed flow described in this study.

Concluding, our study identifies a crucial involvement of eJAM-A in atherogenic leukocyte recruitment in response to disturbed flow at predilection sites of atherogenesis. Limiting the flow-dependent redistribution of endothelial JAM-A, e.g. by miR-145 upregulation or mimetics, may serve as a novel therapeutic approach to treat early atherosclerosis. Moreover, the focal and apical localization of endothelial JAM-A may be a suitable marker for molecular imaging of early stages of lesion formation and may be exploited as a modality for targeted delivery of immunotherapies in atherosclerosis. 


\section{Supplemental Figures}

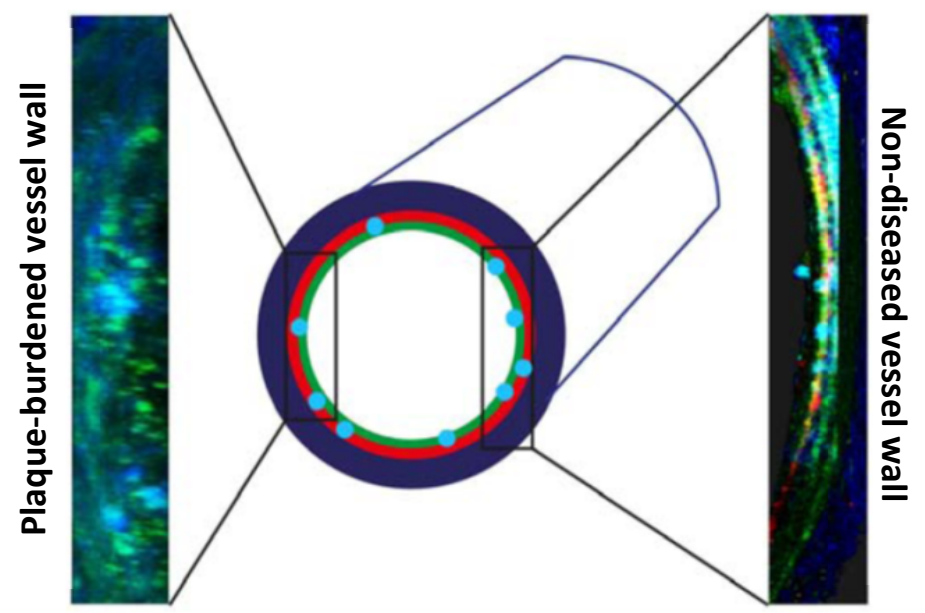

Supplemental Figure I. Differential distribution of endothelial JAM-A between the plaqueburdened and non-diseased vessel wall. In the plaque-burdened vessels wall, JAM-A (green) was distributed in a spotted pattern, more luminal from the endothelial cells (red, CD31). In contrast, in the non-diseased vessel wall, JAM-A was co-localized with the endothelial lining. Blue indicates collagen as determined by $S H G$, cell nuclei are depicted in cyan. 


\section{References}

1. Rehder D, Iden S, Nasdala I, et al. Junctional adhesion molecule-a participates in the formation of apico-basal polarity through different domains. Experimental cell research. 2006;312:3389-3403

2. Liu Y, Nusrat A, Schnell FJ, et al. Human junction adhesion molecule regulates tight junction resealing in epithelia. Journal of cell science. 2000;113 ( Pt 13):2363-2374

3. Stellos $\mathrm{K}$, Langer $\mathrm{H}, \mathrm{Gnerlich} \mathrm{S}$, et al. Junctional adhesion molecule a expressed on human cd34+ cells promotes adhesion on vascular wall and differentiation into endothelial progenitor cells. Arteriosclerosis, thrombosis, and vascular biology. 2010;30:1127-1136

4. Weber C, Fraemohs L, Dejana E. The role of junctional adhesion molecules in vascular inflammation. Nature reviews. Immunology. 2007;7:467-477

5. Bazzoni G. The jam family of junctional adhesion molecules. Current opinion in cell biology. 2003;15:525-530

6. Fraemohs L, Koenen RR, Ostermann G, et al. The functional interaction of the beta 2 integrin lymphocyte function-associated antigen-1 with junctional adhesion molecule-a is mediated by the i domain. Journal of immunology. 2004;173:6259-6264

7. Martin-Padura I, Lostaglio S, Schneemann $\mathrm{M}$, et al. Junctional adhesion molecule, a novel member of the immunoglobulin superfamily that distributes at intercellular junctions and modulates monocyte transmigration. The Journal of cell biology. 1998;142:117-127

8. Ostermann G, Weber KS, Zernecke A, et al. Jam-1 is a ligand of the beta(2) integrin Ifa-1 involved in transendothelial migration of leukocytes. Nature immunology. 2002;3:151-158

9. Cera MR, Del Prete A, Vecchi A, et al. Increased dc trafficking to lymph nodes and contact hypersensitivity in junctional adhesion molecule-a-deficient mice. The Journal of clinical investigation. 2004;114:729-738

10. Cera MR, Fabbri M, Molendini C, et al. Jam-a promotes neutrophil chemotaxis by controlling integrin internalization and recycling. Journal of cell science. 2009;122:268-277

11. Corada M, Chimenti S, Cera MR, et al. Junctional adhesion molecule-adeficient polymorphonuclear cells show reduced diapedesis in peritonitis and heart ischemia-reperfusion injury. Proceedings of the National Academy of Sciences of the United States of America. 2005;102:10634-10639

12. Woodfin A, Reichel CA, Khandoga A, et al. Jam-a mediates neutrophil transmigration in a stimulus-specific manner in vivo: Evidence for sequential roles for jam-a and pecam-1 in neutrophil transmigration. Blood. 2007;110:1848-1856

13. Ebnet K, Schulz CU, Meyer Zu Brickwedde MK, et al. Junctional adhesion molecule interacts with the $\mathrm{pdz}$ domain-containing proteins af- 6 and zo- 1 . The Journal of biological chemistry. 2000;275:27979-27988 
14. Martinez-Estrada OM, Manzi L, Tonetti $P$, et al. Opposite effects of tumor necrosis factor and soluble fibronectin on junctional adhesion molecule-a in endothelial cells. American journal of physiology. Lung cellular and molecular physiology. 2005;288:L1081-1088

15. Ozaki $\mathrm{H}$, Ishii $\mathrm{K}$, Horiuchi $\mathrm{H}$, et al. Cutting edge: Combined treatment of tnfalpha and ifn-gamma causes redistribution of junctional adhesion molecule in human endothelial cells. Journal of immunology. 1999;163:553-557

16. Mamdouh Z, Mikhailov A, Muller WA. Transcellular migration of leukocytes is mediated by the endothelial lateral border recycling compartment. The Journal of experimental medicine. 2009;206:2795-2808

17. Khandoga A, Kessler JS, Meissner $\mathrm{H}$, et al. Junctional adhesion molecule-a deficiency increases hepatic ischemia-reperfusion injury despite reduction of neutrophil transendothelial migration. Blood. 2005;106:725-733

18. Zernecke A, Liehn EA, Gao JL, et al. Deficiency in ccr5 but not ccr1 protects against neointima formation in atherosclerosis-prone mice: Involvement of il10. Blood. 2006;107:4240-4243

19. Babinska A, Azari BM, Salifu MO, et al. The $\mathrm{f} 11$ receptor (f11 $/ \mathrm{jam}-\mathrm{a}$ ) in atherothrombosis: Overexpression of $\mathrm{f} 11 \mathrm{r}$ in atherosclerotic plaques. Thrombosis and haemostasis. 2007;97:272-281

20. Ostermann G, Fraemohs L, Baltus T, et al. Involvement of jam-a in mononuclear cell recruitment on inflamed or atherosclerotic endothelium: Inhibition by soluble jam-a. Arteriosclerosis, thrombosis, and vascular biology. 2005;25:729-735

21. Bernhagen J, Krohn $\mathrm{R}$, Lue $\mathrm{H}$, et al. Mif is a noncognate ligand of cxc chemokine receptors in inflammatory and atherogenic cell recruitment. Nature medicine. 2007;13:587-596

22. Nam D, Ni CW, Rezvan A, et al. Partial carotid ligation is a model of acutely induced disturbed flow, leading to rapid endothelial dysfunction and atherosclerosis. American journal of physiology. Heart and circulatory physiology. 2009;297:H1535-1543

23. Megens RT, Oude Egbrink MG, Cleutjens JP, et al. Imaging collagen in intact viable healthy and atherosclerotic arteries using fluorescently labeled cna35 and two-photon laser scanning microscopy. Molecular imaging. 2007;6:247260

24. Shaw SK, Perkins BN, Lim YC, et al. Reduced expression of junctional adhesion molecule and platelet/endothelial cell adhesion molecule-1 (cd31) at human vascular endothelial junctions by cytokines tumor necrosis factor-alpha plus interferon-gamma does not reduce leukocyte transmigration under flow. The American journal of pathology. 2001;159:2281-2291

25. Laukoetter MG, Nava P, Lee WY, et al. Jam-a regulates permeability and inflammation in the intestine in vivo. The Journal of experimental medicine. 2007;204:3067-3076

26. Mandell KJ, Berglin L, Severson EA, et al. Expression of jam-a in the human corneal endothelium and retinal pigment epithelium: Localization and evidence for role in barrier function. Investigative ophthalmology \& visual science. 2007;48:3928-3936 
27. Vetrano $\mathrm{S}$, Rescigno $\mathrm{M}$, Cera $\mathrm{MR}$, et al. Unique role of junctional adhesion molecule-a in maintaining mucosal homeostasis in inflammatory bowel disease. Gastroenterology. 2008;135:173-184

28. Hergenreider E, Heydt S, Treguer $\mathrm{K}$, et al. Atheroprotective communication between endothelial cells and smooth muscle cells through mirnas. Nature cell biology. 2012;14:249-256

29. Gotte M, Mohr C, Koo CY, et al. Mir-145-dependent targeting of junctional adhesion molecule a and modulation of fascin expression are associated with reduced breast cancer cell motility and invasiveness. Oncogene. 2010;29:6569-6580

30. Rademakers T, Douma K, Hackeng TM, et al. Plaque-associated vasa vasorum in aged apolipoprotein e-deficient mice exhibit proatherogenic functional features in vivo. Arterioscler Thromb Vasc Biol. 2013;33:249-256 


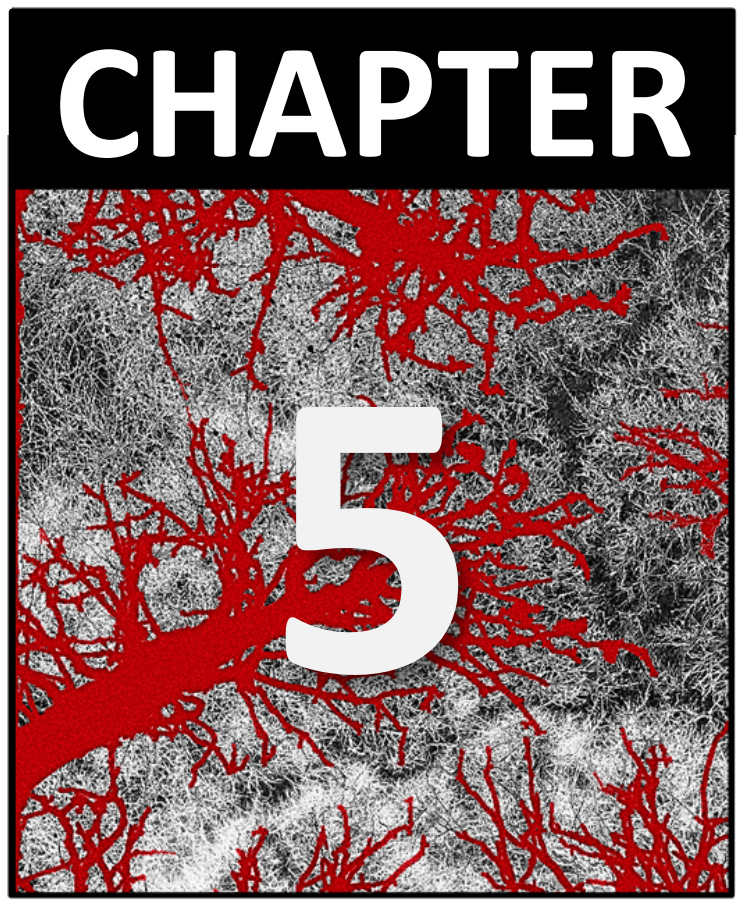




\title{
VE-cadherin as a potential mediator of microvascular permeability in atherosclerosis?
}

\author{
Timo Rademakers ${ }^{*}$, Thomas L. Theelen ${ }^{*}$, Jaap D. van Buul, \\ Jack P. Cleutjens, Pieter van de Vijver, Tilman M. Hackeng, \\ Marc A.M.J. van Zandvoort, Mat J.A.P. Daemen, and Judith C. Sluimer
}

* authors contributed equally 


\section{Abstract}

Vascular endothelial (VE)-cadherin is important for preventing leakage of plasma proteins and egress of inflammatory cells under physiologic conditions. Upon inflammation, inter-endothelial VE-cadherin dimers are disrupted, leading to a more permeable endothelium. Recently, leaky vasa vasorum (VV) were described surrounding plaque-burdened arteries in $\mathrm{ApoE}^{-/-}$mice. To elucidate the cause of the leaky plaque-associated VV, we studied whether VEcadherin disruption can explain (part of) the leaky phenotype.

Plaque-associated VV and sternohyoid microvessels (SH) were imaged in vivo in $\mathrm{LDLr}^{-/-}$mice with severe carotid atherosclerosis (52wks old, 40wks high cholesterol diet) using multiphoton laser scanning microscopy. Younger (16wks old, chow diet) LDLr ${ }^{-/}$mice served as controls. Leukocyte adhesion, transmigration, and leakage of $70 \mathrm{kDa}$ dextran were imaged with or without antibody-mediated VE-cadherin disruption. At baseline, plaque-associated VV in old $\mathrm{LDLr}^{-/-}$mice showed increased leukocyte adhesion (0.30 \pm 0.09 vs. $1.03 \pm$ 0.15 cells $\left./ \mathrm{mm}^{2} ; \mathrm{p}<0.01\right)$ and transmigration $(0.38 \pm 0.07$ vs. $2.65 \pm 0.48$ cells $\left./ \mathrm{mm}^{2} ; \mathrm{p}<0.005\right)$ compared to $\mathrm{SH}$ microvessels. After VE-cadherin blocking, SH microvessels of both young and old $\mathrm{LDLr}^{-/-}$mice showed a 2-fold increased permeability ( $p<0.05$ ), and increased leukocyte transmigration $\left(0.38 \pm 0.07\right.$ vs. $1.56 \pm 0.38$ cells $\left./ \mathrm{mm}^{2} ; \mathrm{p}<0.05\right)$ in old $\mathrm{LDLr}^{-/}$mice. In contrast, no differences in adhesion, transmigration, or microvascular permeability were found in plaque-associated VV of old LDLr ${ }^{-/}$mice after VE-cadherin blocking.

Functional testing of a VE-cadherin tandem peptide to stabilize VEcadherin in cultured HUVECs showed increased endothelial basal resistance, yet failed to prevent TNF $\alpha$-induced permeability or restoration of thrombininduced permeability. As such, the peptide was at current not a viable tool for stabilization of VE-cadherin dimerization in vivo.

While VE-cadherin blockage increased microvascular permeability in $\mathrm{SH}$ microvessels of both young and old atherosclerotic $\mathrm{LDLr}^{-/-}$mice, vascular permeability and leukocyte transmigration in plaque-associated VV were not increased beyond baseline. This suggests either lack of involvement of VEcadherin in plaque-associated $\mathrm{VV}$, or a pre-existing dysfunction or disruption of VE-cadherin. To prove a role of VE-cadherin in the leaky phenotype in plaqueassociated VV, experiments using the VE-cadherin tandem peptide, enhancing endothelial barrier function, will be crucial. 


\section{Introduction}

The inflammatory response in atherosclerosis is regarded as a key process in plaque development and progression. Endothelial cells (ECs) play an important role in this response, since the endothelial barrier regulates the entry of inflammatory cells into the atherosclerotic plaque. This is already illustrated at the onset of atherosclerosis, where dysfunction of the luminal endothelial cells is initiated by altered shear stress, causing circulating lipoproteins to accumulate in the intima. In response, luminal ECs upregulate adhesion molecules to facilitate leukocyte recruitment to the subendothelial layer. In later stages, ECs from newly formed plaque microvessels are also prominently involved in leukocyte recruitment, and are regarded to exhibit a leaky phenotype ${ }^{1,2}$. Data from human studies have already suggested that these microvessels show aberrant morphology, and electron microscopy (EM) and immunohistochemical analysis of human pathological specimens of ruptured carotid artery lesions revealed that microvascular ECs are not covered by pericytes ${ }^{3}$. Moreover, EM of microvessels from unstable human atherosclerotic lesions revealed disruptions of their inter-endothelial junctions and membrane blebbing ${ }^{3}$.

Recently, our group and others have provided in vivo evidence for a dysfunctional $\mathrm{VV}$ in atherosclerotic $\mathrm{ApoE}^{-/-}$mice ${ }^{2,4}$, in which both an enhanced leukocyte adhesion to and transmigration from the microvascular wall were shown, as well as an increase in microvascular permeability. This phenotype suggests (1) enhanced leukocyte recruitment, e.g. by upregulation of adhesion molecules like P-selectin and lymphocyte function-associated antigen-1 (LFA-1) ${ }^{4}$, and (2) potential loosening of endothelial junctions, which are of key importance for leukocyte transmigration and microvascular permeability.

Endothelial junctions consist of numerous molecules which partly share a role as junction molecule, but are simultaneously recognized by immune cells for adhesion and transmigration, e.g. junctional adhesion molecule A (JAM-A), platelet endothelial cell adhesion molecule (PECAM-1/CD31), and vascular endothelial cadherin (VE-cadherin) ${ }^{5-7}$. VEcadherin is a transmembrane protein, and has a cytoplasmic domain which binds to the actin cytoskeleton via $\alpha$-catenin and other cofactors ${ }^{8,9}$. Binding to the intercellular cytoskeleton on the one hand ${ }^{10}$, and the inter-endothelial dimerization of VE-cadherin molecules from neighboring ECs on the other 
hand, are thought to be crucial for maintaining stability and subsequently impermeability of endothelial adherent junctions ${ }^{11}$.

Permeability of the endothelial junction is achieved by phosphorylation of the cytoplasmic VE-cadherin residues. Upon phosphorylation, VE-cadherin dissociates from its co-factors, disconnects from the actin cytoskeleton, and is internalized. In contrast, de-phosphorylation by VE-protein tyrosine phosphatase (VE-PTP) ${ }^{11-14}$ prevents the disruption of VE-cadherin complexes and VE-cadherin internalization.

VE-cadherin has been identified as the main mediator of permeability, and blocking of VE-cadherin dimerization has been shown to increase fluidic permeability ${ }^{9}$. Furthermore, several studies have shown an additional role of VE-cadherin in leukocyte extravasation ${ }^{15-17}$. Mice treated with a VE-cadherin blocking antibody displayed a $70 \%$ increased neutrophil extravasation in a peritonitis model ${ }^{15}$. These data on the effects of VE-cadherin, in combination with previous data from our group ${ }^{2}$ led to the hypothesis that VE-cadherin may be involved in microvascular leakage in atherosclerosis, and that a VEcadherin-targeted intervention reduces microvascular leakage in plaque associated microvessels.

Stabilization of the inter-endothelial VE-cadherin complex has been described in vivo by either use of a specific protein linker (tandem peptide) ${ }^{18}$ or by using transgenic mouse models ${ }^{14,19}$. The VE-cadherin protein linker is a peptide that links VE-cadherin molecules of two ECs leading to a more impermeable endothelial junction. Its application resulted in a 5-fold reduction of permeability in healthy hind limb vessels of rats challenged with tumor necrosis factor- $\alpha(\mathrm{TNF} \alpha)^{18}$.

Here, we investigated the effects of this VE-cadherin-linking tandem peptide to stabilize inter-endothelial junctions in vitro. In addition, we studied the effects of VE-cadherin disruption by a VE-cadherin blocking antibody to identify the role of VE-cadherin in the leaky phenotype in atherosclerotic plaque-associated vasa vasorum. To this end, we applied multiphoton laser scanning microscopy (MPLSM) to quantify microvascular permeability and leukocyte adhesion and transmigration in an in vivo setting. 


\section{Methods}

\section{VE-cadherin stabilization}

\section{Tandem peptide production}

VE-cadherin tandem peptide was produced by solid phase peptide synthesis (using Boc protecting groups) by the Department of Biochemistry, Maastricht University similar to previous publications ${ }^{20}$. The peptide was fluorescently labeled with Oregon Green (OG488). Synthesized peptide was purified by high pressure liquid chromatography, identified using mass spectrometry, and was lyophilized before storage at $-80^{\circ} \mathrm{C}$.

\section{Testing of tandem peptide in vitro}

In vitro testing of the tandem peptide was performed at Sanquin, Amsterdam. The cellular location of the tandem peptide was detected by visualization of the OG488 label with confocal microscopy of human umbilical vein endothelial cells (HUVECs) confluent monolayers. HUVECs were treated with $20 \mu \mathrm{M}$ tandem peptide and challenged with human tumor necrosis factor- $\alpha$ (TNF $\alpha$ ) prior to imaging. For better orientation, co-staining with an Alexa647-labeled VE-cadherin antibody (BD Pharmingen; $0.5 \mathrm{mg} / \mathrm{ml}$ stock) was performed.

Electric Cell-substrate Impedance Sensing (ECIS) was used to test permeability reducing properties of the tandem peptide, comparable to previous experiments ${ }^{21}$. Shortly, a confluent monolayer of HUVEC was grown on fibronectin coated electrode arrays. Cells were incubated with $20 \mu \mathrm{M}$ tandem peptide or vehicle control when basal electrical resistance was reached. Short ( 8 hours) and long term (60 hours) changes in electrical resistance after challenge with human TNF $\alpha$ and after thrombin-induced permeability were measured on the ECIS-Model-100 Controller (BioPhysics). 


\section{VE-cadherin blocking}

\section{Animals}

For in vivo experiments, 12-weeks old male $\mathrm{LDLr}^{-/-}$mice were placed on a highcholesterol diet $(0.25 \%$ cholesterol, Special Diet Services) for 40 weeks $(n=18)$ Young male $\mathrm{LDLr}^{-/}$mice (16 weeks of age, normal chow; $\mathrm{n}=10$ ) served as controls. Male C57BI/6 mice (12 weeks of age, normal chow; $n=14$ ) served as donors for isolation of bone marrow derived cells from the tibia and femur. Mice were supplied by the institutional animal facility, and were housed in conventional cages. Experiments were approved by the local animal ethics committee and performed according to institutional guidelines.

Four hours before in vivo imaging, mice were injected with $60 \mu \mathrm{l}$ VEcadherin blocking antibody ${ }^{15}$ (BD Pharmingen; $0.5 \mathrm{mg} / \mathrm{ml}$ stock) or control antibody ( $n=9 /$ group for aged LDLr ${ }^{-/-}$mice, $n=5 /$ group for young LDLr ${ }^{-/}$mice), $125 \mu \mathrm{l} 70$ kDa dextran-tetramethylrhodamin (TMR; Invitrogen; $3 \mathrm{mg} / \mathrm{ml}$ stock), and $100 \mu \mathrm{l}$ phosphate-buffered saline (PBS) intravenously via the tail vein. Mice then received an additional intravenous injection of $80 \mu$ CD31-FITC (BD Pharmingen; $0.5 \mathrm{mg} / \mathrm{ml}$ stock) and $1 \times 10^{7}$ Syto44-labeled bone marrow derived cells in $200 \mu \mathrm{l}$ PBS 30 minutes prior to imaging. Bone marrow derived cells were isolated from the tibia and femur from $\mathrm{C} 57 \mathrm{Bl} / 6$ control mice, labeled with Syto44 $5 \mu \mathrm{M}$ (Invitrogen) for $30 \mathrm{~min}$ at $37^{\circ} \mathrm{C}$, washed and kept on ice until further use.

Mice were anaesthetized using a dorsal subcutaneous injection with ketamine $(100 \mathrm{mg} / \mathrm{kg}$ body weight, Nematek) and xylazine $(10 \mathrm{mg} / \mathrm{kg}$ body weight, Sedamun) through a catheter made from a $28 \mathrm{~mm}$ polyethylene tube fitted with a $30 \mathrm{G}$ needle. Body temperature was kept at $37^{\circ} \mathrm{C}$ by means of a heating pad. Every 30 minutes an additional dose of anaesthetics was administered to maintain anaesthesia. The carotid artery and the sternoyhoid muscle were exposed, and mice were imaged in vivo for up to 2 hours. After in vivo imaging, mice were sacrificed by intraperitoneal pentobarbital injection and in situ imaging was performed for 3D tissue analysis. 


\section{Multiphoton laser scanning microscopy}

MPLSM imaging was performed on a Leica SP5 imaging platform (Leica Microsystems, Wetzlar, Germany) that integrates two photon microscopy with fast resonant scanning, and uses a Compact Ultrafast Ti:Sapphire Laser (Chameleon, Coherent, USA). An excitation wavelength of $880 \mathrm{~nm}$ was used in all experiments. Tissues were observed using a 20x water-dipping objective (HCX PL APO L 20x 1.0 water immersion objective, Leica Microsystems, Germany) with a numerical aperture of 1.00 and an integrated optical zoom mechanism allowing total magnification up to 60x. Photo-multiplier tubes (PMTs) were used to detect four spectral regions: $390-410 \mathrm{~nm}$ (SHG for imaging collagen fibrils), $420-470 \mathrm{~nm}$ (Syto44 for cell nuclei), $515-560 \mathrm{~nm}$ (FITC) and 570-600 $\mathrm{nm}$ (TMR).

In vivo MPLSM imaging was performed at a frame size of $400 \times 400$ pixels (pixel size: $1.0882 \times 1.0882 \mu \mathrm{m}$ ), scanning at $8000 \mathrm{~Hz}$ for fast recording (20 frames/sec) and using a line average of 2 (10 frames/sec) for reduced noise scans. Per time series, a total of 500 frames was recorded. Field of view at 20x magnification is $434.2 \times 434.2 \mu \mathrm{m}$. After in vivo imaging, the animal was sacrificed and additional in situ imaging performed to examine the carotid artery at higher resolution and an increased signal-to-noise ratio without the motion artifacts. In situ imaging was performed at a frame size of $512 \times 512$ pixels (pixel size: $0.847 \times 0.847 \mu \mathrm{m}$ ), scanning at $250 \mathrm{~Hz}$ and an interplanar distance of $1.00 \mu \mathrm{m}$.

\section{Data analysis}

All data analysis was performed using ImageJ (http://imagej.nih.gov/ij/). 3D rendering was performed using an ImageJ plugin for high level 3D visualization ${ }^{22}$. Microvessel diameter was assessed by measuring the maximum distance between the opposite sides of the microvessel wall at ten separate points per microvessel, from which the median diameter per microvessel was calculated.

Leukocyte adhesion to the vessel wall was assessed by in vivo tracking of Syto44-labeled bone marrow derived cells that remained at the same point within the vessels for at least 250 frames. Adhesion and transmigration rates were normalized to the microvessel surface, which was derived from the microvessel length and microvessel diameter. The adjacent sternohyoid muscle capillaries served as control microvessels. Adhesion and transmigration 


\section{CHAPTER 5}

was assessed for all microvessels in 10 frames for plaque-associated vasa vasorum and in 5 frames for sternohyoid microvessels.

Microvascular permeability was determined by calculating the ratio of the extraluminal/intraluminal fluorescence intensity of the 70 kDa dextranTMR, and corrected for intrinsic autofluorescence present in the tissue. Extraluminal tissue was defined as the area within $1.5 x$ vessel diameter's distance of the microvessel wall. Microvascular permeability was assessed in all microvessels in 10 frames for plaque-associated vasa vasorum and in 5 frames for control sternohyoid microvessels.

Mice were excluded from analysis in case of local bleeding during preparation or imaging (2/18 for atherosclerotic $\mathrm{LDLr}^{-/-} ; 1 / 10$ for non-diseased $\mathrm{LDLr}^{--}$), or in absence of in vivo imaging data due to early death before imaging (2/18 for atherosclerotic LDLr $\left.{ }^{-/}\right)$.

\section{Histology and fluorescence microscopy}

Subsequent to in situ imaging, mice were perfused with PBS and $1 \%$ paraformaldehyde, and carotid arteries and kidneys were isolated for histology, paraffin-embedded and cut into serial sections. Selected sections proximal to the bifurcation were stained in $0.3 \%$ Sudan black solution (in $70 \%$ ethanol, Sudan Black B) to reduce auto-fluorescence and pictures were made using a Leica DM5000b fluorescence microscope (Leica Microsystems) and analyzed with Leica Qwin (Leica Microsystems). The area of the intima, media and adventitia were defined by the autofluorescence in the OG488 channel (green) and the TMR positive area was detected by means of the TMR positive area in the N21 channel (red) (Supplemental Figure I). Adjacent paraffinembedded sections were stained immunohistochemically (IHC) for CD31 (BD Bioscience) to confirm MPLSM data on vasa vasorum presence.

\section{Statistics}

Data are presented as mean \pm SEM, unless stated otherwise. The nonparametric Mann-Whitney $U$ test was used for statistical analysis. Results were considered statistically different when $p<0.05$. 


\section{Results}

Old, WTD-fed LDLr/- mice showed plaque-associated vasa vasorum

First, we assessed presence and functionality of plaque-associated vasa vasorum in 52 weeks old atherosclerotic $\mathrm{LDLr}^{-/-}$mice and their younger 16 weeks old controls. IHC staining for CD31 showed a clear presence of CD31 ${ }^{+}$ plaque-associated VV in old, WTD-fed LDLr ${ }^{-/}$mice (arrows, Figure 1A), but not in the adventitia of young $\mathrm{LDLr}^{-/-}$mice (Figure 1B). MPLSM confirmed that all old $\mathrm{LDLr}^{-/-}$mice exhibited a plaque-associated VV (Figure 1C), whereas those were absent in young $\mathrm{LDLr}^{-/-}$mice (Figure 1D). Median VV diameters were 15.5 and $7.1 \mu \mathrm{m}$ for $1^{\text {st }}$ and $2^{\text {nd }}$ order microvessels. Moreover, plaque-associated VV showed an increased baseline adhesion and transmigration compared to $\mathrm{SH}$ microvessels, whereas microvascular permeability was not increased (Figure 2).
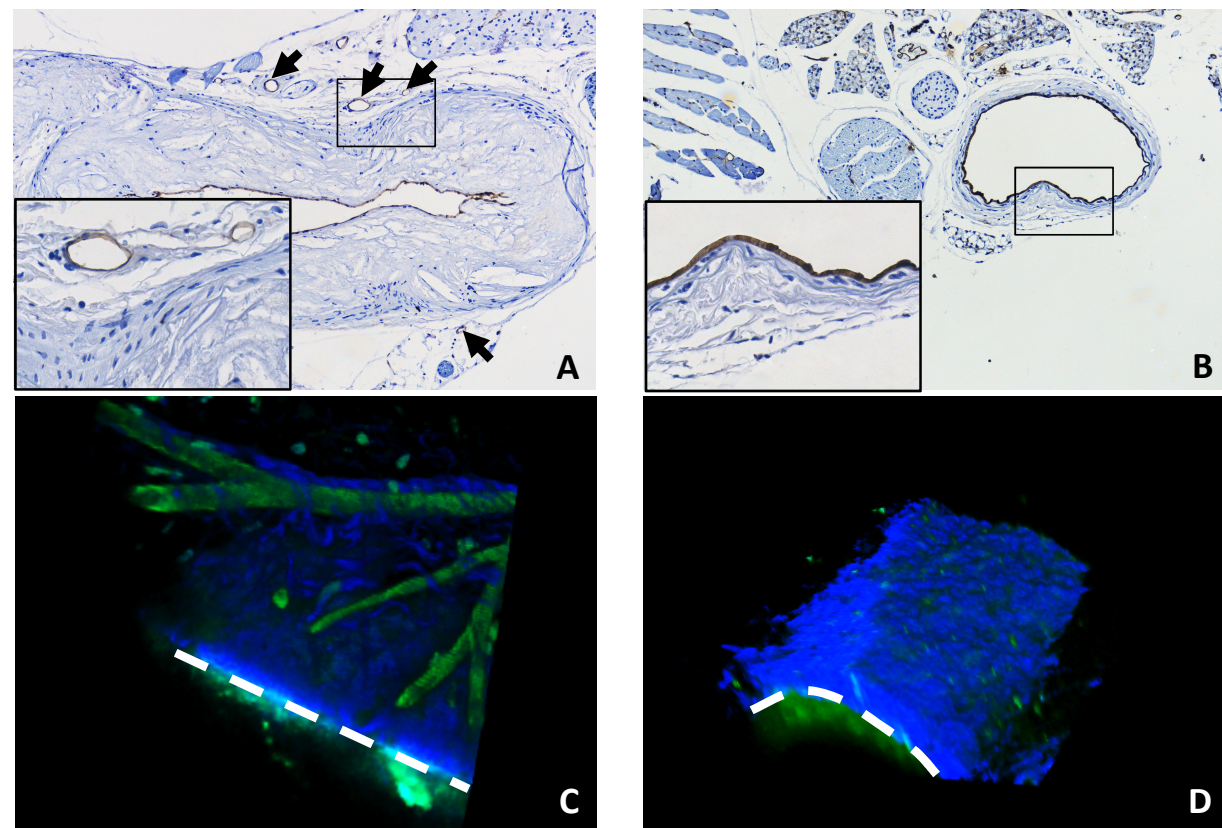

Figure 1. Plaque-associated VV in $\mathbf{5 2}$ weeks old $\mathbf{L D L r ^ { - / }}$ mice. IHC staining revealed microvessels in the adventitia (arrows) in old $L D L r^{-1-}$ mice ( $A$; 10x magnification, inset: 40x magnification), but not in young $L D L r^{-/}$mice ( $B$; 10x magnification, inset: 40x magnification). MPLSM also showed that plaque-associated VV (green) were present in the collagen-rich (blue) adventitia in old $L D L r^{-/}$mice $(C)$, yet not in young $L D L r^{--}$mice $(D)$. Dotted line indicate outline of the arterial wall. 

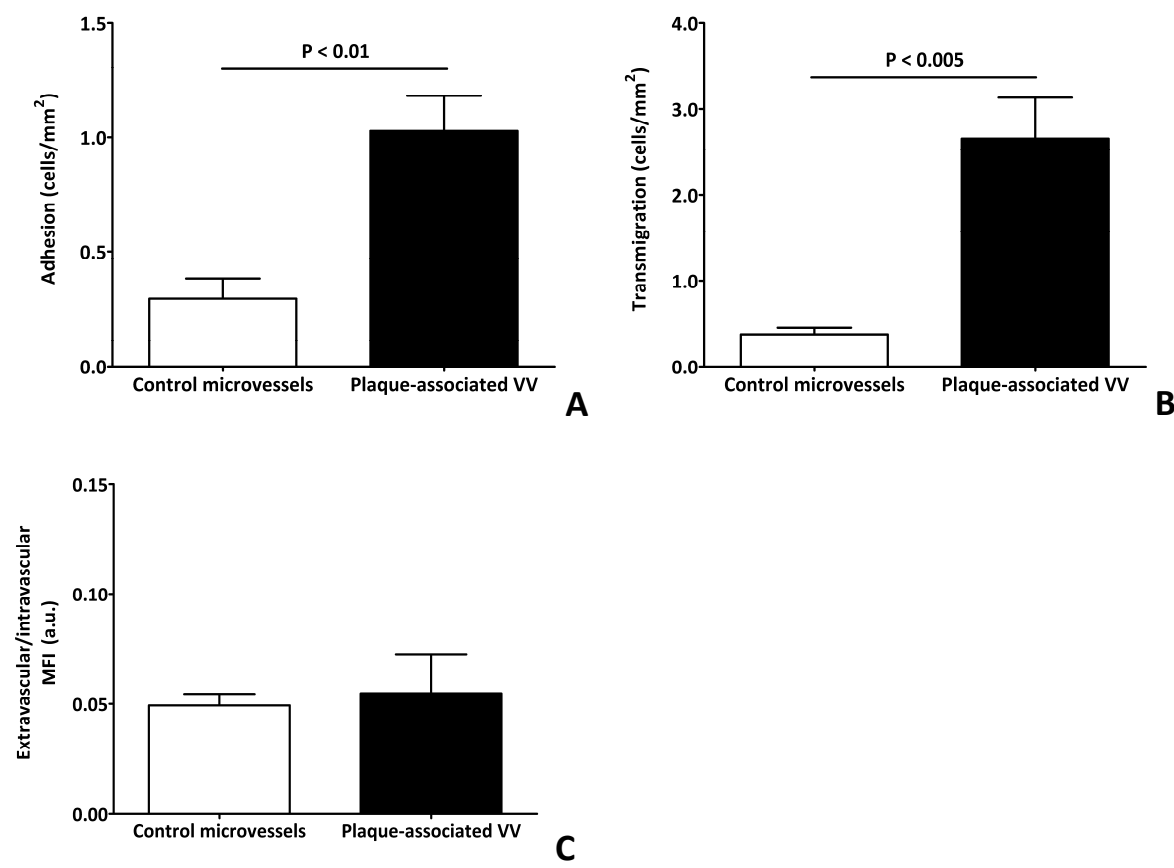

Figure 2. Baseline characteristics of $\mathrm{SH}$ (control) microvessels and plaque-associated VV. In old $\mathrm{LDLr}^{-/-}$mice, leukocyte adhesion (A) and transmigration (B) at baseline were increased compared to SH microvessels of similar diameter. Microvascular permeability (C) was not significantly different.

VE-cadherin tandem peptide was able to modestly stabilize VE-cadherin, yet did not prevent TNF $\alpha$-induced permeability in vitro

The VE-cadherin protein linker (tandem peptide) was clearly distributed along the endothelial junctions of HUVECs (Supplemental Figure II). Functional testing revealed that the tandem peptide increased endothelial resistance at short and long term (Figure 3A and $3 B$ ). The increase in endothelial resistance, although significant, was modest (Figure 3B). Remarkably, the tandem peptide failed to prevent TNF $\alpha$-induced permeability as well as restoration of thrombin-induced permeability (Figure $3 \mathrm{C}$ ). As such, the peptide was not a viable tool for stabilization of VE-cadherin dimerization in vivo. 
VE-cadherin blocking increased leukocyte transmigration and microvascular permeability in sternohyoid microvasculature in vivo

To assess efficacy of the VE-cadherin blocking antibody, we studied leukocyte adhesion, transmigration, and microvascular permeability in microvessels of the sternohyoid muscle as described previously ${ }^{2}$. VE-cadherin blocking antibody did not alter microvessel diameter (Supplemental Figure III-A). Both in young and old $\mathrm{LDLr}^{-/}$mice, we observed no differences in leukocyte adhesion upon VE-cadherin blocking (Figure 4A, 4B), while leukocyte transmigration and microvascular permeability for $70 \mathrm{kDa}$ dextran-TMR were increased in old LDLr-- mice (Figure 4C-4F; Supplemental Figure IV).

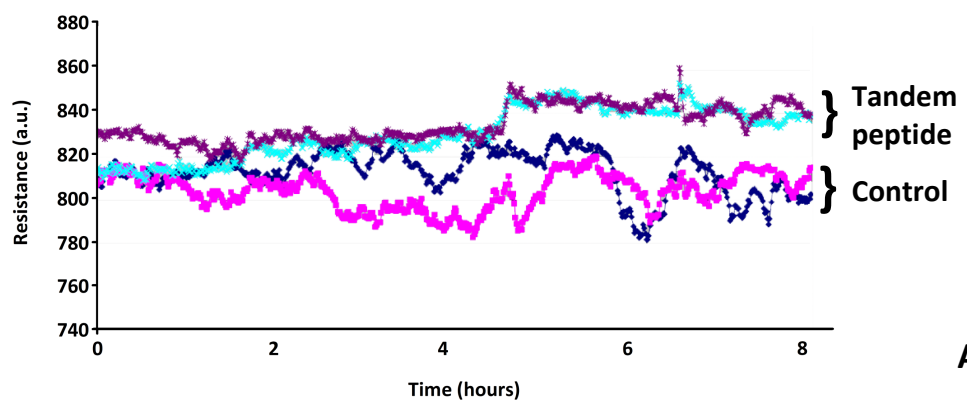

A
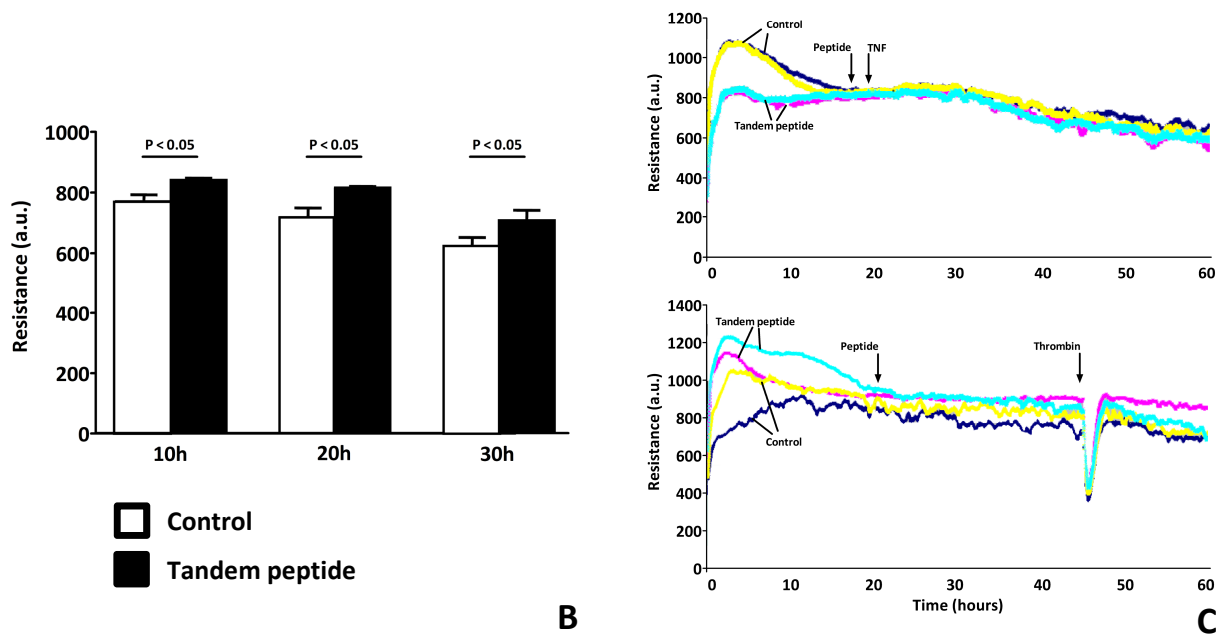

Figure 3. VE-cadherin tandem peptide induced increased endothelial barrier function, but did not prevent TNFa-induced permeability or restoration of thrombin-induced permeability. Resistance measurements of HUVECS grown in monolayer were performed using ECIS. Tandem peptide was added to endothelial cells when baseline resistance was reached ( $A$, time point 0$)$. Follow-up of vascular resistance with time revealed an increased resistance for up to 30 hours after addition of the tandem peptide (B). Tandem peptide could not prevent TNFa-induced permeability ( $C$, upper panel) or restoration of thrombin-induced permeability ( $C$, lower panel). 


\section{CHAPTER 5}
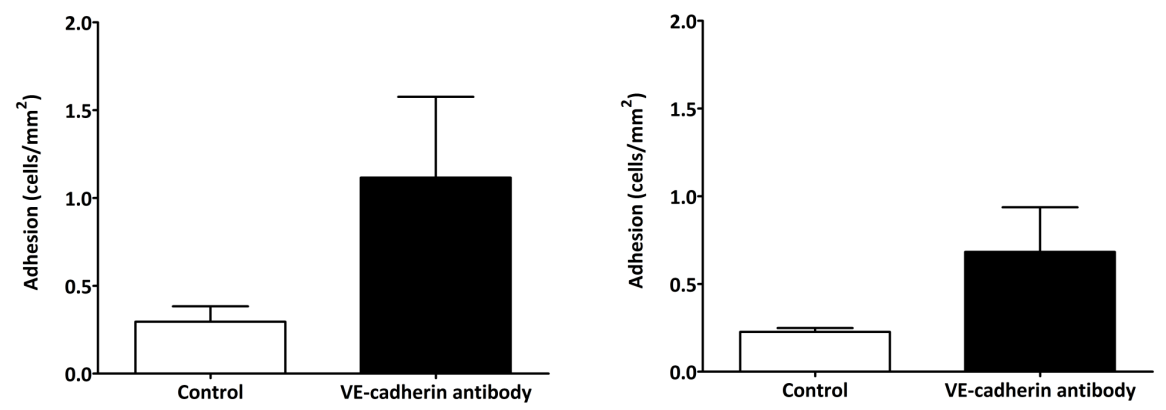

A
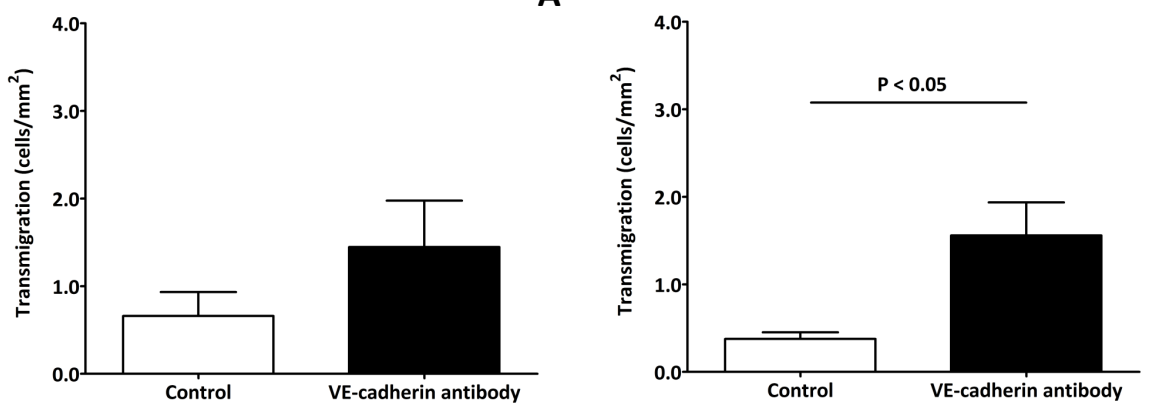

C
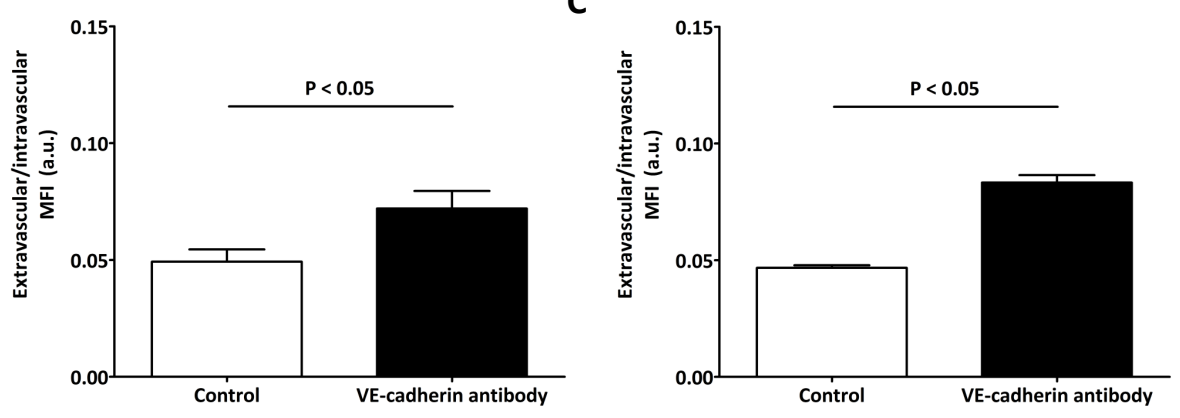

E

Figure 4. Leukocyte transmigration and microvascular permeability in SH microvessels in old $\mathrm{LDLr}^{-/}$mice was enhanced after VE-cadherin blocking antibody treatment. Adhesion of leukocytes is unaffected by VE-cadherin blockage both in young $(A, p=0.12)$ and old $L D L r^{-/}$mice $(B, p=0.15)$. In contrast, leukocyte transmigration is increased after treatment in young $(C$, $p=0.24)$ and old $L D L r^{-/}$mice $(D, p<0.05)$. In addition, microvascular permeability is significantly increased in young (E) and old $(F) L D L r^{-/-}$mice ( $n=4 /$ group for young $L D L r^{-/}$mice; $n=7-8 /$ group for old $L D L r^{-/}$mice). 
No significant differences in baseline adhesion, transmigration and microvascular permeability were observed between microvessels of young, chow-fed and old, WTD-fed LDLr-/- mice in this sternohyoid microvascular bed.

\section{VE-cadherin blocking did not alter leukocyte adhesion, transmigration and} microvascular permeability in plaque-associated vasa vasorum in vivo Given the efficacy of the VE-cadherin blocking antibody to increase permeability and transmigration in $\mathrm{SH}$ microvessels, we also investigated the effect of the VE-cadherin blocking antibody on plaque-associated VV. VEcadherin blocking antibody did not alter microvessel diameter (Supplemental Figure (II-B), and microvascular permeability was not increased at baseline (Figure $2 \mathrm{C}$ ). Similar to the $\mathrm{SH}$ microvessels, the antibody did not affect leukocyte adhesion (Figure 5A). In contrast to the SH microvasculature, we did
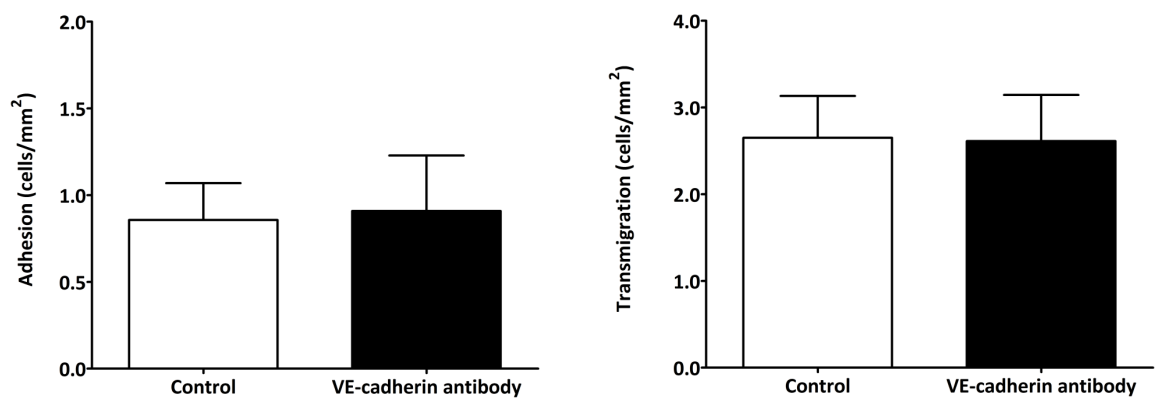

A

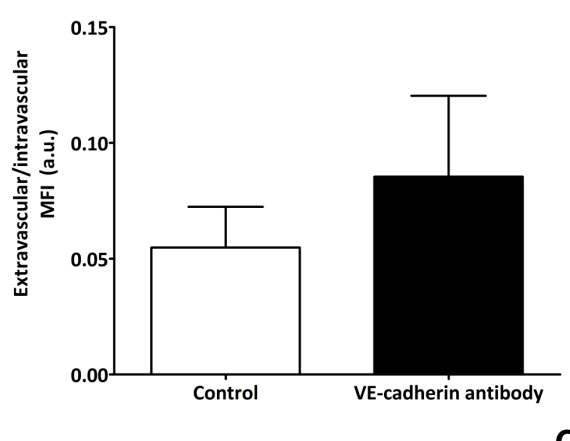

C

Figure 5. VE-cadherin blocking antibody did not exacerbate the pre-existing leaky phenotype of plaque-associated $V V$. Plaque-associated $V V$ in old $L D L r^{-/}$mice show a leaky phenotype with increased leukocyte adhesion, transmigration and microvascular permeability (A-C). Blocking VEcadherin dimerization did not further increase leukocyte adhesion (A), transmigration (B) or permeability $(C)$ in vivo. 


\section{CHAPTER 5}

not observe any changes in leukocyte transmigration (Figure 5B; Supplemental Figure V) or microvascular permeability (Figure 5C) after VE-cadherin blocking antibody treatment.

Histological analysis showed an increase in 70 kDa dextran-TMR within the atherosclerotic lesions, confirming the efficacy of the VE-cadherin antibody on carotid luminal endothelium (Figure 6A). Yet, no increased $\mathrm{TMR}^{+}$area was found in the media or the adventitia. Interestingly, in young $\mathrm{LDLr}^{-/}$mice, a high baseline $\mathrm{TMR}^{+}$area was observed, which increased after VE-cadherin blocking antibody treatment.
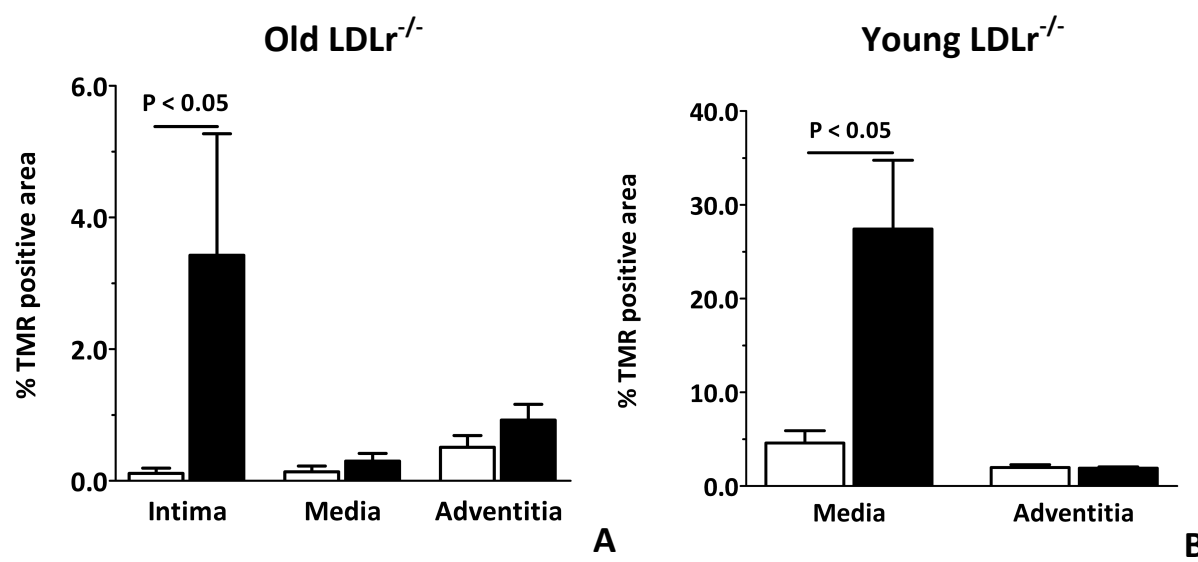

Figure 6. Efficacy of VE-cadherin blocking antibody was confirmed by histological analysis. Dextran-TMR positive area was determined in the atherosclerotic plaque (intima), media and adventitia of old $L D L r^{-/}$mice (A). VE-cadherin blocking antibody treatment (black bars) led to an increase in intimal dextran-TMR compared to controls (white bars). Medial and adventitial dextran-TMR was unaltered. In young $L D L r^{--}$mice, a high $T M R^{+}$area was observed in the media, and was significantly increased after VE-cadherin blocking antibody treatment (B). Adventitial dextran-TMR was unaltered. 


\section{Discussion}

Here, we studied the function of the junctional protein VE-cadherin in plaqueassociated vasa vasorum. Although a leaky phenotype was present in control microvessels after VE-cadherin blocking, the addition of the VE-cadherin blocking antibody did not cause an additional increase in leukocyte transmigration or microvascular permeability in plaque-associated vasa vasorum, suggesting that (i) VE-cadherin function may already be impaired in plaque-associated vasa vasorum or (ii) VE-cadherin is not involved in the leaky phenotype in plaque-associated vasa vasorum.

The observed effects of the VE-cadherin blocking antibody in both control microvessels of old, WTD-fed $\mathrm{LDLr}^{-/}$mice and in normal chow fed younger $\mathrm{LDLr}^{-/}$mice are in line with previous findings that VE-cadherin is involved both in leukocyte transmigration and vascular permeability ${ }^{15}$. In the sternohyoid microvessels, leukocyte transmigration was two to four times higher after VE-cadherin functional blocking, while microvascular permeability was increased by $50-70 \%$. Leukocyte adhesion was not affected by VE-cadherin functional blocking.

Efficacy of the VE-cadherin blocking antibody could also be confirmed by histological analysis, where we were able to detect a significant increase in $70 \mathrm{kDa}$ dextran-TMR within the atherosclerotic lesion, but not in the media or the adventitia. The luminal endothelium will also be affected by VE-cadherin blocking, and a concomitant increase in permeability will cause a rise in dextran-TMR in the underlying tissue, e.g. the atherosclerotic plaque. In young $\mathrm{LDLr}^{-/}$mice we observed a significant increase in dextran-TMR in the medial layer, which was not present in the old $\mathrm{LDLr}^{-/-}$mice. The high baseline $\mathrm{TMR}^{+}$ area in the media and adventitia of young $\mathrm{LDLr}^{-/-}$mice were most likely due to diffusion of the probe over the endothelial layer, also at baseline. In the old $\mathrm{LDLr}^{-/-}$mice, the dextran-TMR leaked into the plaque itself, and did not reach the medial or adventitial layer. Taken together, the luminal endothelium may also influence fluorescence intensities adventitia. In the MPLSM study, we therefore stringently measured fluorescent intensities directly adjacent to the plaque-associated vasa vasorum, and corrected for background fluorescence which may be present as a result of luminal permeability. As such, it was possible to assess the local role of VE-cadherin, here in plaque-associated vasa vasorum. This approach is more sensitive and specific compared to the 
histological approach, which only is a measure of total vascular permeability, and not vascular permeability of the plaque-associated vasa vasorum. By confining histological analysis to the area around the plaque-associated vasa vasorum, it might be possible to also assess microvascular permeability by histology, although the sensitivity of this method remains unclear.

Old, WTD-fed LDLr ${ }^{-/}$showed a similar plaque-associated vasa vasorum as previously described in $\mathrm{ApoE}^{-/-}$mice. Microvessel diameters and parameters like leukocyte adhesion and transmigration levels were comparable ${ }^{2}$. Recruitment numbers of leukocytes were slightly higher in the $\mathrm{ApoE}^{-/-}$model, which is in line with previous observations of heightened systemic inflammation compared to $\mathrm{LDLr}^{-/-}$mice ${ }^{23}$. Although we could clearly observe a VE-cadherin-dependent increase in transmigration in control microvessels, and higher baseline transmigration in the plaque-associated vasa vasorum, it would be of interest to challenge the local microvasculature to determine if the inflammatory response and microvascular permeability were maximal or submaximal. Stimulation with histamine or TNF ${ }^{18}$ would yield insight into whether maximal permeability was indeed already achieved. If leukocyte transmigration and/or permeability could be increased, this would indicate that the inter-endothelial junctions can be disrupted beyond VE-cadherin blocking, suggestive of a limited or absent role for VE-cadherin in microvascular permeability in plaque-associated vasa vasorum.

More importantly, it would be of great interest to assess the effect of VE-cadherin blocking antibody treatment on the plaque-associated vasa vasorum ultrastructurally using electron microscopy, and thus evaluate whether open inter-endothelial junctions can explain the increased leukocyte transmigration and microvascular permeability. A clear dissociation of neighboring microvascular ECs by blocking of VE-cadherin would thus explain the increased microvascular permeability by structural defects. Interestingly however, previous studies have shown that loss of VE-cadherin only resulted in minor ultrastructural changes in the endothelial junctions, although an increase in vascular permeability was found ${ }^{24,25}$. Data from our group also suggested no aberrant endothelial junctions in plaque-associated vasa vasorum of aged $\mathrm{ApoE}^{-/-}$mice ${ }^{2}$, nor in $\mathrm{LDLr}^{-/-}$mice at different time points of WTD (unpublished observations). This would suggest a functional and not a structural defect in VE-cadherin to be causative for increased vascular 
permeability, and would shed a new light on the role of VE-cadherin as a junctional molecule.

To however prove that dysfunction or potential downregulation ${ }^{26,27}$ of VE-cadherin has a role in the leaky phenotype observed in plaque-associated microvessels, the experiments using the VE-cadherin tandem peptide in vivo to enhance endothelial barrier function are crucial. Not only will those experiments prove whether VE-cadherin is causal in microvascular permeability in plaque-associated vasa vasorum, but it may also confer a potential future target for interventions.

Unfortunately, study of the tandem peptide and its effect on microvascular permeability and leukocyte recruitment in vivo were not yet possible. The in vitro results of the VE-cadherin tandem peptide only showed a very limited effect on endothelial barrier function, and were in strong contrast to previous work from Heupel et al, who described a dramatic increase in resistance by addition of VE-cadherin tandem peptide in vitro and in vivo ${ }^{18}$. Optimization of experimental conditions and/or improving the efficacy of the tandem peptide will be required before in vivo experiments are viable. Alternatively, an inducible VE-cadherin-VE-PTP complex mouse model would provide an interesting model to investigate the role of microvascular VEcadherin in atherosclerosis ${ }^{14}$. In such a model, it will be possible to study the long term effects of VE-cadherin stabilization, and its impact on atherosclerosis development and progression in vivo.

In conclusion, both microvascular permeability and leukocyte transmigration were increased in plaque-associated vasa vasorum, but could not be exacerbated by VE-cadherin blocking antibody treatment. This suggests that either VE-cadherin in plaque-associated vasa vasorum is already dysfunctional, and thus cannot be influenced by VE-cadherin blocking antibody treatment, or that VE-cadherin is not involved in the leaky phenotype of plaque-associated vasa vasorum. Enhancing VE-cadherin-mediated barrier function in vivo will be required to conclusively show whether there is a causal role for VE-cadherin in the leaky phenotype in plaque microvessels, and may provide a potential target for future intervention. 


\section{Supplemental Figures}
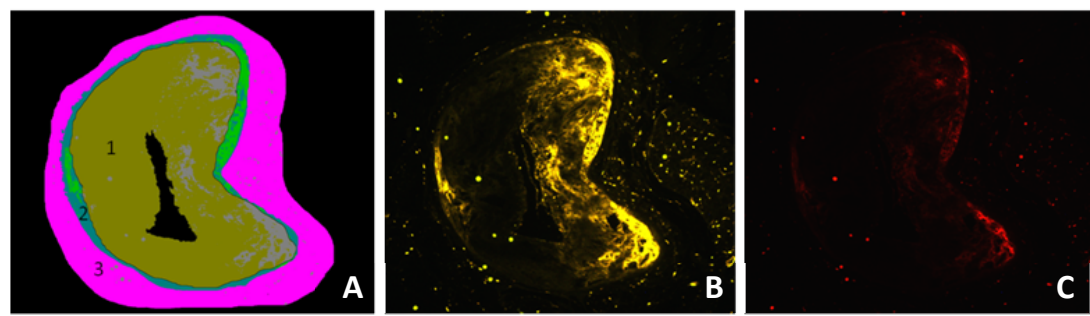

Supplemental Figure I. Quantification of the TMR positive area (A) in the intima (1), media (2) and adventitia (3) determined by autofluorescence in the OG488 channel (B) and TMR positivity in the N21 channel (C).

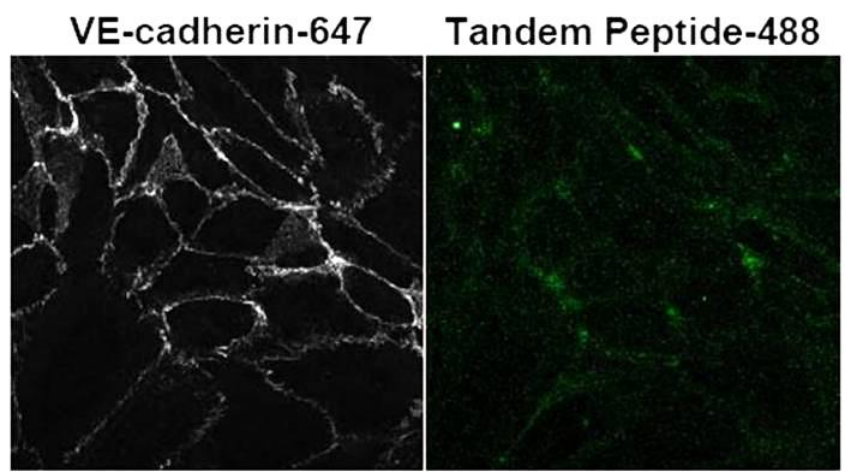

Supplemental Figure II. Untreated HUVECS incubated with VE-cadherin tandem peptide. The OG488-labeled tandem peptide was located at the endothelial junction (VE-cadherin).
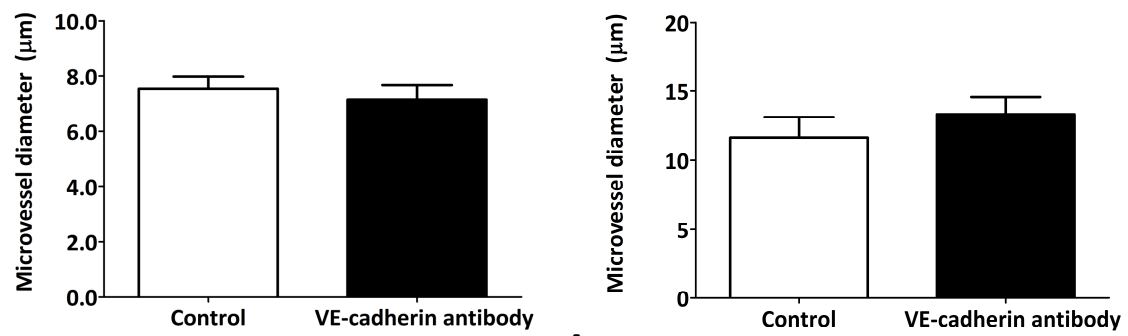

A

Supplemental Figure III. Microvessel diameter in SH microvessels (A) and plaque-associated $V V(B)$ was unaltered by treatment with VE-cadherin blocking antibody. 

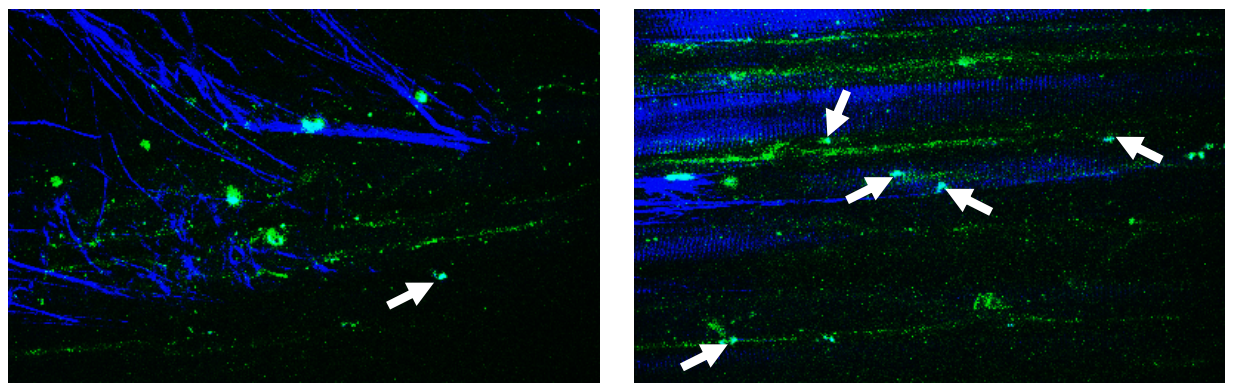

Supplemental Figure IV. Z-projection of sternohyoid muscle of old $\mathrm{LDLr}^{-/-}$mice showing collagen (blue) and microvessels (green). The number of transmigrated cells (arrows) was increased after treatment with the VE-cadherin blocking antibody (right) compared to controls (left).
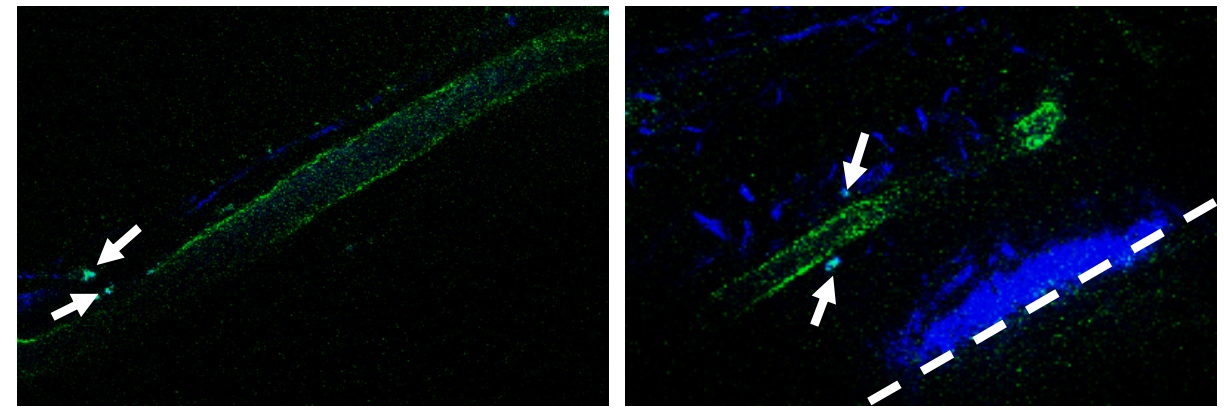

Supplemental Figure $V$. Stills from in vivo recordings from plaque-associated vasa vasorum showing of old $\mathrm{LDLr}^{-/}$mice collagen (blue) and microvessels (green). The number of adhering cells (arrows) was not increased after treatment with the VE-cadherin blocking antibody (right) compared to controls (left). Dotted line indicated outline of the carotid artery. 


\section{References}

1. Doyle B, Caplice N. Plaque neovascularization and antiangiogenic therapy for atherosclerosis. J Am Coll Cardiol. 2007;49:2073-2080

2. Rademakers T, Douma K, Hackeng TM, et al. Plaque-associated vasa vasorum in aged apolipoprotein e-deficient mice exhibit proatherogenic functional features in vivo. Arterioscler Thromb Vasc Biol. 2013;33:249-256

3. Sluimer JC, Kolodgie FD, Bijnens AP, et al. Thin-walled microvessels in human coronary atherosclerotic plaques show incomplete endothelial junctions relevance of compromised structural integrity for intraplaque microvascular leakage. J Am Coll Cardiol. 2009;53:1517-1527

4. Eriksson EE. Intravital microscopy on atherosclerosis in apolipoprotein edeficient mice establishes microvessels as major entry pathways for leukocytes to advanced lesions. Circulation. 2011;124:2129-2138

5. Garrido-Urbani S, Bradfield PF, Lee BP, et al. Vascular and epithelial junctions: A barrier for leucocyte migration. Biochemical Society transactions. 2008;36:203-211

6. Hartsock A, Nelson WJ. Adherens and tight junctions: Structure, function and connections to the actin cytoskeleton. Biochimica et biophysica acta. 2008;1778:660-669

7. Dejana E, Tournier-Lasserve E, Weinstein BM. The control of vascular integrity by endothelial cell junctions: Molecular basis and pathological implications. Developmental cell. 2009;16:209-221

8. Vestweber D. Ve-cadherin: The major endothelial adhesion molecule controlling cellular junctions and blood vessel formation. Arterioscler Thromb Vasc Biol. 2008;28:223-232

9. Vestweber D, Winderlich M, Cagna G, et al. Cell adhesion dynamics at endothelial junctions: Ve-cadherin as a major player. Trends Cell Biol. 2009;19:8-15

10. van Buul JD, van Alphen FP, Hordijk PL. The presence of alpha-catenin in the ve-cadherin complex is required for efficient transendothelial migration of leukocytes. International journal of biological sciences. 2009;5:695-705

11. Dejana E, Orsenigo F, Lampugnani MG. The role of adherens junctions and vecadherin in the control of vascular permeability. J Cell Sci. 2008;121:21152122

12. Nottebaum AF, Cagna G, Winderlich $M$, et al. Ve-ptp maintains the endothelial barrier via plakoglobin and becomes dissociated from ve-cadherin by leukocytes and by vegf. J Exp Med. 2008;205:2929-2945

13. Nawroth R, Poell G, Ranft A, et al. Ve-ptp and ve-cadherin ectodomains interact to facilitate regulation of phosphorylation and cell contacts. EMBO J. 2002;21:4885-4895

14. Broermann A, Winderlich $M$, Block $H$, et al. Dissociation of ve-ptp from vecadherin is required for leukocyte extravasation and for vegf-induced vascular permeability in vivo. J Exp Med. 2011;208:2393-2401 
15. Gotsch $\mathrm{U}$, Borges $\mathrm{E}$, Bosse $\mathrm{R}$, et al. Ve-cadherin antibody accelerates neutrophil recruitment in vivo. J Cell Sci. 1997;110 ( Pt 5):583-588

16. Orrington-Myers J, Gao X, Kouklis P, et al. Regulation of lung neutrophil recruitment by ve-cadherin. American journal of physiology. Lung cellular and molecular physiology. 2006;291:L764-771

17. Turowski P, Martinelli R, Crawford R, et al. Phosphorylation of vascular endothelial cadherin controls lymphocyte emigration. J Cell Sci. 2008;121:2937

18. Heupel WM, Efthymiadis A, Schlegel N, et al. Endothelial barrier stabilization by a cyclic tandem peptide targeting ve-cadherin transinteraction in vitro and in vivo. J Cell Sci. 2009;122:1616-1625

19. Schulte D, Kuppers V, Dartsch $\mathrm{N}$, et al. Stabilizing the ve-cadherin-catenin complex blocks leukocyte extravasation and vascular permeability. EMBO J. 2011;30:4157-4170

20. Van de Vijver P, Suylen D, Dirksen A, et al. Nepsilon-(thiaprolyl)-lysine as a handle for site-specific protein conjugation. Biopolymers. 2010;94:465-474

21. Donners MM, Wolfs IM, Olieslagers S, et al. A disintegrin and metalloprotease 10 is a novel mediator of vascular endothelial growth factor-induced endothelial cell function in angiogenesis and is associated with atherosclerosis. Arterioscler Thromb Vasc Biol. 2010;30:2188-2195

22. Schmid B, Schindelin J, Cardona A, et al. A high-level 3d visualization api for java and imagej. BMC bioinformatics. 2010;11:274

23. Zadelaar S, Kleemann R, Verschuren $\mathrm{L}$, et al. Mouse models for atherosclerosis and pharmaceutical modifiers. Arterioscler Thromb Vasc Biol. 2007;27:17061721

24. Gory-Faure S, Prandini $\mathrm{MH}$, Pointu $\mathrm{H}$, et al. Role of vascular endothelialcadherin in vascular morphogenesis. Development. 1999;126:2093-2102

25. Mitchell IC, Brown TS, Terada LS, et al. Effect of vascular cadherin knockdown on zebrafish vasculature during development. PLoS One. 2010;5:e8807

26. Bobryshev YV, Cherian SM, Inder SJ, et al. Neovascular expression of vecadherin in human atherosclerotic arteries and its relation to intimal inflammation. Cardiovasc Res. 1999;43:1003-1017

27. Hashimoto K, Kataoka N, Nakamura E, et al. Oxidized Idl specifically promotes the initiation of monocyte invasion during transendothelial migration with upregulated pecam-1 and downregulated ve-cadherin on endothelial junctions. Atherosclerosis. 2007;194:e9-17 


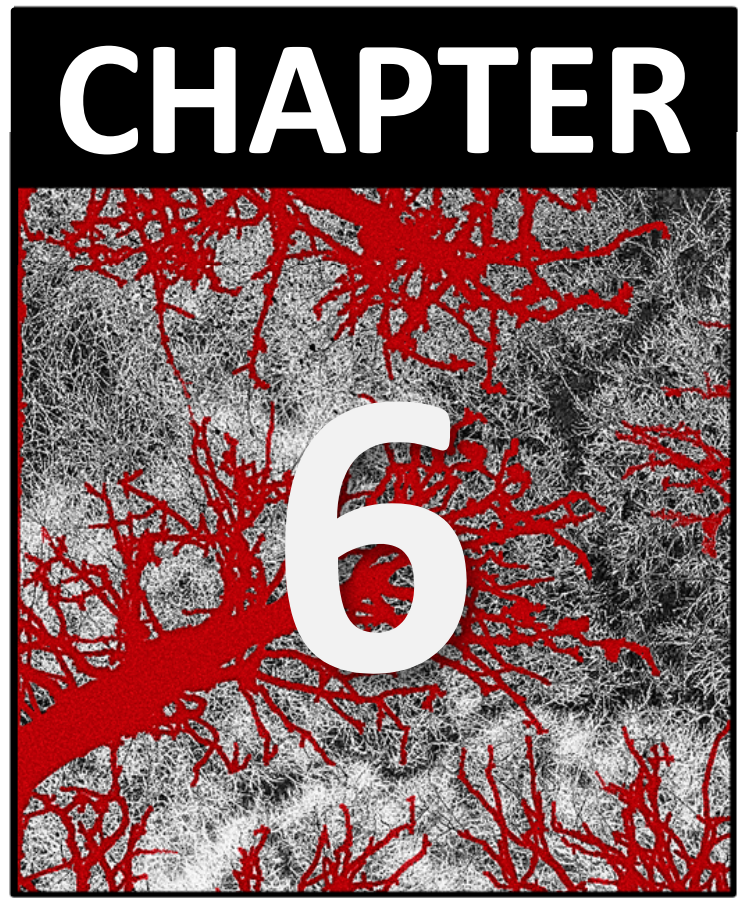




\section{Angiogenic and lymphangiogenic responses in atherosclerosis}

\section{A genomics-based approach to identify new regulators of plaque (lymph)angiogenesis}

Timo Rademakers*, Marco Manca*, Chris M. van der Loos, Allard C. van der Wal, Luc H. van den Akker, Carine J. Peutz-Kootstra, Marc A.M.J. van Zandvoort, Sylvia Heeneman, and Erik A.L. Biessen 


\section{Abstract}

During human atherosclerosis progression, plaque hypoxia and inflammation will induce a pro-angiogenic response which induces vasa vasorum expansion, the formation of intraplaque microvessels, and even lymph vessels. Although (lymph)angiogenesis has been well-characterized under physiological conditions, yet in atherosclerosis it is still poorly understood. In this study, we applied a genomics-based approach to dissect pathways and underlying regulatory cues in human plaque (lymph)angiogenesis.

Human endarterectomy samples were collected during surgery of 24 patients with plaques containing both a stable and a ruptured segment. Microvessel and lymph vessel density, relative angiogenic endothelium content, and microvessel pericyte coverage were assessed in stable and ruptured segments, showing a significant increase in microvessel density $(4.40$ \pm 0.78 vs. $7.47 \pm 1.70 \mathrm{CD} 31^{+}$vessels $\left./ \mathrm{mm}^{2} ; \mathrm{p}<0.05\right)$, lymph vessel density (3.02 \pm 1.72 vs. $16.56 \pm 2.94 \% \mathrm{D} 2-40^{+}$area; $\mathrm{p}<0.005$ ), and percentage of angiogenic endothelium in unstable plaques $(4.88 \pm 2.16$ vs. $12.00 \pm 1.84 \%$ CD105 of $\mathrm{CD} 1^{+}$vessels; $\left.\mathrm{p}<0.005\right)$. Pericyte coverage was similar in stable and ruptured segments. Microarray was performed on RNA isolated from parallel plaque tissue segments, after which expression data were correlated to histological features. Reversed engineered co-expression network analysis was performed and revealed gene modules which showed significant but essentially divergent correlations with microvessel and lymph vessel density, or percentage angiogenic endothelium. Gene modules associated with lymph vessel density and percentage angiogenic endothelium did not yield any genes for validation based on module centrality and gene significance. Gene module correlation with microvessel density exposed sixteen candidate genes for further analysis. Additionally, five miRNAs were predicted to regulate the gene module which correlated most highly with microvessel density.

In conclusion, this study identified gene and miRNA candidates which may be involved in regulating the angiogenic response in progression from advanced to ruptured plaque segments. Further in vitro and in vivo characterization and validation of these genes and miRNAs may reveal a potential role in angiogenesis and yield prospective future targets for targeted intervention. 


\section{Introduction}

The development of atherosclerotic plaques in humans has been studied extensively over the last decades. An important characteristic of developing lesions is adventitial vasa vasorum expansion, accompanied by vascularization of the intimal lesion, by ingrowth of adventitial microvessels, through the medial layer into the lesion ${ }^{1}$. Multiple human studies have shown a clear correlation between plaque stage and the extent of vascularization, as reviewed previously ${ }^{2,3}$. In addition, structural abnormalities are frequently occurring in these plaque microvessels ${ }^{4}$, potentially facilitating influx of lipids, erythrocytes, and inflammatory cells ${ }^{5}$. Beyond angiogenesis, recent studies also argue a role for lymphangiogenesis in atherosclerosis ${ }^{6,7}$, although its role is still poorly understood.

Typical angiogenic responses, both physiological and pathological, have been well studied during embryogenesis and several disease processes, most notably tumorigenesis ${ }^{8-11}$. This has led to a clear concept of the pathways involved in regulation of angiogenesis. The most well-known of these pathways is without question the Hypoxia Inducible Factor (HIF)-Vascular Endothelial Growth Factor (VEGF) axis ${ }^{12-15}$. In short, the HIF-1 and HIF-2 proteins are able to detect tissue hypoxia, leading to an upregulation of several hypoxia response genes including the VEGF-family and it receptors. VEGF in its turn will allow sprouting of new endothelial buds, and further growth of new vessels towards the hypoxic region. Beyond this classic pathway, there is a myriad of genes and proteins involved in regulating angiogenic response, be it by endothelial activation and EC junction formation (e.g. CD105, VE-cadherin ${ }^{16,17}$ ), guidance of the angiogenic sprout (e.g. DII-4, Notch, FGF, semaphorins, netrins ${ }^{15,}{ }^{18}$ ), perivascular matrix degradation (e.g. MMPs ${ }^{19}$ ), (micro)vessel stabilization (e.g. TGF- $\beta 1$, TIMPs ${ }^{20}$ ), or (micro)vessel maturation and differentiation (e.g. ephrins, angiopoietins,PDGF ${ }^{21}$ ). In recent years, several microRNAs (miRNAs or miRs) have been implicated as central regulators of angiogenesis, e.g. miR126 and miR27a/b ${ }^{22,23}$. Similar processes are involved in physiological and pathological lymphangiogenesis, which shows great homology to angiogenesis, and often involves family members of molecules involved in the angiogenic process, e.g. VEGFs, VEGFRs, and neuropilins (NRP) ${ }^{24}$. 


\section{CHAPTER 6}

Many of the angiogenic factors mentioned have been studied in atherosclerosis ${ }^{1,2,10,25,26}$. However, the majority are not viable as targets for (clinical) intervention, in view of adverse systemic side-effects $1,11,27$. Obviously, genes specifically involved in the pathologic angiogenic process during atherosclerosis development and progression would represent a much more suitable clinical target.

A genomics approach would be very suitable for identifying such novel, atherosclerosis specific targets. By correlating gene expression patterns to (lymph)angiogenesis certain traits, gene networks can be built revealing central hubs that are functionally related to these processes. Related approaches have already been employed in for instance oncology to discover new markers for tumor-derived endothelium ${ }^{28}$, to detect expression signatures of miRNAs related to angiogenesis in tumors ${ }^{29}$, to find biomarkers for endothelial cell proliferation in tumors ${ }^{30}$, or to define response of tumor therapy by investigating expression profiles ${ }^{31}$.

Here, we used a method in which we combined data on plaque angiogenesis based on histology and immunohistochemistry with a microarray analysis on the same tissue. By this approach, we not only were able to demonstrate that angiogenesis, lymphangiogenesis and vessel maturation are associated with distinct regulatory gene modules, but also set forth to identify new genetic targets involved in the regulation of plaque angiogenesis and lymphangiogenesis in human atherosclerosis. 


\section{Methods}

\section{Patient samples, histology and immunohistochemistry}

Human atherosclerotic plaque samples were obtained from carotid artery lesions from 24 patients undergoing endarterectomy (Department of Surgery, Orbis Medical Center, Sittard-Geleen, the Netherlands). The tissue was obtained from the Maastricht Pathology Tissue Collection (MPTC), and collection, storage, and use of tissue and patient data were performed in agreement with the Dutch Code for Proper Secondary Use of Human Tissue. Immediately after resection, the atherosclerotic tissue was divided into parallel segments of $5 \mathrm{~mm}$. Snap frozen segments for RNA isolation were alternated by formalin-fixed segments for histology and were only included when both adjacent haematoxylin-eosin (HE) stained sections were classified as advanced stable or ruptured atherosclerotic lesions. Plaques were staged by histological analysis based on HE staining of adjacent slides according to Virmani et al, where thick fibrous cap atheroma (TkFCA) were classified as advanced stable, and intraplaque hemorrhage (IPH) as ruptured segments, respectively ${ }^{32}$. Patients were selected for having an advanced stable and ruptured snapfrozen segment within the lesion (advanced stable $(n=21)$ and ruptured $(n=23)$ segments).

Immunohistochemical (IHC) stainings were performed on consecutive paraffin sections for vascular endothelial marker CD31 (Dako), the angiogenic marker CD105 (Thermo Scientific), the smooth muscle cell/pericyte aSMA (Dako), the macrophage marker CD68 (Dako), the lymphatic endothelial marker D2-40 (Dako), and the lymphatic transcription factor Prox-1 (Prof. Dr. Alitalo, University of Helsinki, Finland).

In all sections, the total amount of $\mathrm{CD} 31^{+}$microvessels, D2-40 lymph vessels and $\mathrm{CD} 8^{+}$macrophages in the plaque was counted by two observers and corrected for plaque size, yielding the microvessel density (MVD; vessels $/ \mathrm{mm}^{2}$ ), lymph vessel density (LVD; \%D2-40 vessel area of plaque area), or lesional macrophage content (\% of total plaque area).

Double staining for CD31/CD105 to assess the percentage of angiogenic endothelium, was analyzed with the Nuance spectral imaging system (Caliper Life Science). Multispectral imaging (MSI) data sets were taken from $420-720 \mathrm{~nm}$ at $20 \mathrm{~nm}$ intervals using a DM-5000 Leica microscope system at 20x (Plan Apo). Spectral libraries of single-red (Vector Red), single-blue 
(Vector Blue), single-brown (DAB), and natural occurring iron pigments were obtained from the control slides. The resulting library was applied to the double stained slides to spectrally dissect/unmix them into individual component images using the Nuance ${ }^{\mathrm{TM}} 3.0$ software ${ }^{33}$. Pseudo-color images showing co-localization, as well as an exclusive image of co-localization, were generated with the Nuance 3.0 software. Quantitative assessment of colocalization and pixel-based measurement of the individual markers per microscopic field was done with the same software.

In addition, the amount of $\alpha$ SMA covered microvessels and the aSMAcoverage per microvessel was quantified morphometrically using Leica QWin V3.0 (Leica).

\section{Statistical analysis of patient characteristics and immunohistochemistry}

All data on patient characteristics and immunohistochemistry are presented as mean \pm SEM. Statistics were performed using Graphpad Prism 5.0. Groups were compared using a Mann-Whitney rank-sum test for continuous variables, and were considered statistically different when $p<0.05$.

\section{RNA extraction \& transcriptomics}

RNA isolation was performed by Guanidium Thiocyanate lysis followed by Cesium Chloride gradient centrifugation. Following extraction, the RNA was further purified using the Nucleospin RNAll kit (Macherey-Nagel GmbH \& Co. KG). RNA concentration was measured using a Nanodrop ND-1000 spectrophotometer (Nanodrop Technologies). The RNA quality and integrity was determined using Lab-on-Chip analysis on an Agilent 2100 Bioanalyzer (Agilent Technologies). The lowest RNA Integrity Number (RIN) was 5.6, the average RIN being $7.23 \pm 0.48$.

Biotinylated cRNA was prepared using the Illumina TotalPrep RNA Amplification Kit (Ambion, Inc.) according to the manufacturer's specifications starting with 100 ng total RNA. 750 ng of cRNA per sample was used for hybridization. Hybridization to Illumina Human Sentrix-8 V2.0 BeadChip ${ }^{\circledR}$ and washing were performed according to the Illumina standard assay procedure. Scanning was performed on the Illumina BeadStation 500 (Illumina Inc.), image analysis and extraction of raw expression data was performed with Illumina Beadstudio v3 Gene Expression software with default settings (no background subtraction) and no normalization. 


\section{Computational methods}

Analyses have been performed in the software module $\mathrm{R}^{34}$. Raw expression data were imported using the package lumi ${ }^{35}$, and its Variance Stabilizing Transform was used for background correction. Data were normalized by robust spline normalization, and a filter was imposed to exclude all those probes whose detection call resulted ambiguous (30 or more readings of one probe with a p-value higher than 0.01) (Supplemental Figure I).

Expression of CD31, CD105, D2-40 and aSMA in the microarray data was consequently correlated to histological data. Reverse engineering of a coexpression network was performed by taking advantage of the tools included in the package WGCNA ${ }^{36}$. Softhreshold Power was set at 10 . Modules, clusters of densely interconnected genes, were identified using the default method of hierarchical clustering ${ }^{37}$ and the function 'cutreeDynamic' ${ }^{38}$, with 'minModuleSize $=30$ ' and 'deepSplit=2', to "cut" the branches of the tree. The function 'moduleEigengenes' was used to calculate modules Eigengene, and the function 'mergeCloseModules' to optimize the heuristics of modules by merging those who's Eigengenes were highly correlated.

For distinguishing between stable and ruptured sections of the same plaque, consensus modules, preserved across the two conditions, were identified by the function 'blockwiseConsensusModule'. We assumed that consensus gene clusters would be reflective of biological processes underlying plaque progression towards instability. By contrast, modules only identified in ruptured samples would be reflective of the changes induced by rupture.

Module-traits association was quantified by correlation and by univariate regression model of the Eigengene and quantitative trait, with a $p$ value threshold $\leq 0.05$. Within each module, genes with high module membership in modules related to traits were natural candidates for further investigation, as suggested by Langfelder and Horvath ${ }^{39,}{ }^{40}$. Hereafter, we exported the network data to Cytoscape for visualization and further analyses ${ }^{41}$. The plugin ClueGO was used to enrich the modules of interest for over-represented Gene Ontology terms and Pathways ${ }^{42}$. 


\section{Results}

Microvessel density was increased in ruptured segments and was associated with an increase in CD105, whereas $\alpha$ SMA coverage was unaltered

To assess plaque and lipid core size, paraffin-embedded sections were $\mathrm{HE}$ stained and revealed an increased plaque (Figure $1 \mathrm{~A}$ ) and lipid core size (Figure $1 B$ ) in ruptured segments compared to stable segments. Staining for the vessel marker CD31 showed both increased MVD (Figure 1C) and microvessel hotspot numbers (Figure 1D) within the plaque. Co-localization of the angiogenic marker CD105 and CD31 was assessed in stable and ruptured segments and analyzed using multispectral analysis (Figure 1E), showing an augmented percentage of angiogenic plaque microvessels (Figure 1F). Pericyte

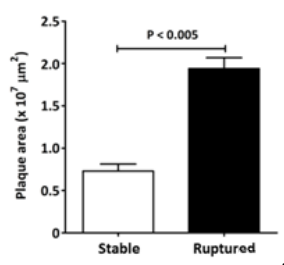

A
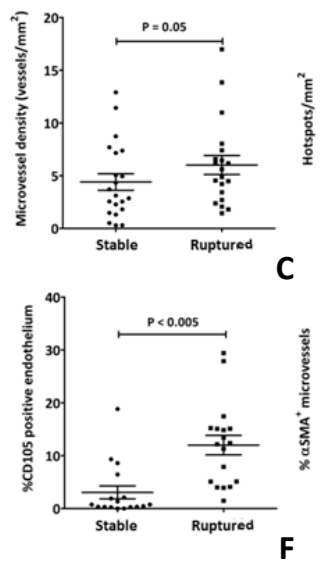

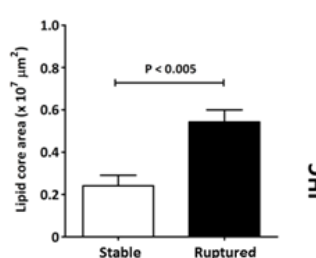

B
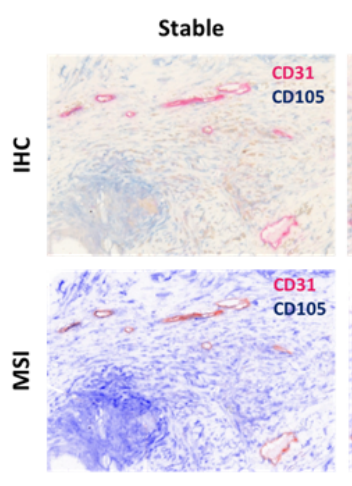

D
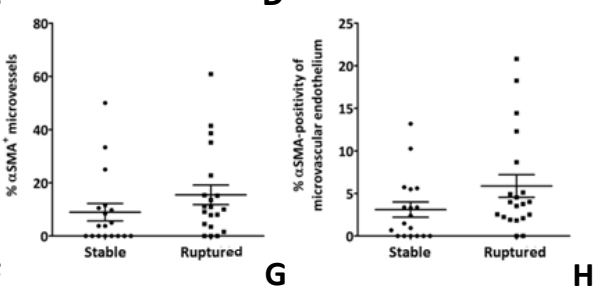

Ruptured

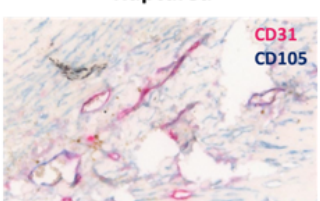

C.31:

CD105.

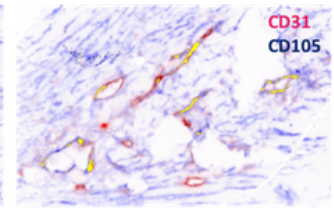

E

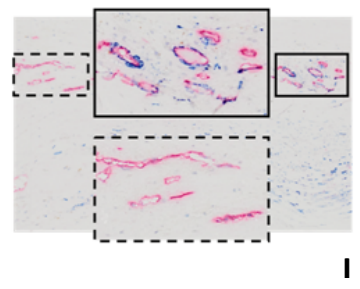

Figure 1. Ruptured segments showed an increase in microvessel density, angiogenically active endothelium, but no difference in pericyte coverage. Plaque area (A) and lipid core area (B) were quantified based on HE staining and were increased in ruptured segments. Concomitantly, MVD (C) and the number of intimal microvascular hot spots $(D)$ were increased in ruptured segments. The percentage of $\mathrm{CD} 105^{+}$angiogenic endothelium was quantified by colocalization analysis (yellow) using multispectral imaging (E), and showed a significant increase in ruptured segments (F). The pericyte coverage (G), as well as the aSMA-positive area per microvessel (H) was unchanged, although heterogeneity in aSMA-coverage (blue) between different $C D 31^{+}$hotspots (red) was clearly visible (I). 
coverage defined as aSMA-covered microvessels (Figure 1G) and the amount of aSMA-positivity per microvessel (Figure $1 \mathrm{H}$ ) were measured, and did not show differences between stable and ruptured segments, although a high heterogeneity could be observed within the segments.

\section{Extensive lymph vessel expansion was present in rupture segments}

Besides the expansion of plaque microvessels in human plaques, recent data suggested proliferation of $\mathrm{D} 2-40^{+}$lymph vessels in advanced stages of atherosclerosis ${ }^{7}$. Accordingly, we performed D2-40 staining, targeting the specific lymphatic endothelial cell marker podoplanin, which revealed strong presence of D2-40 in our set of atherosclerotic lesions (Figure 2A), especially in the shoulder regions (Figure 2A, inset). When compared to stable segments, LVD was increased 5 -fold in ruptured segments (Figure 2B). To confirm that the D2- $40^{+}$vessels indeed exclusively represented lymph vessels, a double staining with the lymphatic transcription factor Prox-1 was performed. D2-40/Prox-1 double positive lymph vessels were specifically shown in control lymphoid tissue (Figure $2 \mathrm{C}$ ) and within atherosclerotic lesions (Figure 2D), affirming that the vessel shaped D2-40 $0^{+}$structures were in fact lymph vessels.
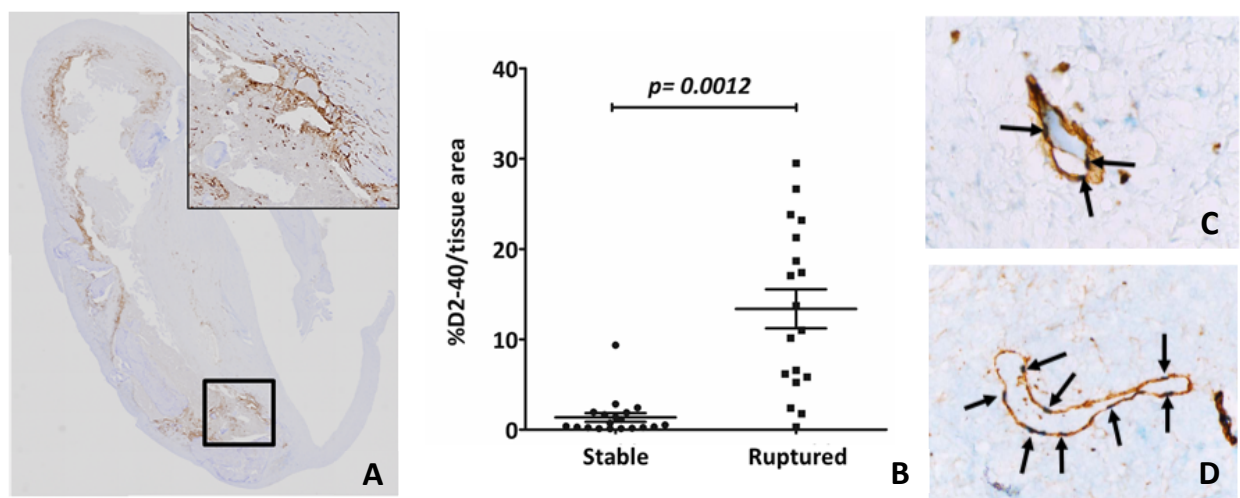

Figure 2. Expression of the lymphatic marker D2-40 in stable and ruptured segments. $D 2-40^{+}$ lymph vessels (A, brown) are abundantly present in ruptured segments in comparison to stable segments (B). Specificity of D2-40 (brown) was determined by double staining with the lymphatic transcription factor Prox-1 (blue) in control tonsil tissue (C) and in atherosclerotic lesions (D). 
Plaque vascularization and lymph vascularization were regulated by independent gene clusters

Quantified histological phenotypes were correlated to microarray data, allowing normalization for the histological traits. Correlation analysis of histologic traits was performed, to exclude co-linearity between traits which would influence later module-phenotype correlations, and showed no colinearity, except for the expected co-linearity between MVD and microvessels numbers (not shown). In addition, patient samples were clustered hierarchically and a heatmap of histological traits was constructed to assess possible segregation of patients samples based on histological traits (Supplemental Figure II). None of the patients showed significant segregation from the study population.

Subsequently, a weighted co-expression network was constructed by implementing a gene co-expression similarity measure to relate every pairwise gene-gene relationship. To identify gene clusters, the network was partitioned into different modules whenever the number of connections of gene cluster to their immediate surroundings was higher than the number of connections shared with the center of the group (Supplemental Figure III). This approach resulted in the formation of network modules, consisting of groups of densely interconnected genes. Eventually, 38 gene modules could be distinguished and were subsequently correlated to the histological traits and visualized in a module-trait heatmap, to identify modules which are most significantly associated with a certain trait like MVD, LVD or angiogenic activity of lesional microvascular endothelium (Figure 3 ). MVD was highly correlated with module $L\left(p=1.0 \times 10^{-4}\right)$, LVD with module $W\left(p=1.0 \times 10^{-4}\right)$, and angiogenic activity with module $R(p=0.004)$. Interestingly, MVD and lesion macrophage content showed a great, yet not significant overlap in upregulated modules. The modules which correlated most highly with specific phenotypic traits were investigated in more detail. 


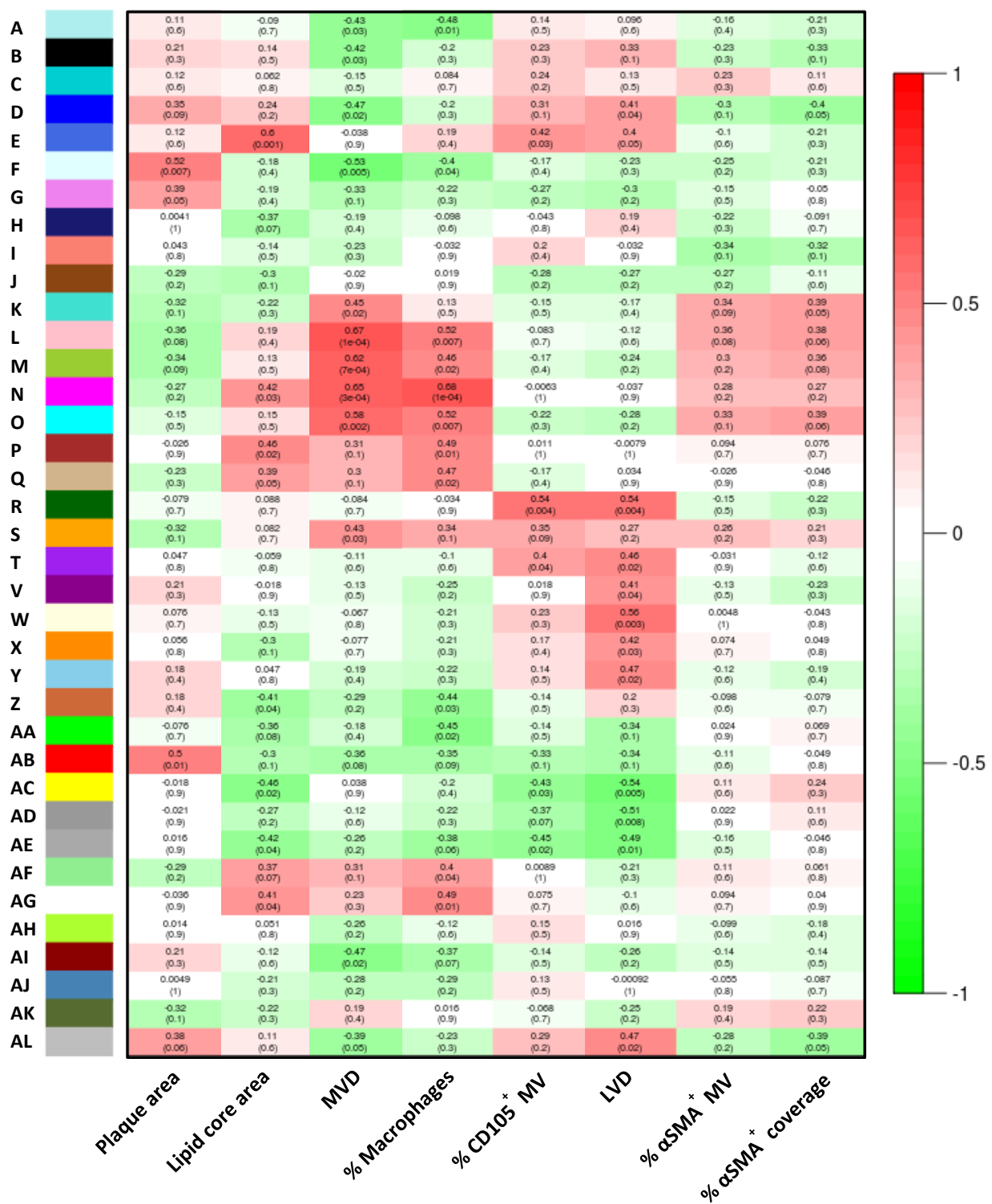

Figure 3. Heatmap of the module-trait correlations, showing upregulated (red) or downregulated (green) gene modules in relation to phenotypic traits in ruptured segments. $A$ high overlap in genetic profile was found for MVD and lesional macrophage content (\% Macrophages), while the percentage angiogenic endothelium (\% $\mathrm{CD}_{105^{+}} \mathrm{MV}$ ) showed a completely different expression profile. LVD showed some overlap with gene clusters associated with angiogenic endothelium, but was separated from e.g. MVD. Module W was especially enriched for LVD. $\alpha S M A$ parameters showed certain overlap with MVD, but failed to show highly enriched modules. Values of the Pearson's $r$ coefficient and associated $p$-values (in parenthesis) are reported. 


\section{CHAPTER 6}

Analysis of the gene cluster highly correlating with MVD, but not with angiogenic activity, yielded potential target genes involved in plaque neovascularization

To identify potential central genes involved in plaque neovascularization, the genes from module $\mathrm{L}$ were assessed with respect to the correlation between their centrality within module $L$ and gene significance for MVD (Figure 4). By this approach, genes which are most likely to be functionally involved in determining MVD, in other words plaque neovascularization, displayed highest correlation to both centrality and gene significance. As a cutoff for further gene selection, we applied a minimum value of 0.7 for centrality and gene significance to the module (Figure 4), reducing the number of genes for functional testing in vitro to 16 (Table 1).

Identical analyses were performed for module $\mathrm{R}$ which was highly correlated to angiogenic activity of the microvascular endothelium in the lesion $\left(p=1.7 \times 10^{-4}\right.$ ). Module correlation was however low (25\%) and none of the genes in this module met the criteria (cut-off values) proposed above (Supplemental Figure IV).

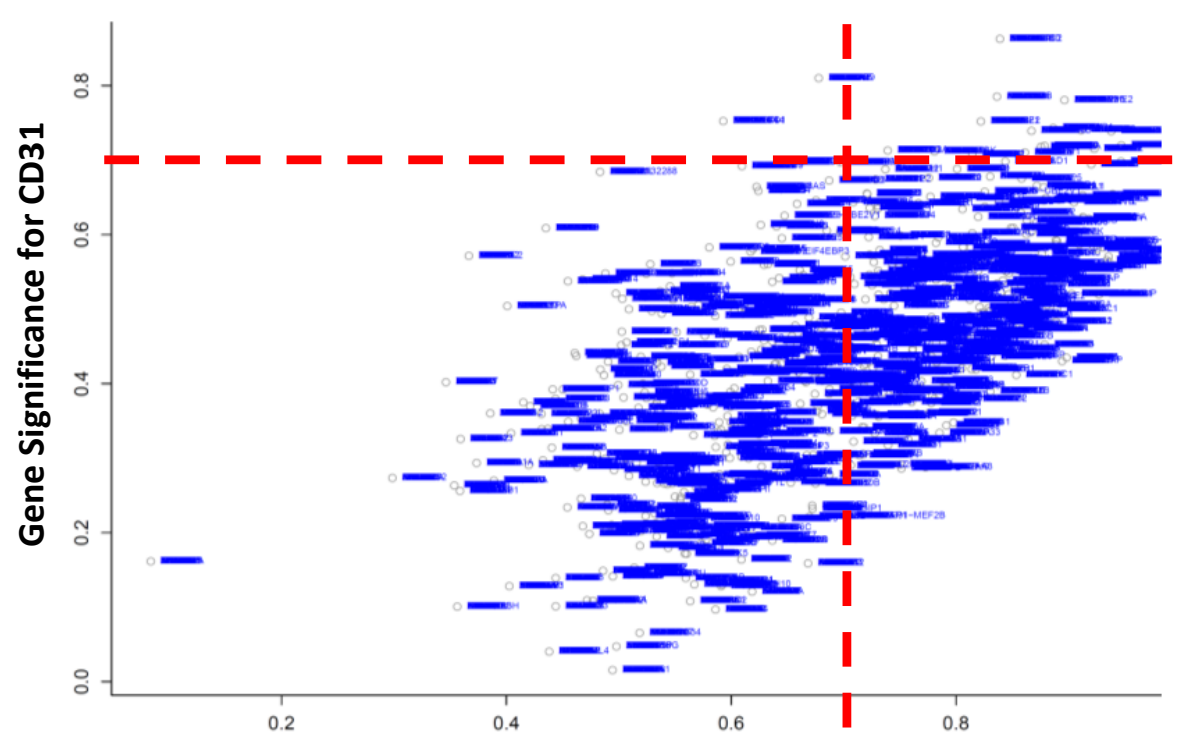

Module membership for Module L

Figure 4. Correlation of gene module centrality versus significance of association to the phenotypic parameter MVD. Genes which are most likely to be functionally involved in determining MVD can be detected by exhibiting a high correlation to both centrality and gene significance. Cut-off value was 0.7 for both parameters (dotted lines). 
Table 1. Genes showing high correlation for centrality within module L and great gene significance for CD31

\begin{tabular}{ll|cccc}
$\begin{array}{l}\text { Gene } \\
\text { symbol }\end{array}$ & $\begin{array}{l}\text { Entrez } \\
\text { ID }\end{array}$ & $\begin{array}{c}\text { Significance } \\
\text { for MVD }\end{array}$ & $\begin{array}{c}\text { P-value Gene } \\
\text { Significance }\end{array}$ & $\begin{array}{c}\text { Centrality in } \\
\text { Module L }\end{array}$ & $\begin{array}{c}\text { P-value Module } \\
\text { Centrality }\end{array}$ \\
\hline ABCA9 & 10350 & 0.86 & $2.86 \mathrm{e}-08$ & 0.84 & $1.61 \mathrm{e}-07$ \\
CNTNAP3 & 79937 & 0.81 & $9.16 \mathrm{e}-07$ & 0.68 & $1.96 \mathrm{e}-04$ \\
CASK & 8573 & 0.79 & $3.32 \mathrm{e}-06$ & 0.84 & $1.91 \mathrm{e}-07$ \\
ZEB1 & 6935 & 0.78 & $4.13 \mathrm{e}-06$ & 0.90 & $1.34 \mathrm{e}-09$ \\
PFN2 & 5217 & 0.75 & $1.44 \mathrm{e}-05$ & 0.82 & $4.64 \mathrm{e}-07$ \\
PPP2CB & 5516 & 0.74 & $2.06 \mathrm{e}-05$ & 0.89 & $3.75 \mathrm{e}-09$ \\
RPL32 & 6161 & 0.74 & $2.29 \mathrm{e}-05$ & 0.91 & $2.45 \mathrm{e}-10$ \\
ACSL3 & 2181 & 0.74 & $2.40 \mathrm{e}-05$ & 0.87 & $2.06 \mathrm{e}-08$ \\
CAV2 & 858 & 0.74 & $2.43 \mathrm{e}-05$ & 0.94 & $4.54 \mathrm{e}-12$ \\
TMEM98 & 26022 & 0.72 & $4.95 \mathrm{e}-05$ & 0.95 & $7.82 \mathrm{e}-13$ \\
RNF11 & 26994 & 0.72 & $5.10 \mathrm{e}-05$ & 0.88 & $7.50 \mathrm{e}-09$ \\
ECM2 & 1842 & 0.72 & $5.33 \mathrm{e}-05$ & 0.88 & $1.01 \mathrm{e}-08$ \\
PPAP2A & 8611 & 0.72 & $5.86 \mathrm{e}-05$ & 0.92 & $5.26 \mathrm{e}-11$ \\
ARMCX6 & 54470 & 0.71 & $6.54 \mathrm{e}-05$ & 0.78 & $4.11 \mathrm{e}-06$ \\
TGFB2 & 7042 & 0.71 & $6.93 \mathrm{e}-05$ & 0.86 & $3.27 \mathrm{e}-08$ \\
CNPY4 & 245812 & 0.71 & $7.62 \mathrm{e}-05$ & 0.81 & $7.23 \mathrm{e}-07$
\end{tabular}

Analysis of gene cluster highly correlating with LVD, did not reveal genes potentially relevant for plaque lymphangiogenesis

Similar to the approach we applied for the genes from module $L$, we assessed the correlation between the centrality within module $W$ and gene significance for LVD for genes of module W (Supplemental Figure V). Unexpectedly, this module was only comprised of a relatively small number of genes, and none of the genes met the criteria for centrality and gene significance. Also two additional modules with high correlation to LVD, modules T and AL, showed identical results. 
Reverse network engineering predicted putative miRNAs involved in gene regulation within the module associated with MVD

In addition to the previous analyses, the connectivity matrix representing module $L$ was transferred in Cytoscape, where the network architecture was investigated and an enrichment analysis for regulatory mechanisms was performed on the basis of the gene-gene interactions. Interestingly, this approach revealed five miRNAs (miR302d, miR488*, miR582-3, miR606 and miR1284) which were predicted to be centrally present within the gene network, and which could potentially be of importance for regulation of the genes linked to MVD (Figure 5).

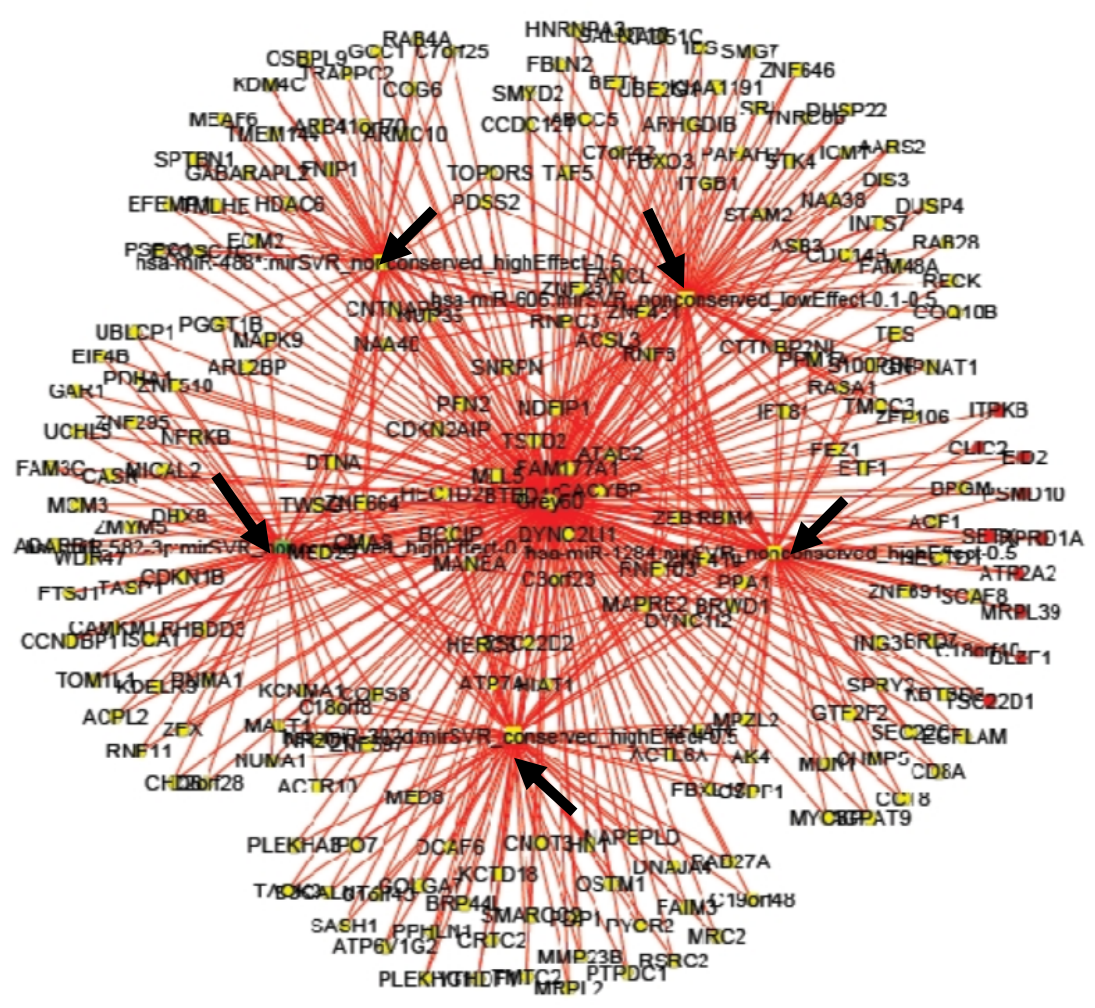

Figure 5. Gene clustering of module L. Network analysis predicted five miRNAs (miR302d, miR488*, miR582-3, miR606 and miR1284) to be centrally present (arrows) within the gene network, potentially involved in the regulation of genes linked to MVD. 


\section{Discussion}

In this study, we identified genes, transcription factors, and miRNAs which are specifically regulated in plaque angiogenesis and lymphangiogenesis, and may thus be indirectly involved in plaque rupture and intraplaque hemorrhage. We correlated quantified histological data with expression data from a microarray performed on stable and ruptured atherosclerotic tissue segments, and attempted to dissect regulatory pathways that control or are affected by plaque (lymph)angiogenesis. We were able to distil a number of genes which may be involved in the (lymph)angiogenic process in atherosclerosis, as well as miRNAs which thus far are unknown both in atherosclerosis and angiogenesis.

In the current study, we first assessed phenotypical traits based on histology and immunohistochemistry. Histological analysis showed an increase in plaque and lipid core size in ruptured segments. Concurrently, and in line with literature ${ }^{3,7}$, we found an increased microvascular density, lymph vessel density, and increased angiogenic activity in ruptured segments compared to stable segments. As expected, pericyte coverage was low, yet surprisingly not differential between the stable and ruptured segments, and showed a highly heterogeneous pattern of pericyte coverage. Low pericyte coverage has been described in tumor vasculature ${ }^{43}$, but was until now not extensively investigated in plaque neovasculature. An important conclusion is that absence of pericytes is a common feature in advanced atherosclerotic lesions, and that an immature microvascular phenotype is already present prior to progression towards a ruptured or vulnerable plaque stage. This also suggests that newly formed microvessels in plaques do not undergo vessel maturation, and that the low pericyte coverage is not due to pericyte loss during plaque progression. To verify this however, pericyte coverage should be thoroughly quantified in several, also earlier, plaque development stages.

From the histological analysis, data was correlated with expression data from a microarray. The large amount of genes found in the CD31 correlation analysis required us to include further criteria for gene selection for validation. Implementing a strict cut-off value for correlation with the gene of interest with the module (Gene Significance) and a central position within the module (Module Membership) resulted in a great reduction in the number of genes. Although the cut-off value is in part arbitrary, we consider a high 
correlation with both factors important, since this would reflect genes which likely have a central role in regulation of plaque neovascularization in vivo.

Several of the genes we identified within module $L$ (Table 1 ) could already represent interesting targets based on their previously described functions. Examples hereof are PFN2, CNTNAP3, or CASK, which have been linked to neuronal growth, neuronal guidance, or response to hypoxia ${ }^{44-46}$. Specifically for finding new potential angiogenesis-related genes, genes which have already been described in neuronal growth could be of special interest, since it has been shown that there is a great homology between genes involved in neuronal growth and angiogenesis ${ }^{47}$. Therefore, it is conceivable that genes described in this process may also be involved in plaque angiogenesis. Additionally, several known angiogenesis-related genes, e.g. FGF-2, were expressed in module L, although their module centrality and gene significance were below the cut-off values applied for gene selection.

Interestingly, the modules correlating with analysis of the gene cluster which highly correlated with angiogenic endothelium did not reveal any relevant targets. This was unexpected, as one may expect that selection based on a marker which is regarded as a marker of angiogenesis yields specific angiogenic targets. One explanation may be that CD105, although it is known to be expressed on activated endothelium, can also be found on endothelial cells, activated macrophages, fibroblasts, and smooth muscle cells ${ }^{48}$. Even though we specifically analyzed CD105 expression on CD31 ${ }^{+}$ endothelium, whole tissue expression of CD105 in the atherosclerotic tissue may have obscured its function in angiogenesis in the microarray.

Not only correlation with CD105 expression yielded poor results. Also the amount of genes correlating with the lymphatic marker D2-40 was relatively low. Module $\mathrm{W}$, most highly correlating with LVD, only consisted of few genes, none of which met the criteria (cut-off values) set for inclusion for further validation. Including alternative markers for lymphangiogenesis for correlation to the microarray data, or defining slightly less stringent cut-off values for gene inclusion may be options for future analysis.

The module correlating to CD31 expression is also strongly correlated to the expression of the macrophage marker CD68. It is known that macrophages can produce high levels of angiogenic and potentially lymphangiogenic factors, especially under inflammatory conditions. In addition, macrophages produce a multitude of factors required for the 
angiogenic process, e.g. proteases or guidance molecules ${ }^{49,50}$. Therefore, the fact that the same module is strongly correlating with both endothelial cells and macrophages is not surprising, and may reflect the interplay between microvessels and macrophages in plaque angiogenesis. Some of the genes described in Table 1 may also reflect the contribution of macrophages, as e.g. ABCA9 has been previously linked to lipid uptake by macrophages ${ }^{51}$. The overlap in gene expression profile, however, substantiates the need for proper gene selection for validation, as mentioned earlier e.g. by looking at cell type specific expression. These studies are currently underway.

Of great interest is our finding of novel miRNAs which may be involved in plaque angiogenesis. These miRNAs, non-coding, single-stranded RNAs which are important regulators of gene expression, have received a lot of attention in the last few years. Consequently, several miRNAs have been implicated in angiogenesis, or in related processes like migration, as recently reviewed by Chen et $a l^{52}$. The miRNAs we found have thus far not been linked to angiogenesis, and except for miRNA606, which was detected in a miRNA screening in colorectal cancer ${ }^{53}$, no function has been attributed to these miRNAs. This makes these miRNAs valuable targets for further validation.

An imperative next step definitively will be validation of these genes and miRNAs, both in vitro and in vivo. A first step in this would be to characterize expression of these genes and miRNAs in endothelial cells (and macrophages). Additionally, the genes and miRNAs may be further studied functionally by inhibiting gene or miRNA expression. For endothelial cells, a number of functional parameters could be studied, e.g. adhesion, proliferation and migration capacity, the ability of the endothelial cells of form vessels in a tube formation assay, or the assessment of endothelial barrier function, investigating a potential role in the leaky phenotype of vessels. Viable candidates, which show a disturbed function after knock-out, may additionally be tested in vivo, e.g. in a zebrafish system ${ }^{54,55}$. This will allow validating in vitro data and would be a high-throughput system for selecting the most promising candidate for testing in a murine atherosclerosis setting.

In conclusion, we present several genes and five miRNAs which may have a role in control of, or are affected by plaque (lymph)angiogenesis. Validation of the genes and miRNAs will be necessary to determine whether these genes are indeed involved in these processes and may potentially yield future targets for specific intervention. 


\section{Supplemental Figures}
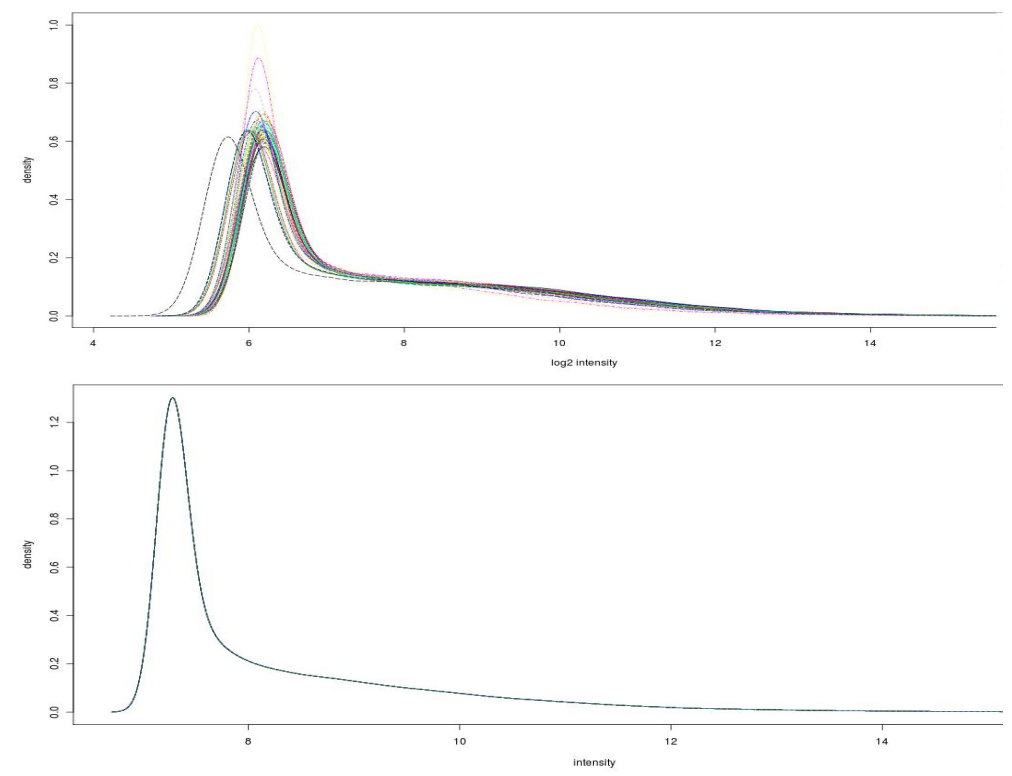

Supplemental Figure I. Density histogram of raw log-intensities from patient samples before (upper panel) or after (lower panel) preprocessing. The overlap of samples after normalization confirms that batch effect has been eradicated and that all the signals share the same scale.

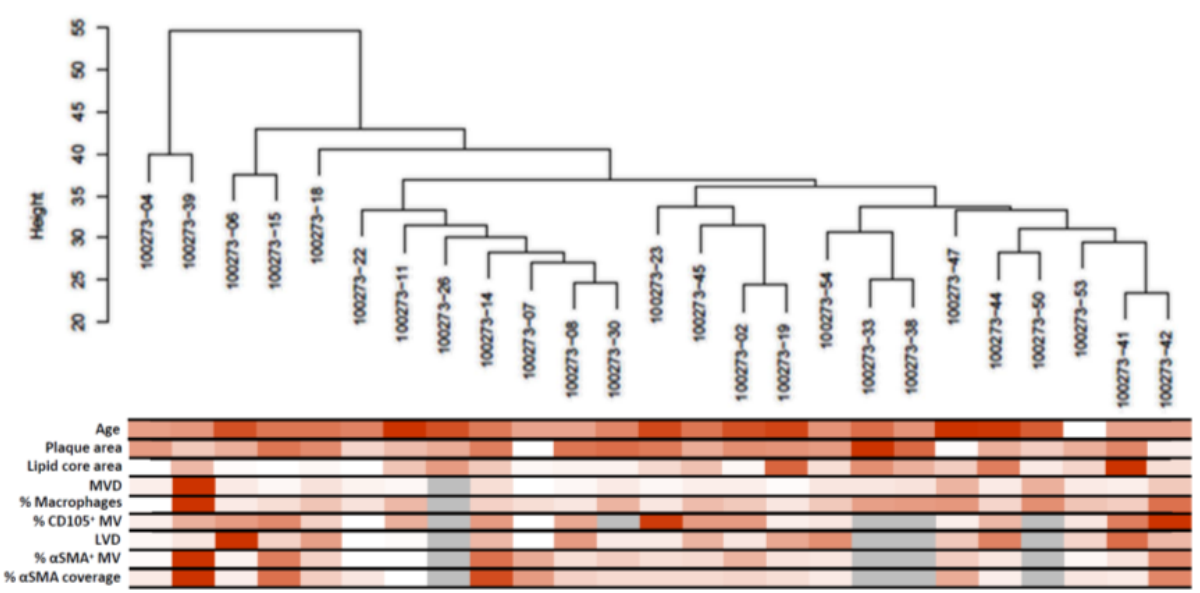

Supplemental Figure II. Sample dendrogram and trait heatmap, showing homogeneous distribution of patients, with no outliers, and revealing the homogeneity of the impact of each included sample on the value distributions of the quantified phenotypes 


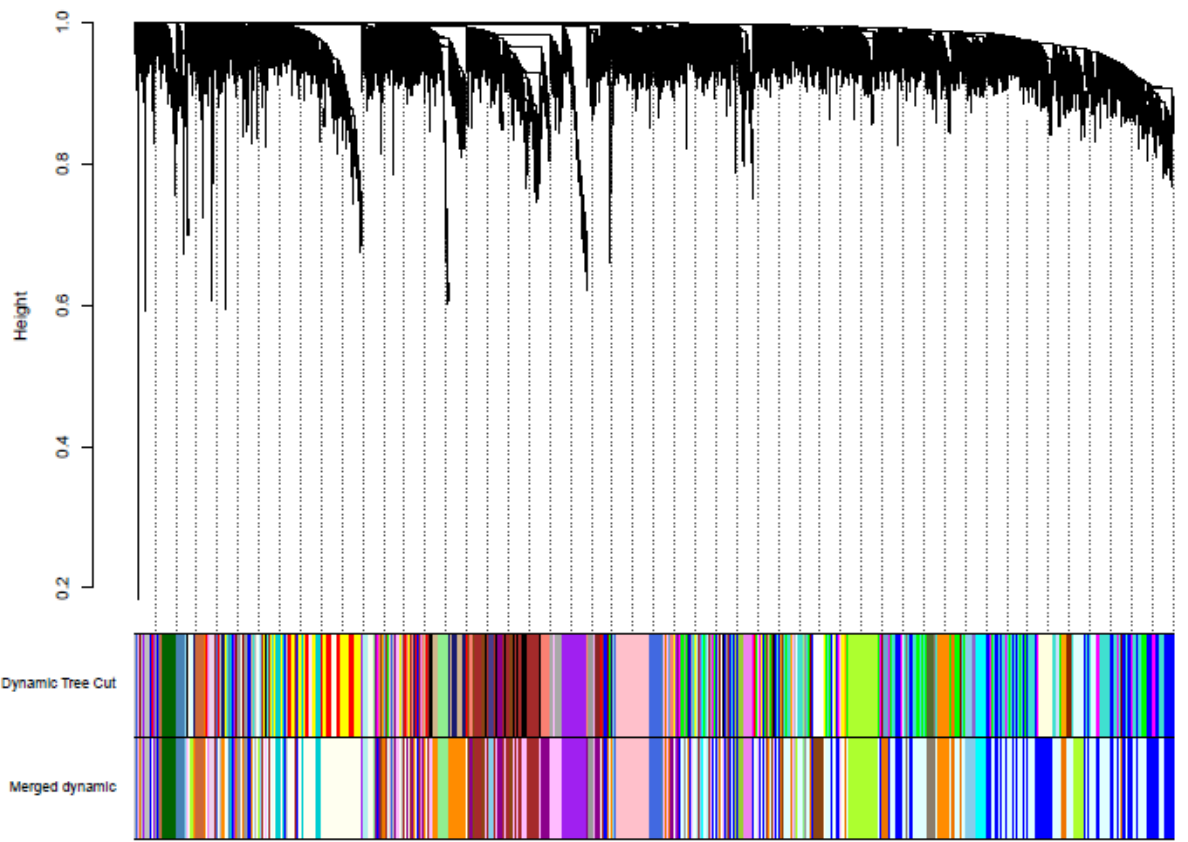

Supplemental Figure III. Cluster dendrogram showing clustering of gene networks into modules. Whenever the number of connections of gene cluster to their immediate surroundings was higher than the number of connections shared with the center of the group, this network was classified as a module.

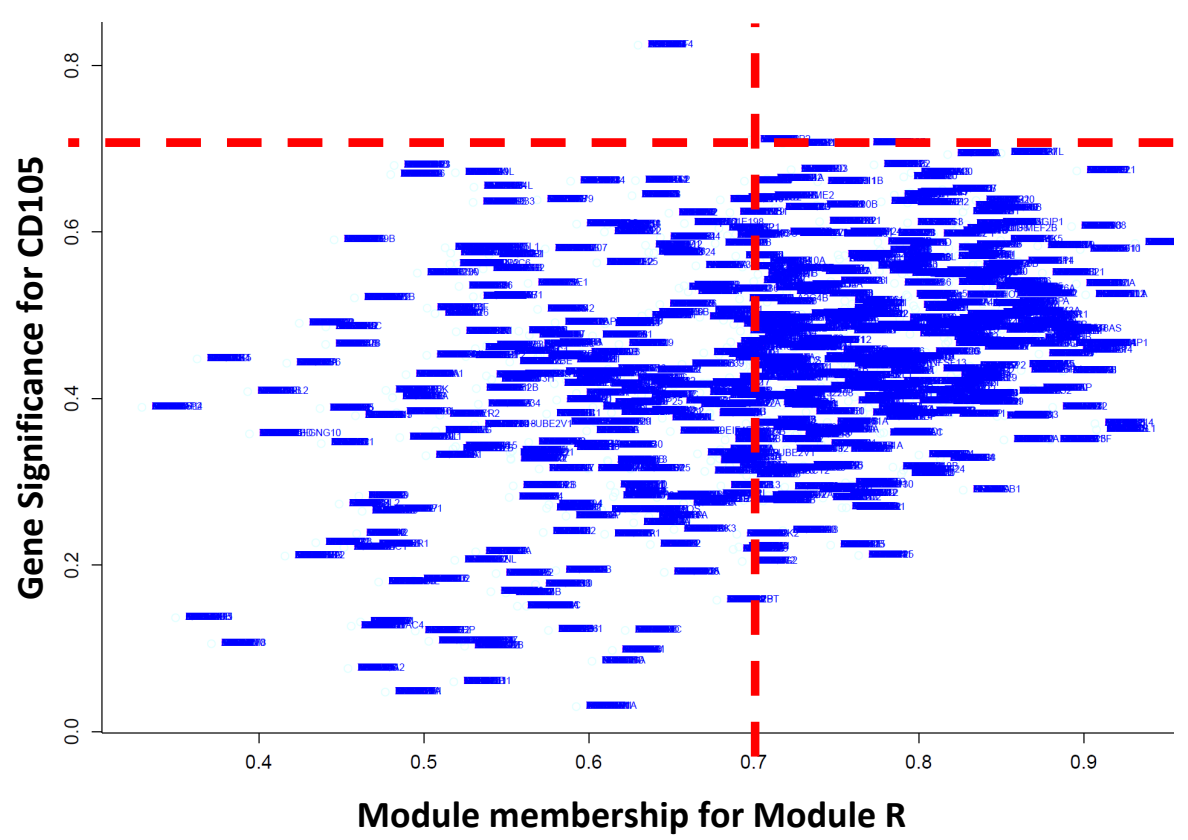

Supplemental Figure IV. Module Membership for Module R versus Gene Significance for CD105. 


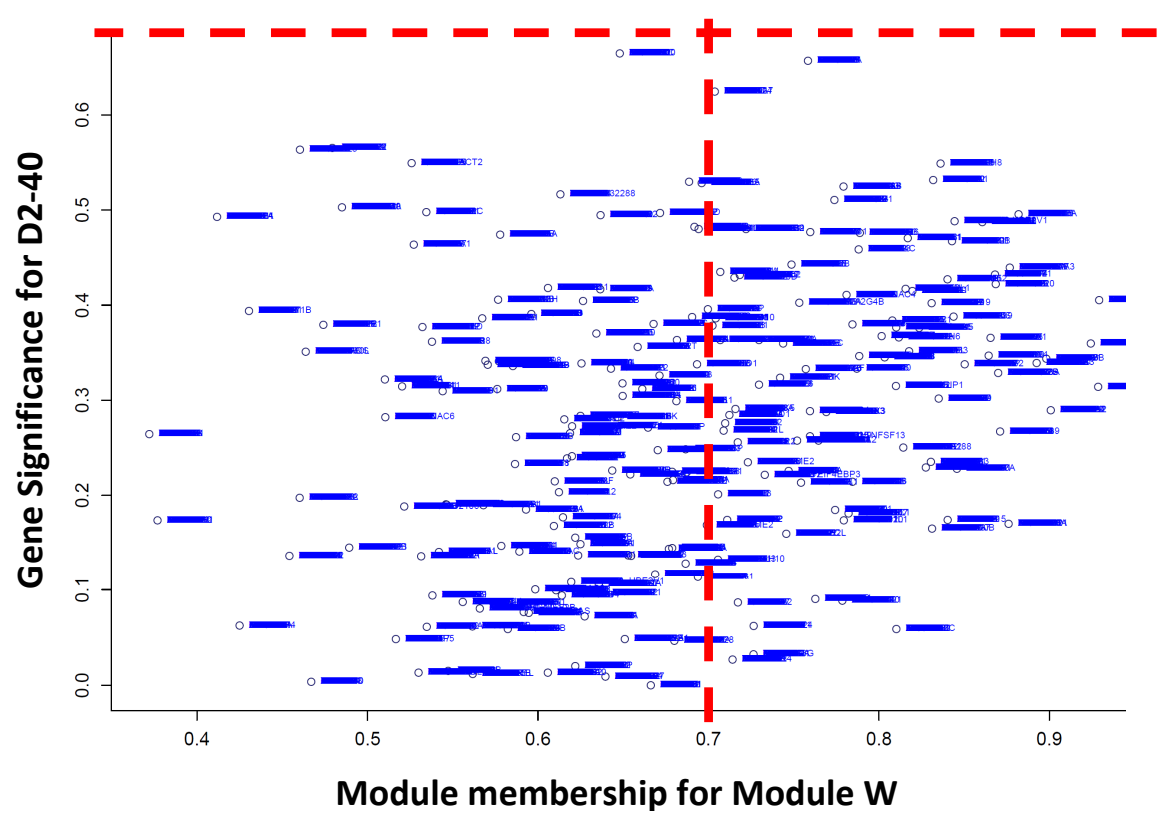

Supplemental Figure V. Module Membership for Module R versus Gene Significance for D2-40. 


\section{References}

1. Doyle B, Caplice N. Plaque neovascularization and antiangiogenic therapy for atherosclerosis. J Am Coll Cardiol. 2007;49:2073-2080

2. Moreno PR, Purushothaman $M$, Purushothaman KR. Plaque neovascularization: Defense mechanisms, betrayal, or a war in progress. Ann N Y Acad Sci. 2012;1254:7-17

3. Sluimer JC, Daemen MJ. Novel concepts in atherogenesis: Angiogenesis and hypoxia in atherosclerosis. J Pathol. 2009;218:7-29

4. Sluimer JC, Kolodgie FD, Bijnens AP, et al. Thin-walled microvessels in human coronary atherosclerotic plaques show incomplete endothelial junctions relevance of compromised structural integrity for intraplaque microvascular leakage. J Am Coll Cardiol. 2009;53:1517-1527

5. Moreno PR, Purushothaman KR, Sirol M, et al. Neovascularization in human atherosclerosis. Circulation. 2006;113:2245-2252

6. Drozdz K, Janczak D, Dziegiel $\mathrm{P}$, et al. Adventitial lymphatics and atherosclerosis. Lymphology. 2012;45:26-33

7. Kholova I, Dragneva G, Cermakova P, et al. Lymphatic vasculature is increased in heart valves, ischaemic and inflamed hearts and in cholesterol-rich and calcified atherosclerotic lesions. Eur J Clin Invest. 2011;41:487-497

8. Alitalo K, Carmeliet P. Molecular mechanisms of lymphangiogenesis in health and disease. Cancer Cell. 2002;1:219-227

9. Carmeliet P. Angiogenesis in health and disease. Nat Med. 2003;9:653-660

10. Carmeliet P, Jain RK. Angiogenesis in cancer and other diseases. Nature. 2000;407:249-257

11. Potente $M$, Gerhardt $H$, Carmeliet $P$. Basic and therapeutic aspects of angiogenesis. Cell. 2011;146:873-887

12. Pugh CW, Ratcliffe PJ. Regulation of angiogenesis by hypoxia: Role of the hif system. Nat Med. 2003;9:677-684

13. Pouyssegur J, Dayan F, Mazure NM. Hypoxia signalling in cancer and approaches to enforce tumour regression. Nature. 2006;441:437-443

14. Poellinger L, Johnson RS. Hif-1 and hypoxic response: The plot thickens. Curr Opin Genet Dev. 2004;14:81-85

15. Carmeliet P, Jain RK. Molecular mechanisms and clinical applications of angiogenesis. Nature. 2011;473:298-307

16. Duff SE, Li C, Garland JM, et al. Cd105 is important for angiogenesis: Evidence and potential applications. Faseb J. 2003;17:984-992

17. Mazzone $M$, Dettori $D$, Leite de Oliveira $R$, et al. Heterozygous deficiency of phd2 restores tumor oxygenation and inhibits metastasis via endothelial normalization. Cell. 2009;136:839-851

18. Gridley T. Vascular biology: Vessel guidance. Nature. 2007;445:722-723

19. Deryugina El, Quigley JP. Pleiotropic roles of matrix metalloproteinases in tumor angiogenesis: Contrasting, overlapping and compensatory functions. Biochim Biophys Acta. 2010;1803:103-120 
20. Pardali E, Goumans MJ, ten Dijke P. Signaling by members of the tgf-beta family in vascular morphogenesis and disease. Trends Cell Biol. 2010;20:556567

21. Augustin HG, Breier G. Angiogenesis: Molecular mechanisms and functional interactions--2nd kloster seeon meeting of the german priority research grant "angiogenesis". Thromb Haemost. 2003;89:190-197

22. Urbich C, Kaluza D, Fromel T, et al. Microrna-27a/b controls endothelial cell repulsion and angiogenesis by targeting semaphorin 6a. Blood. 2012;119:1607-1616

23. Wang $S$, Aurora $A B$, Johnson $B A$, et al. The endothelial-specific microrna mir126 governs vascular integrity and angiogenesis. Dev Cell. 2008;15:261-271

24. Lohela $M$, Saaristo $A$, Veikkola $T$, et al. Lymphangiogenic growth factors, receptors and therapies. Thromb Haemost. 2003;90:167-184

25. Carmeliet $P$, Jain RK. Principles and mechanisms of vessel normalization for cancer and other angiogenic diseases. Nat Rev Drug Discov. 2011;10:417-427

26. Vuorio T, Jauhiainen S, Yla-Herttuala S. Pro- and anti-angiogenic therapy and atherosclerosis with special emphasis on vascular endothelial growth factors. Expert Opin Biol Ther. 2012;12:79-92

27. De Bock K, Cauwenberghs S, Carmeliet P. Vessel abnormalization: Another hallmark of cancer? Molecular mechanisms and therapeutic implications. Curr Opin Genet Dev. 2011;21:73-79

28. Ghilardi C, Chiorino G, Dossi R, et al. Identification of novel vascular markers through gene expression profiling of tumor-derived endothelium. BMC Genomics. 2008;9:201

29. Anfosso L, Efferth T, Albini A, et al. Microarray expression profiles of angiogenesis-related genes predict tumor cell response to artemisinins. Pharmacogenomics J. 2006;6:269-278

30. Hardwick JS, Yang Y, Zhang C, et al. Identification of biomarkers for tumor endothelial cell proliferation through gene expression profiling. Mol Cancer Ther. 2005;4:413-425

31. Donnem T, Fenton CG, Lonvik K, et al. Microrna signatures in tumor tissue related to angiogenesis in non-small cell lung cancer. PLOS One. 2012;7:e29671

32. Virmani R, Kolodgie FD, Burke AP, et al. Lessons from sudden coronary death: A comprehensive morphological classification scheme for atherosclerotic lesions. Arterioscler Thromb Vasc Biol. 2000;20:1262-1275

33. van der Loos CM. Multiple immunoenzyme staining: Methods and visualizations for the observation with spectral imaging. J Histochem Cytochem. 2008;56:313-328

34. Team RdC. R: A language and environment for statistical computing. 2010

35. Du P, Kibbe WA, Lin SM. Lumi: A pipeline for processing illumina microarray. Bioinformatics. 2008;24:1547-1548

36. Langfelder $P$, Horvath S. Wgcna: An $r$ package for weighted correlation network analysis. BMC Bioinformatics. 2008;9:559

37. Ray SS, Bandyopadhyay S, Pal SK. Gene ordering in partitive clustering using microarray expressions. J Biosci. 2007;32:1019-1025 
38. Langfelder P, Zhang B, Horvath S. Defining clusters from a hierarchical cluster tree: The dynamic tree cut package for r. Bioinformatics. 2008;24:719-720

39. Langfelder $P$, Horvath $S$. Eigengene networks for studying the relationships between co-expression modules. BMC Syst Biol. 2007;1:54

40. Langfelder $\mathrm{P}$, Luo $\mathrm{R}$, Oldham $\mathrm{MC}$, et al. Is my network module preserved and reproducible? PLoS Comput Biol. 2011;7:e1001057

41. Shannon P, Markiel A, Ozier O, et al. Cytoscape: A software environment for integrated models of biomolecular interaction networks. Genome Res. 2003;13:2498-2504

42. Bindea G, Mlecnik B, Hackl H, et al. Cluego: A cytoscape plug-in to decipher functionally grouped gene ontology and pathway annotation networks. Bioinformatics. 2009;25:1091-1093

43. Jain RK. Molecular regulation of vessel maturation. Nat Med. 2003;9:685-693

44. Weigand JE, Boeckel JN, Gellert P, et al. Hypoxia-induced alternative splicing in endothelial cells. PLoS One. 2012;7:e42697

45. Spiegel I, Salomon D, Erne B, et al. Caspr3 and caspr4, two novel members of the caspr family are expressed in the nervous system and interact with pdz domains. Mol Cell Neurosci. 2002;20:283-297

46. Da Silva JS, Medina M, Zuliani C, et al. Rhoa/rock regulation of neuritogenesis via profilin iia-mediated control of actin stability. J Cell Biol. 2003;162:12671279

47. Quaegebeur A, Lange C, Carmeliet P. The neurovascular link in health and disease: Molecular mechanisms and therapeutic implications. Neuron. 2011;71:406-424

48. Dallas NA, Samuel S, Xia L, et al. Endoglin (cd105): A marker of tumor vasculature and potential target for therapy. Clin Cancer Res. 2008;14:19311937

49. Hao NB, Lu MH, Fan YH, et al. Macrophages in tumor microenvironments and the progression of tumors. Clin Dev Immunol. 2012;2012:948098

50. Nucera S, Biziato D, De Palma M. The interplay between macrophages and angiogenesis in development, tissue injury and regeneration. Int J Dev Biol. 2011;55:495-503

51. Piehler A, Kaminski WE, Wenzel JJ, et al. Molecular structure of a novel cholesterol-responsive a subclass abc transporter, abca9. Biochem Biophys Res Commun. 2002;295:408-416

52. Chen LJ, Lim SH, Yeh YT, et al. Roles of micrornas in atherosclerosis and restenosis. J Biomed Sci. 2012;19:79

53. Cummins JM, He Y, Leary RJ, et al. The colorectal micrornaome. Proc Natl Acad Sci U S A. 2006;103:3687-3692

54. Bos FL, Caunt M, Peterson-Maduro J, et al. Ccbe1 is essential for mammalian lymphatic vascular development and enhances the lymphangiogenic effect of vascular endothelial growth factor-c in vivo. Circ Res. 2011;109:486-491

55. Habeck $\mathrm{H}$, Odenthal J, Walderich B, et al. Analysis of a zebrafish vegf receptor mutant reveals specific disruption of angiogenesis. Curr Biol. 2002;12:14051412 


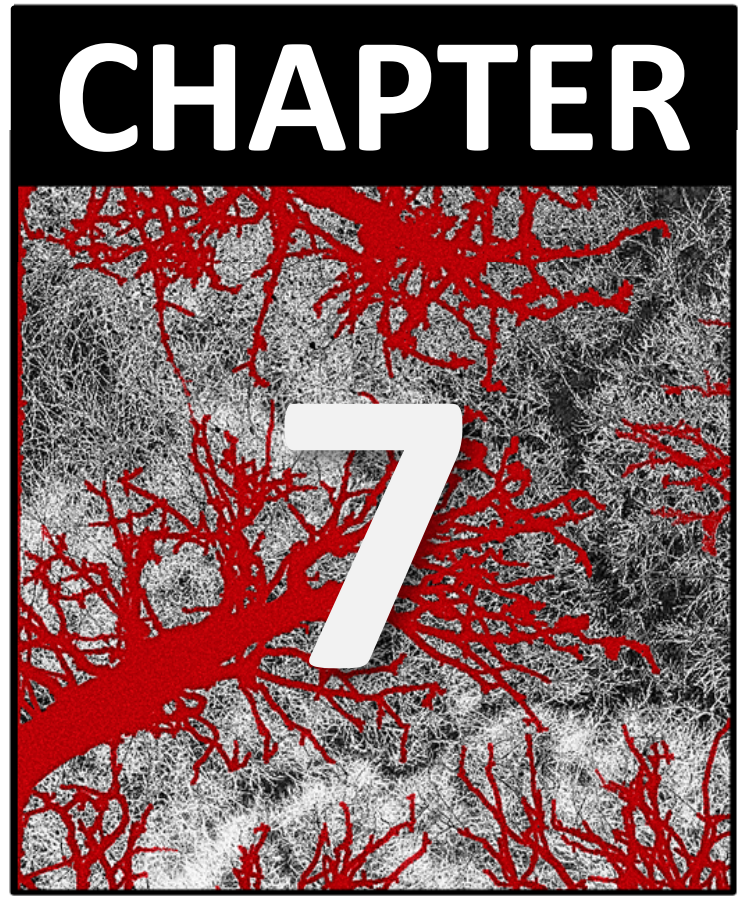




\section{Increased adventitial lymphatic capillaries in atherosclerosis: a path for $T$ cell trafficking?}

Isabelle T.M.N. Daissormont, Timo Rademakers, Jeroen J.T. Otten, Andrey Anisimov, Harri Nurmi, Marc A.M.J. van Zandvoort, Sylvia Heeneman, Kari Alitalo, and Erik A.L. Biessen 


\section{Abstract}

During plaque progression, the adventitia has shown to be progressively enriched in inflammatory cells like $T$ and $B$ cells, mast cells and dendritic cells, cytokines and chemokines, as well as vasa vasorum and lymphatic capillaries. Lymphatic capillaries play an important role in lymphocyte and dendritic cell trafficking as well as analyte communication between inflammatory sites and draining lymph nodes. The observation that the adventitia of human atherosclerotic vessels is enriched not only in T cells but also in lymphatic capillaries has inspired us to address the role of these lymphatic capillaries in regulating $T$ cell influx to, and efflux from the adventitia of atherosclerotic lesions of $\mathrm{ApoE}^{-/-}$mice.

Intravenous administration of $\mathrm{CD}^{+} \mathrm{T}$ cells resulted in a CXCR3 dependent accumulation of $\mathrm{CD}^{+} \mathrm{T}$ cells in the adventitia of the atherosclerotic vessel wall, at which $T$ cell presence displayed an overt gradient from adventitia (high) to intima (scarce). Second, we showed that, as in humans, the lymphatic capillary density in adventitia expanded during murine plaque development. Removal of the draining lymph node and its efferent lymphatic vessel resulted in an increase in plaque volume, accompanied by increased plaque and adventitial $\mathrm{CD}^{+} \mathrm{T}$ cell numbers. Inhibition of lymphangiogenesis using an AAV-hVEGFR3-Ig construct also resulted in $\mathrm{CD}^{+}{ }^{+}$cells enrichment in both plaque and adventitia.

Taken together, this study showed that local lymph capillaries in the adventitia of atherosclerotic plaques may be involved in the dynamics of local $T$ cell efflux and may affect plaque phenotype. 


\section{Introduction}

One of the key processes in atherosclerosis development, both in humans and experimental atherosclerosis models, is the local inflammatory response which drives disease progression. A myriad of immune cell types, e.g. macrophages, $T$ and $B$ cells, dendritic cells (DCs), have been described to be involved in plaque development and/or progression ${ }^{1-5}$. Although the inflammatory response in atherosclerosis is to a large part confined to the lesion itself, there is growing evidence for a crucial role of the adventitia in vascular inflammation and plaque development ${ }^{\text {reviewed in } 6 \text {. }}$

The adventitia is the outer layer of the vessel wall and is a highly organized tissue characterized by stromal tissue, vasculature and the presence of several leukocyte subsets. The adventitia is enriched in B cells, which were shown to exert both pro- and anti-atherogenic effects ${ }^{7-9}$. Moreover, adventitial tissue is a major site of $T$ cell accumulation in mouse atherosclerotic vessels ${ }^{10,11}$. Regulatory $T$ cells and mast cells are scarcely present in the intima, but more abundant in the adventitia ${ }^{12,13}$. The overt presence of DCs in clusters near $\mathrm{T}$ cells in the adventitia, suggest a role for the adventitia as a platform for antigen presentation ${ }^{9,14}$. In addition, the adventitia is a highly vascularized tissue. Vasa vasorum in fact expand with atherosclerosis progression both in human atherosclerosis and murine atherosclerosis models, and is regarded to have an important function in trafficking of inflammatory cells ${ }^{15,16}$.

The adventitia is therefore increasingly viewed as an important gateway for leukocytes to the plaque, a notion referred to as the "outside-in" hypothesis ${ }^{17}$. Findings by Ley and co-workers support the notion that $\mathrm{T}$ cells can enter atherosclerotic lesions via the adventitia ${ }^{11}$. In addition, Eriksson et al further substantiated this finding by demonstrating that leukocytes invade the plaque in an L- and P-selectin dependent manner via microvessels sprouting from the adventitia ${ }^{15}$. Previous work from our group has already shown that the extravasation of inflammatory cells from the vasa vasorum in mice is substantial ${ }^{18}$. Next to vasa vasorum expansion, presence of lymphatic capillaries has recently been demonstrated in the adventitia of human atherosclerotic vessels ${ }^{19,20}$, and the number of adventitial lymphatic capillaries has been shown to increase with severity of atherosclerosis in humans ${ }^{22,23}$. The lymphatic system is also well known for its role in cell trafficking in and out 


\section{CHAPTER 7}

of tissues ${ }^{21-23}$. Although a functional role of adventitial lymphatic capillaries in atherosclerosis has not yet been documented, they conceivably are involved in the regulation of inflammation, by draining (i) antigen-presenting cells from the site of inflammation to the draining lymph node, where lymphocytes will be sensitized and activated to participate in the ongoing inflammatory response; (ii) excess inflammatory cells in general; and/or (iii) secreted growth factors, cytokines, and built-up interstitial fluid.

In this study, we addressed the role of adventitial lymphatic capillaries in a murine model of atherosclerosis by a loss-of-function approach. Our results suggest that the lymphatic capillaries are responsible for drainage of $T$ cells from the atherosclerotic lesion. 


\section{Methods}

\section{Analysis of adventitial lymphatic capillaries and $\mathrm{CD3}^{+} \mathrm{T}$ cells}

Male $\mathrm{ApoE}^{-/}$mice $(\mathrm{n}=21)$, backcrossed at least 11 times to $\mathrm{C} 57 \mathrm{BI} / 6$ and obtained from The Jackson Laboratory, were placed on western type diet (WTD) containing $0.25 \%$ cholesterol and $15 \%$ cacao butter (Special Diets Services, Witham, Essex, UK). Atherosclerotic lesions were induced in the carotid artery by bilateral placement of semi-constrictive collars ${ }^{24}$. To study the expression of lymphatic capillaries and $\mathrm{CD}^{+} \mathrm{T}$ cells in the adventitia of early lesions, mice $(n=9)$ were sacrificed 4 weeks after collar placement. The expression of lymphatic capillaries and $\mathrm{CD}^{+} \mathrm{T}$ cells in the adventitia of advanced lesions was studied in mice $(n=6)$, sacrificed 8 weeks after collar placement. ApoE ${ }^{-/-}$mice without collar placement $(n=6)$ served as control. All animal experiments were approved by the local animal ethics committee and performed according to institutional guidelines.

\section{T cell trafficking experiments}

For T cell trafficking studies, $\mathrm{CD}^{+} \mathrm{T}$ cells were isolated from spleen of wild-type and $\mathrm{CXCR3}^{-1-24}$ mice using a CD3 isolation kit (BD Biosciences) and were prestimulated with $1 \mathrm{ng} / \mathrm{mL} \mathrm{IL-2} \mathrm{(Sigma-Aldrich).} \mathrm{Male} \mathrm{ApoE}^{-/-}$mice $(n=8)$ were placed on western type diet and received a semi-constrictive collar to induce plaque formation. Additionally, these mice received an intravenous injection containing a 1:1 mixture of CMFDA labeled WT ( $10^{7}$ cells) and CMTPX labeled $\mathrm{CXCR3}^{-/-}\left(10^{7}\right.$ cells $)$ T cells 4 weeks after collar placement; controls received WT $\mathrm{T}$ cells only. Twenty-four hours later, mice were sacrificed and perfused with phosphate buffered saline (PBS). The right carotid artery was removed, embedded in Tissue Tek (Miles Inc) and snap-frozen at $-196^{\circ} \mathrm{C}$. Frozen sections $(5 \mu \mathrm{m})$ were cut over a segment covering the entire plaque and nuclei were counterstained with DAPI (Sigma-Aldrich). Overlay images were acquired using a Leica DM5000B fluorescence microscope, using a DAPI filter (DAPI), a FITC filter (CMFDA) and a Tx2 filter (CMTPX). Analysis was performed in a blinded manner. The number of $T$ cells present in the adventitia was expressed per $10 \mu \mathrm{m}$ segment plaque. 


\section{Lymph node and vessel dissection in mice}

Male $\mathrm{ApoE}^{-/-}$mice $(n=27)$ were placed on western type diet and semiconstrictive collars were placed around the carotid arteries to induce atherosclerosis development ${ }^{24}$. To address the contribution of the deep cervical lymph node to leukocyte influx into, and efflux from the adjacent plaque, the lymph node and efferent lymph vessel were removed immediately after collar placement $(n=14)$. Mock treated mice $(n=13)$ served as control. At sacrifice, mice from the lymph node dissection group did not show any signs of edema in the neck region. In a separate study, we investigated whether lymph node and vessel dissection per se could induce an inflammatory response, which could impact lesion formation indirectly. We studied effects of the above intervention in normolipidemic wild-type mice $(n=5-6)$. In both experiments mice were sacrificed 4 weeks after collar placement.

\section{Inhibition of lymphangiogenesis in mice}

Male $\mathrm{ApoE}^{-/-}$mice $(n=34)$ were placed on western type diet and semiconstrictive collars were placed around the carotid arteries to induce atherosclerosis development ${ }^{24}$. To inhibit VEGFR3-dependent adventitial lymphangiogenesis during plaque development, adeno-associated virus (AAV) encoding a soluble hVEGFR3-Ig (AAV-hVEGFR3-lg, $10^{11}$ virus particles per mouse ${ }^{25}$ ) or control virus (AAV-hVEGFR3(D4-7)-Ig) was administered systemically by intravenous injection at the time of collar placement $(n=17$ hVEGFR3-Ig vs. $n=17$ control). Mice were sacrificed 4 weeks after collar placement.

A subgroup of mice ( $n=5$ per group) also received a subcutaneous VEGF-C supplemented matrigel plug (BD Bioscience), as a positive control for inhibition of lymphangiogenesis by the hVEGFR3-Ig construct. In addition, serum from mice was isolated 4 weeks after collar placement by centrifugation of whole blood $\left(10 \mathrm{~min}, 2100 \mathrm{rpm}, 4^{\circ} \mathrm{C}\right)$, and transgene expression was assessed by ELISA and western blot.

\section{Histology of mouse atherosclerotic lesions}

Mice were euthanized by an overdose pentobarbital $(115 \mathrm{mg} / \mathrm{kg})$ and perfused through the left cardiac ventricle with $\mathrm{PBS}\left(\mathrm{NaCl} / \mathrm{Na}_{2} \mathrm{HPO}_{4} / \mathrm{KH}_{2} \mathrm{PO}_{4}, \mathrm{pH}\right.$ 7.4) containing sodium nitroprusside $(0.1 \mathrm{mg} / \mathrm{ml}$, Sigma) and $1 \%$ paraformaldehyde (PFA). The right carotid artery was removed, fixed overnight in 1\% PFA and 
paraffin-embedded sections $(4 \mu \mathrm{m})$ were cut. To determine plaque volume in the carotid artery, sections were stained with haematoxylin and eosin (HE) and plaque area was measured for consecutive cross sections at $100 \mu \mathrm{m}$ intervals over a carotid artery segment that covered the entire plaque. Slides were analyzed in a blinded manner using a Leica DM3000 light microscope (Leica Microsystems) coupled to computerized morphometry (Leica Qwin 3.5.1) ${ }^{26}$.

\section{Immunohistochemistry}

Immunohistochemical (IHC) staining on mouse paraffin carotid artery sections was performed for the lymph vessel markers podoplanin (R\&D systems), LYVE-1 (Abcam), and Prox-1 (Prof. Dr. Alitalo, University of Helsinki, Finland), for T cells using CD3 (DAKO), for macrophages using Mac-3 (BD), and for CXCR3 (Sanbio/MBL) and for its ligand IP-10 (R\&D systems). Lyve- $1^{+}$lymphatic capillary content was expressed as percentage of adventitial area. Adventitial area was defined as the tissue within the outer elastic lamina and twice the smallest intima-media thickness. The number of $\mathrm{CD}^{+}, \mathrm{CXCR}^{+}$and $\mathrm{Mac}-3^{+}$cells was expressed as percentage of total plaque or adventitial cells. IHC staining for podoplanin (R\&D systems) was also performed on paraffin embedded matrigel plugs from the AAV-hVEGFR3-Ig study ( $n=5 /$ group). Lymph vessel number per plug area was determined in three slides per matrigel plug. All slides were analyzed blindly using Leica Qwin software.

\section{Flow cytometry and plasma cholesterol measurements}

Blood, spleen and lymph nodes were removed before perfusion ( $n=10 /$ group) and used for flow cytometry of monocytes (CD11 $b^{\text {high }}$ Ly6G', BD), granulocytes $\left(\mathrm{CD} 11 \mathrm{~b}^{\text {high }}\right.$ Ly6G $\left.^{\text {high }}, \mathrm{BD}\right), \mathrm{T}$ helper cells $\left(\mathrm{CD} 4^{+}, \mathrm{BD}\right)$, effector $\mathrm{T}$ cells $\left(\mathrm{CD} 8 \mathrm{a}^{+}, \mathrm{BD}\right)$ and activated $T$ cells (CD44 ${ }^{\text {high }}$, eBioscience). Briefly spleen and lymph nodes were homogenized, filtered through a $70 \mu \mathrm{m}$ mesh, and subjected to red blood cell lysis. All cells were stained in flow cytometry buffer (1x PBS, 0.5\% BSA, $0.01 \% \mathrm{NaN}_{3}$ ). Staining for blood count analyses was conducted using antibodies to CD45, CD115, Gr1, CD19, and CD3 (all eBiosciences) in Hank's Balanced Salt Solution (HBSS) with $0.3 \mathrm{mM}$ EDTA and $0.1 \%$ BSA. Blood was subjected to red blood cell lysis. Cell counts were estimated utilizing CountBright $^{\mathrm{TM}}$ absolute counting beads (Invitrogen). All flow cytometry analysis was performed on a FACS CANTO II (BD Bioscience). 
Plasma cholesterol levels were measured using a colorimetric assay (CHODPAP, Roche, Mannheim, Germany). Disposables were purchased from Greiner Bio One.

\section{Multiphoton laser scanning microscopy (MPLSM)}

For MPLSM of adventitial lymphatics, $\mathrm{ApoE}^{--}$mice $(n=6)$ were anaesthetized by dorsal subcutaneous injection with ketamin $(100 \mathrm{mg} / \mathrm{kg}$ body weight, Nematek) and xylazin (10 mg/kg body weight, Sedamun). Hereafter, mice were injected with the fluorescently labelled ligands directly into the deep cervical lymph node or intracutaneously above the clavicula (as indicated) and after 10 minutes mice were sacrificed. The lumen of the vasculature was flushed with $0.9 \% \mathrm{NaCl}$ by intracardial perfusion, and carotid arteries were exposed and in situ imaging was performed.

For imaging, the following probes were used: biotinylated podoplanin (R\&D systems) conjugated to Dylight594 (ThermoScientific) for staining the lymphatic endothelial cells, Syto 41 (blue, Invitrogen) for staining all nuclei, a FITC-labelled CABD probe ${ }^{27}$ (green) for tracking lymph vessels in the adventitia, and CMTPX labeled (red, $10^{7}$ cells) T cells. MPLSM imaging was performed on a Leica SP5 imaging platform (Leica Microsystems, Germany) that integrates multiphoton microscopy with fast resonant scanning, and uses a Compact Ultrafast femtosecond-pulsed Ti:Sapphire Laser (Chameleon, Coherent, USA). An excitation wavelength of $880 \mathrm{~nm}$ was used in all experiments. Tissues were observed using a 20x 1.0 water immersion objective (HCX PL APO L, Leica Microsystems, Germany) with a numerical aperture of 1.00 and integrated optical zoom allowing magnification up to 60x. Photo-multiplier tubes were used to detect two spectral regions: $515-560 \mathrm{~nm}$ (FITC, Syto13) and 570$600 \mathrm{~nm}$ (Dylight594, CMTPX). Image acquisition was performed at a frame size of $512 \times 512$ pixels (pixel size: $1.44 \times 1.44 \mu \mathrm{m}$ ), scanning at $100 \mathrm{~Hz}$ and a frame average filter of 2 was used to reduce noise. In-depth scans were performed at $200 \mathrm{~Hz}$ with a frame average of 2 . Consecutive images had an interplanar distance of $1.00 \mu \mathrm{m}$.

\section{Statistical analysis}

Data are expressed as mean \pm SEM and Mann-Whitney $U$ test was used to compare individual groups of animals. Statistics were performed using Graphpad Prism 5.0. Data were considered statistically significant at $p<0.05$. 


\section{Results}

\section{Lymphatic capillaries were present in the adventitia of mouse atherosclerotic} lesions and their presence increased with plaque progression

First, we investigated the presence of lymphatic capillaries in the adventitia of mouse atherosclerotic lesions. In the adventitia, both vasa vasorum and lymphatic capillaries were present (Figure $1 \mathrm{~A}$ ). Concordant with observations in human atherosclerotic lesions ${ }^{19,20}$, we observed that lymphatic capillary numbers in the adventitia were already significantly increased early in plaque development (Figure 1B). However, we did not observe any further expansion of the adventitial lymphatic vessel bed at later stages of plaque development, suggesting that lymphatic expansion occurs already early in plaque development.
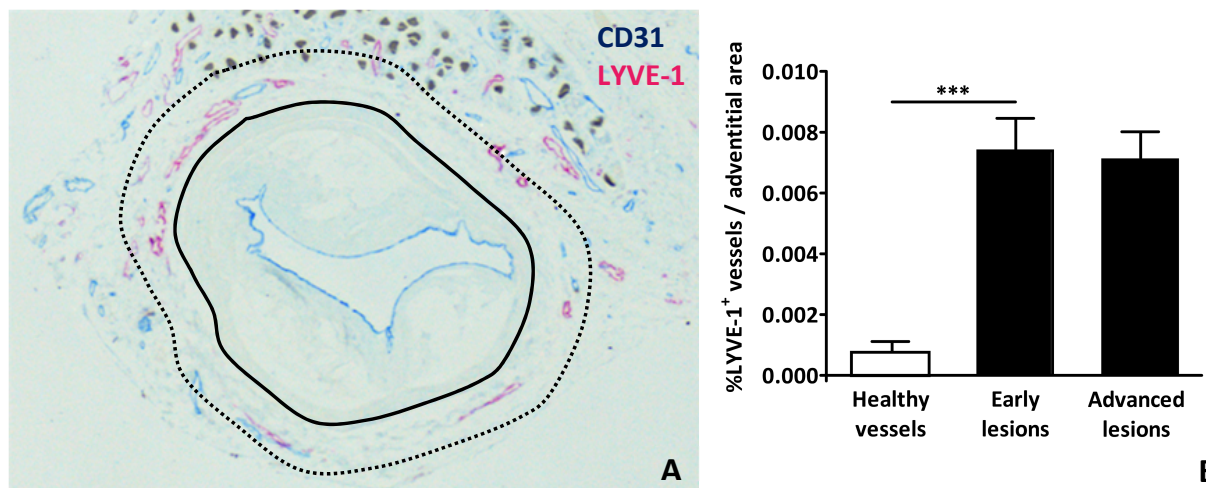

Figure 1. The presence of lymphatic capillaries in the adventitia of atherosclerotic lesions. Immunohistochemistry for $\mathrm{LYVE}-1^{+}$lymphatic capillaries (purple) and $\mathrm{CD} 1^{+}$vasa vasorum (blue) in mouse carotid atherosclerotic lesions (A). Continuous line indicates the outer border of the media; the area between the continuous and dotted line indicates the adventitial area used for quantification. Perivascular fat was excluded from analysis (A). The percentage of LYVE-1 $1^{+}$ lymphatic capillaries per adventitia area of healthy $(n=6)$ versus early $(n=9)$ and advanced $(n=6)$ mouse carotid atherosclerotic lesions (B). ${ }^{* * *} p<0.05$ 


\section{$\mathrm{CD3}^{+} \mathrm{T}$ cells were abundant in the adventitia of mouse atherosclerotic lesions} and entered the vessel wall via the adventitia in a CXCR3 dependent manner Adventitial $T$ cell accumulation started during plaque initiation and decreased with plaque progression (Figure $2 \mathrm{~A}$ ). This observation suggests that $\mathrm{CD}^{+} \mathrm{T}$ cells may invade the vessel wall via the adventitia to contribute to early atherosclerosis development. To address this question, CMFDA (green) labeled $\mathrm{CD}^{+} \mathrm{T}$ cells were administered via intravenous injection in western type diet fed $\mathrm{ApoE}^{-/-}$mice four weeks after collar placement, when early lesion had formed.
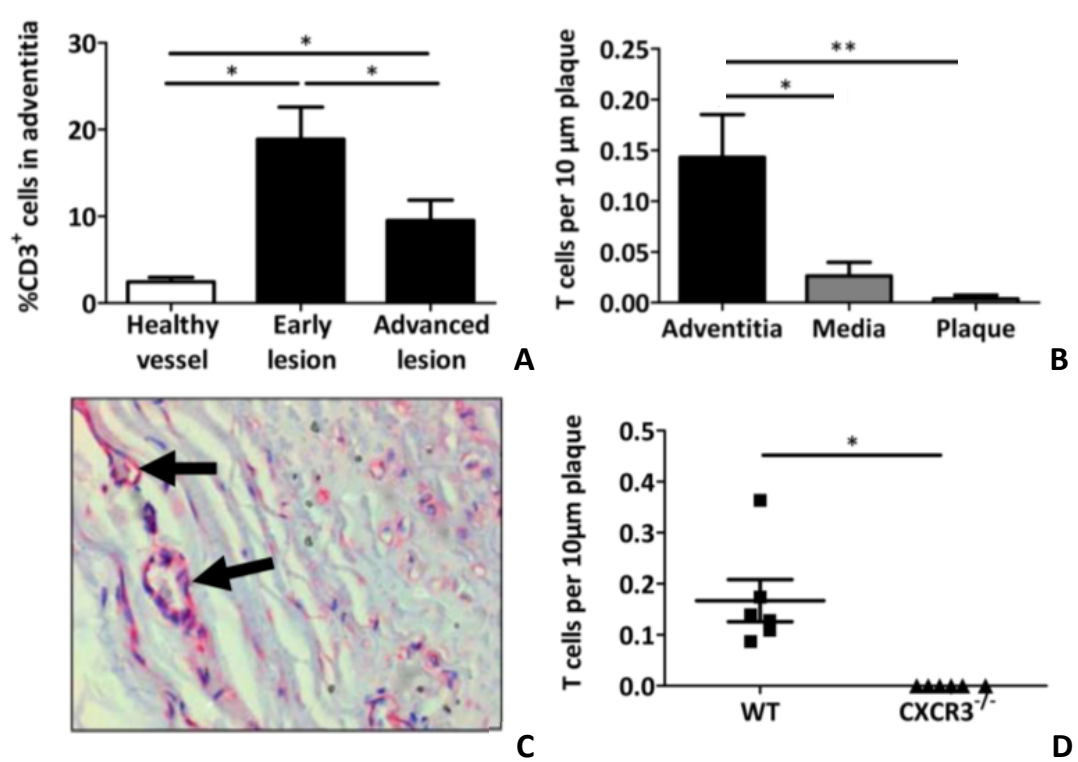

Figure 2. Expression and migration of $\mathrm{CD}^{+} \mathrm{T}$ cells in the adventitia. The percentage of $\mathrm{CD} 3^{+} \mathrm{T}$ cells per adventitia area of healthy vessels $(n=6)$ versus early $(n=9)$ and advanced $(n=6)$ mouse carotid atherosclerotic lesions showed a clear increase during plaque development, especially in early lesions (A). A $C D 3^{+} T$ cells homing experiment showed a gradient of $T$ cells from the adventitia, via media to the plaque within 24 hours after injection, reflective of adventitia to intima $T$ cell flux (B). Immunohistochemistry for IP-10, the main chemokine for attracting activated $T$ cells, on mouse carotid atherosclerotic lesions revealed high IP-10 expression in the adventitia, most notably by vasa vasorum and scattered leukocytes (C). Arrows indicate the expression of the CXCR3 ligand IP-10 by the vasa vasorum. Injection of $\mathrm{CXCR3}^{-1} \mathrm{CD}^{+} \mathrm{T}$ cells showed an abrogated homing of $T$ cells to the adventitia within 24 hours compared to wild-type $C D 3^{+} T$ cells (D), arguing for IP-10/CXCR3-dependent $T$ cell homing. ${ }^{*} p<0.05,{ }^{* *} p<0.01$. 
Histological analysis revealed a clear-cut gradient in $\mathrm{CD}^{+} \mathrm{T}$ cell density from the adventitia (high numbers), via the media (intermediate numbers) to the intima (low numbers) (Figure 2B). In search of chemokines that might be responsible for adventitial $T$ cell accumulation, we focused on Interferon Inducing Protein-10 (IP-10), which was highly expressed in the adventitia, especially around the vasa vasorum (Figure 2C, arrows) and is known to interact with CXCR3 as a major mediator of T cell chemotaxis ${ }^{28}$. To investigate whether $T$ cell migration towards the adventitia occurred in a CXCR3 dependent manner, we administered a mixture of CMFDA (green) labeled wildtype and CMTPX (red) labeled $\mathrm{CXCR}^{--} \mathrm{CD}^{+} \mathrm{T}$ cells via intravenous injection into western type diet fed $\mathrm{ApoE}^{-/-}$mice, four weeks after collar placement. In contrast to wild-type $\mathrm{T}$ cells, which were prominently recruited, $\mathrm{CXCR}^{-1-} \mathrm{T}$ cell migration towards the adventitia was almost ablated (Figure 2D), indicating that adventitial $\mathrm{T}$ cell accumulation is largely CXCR3 dependent.
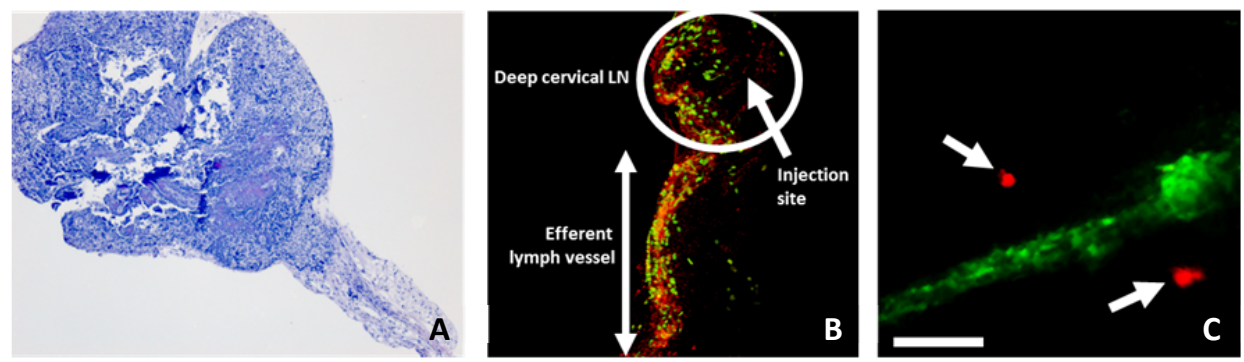

Figure 3. Detection of lymph vessels and capillaries ex vivo and in situ. Dissected lymph node and efferent lymph vessel from lymph node dissection experiments (A). Lymph node and efferent lymph vessels could be stained for podoplanin (red) ex vivo ( $B$, nuclei in green). A lymph-residing FITC-labelled CABD probe allowed staining of adventitial lymph capillaries in situ, and showed close proximity of CMTPX (red) labeled $\mathrm{CD}^{+} \mathrm{T}$ cells to lymph capillaries in ApoE ${ }^{-/-}$mice (C). Bar indicates $25 \mu \mathrm{m}$.

To address the association between $T$ cells and the adventitial lymph capillaries, MPLSM was used to visualize the lymphatic system. Intranodal injection of a podoplanin antibody into the draining lymph node before dissection (Figure $3 A$ ) visualized the lymph node and efferent lymph vessel (Figure 3B, red), but not the lymph capillaries in the adventitia (not shown). Moreover, by concomitant intracutaneous injection of a lymph-residing CABD probe, to visualize lymph capillaries ${ }^{27}$, and intravenous injection of CMTPX labeled $\mathrm{CD}^{+} \mathrm{T}$ cells, we were able to detect $\mathrm{CD}^{+} \mathrm{T}$ cells (Figure $3 \mathrm{C}$, red) in close proximity of the adventitial lymph capillaries (Figure $3 \mathrm{C}$, green). 
Lymph node and lymph vessel dissection deteriorated atherosclerosis development in $\mathrm{ApoE}^{-/-}$mice by promoting $\mathrm{T}$ cell accumulation inside the lesion and adventitia

The proximity of $T$ cells to the adventitial lymph capillaries, together with the fact that lymph vessels have been implicated in regulation of inflammation ${ }^{29,30}$, argue a potential role for lymph capillaries in the inflammatory process in atherosclerotic arteries. To elucidate the role of adventitial lymph capillaries, we studied the effect of dissection of the draining lymph vessels and lymph node. The deep cervical lymph node is proximal to the carotid artery bifurcation. An efferent lymphatic vessel is originating from this lymph node, running upstream parallel to the carotid artery. To address whether adventitial lymph capillaries are involved in leukocyte accumulation and plaque development, the deep cervical lymph node and its efferent vessel were removed during collar placement in $\mathrm{ApoE}^{-/}$mice (Figure 4A).

First, lymph node dissection by itself did not induce local inflammation in the adventitia, as judged the equal $\mathrm{CD}^{+} 5^{+}$leukocyte content between mice that had undergone lymph node dissection (Figure 4B). In addition, circulating monocyte numbers (Figure $4 \mathrm{C}$ ) and spleen and peripheral lymph node $\mathrm{CD}^{+} \mathrm{T}$ cell content (Figure 4D, 4E) were unchanged as well, and lymph node dissection did not impact body weight $(29.23 \pm 0.48 \mathrm{~g}$ and $27.64 \pm 0.52 \mathrm{~g}$ in control versus lymph node dissected mice, respectively). Interestingly, lymph node dissection aggravated atherosclerotic plaque development (Figure 4F, $4 \mathrm{G}$ ) and plaques displayed increased adventitial (Figure $4 \mathrm{H}$ ) and intimal $\mathrm{CD}^{+} \mathrm{T}$ cell content (Figure 4I). Plaque macrophage content remained unaltered (Figure 4J). Remarkably, we did not observe any effects of draining lymph node removal on lymphatic capillary bed density, in the adventitia (Figure 4K) at end point (four weeks after lymph node dissection), although origin and functionality of these newly formed capillaries remained unclear. Altogether, these data point to a role of adventitial lymphatic capillaries in draining $T$ cells and/or $T$ cell activating chemokines and cytokines from the plaque, translating in reduced $\mathrm{T}$ cell but not macrophage accumulation in the plaque. This in turn may dampen inflammation and plaque development. 

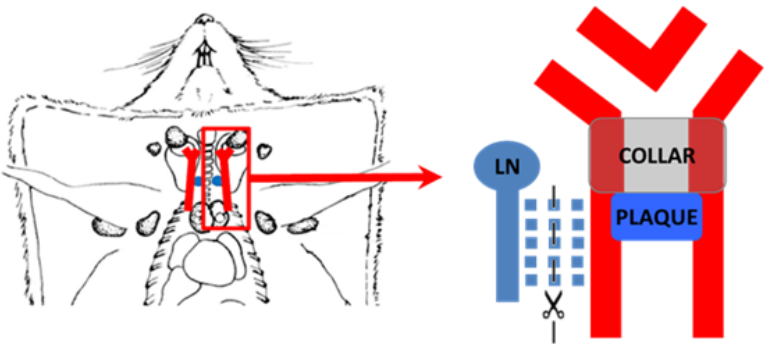

A
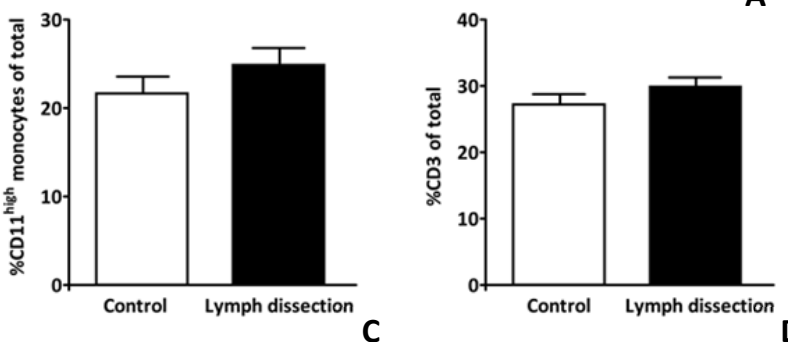

C
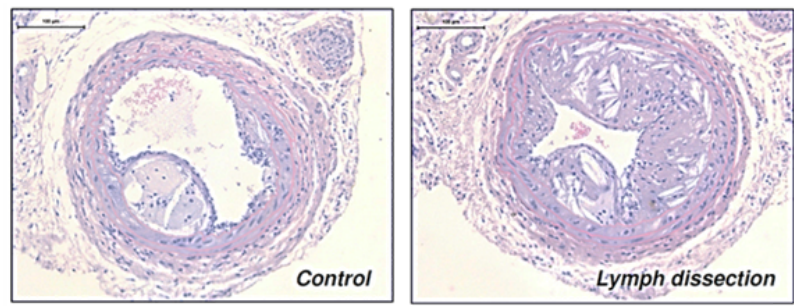

D
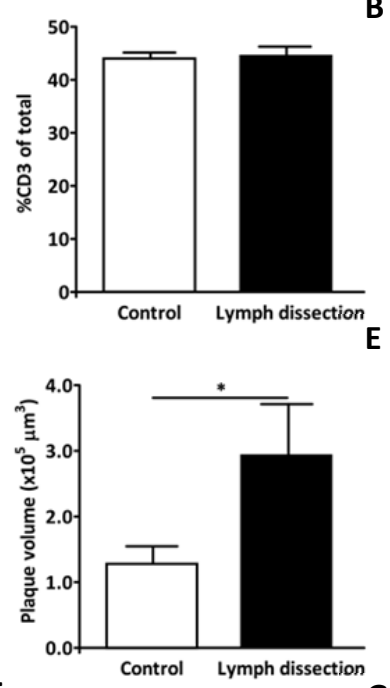

G

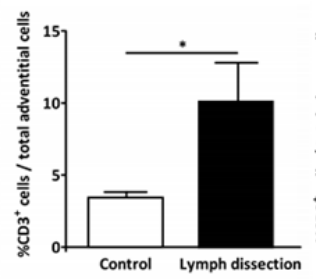

H
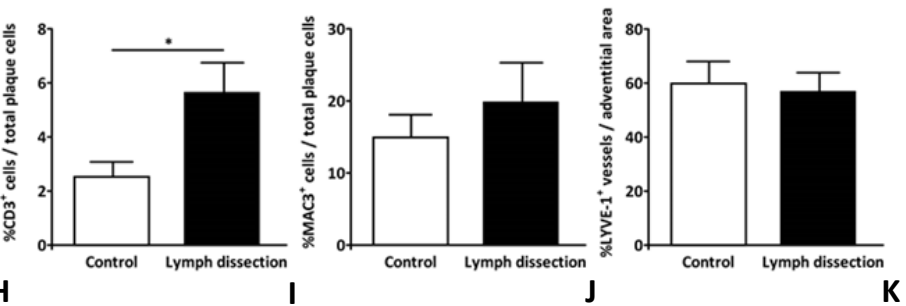

Figure 4. Lymph node dissection results in an aggravation of plaque development in mice, characterized by an accumulation of $\mathrm{CD}^{+} T$ cells within the vessel wall. Draining lymph node and efferent vessel were excised in the lymph node dissection group (A). Lymph node dissection by itself did not induce inflammation in the vessel wall. Represented is the percentage of $C D 45^{+}$ leukocytes in the adventitia of control $(n=6)$ and lymph node dissected $(n=5)$ WT mice (B). Flow cytometry analysis for $C D 11 b^{\text {high }}$ monocytes in blood (C), as well as $C D 3^{+} T$ cells in spleen (D) and peripheral lymph nodes (E) revealed no changes. Lymph node dissection resulted in bigger plaques as observed by HE staining (F). Quantification of plaque volume confirmed the increase in plaque volume in the lymph node dissection group (G). Lymph node dissection increased the percentage of $\mathrm{CD}^{+} \mathrm{T}$ cells in the adventitia $(\mathrm{H})$ and within the plaque (I), while plaque Mac- $3^{+}$macrophage presence was unaltered (J). The percentage of $L Y V E-1^{+}$lymphatic capillary area in the adventitia also was unchanged. ${ }^{*} p<0.05$. 
Inhibition of lymphangiogenesis using an AAV-hVEGFR3-Ig construct did not change plaque burden, yet increased lesional $T$ cell content

The lymph node dissection study suggested that the efferent lymph vessel that parallels the carotid artery is not accountable for plaque initiation associated adventitial lymph vessel expansion. Therefore, we sought to address the effect of systemic interference in lymphangiogenesis by AAV overexpression of IgG fused soluble human VEGFR3 (AAV-hVEGFR3-Ig ${ }^{25}$ ). Gene therapy led to persistent 3-fold overexpression of soluble hVEGR3 in serum of treated mice, but not controls as assessed by western blotting (Figure 5A) and ELISA (Figure $5 B$ ). The achieved sVEGFR3 levels were sufficient to halt, the lymphangiogenic response in an in vivo VEGF-C supplemented matrigel plug assay, as illustrated by the sharp reduction in lymph vessel ingrowth (Figure $5 \mathrm{C}$ ). AAV gene therapy showed a borderline reduction in body weight (Supplemental Figure I-A; $p=0.053$ ), but did not alter plasma cholesterol levels (Supplemental Figure IB), nor did it affect leukocyte subset counts and distribution in blood, spleen, and lymph nodes, except for a tendency towards decreased numbers of CD4 ${ }^{+}$Ly6C $C^{\text {high }}$ effector-memory $T$ helper cells in the draining deep cervical lymph node, accompanied by an increase in $C D 4^{+} L y 6 C^{\text {low }}$ naïve $T$ cells (not shown).

Plaque volume (Figure 5D) and lipid core volume (Supplemental Figure I-C) were not significantly affected. However, plaques of AAV-hVEGFR3-Ig treated mice showed a more inflammatory phenotype, with increased adventitial and intimal $\mathrm{CD}^{+} \mathrm{T}$ cell content (Figure $5 \mathrm{E}$ ). Much to our surprise, while halting systemic lymphangiogenesis in the matrigel plug assay, hVEGFR3 overexpression failed to inhibit adventitial lymphatic capillary expansion (Figure 5F). Moreover, in advanced inflammatory plaques, we observed an increase of lymph capillaries inside the plaque (Figure $5 \mathrm{G}, 5 \mathrm{H}$ ).

Collectively, in both the systemic lymphangiogenesis intervention study and the LN dissection study, we observed a striking increment in lesional $\mathrm{T}$ cell content, pointing to a role of plaque associated lymph vessels in regulating local $\mathrm{T}$ cell content. 

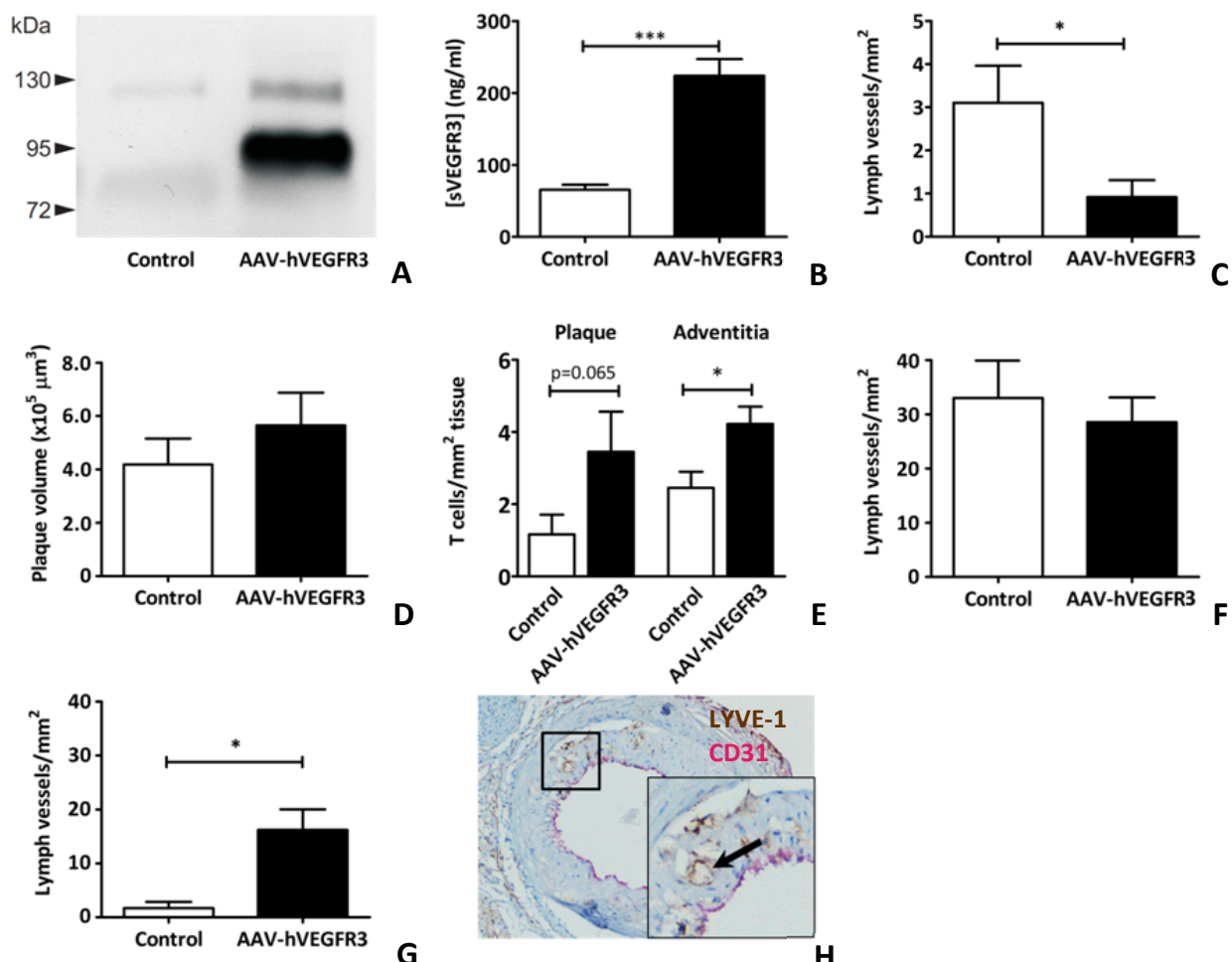

G

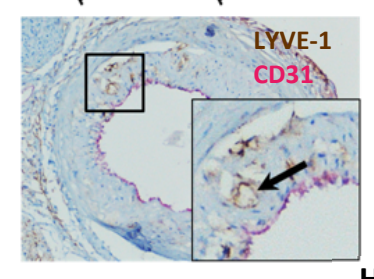

Figure 5. Soluble hVEGFR3 gene therapy led to effective inhibition of systemic but not plaqueassociated lymphangiogenesis, yet induced an increase in plaque $T$ cell content. Lymphangiogenesis was inhibited by intravenous AAV-hVEGFR3-Ig gene transfer resulting in a persistent, clear expression of soluble hVEGFR3, detected by western blotting (A), and an increase in total soluble hVEGFR3 (ELISA) in plasma (B). AAV-hVEGFR3-Ig gene transfer reduced lymph vessel formation and ingrowth in an in vivo matrigel plug assay ( $C, n=5$ mice/group) four weeks after s.c. administration. While plaque volume was not affected by AAV-hVEGFR3 treatment (D), it did significantly increase adventitial and plaque $T$ cell content (E). Remarkably, the lymph vessel density was unaltered in the adventitia at point of sacrifice (F), and an increased lymph vessel density was observed within the plaque (G), as visualized by LYVE-1 ${ }^{+}$staining $(H$, arrow). ${ }^{*} p<0.05,{ }^{* * *} p<0.005$ 


\section{Discussion}

In this study, we are the first to describe a role for adventitial lymphatic capillaries in plaque inflammation and atherosclerosis in mice. We show that the adventitial lymphatic capillary bed is expanded early on in the atherogenic process, concomitantly with the increase in plaque $\mathrm{CD}^{+}{ }^{+}$cell content. $\mathrm{CD}^{+} \mathrm{T}$ cells were found to enter the atherosclerotic vessel wall in a CXCR3 dependent manner, mainly via the adventitia. Combined dissection of the plaque draining lymph node and its efferent lymphatic vessel prior to the atherogenic stimulus resulted in an aggravation of atherosclerosis development, in which lesions were characterized by increased T cell accumulation in plaque and adventitia. Augmented accumulation of T cells was also found after systemic inhibition of VEGFR3 dependent lymphangiogenesis. Paradoxically, both interventions did not impact the lymph capillary bed density in adventitia and plaque, suggesting that atherosclerosis-related interstitial pressure build-up and/or inflammation may be the driving forces in plaque lymphangiogenesis.

An intriguing finding was that during atherosclerosis in $\mathrm{ApoE}^{-/-}$mice the adventitial lymphatic capillary bed is expanded, already at early stages of disease development. These data correspond with previous findings in humans in which the lymphatic capillary content of carotid artery plaques was seen to increase with severity of atherosclerosis ${ }^{19,20}$. Although the exact trigger for adventitial lymphangiogenesis during atherosclerosis remains to be established, it is conceivable that it is related to the augmented production of growth factors such as VEGFs and pro-lymphangiogenic cytokines/chemokines in the inflamed plaque and adventitia ${ }^{31}$. VEGF expression in the atherosclerotic vessel wall has been documented in several studies and its overexpression was shown to accelerate atherosclerosis by inducing monocyte activation, adhesion and migration and by enhancing vascular permeability ${ }^{32-34}$. In addition to VEGFs, various chemokines have been shown to regulate lymphangiogenesis. CCL21, which is expressed in the adventitia, and its receptor CCR7 were both reported to be involved in the induction of lymphangiogenesis ${ }^{35,36}$. A similar regulatory role in lymphangiogenesis and lymph vessel homeostasis was proposed for the CXCL13/CXCR5 axis and the CXCL12/CXCR4 axis ${ }^{37-39}$. Altogether, these data show a link between adventitial lymphangiogenesis and inflammation. 
Our data showed that, concordant with previous observations ${ }^{11,17}$, the adventitia of mouse atherosclerotic lesions expresses high numbers of $\mathrm{CD3}^{+} \mathrm{T}$ cells, where the adventitia contains much more T cells than the intima. In fact, adoptively transferred $T$ cells migrate primarily to the adventitia, then to the media and to a lesser extent to the intima, suggestive adventitial to intimal flux. T cell influx into the adventitia is CXCR3 dependent. The main chemokine to activate CXCR3 and attract activated T cells is IP-10. It is secreted by activated $T$ cells, fibroblasts and endothelial cells ${ }^{28,40,41}$, and is as we show highly expressed in the adventitia of atherosclerotic vessels.

Given the (putative) role of lymphatic vessels in dendritic cell trafficking to the draining lymph node to activate $\mathrm{T}_{\text {cells }}{ }^{29}$, we hypothesized that interruption of lymph draining by lymph node and efferent vessel dissection would result in impaired T cell activation and consequently reduced plaque inflammation. Surprisingly, removal of the plaque draining lymph node located proximal to the carotid artery bifurcation, resulted in aggravation of atherosclerosis, featured by pronounced $\mathrm{CD}^{+} \mathrm{T}$ cell accumulation in plaque and adventitia. Taken together with the close proximity of $T$ cells to the adventitial lymph capillaries, our data suggest that the perivascular lymphatic bed in the adventitia may well be involved in drainage of T cells, and thus may play a role in the regulation of inflammation in the vessel wall. In addition to T cells, adventitial lymphatic capillaries could also help to prevent edema, inflicted by the leaky plaque vasa vasorum, drain plaque cytokines and in this way regulate inflammatory responses, and mediate efflux of lipid particles ${ }^{42}$. Further study is required to dissect the contribution of these factors.

Interestingly, inhibition of lymphangiogenesis did, in contrast to lymph node and efferent lymph vessel dissection, not affect atherosclerotic burden, although it was also seen to increase adventitial and plaque $\mathrm{CD}^{+} \mathrm{T}$ cell content. The exact mechanisms of $T$ cell accumulation remain elusive, but could be attributable to initial impairment of $T$ cell drainage from the plaque, after systemic hVEGFR3-dependent lymphangiogenesis blockage. The resulting reduced draining capacity may elicit an alternative VEGF-C and -D independent lymphangiogenesis axis in plaque tissue, such as the mechano-induced integrin pathway recently described by Planas-Paz et al ${ }^{43}$, or the CXCL12-CXCR4 pathway, which earlier was shown to confer atheroprotection 38,44 . Unpublished work from our group has demonstrated high CXCR4 expression in atherosclerotic lesions, representing/harboring lymphangiogenic potential. 


\section{CHAPTER 7}

Such alternative VEGFR3-independent pathways may explain the unimpeded lymph vessel density in the adventitia and overt presence of lymph vessels in the atherosclerotic lesion itself observed after hVEGFR3 intervention. Future studies could interrogate this notion by measuring the interstitial pressure in the plaque and intraplaque $\beta_{1}$-integrin activation as a measure of mechanoinduced lymphangiogenesis, or by co-inhibition of the CXCL12-CXCR4 axis.

The interpretation of our findings is further complicated by the reciprocal interaction between lymph vessels and $T$ cells. Besides the effect lymph vessels have on T cell trafficking, as illustrated in this study, T cells were vice versa also reported to inhibit lymphangiogenesis. Our current explorative study does not address the relevance of cross-interaction under pathologic conditions and further studies will be needed to gain further insight into the interactions between the two. Of special interest would in this regard be the role of the T cell subset polarization, since e.g. Th1 and Th2 cells are associated with expression of different cytokine and inflammatory mediator repertoires ${ }^{45}$.

In conclusion, this study shows that adventitial lymphatic vessels increased at an early stage of plaque development. Draining lymph node dissection and inhibition of lymphangiogenesis resulted in aggravation of atherosclerosis after lymph node dissection, accompanied by increased plaque and adventitial $\mathrm{T}$ cell content, probably due to disturbed drainage of $\mathrm{T}$ cells. Further studies are needed to give additional insights into the precise role and actual mechanisms of adventitial lymphatic capillary formation in atherosclerosis. 


\section{Supplemental Figures}
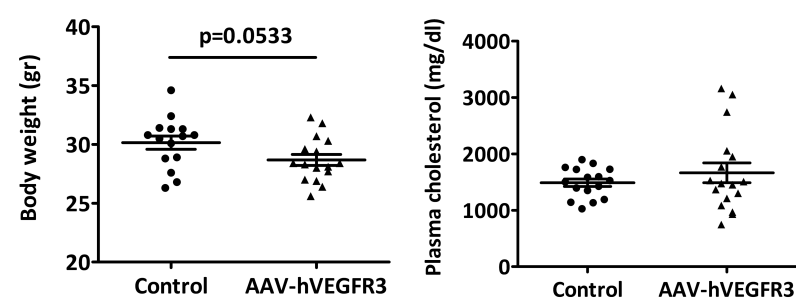

A

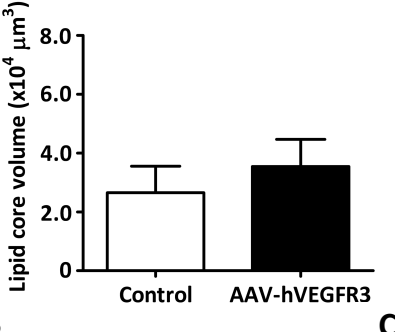

B

Supplemental Figure I. AAV-hVEGFR3-Ig treatment resulted in a slightly reduced body weight, as was described previously ${ }^{25}$ (A). Plasma cholesterol levels were unaltered by AAV-hVEGFR3-Ig treatment $(B)$. In parallel to the unchanged plaque volume, lipid core volume was not different from control mice after AAV-hVEGFR3-Ig treatment (C). 


\section{References}

1. Weber C, Zernecke A, Libby P. The multifaceted contributions of leukocyte subsets to atherosclerosis: Lessons from mouse models. Nat Rev Immunol. 2008;8:802-815

2. Bot I, Biessen EA. Mast cells in atherosclerosis. Thromb Haemost. 2011;106:820-826

3. Hansson GK, Hermansson A. The immune system in atherosclerosis. Nat Immunol. 2011;12:204-212

4. Businaro R, Tagliani A, Buttari B, et al. Cellular and molecular players in the atherosclerotic plaque progression. Ann N Y Acad Sci. 2012;1262:134-141

5. Libby P. Inflammation in atherosclerosis. Arterioscler Thromb Vasc Biol. 2012;32:2045-2051

6. Campbell KA, Lipinski MJ, Doran AC, et al. Lymphocytes and the adventitial immune response in atherosclerosis. Circ Res. 2012;110:889-900

7. Ait-Oufella $\mathrm{H}$, Herbin $\mathrm{O}$, Bouaziz JD, et al. B cell depletion reduces the development of atherosclerosis in mice. J Exp Med. 2010;207:1579-1587

8. Kyaw $\mathrm{T}$, Tipping $\mathrm{P}, \mathrm{Toh} \mathrm{BH}$, et al. Current understanding of the role of $\mathrm{b}$ cell subsets and intimal and adventitial b cells in atherosclerosis. Curr Opin Lipidol. 2011;22:373-379

9. Moos MP, John N, Grabner R, et al. The lamina adventitia is the major site of immune cell accumulation in standard chow-fed apolipoprotein e-deficient mice. Arterioscler Thromb Vasc Biol. 2005;25:2386-2391

10. Zhao L, Moos MP, Grabner R, et al. The 5-lipoxygenase pathway promotes pathogenesis of hyperlipidemia-dependent aortic aneurysm. Nat Med. 2004;10:966-973

11. Galkina E, Kadl A, Sanders J, et al. Lymphocyte recruitment into the aortic wall before and during development of atherosclerosis is partially I-selectin dependent. J Exp Med. 2006;203:1273-1282

12. de Boer OJ, van der Meer JJ, Teeling $\mathrm{P}$, et al. Low numbers of foxp3 positive regulatory $t$ cells are present in all developmental stages of human atherosclerotic lesions. PLoS One. 2007;2:e779

13. Bot I, de Jager SC, Bot $\mathrm{M}$, et al. The neuropeptide substance $\mathrm{p}$ mediates adventitial mast cell activation and induces intraplaque hemorrhage in advanced atherosclerosis. Circ Res. 2010;106:89-92

14. Yilmaz A, Rowley A, Schulte DJ, et al. Activated myeloid dendritic cells accumulate and co-localize with $\mathrm{cd} 3+\mathrm{t}$ cells in coronary artery lesions in patients with kawasaki disease. Exp Mol Pathol. 2007;83:93-103

15. Eriksson EE. Intravital microscopy on atherosclerosis in apolipoprotein edeficient mice establishes microvessels as major entry pathways for leukocytes to advanced lesions. Circulation. 2011;124:2129-2138

16. Langheinrich AC, Michniewicz A, Bohle RM, et al. Vasa vasorum neovascularization and lesion distribution among different vascular beds in apoe-/-/ldl-/- double knockout mice. Atherosclerosis. 2007;191:73-81 
17. Maiellaro K, Taylor WR. The role of the adventitia in vascular inflammation. Cardiovasc Res. 2007;75:640-648

18. Rademakers T, Douma K, Hackeng TM, et al. Plaque-associated vasa vasorum in aged apolipoprotein e-deficient mice exhibit proatherogenic functional features in vivo. Arterioscler Thromb Vasc Biol. 2013;33:249-256

19. Drozdz K, Janczak D, Dziegiel $\mathrm{P}$, et al. Adventitial lymphatics and atherosclerosis. Lymphology. 2012;45:26-33

20. Kholova I, Dragneva G, Cermakova P, et al. Lymphatic vasculature is increased in heart valves, ischaemic and inflamed hearts and in cholesterol-rich and calcified atherosclerotic lesions. Eur J Clin Invest. 2011;41:487-497

21. Girard JP, Moussion C, Forster R. Hevs, lymphatics and homeostatic immune cell trafficking in lymph nodes. Nat Rev Immunol. 2012;12:762-773

22. Na IK, Markley JC, Tsai JJ, et al. Concurrent visualization of trafficking, expansion, and activation of $\mathrm{t}$ lymphocytes and t-cell precursors in vivo. Blood. 2010;116:e18-25

23. Tewalt EF, Cohen JN, Rouhani SJ, et al. Lymphatic endothelial cells - key players in regulation of tolerance and immunity. Front Immunol. 2012;3:305

24. von der Thusen JH, van Berkel TJ, Biessen EA. Induction of rapid atherogenesis by perivascular carotid collar placement in apolipoprotein e-deficient and lowdensity lipoprotein receptor-deficient mice. Circulation. 2001;103:1164-1170

25. Lin J, Lalani AS, Harding TC, et al. Inhibition of lymphogenous metastasis using adeno-associated virus-mediated gene transfer of a soluble vegfr-3 decoy receptor. Cancer Res. 2005;65:6901-6909

26. Lutgens E, Gorelik L, Daemen MJ, et al. Requirement for cd154 in the progression of atherosclerosis. Nat Med. 1999;5:1313-1316

27. Davies-Venn CA, Angermiller B, Wilganowski N, et al. Albumin-binding domain conjugate for near-infrared fluorescence lymphatic imaging. Mol Imaging Biol. 2012;14:301-314

28. Taub DD, Lloyd AR, Conlon $\mathrm{K}$, et al. Recombinant human interferon-inducible protein 10 is a chemoattractant for human monocytes and tymphocytes and promotes t cell adhesion to endothelial cells. J Exp Med. 1993;177:1809-1814

29. Forster R, Braun A, Worbs T. Lymph node homing of t cells and dendritic cells via afferent lymphatics. Trends Immunol. 2012;33:271-280

30. Kim H, Kataru RP, Koh GY. Regulation and implications of inflammatory lymphangiogenesis. Trends Immunol. 2012;33:350-356

31. $\mathrm{Xu} \mathrm{X}, \mathrm{Lu} \mathrm{H}$, Lin $\mathrm{H}$, et al. Lymphangiogenesis promotes inflammation and neointimal hyperplasia after adventitia removal in the rat carotid artery. Int $\mathrm{J}$ Cardiol. 2009;134:426-427

32. Barleon B, Sozzani S, Zhou D, et al. Migration of human monocytes in response to vascular endothelial growth factor (vegf) is mediated via the vegf receptor flt-1. Blood. 1996;87:3336-3343

33. Clauss $\mathrm{M}$, Weich $\mathrm{H}$, Breier $\mathrm{G}$, et al. The vascular endothelial growth factor receptor flt-1 mediates biological activities. Implications for a functional role of placenta growth factor in monocyte activation and chemotaxis. J Biol Chem. $1996 ; 271: 17629-17634$ 


\section{CHAPTER 7}

34. Collins PD, Connolly DT, Williams TJ. Characterization of the increase in vascular permeability induced by vascular permeability factor in vivo. $\mathrm{Br} \mathrm{J}$ Pharmacol. 1993;109:195-199

35. Grabner R, Lotzer K, Dopping $\mathrm{S}$, et al. Lymphotoxin beta receptor signaling promotes tertiary lymphoid organogenesis in the aorta adventitia of aged apoe-/- mice. J Exp Med. 2009;206:233-248

36. Issa A, Le TX, Shoushtari AN, et al. Vascular endothelial growth factor-c and cc chemokine receptor 7 in tumor cell-lymphatic cross-talk promote invasive phenotype. Cancer Res. 2009;69:349-357

37. Di Carlo E, D'Antuono T, Contento S, et al. Quilty effect has the features of lymphoid neogenesis and shares cxcl13-cxcr5 pathway with recurrent acute cardiac rejections. Am J Transplant. 2007;7:201-210

38. Zhuo W, Jia L, Song N, et al. The cxcl12-cxcr4 chemokine pathway: A novel axis regulates lymphangiogenesis. Clin Cancer Res. 2012;18:5387-5398

39. Zampell JC, Avraham T, Yoder N, et al. Lymphatic function is regulated by a coordinated expression of lymphangiogenic and anti-lymphangiogenic cytokines. Am J Physiol Cell Physiol. 2012;302:C392-404

40. Loetscher $\mathrm{M}$, Loetscher $\mathrm{P}$, Brass $\mathrm{N}$, et al. Lymphocyte-specific chemokine receptor cxcr3: Regulation, chemokine binding and gene localization. Eur J Immunol. 1998;28:3696-3705

41. Farber JM. Mig and ip-10: Cxc chemokines that target lymphocytes. J Leukoc Biol. 1997;61:246-257

42. Fraser R, Dubien L, Musil F, et al. Transport of cholesterol in thoracic duct lymph and serum of rhesus monkeys fed cholesterol with various food fats. Atherosclerosis. 1972;16:203-216

43. Planas-Paz L, Strilic B, Goedecke A, et al. Mechanoinduction of lymph vessel expansion. Embo J. 2012;31:788-804

44. Zernecke A, Bot I, Djalali-Talab Y, et al. Protective role of cxc receptor 4/cxc ligand 12 unveils the importance of neutrophils in atherosclerosis. Circ Res. 2008;102:209-217

45. Robertson AK, Hansson GK. T cells in atherogenesis: For better or for worse? Arterioscler Thromb Vasc Biol. 2006;26:2421-2432 
LYMPHANGIOGENESIS IN ATHEROSCLEROSIS: A PATH FOR T CELL TRAFFICKING? 


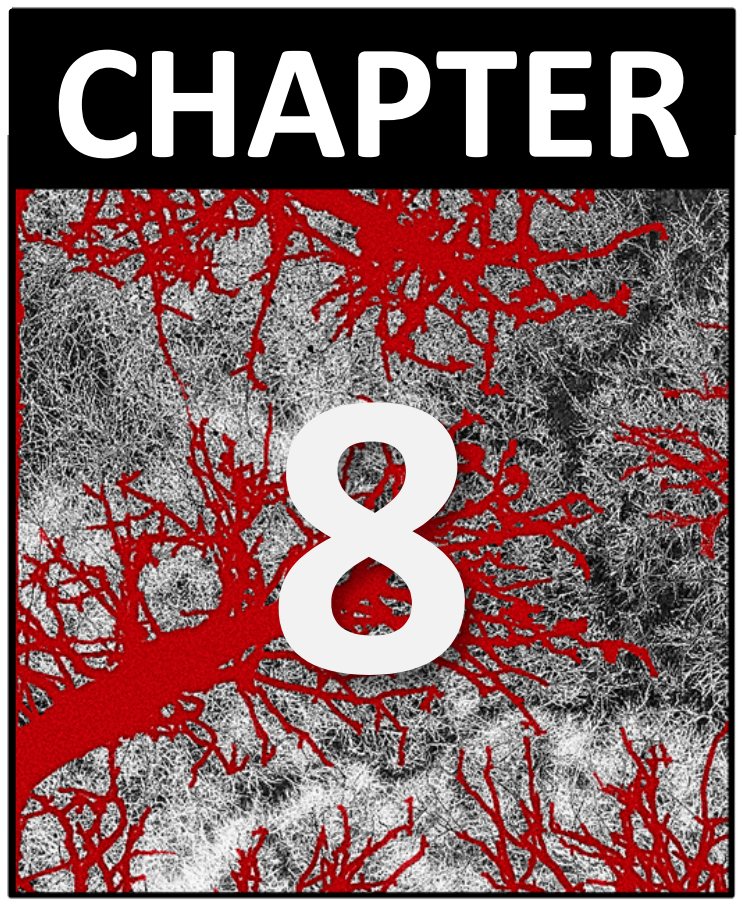




\section{General}

\section{discussion}


Atherosclerosis is at present one of the leading causes of mortality and morbidity in the world and is expected to remain so in the coming decades ${ }^{1,2}$. This fact alone clearly shows that a good understanding of disease development and progression is needed. One of the processes in lesion progression which has increasingly gained interest is the involvement of new vessel formation (neovascularization) in atherosclerosis. It has been hypothesized that newly formed plaque microvessels, similar to new vessels in tumors ${ }^{3}$, show an aberrant, dysfunctional phenotype ${ }^{4,5}$. Nevertheless, evidence showing a causal link between vascularization state and lesion progression in vivo has been lacking so far. Moreover, not only microvessels, but also lymphatic microvessels have recently been linked to atherosclerosis progression ${ }^{6,7}$, although their role therein has not yet been established.

The hypothesis underlying this thesis was that both angiogenesis and lymphangiogenesis are of key importance to atherosclerosis, and that the aberrant function of blood vessels and lymph vessels under inflammatory conditions promote atherosclerosis progression. Therefore, we aimed (I) to gain a better understanding of plaque neovascularization in vivo, (II) to assess whether specific targets for (lymph)angiogenesis in atherosclerotic lesions can be identified via a genomics-based approach, and (III) to gain more insights into the role of lymph vessel expansion in atherosclerosis. The main findings of this thesis are that:

1. Murine models of atherosclerosis exhibit adventitial neovessels and present a suitable model of human plaque neovascularization

2. Plaque-associated vasa vasorum in mice show a dysfunctional, proatherogenic characteristics

3. Aberrant expression and/or function of endothelial junction molecules is related to a leaky endothelial phenotype in murine atherosclerosis models

4. Genomics-based methods identify potential novel targets involved in human plaque angiogenesis

5. Plaque lymphangiogenesis, contrary to plaque angiogenesis, is beneficial for murine plaque development by reducing local inflammation 


\section{Murine models of atherosclerosis for studying plaque neovascularization}

A long-standing debate in literature has focused on the suitability of murine models for studying plaque neovascularization. This debate has been part of a broader discussions on the suitability of murine models of atherosclerosis in general, and has been reviewed extensively ${ }^{8-10}$. Generally, murine models for atherosclerosis can mimic human atherosclerotic lesion development to a large extent. Both the $\mathrm{ApoE}^{-/-}$and the $\mathrm{LDLr}^{-/-}$model are used very frequently and have been extensively characterized. Though the $\mathrm{ApoE}^{-1-}$ model is regarded as being slightly more inflammatory ${ }^{11}$, both models in many aspects resemble human atherosclerosis. Both models exhibit early and advanced lesions, and their cellular composition is very similar to human lesions. A major difference between the murine models and human lesion development, however, is the rare occurrence of plaque rupture and atherothrombosis in murine models, even after aggressive pharmacological, biomechanical or genetic interventions ${ }^{12-14}$. Recent data from Campbell et al suggests that differences in mechanical stress on the atherosclerotic lesion are an important reason for the lack of rupture in murine models of atherosclerosis ${ }^{15}$. In fact, they showed that in human atherosclerosis, biomechanical stresses are highest at the thinning cap, while in murine models these are highest in the media and adventitia distal from the atherosclerotic plaque.

In contrast, intraplaque hemorrhage (IPH) has been described more frequently in $\mathrm{ApoE}^{-/}$and $\mathrm{LDLr}^{-/}$, but is also present in several interventional models (Table 1) ${ }^{16-34}$. An important contributor to occurrence of IPH has been suggested to be the location of the atherosclerotic lesion, as Johnson described a location-specific prevalence of IPH in aged $\mathrm{ApoE}^{-/-}$mice, with the brachiocephalic artery showing the highest prevalence ${ }^{17}$. Indeed, Table 1 confirms this finding, although other models (collar placement, specific interventions) also reveal the occurrence of IPH on other locations throughout the arterial tree. Interestingly, although IPH is regarded to be a consequence of leaky plaque microvessels in human lesions, most of the studies describing IPH in murine models did not investigate plaque vascularization, were unable to detect plaque microvessels adequately, or were unable to conclusively link plaque neovascularization to IPH. This illustrates the need for studies which consequently investigate plaque neovascularization, its contribution in the atherosclerotic process, and its link to intraplaque hemorrhage. 


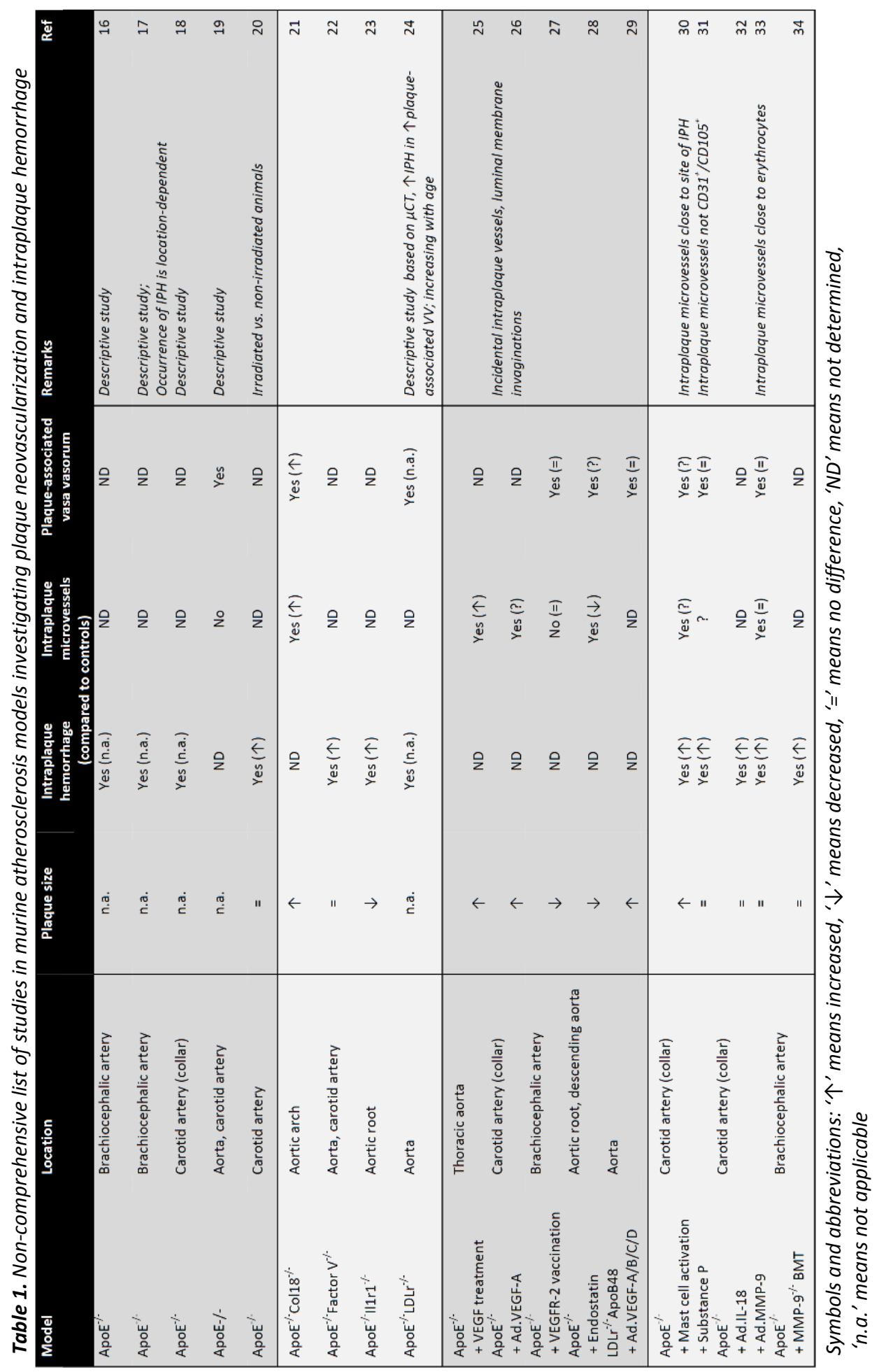


In the last few years, studies have been focusing more on determining the functional characteristics of plaque neovasculature, especially plaqueassociated vasa vasorum, with one of the most recent contribution being a study of Eriksson ${ }^{19}$. Not only did this study establish the presence of a plaquespecific vasa vasorum it was the first to establish increased adhesion of leukocytes to the vasa vasorum in vivo. This is in line with data from chapter 3 , where we showed a clear presence of a plaque-associated vasa vasorum in aged $\mathrm{ApoE}^{-/}$mice with advanced atherosclerotic lesion. These vasa vasorum showed distinct functional features compared to normal microvascular beds, e.g. reduced blood flow, increased permeability, leukocyte adhesion, and leukocyte transmigration. Moreover, the presence of plaque-associated vasa vasorum was also shown in atherosclerotic $\mathrm{LDLr}^{-/}$mice exhibiting advanced lesions after 40 weeks of diet (chapter $\mathbf{5}$ ). In summary, the studies in Table 1, as well as our studies showed that formation of plaque-specific vasa vasorum is a common event in murine models of atherosclerosis.

It however remains intriguing that plaque neovascularization in murine models seems to be limited to the adventitia, as the plaque-associated vasa vasorum did not traverse the medial layer, not in $\mathrm{ApoE}^{-/}$mice nor in $\mathrm{LDLr}^{-/-}$mice (chapters 3 and 5). This exposes an essential limitation of murine models, i.e. the general lack of intraplaque plaque angiogenesis in mice. In chapters $\mathbf{3}$ and $\mathbf{5}$ we were unable to not discern functional microvessels within the atherosclerotic lesions, and in chapter 3 we only showed occurrence of sprouts derived from the luminal endothelium. The latter brings up the issue of functionality of these sprouts and involvement in the atherosclerotic process. As Table 1 shows, the occurrence of intraplaque microvessels seems to be model-specific, and its link to intraplaque hemorrhage is weak. Several of these studies indicate the challenge of detecting intraplaque microvessels, which however does not necessarily mean that these microvessels are absent. Advances in staining techniques, as well as novel applications of imaging techniques as presented in this thesis (chapter 3) will aid in determining (functional characteristics of) intraplaque vessels in future studies.

Presently, the plaque-associated vasa vasorum can serve as a model for human plaque neovascularization. As shown by Langheinrich et al, there is a correlation between plaque vascularization and intraplaque hemorrhage ${ }^{24}$. Additionally, studying the plaque-associated vasa vasorum is able to give insights into functional properties of plaque microvessels, and allows 
assessment of the effect of interventions on plaque vascularization ${ }^{19}$. This will be aided by the use of other models which frequently show plaque vascularization, e.g. a collar-induced atherosclerosis model in the carotid artery of mice (Table 1).

\section{Causes and consequences of dysfunctional, leaky plaque microvasculature}

The angiogenic response occurring in human atherosclerosis is most likely a consequence of the hypoxic conditions present within the lesion. This is not only due to the increased distance from the lumen, exceeding the diffusion distance of oxygen, but also the increased oxygen demand in specific cell types, e.g. macrophages, under the inflammatory conditions. The latter aspect would also explain the presence of plaque hypoxia in murine lesions, even though the diffusion distance of oxygen probably is not limiting ${ }^{35}$. Although relieving tissue hypoxia in itself is suggested to be a beneficial consequence of plaque vascularization, the pathologic angiogenic response which is elicited, induces formation of dysfunctional microvessels. This dysfunctional phenotype can have a myriad of causes, one of which is aberrations of the endothelial junctions.

In chapters $\mathbf{4}$ and $\mathbf{5}$ we examined two junctional molecules, and their role in atherosclerosis. On the one hand, JAM-A is an endothelial junction molecule which previously was associated with leukocyte adhesion ${ }^{36}$, but which is also expressed on other cell types and has various cell-type specific functions. By looking specifically to macrovascular endothelial JAM-A in the (atherosclerotic) carotid artery, we elucidated its role in leukocyte adhesion and transmigration, but not in vascular permeability. Under atherosclerotic conditions or disturbed flow, macrovascular JAM-A is redistributed, leading to enhanced leukocyte adhesion and transmigration. On the other hand, VEcadherin, another endothelial junction molecule, is mostly involved in leukocyte transmigration and vascular permeability, not leukocyte adhesion ${ }^{37}$. Importantly, on plaque-associated vasa vasorum, blocking of VE-cadherin did not result in additional leukocyte transmigration or vascular permeability, revealing a potential defect of VE-cadherin function in these microvessels, or a lack of involvement of VE-cadherin in plaque-associated vasa vasorum.

These two junctional molecules are good illustrations of the complexity of the endothelial barrier, in which one molecule (JAM-A) is upregulated in the microvasculature under atherosclerotic conditions and elicits pro-atherogenic 
properties, while another molecule (VE-cadherin) potentially is dysfunctional and may cause pro-atherogenic properties. Concomitantly, to achieve a comprehensive view of the endothelial barrier in plaque microvessels, it would be of great interest to investigate the function of other endothelial junction molecules, e.g. claudins or occludins ${ }^{38}$ in plaque-associated vasa vasorum. Alternatively, it would be interesting to assess which process is most prevalently contributing to plaque progression, i.e. leukocyte adhesion and transmigration (leukocyte recruitment), or general microvascular permeability. More insights into these mechanisms, their relative contributions, and the most important molecular mediators (specific junctional molecules) may then help guide the search for specific therapeutic targets.

It should be noted that micro- and even macrovascular function is not solely determined by the integrity of endothelial junctions (Figure 1). As we showed in chapter 3, ultrastructural analysis of plaque-associated vasa vasorum did not reveal any gross structural defects in the endothelial junctions, yet microvascular permeability was increased. This might be linked to alterations in the endothelial glycocalyx distribution. Changes in glycocalyx composition, distribution or thickness are already linked to several diseases, most importantly type II diabetes ${ }^{39-41}$, and show changes in vascular permeability (Figure 1). As such, the glycocalyx should not be neglected when studying microvascular function in plaque neovascularization.

Additionally, pericytes, which surround blood vessels, and are important for vessel maturation and stability, are involved in (micro)vascular function. Ablation of pericytes has been shown to increase vascular permeability (Figure 1) ${ }^{42-44}$. Incomplete or absent pericyte coverage has been shown in plaque microvasculature in human atherosclerotic lesions (chapter 6) and may thus contribute to the leaky phenotype, although we did not observe any difference in pericyte coverage between stable and ruptured lesions. Pericyte coverage has not been addressed in our experimental animal studies in this thesis, but the effect of aberrant or absent pericyte coverage should be studied functionally in vivo to assess pericyte contribution to microvascular permeability. A useful model for such studies would be transgenic mice expressing platelet-derived growth factor beta (PDGF- $\beta$ ), a growth factor involved in the recruitment and function of pericytes, lacking the proteoglycan binding domain (PDGF- $\beta^{\text {ret/ret }}$ ) resulting in a partial loss of function ${ }^{45}$. 


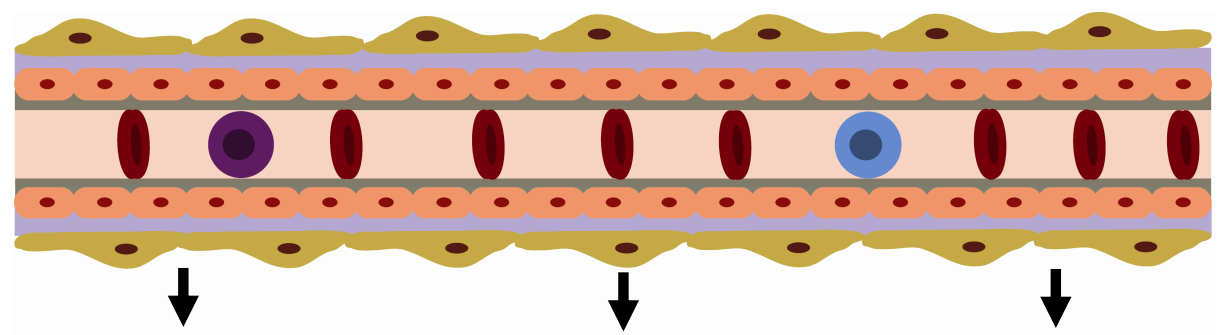

EC junction loosening

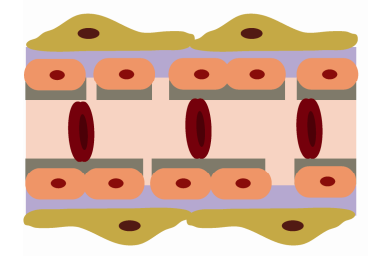

Glycocalyx aberrations
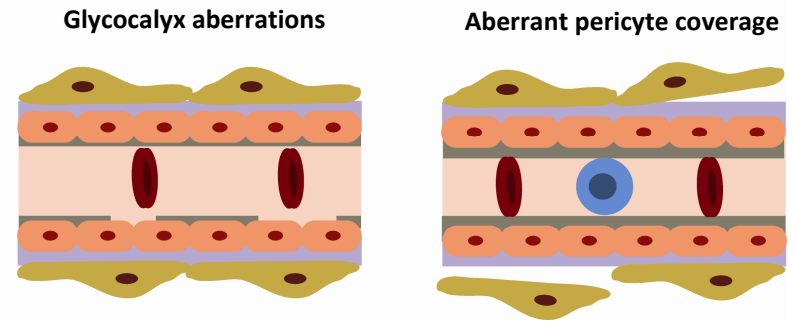
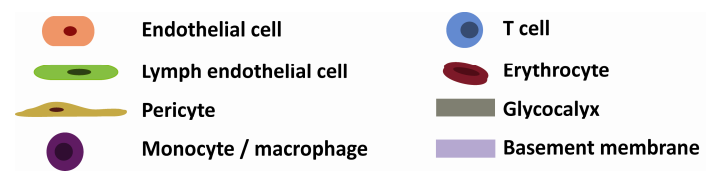

Figure 1. Schematic representation of mechanisms involved in vascular permeability. Under physiological conditions (upper panel), mature microvessels consist of a continuous endothelial layer, with stable endothelial barrier function. Glycocalyx is expressed on the endothelium and forms a barrier between the cells in the blood and the endothelial surface. Abluminally, the microvessel is covered by pericytes. Under inflammatory conditions or pathological angiogenesis, several processes can cause increased vascular permeability. The first process (bottom left panel) is disturbed endothelial barrier function, e.g. by loosening of the endothelial junctions. The second process (bottom middle panel) is aberrations in glycocalyx thickness, composition or distribution. A third process (bottom right panel) is abnormal or absent pericyte coverage leading to an immature vessel phenotype. In reality, a combination of the mechanisms mentioned in this figure will determine vascular permeability in vivo.

\section{Novel players of pathologic (lymph)angiogenesis in atherosclerosis}

Clearly, the variety of contributors to (micro)vascular function, and the myriad of potential molecular mediators thereof, make it difficult to dissect the regulatory pathways that are exclusively involved in plaque (lymph)angiogenesis, or eventually even determine potential targets for imaging or intervention, as discussed later. In chapter 6, we therefore applied a genomics-based approach to identify genes involved in plaque (lymph)angiogenesis. By correlating microarray analysis data with defined phenotypical traits, we established gene modules which correlated with plaque vascularization. From this, we could select highly correlating genes, as 
well as a five miRNAs which may be involved in regulation of plaque neovascularization. Moreover, these genes and miRNAs were predicted to have a central role in the gene module associated with plaque angiogenesis.

The next step would be to validate the genes and miRNAs for expression in endothelial cells, and establish their function in vitro and in vivo. In vitro tests could establish a role of the genes and miRNAs in endothelial cell proliferation, migration, tube formation capacity or endothelial barrier function. From there, genes of interest could be studied in vivo, first in angiogenic responses in vivo in e.g. a zebrafish model ${ }^{46,47}$, and later with a focus on microvascular function in atherosclerosis, following the methods described in chapters 3-5. Such an approach will reveal insights into contributions of these genes in (pathological) plaque angiogenesis.

An additional goal could be determining novel targets which could serve as a molecular marker for detection of patients at risk of plaque rupture, or which could be used for targeted intervention. The development of molecular markers for non-invasive imaging (e.g. magnetic resonance imaging (MRI) or positron emission tomography (PET) ${ }^{48-51}$ ) based on plaque angiogenesis markers, e.g. $\alpha_{v} \beta_{3}$-integrin, has been studied in animal models over the last years, and has shown promising results ${ }^{52-54}$. First clinical trials are ongoing, but depending on their outcome, development of new markers for (lymph)angiogenesis may be required.

Aside from molecular markers for detection of plaque (lymph)angiogenesis, targeted therapy could be a further goal. At present, no targeted interventions focused on plaque (lymph)angiogenesis have been described in human atherosclerosis. Use of statins has been correlated with decreased plaque vascularization ${ }^{55,56}$, although the pleitropic effects of statins make it nearly impossible do discern if it concerns a direct effect on angiogenesis, or whether it is attributable to the overall beneficial properties of statins on atherosclerosis ${ }^{57}$. Targeted anti-angiogenic interventions in humans have been conducted especially in cancer therapy (e.g. VEGFdepleting antibodies or VEGF-based gene therapy) reviewed in 58-60, but clinical studies for anti-angiogenic interventions in atherosclerosis are virtually lacking. Only one study investigated the effect of bevacizumab-eluting stents in restenosis ${ }^{61}$, and showed encouraging results, with reduced neointimal hyperplasia after stenting. Except for this study and several ongoing clinical trials, however, anti-angiogenic therapy in atherosclerosis is absent. 
The lack of studies investigating anti-angiogenic therapy in human atherosclerosis will in part due to the fact that angiogenesis is an essential process in physiology, e.g. growth and wound healing. Therefore, angiogenesis cannot be simply inhibited over long periods of time, without causing serious deleterious effects. Local delivery of anti-angiogenic might be an option, although this would require procedures like stenting ${ }^{61}$. Identifying potential targets is complicated by the great molecular overlap between angiogenesis and lymphangiogenesis, and between angiogenesis and neurogenesis ${ }^{62,63}$. Evidently, intervention should not interfere with neurogenesis, physiological angiogenesis or lymphangiogenesis. An optimal therapeutic target for plaque angiogenesis would moreover not be directed against inhibition of the angiogenic process itself, but ideally should be directed towards normalizing microvascular function, preventing microvascular leakage and increased leukocyte recruitment. Vessel normalization has previously been described to be effective in treatment of e.g. tumors ${ }^{64}$ or hereditary hemorrhagic telangiectasia ${ }^{65}$, but currently involve non-targeted treatment. Genomicsbased approaches can aid in determining novel targets, taking into account their involvement in a certain phenotype and their connections to various pathways.

\section{Lymphangiogenesis is beneficial in atherosclerosis progression by reducing local inflammation}

The role of the lymphatic system in atherosclerosis, and possible options for targeted intervention, also requires extensive study. In chapter 7 , we showed that lymphangiogenesis acts beneficial in atherosclerosis, by reducing local T cell content. This role of lymphangiogenesis is in sharp contrast to that of the angiogenic response. Since leukocyte recruitment is increased in atherosclerosis, and lymph vessels are able to remove cells from the atherosclerotic lesion, stimulating lymph vessel expansion may be a novel approach to alleviate atherosclerosis progression. Such an approach may be regarded counterintuitive at first, since lymphangiogenesis has been shown not to be beneficial at all in other diseases. Indeed, in tumorigenesis, lymphangiogenesis is upregulated, but is an important contributor to tumor metastasis 66, 67. Also in e.g. inflammatory bowel disease, the role of lymphangiogenesis is not clear, but has been regarded as being detrimental ${ }^{68}$. Nevertheless, initial studies in mice have described pro-lymphangiogenic 
therapy for instance in relieving edema formation ${ }^{69}$, but may also be beneficial in atherosclerosis. Future studies however need to more firmly establish the role of lymph vessels in atherosclerosis.

Interestingly, lymph vessel density (D2-40 area) in human lesions showed a clear segregation between stable and ruptured segments (chapter 6). As such, lymph vessel expansion in atherosclerotic lesions may present a promising molecular marker for screening of patients at risk for plaque rupture, and should be investigated in more detail.

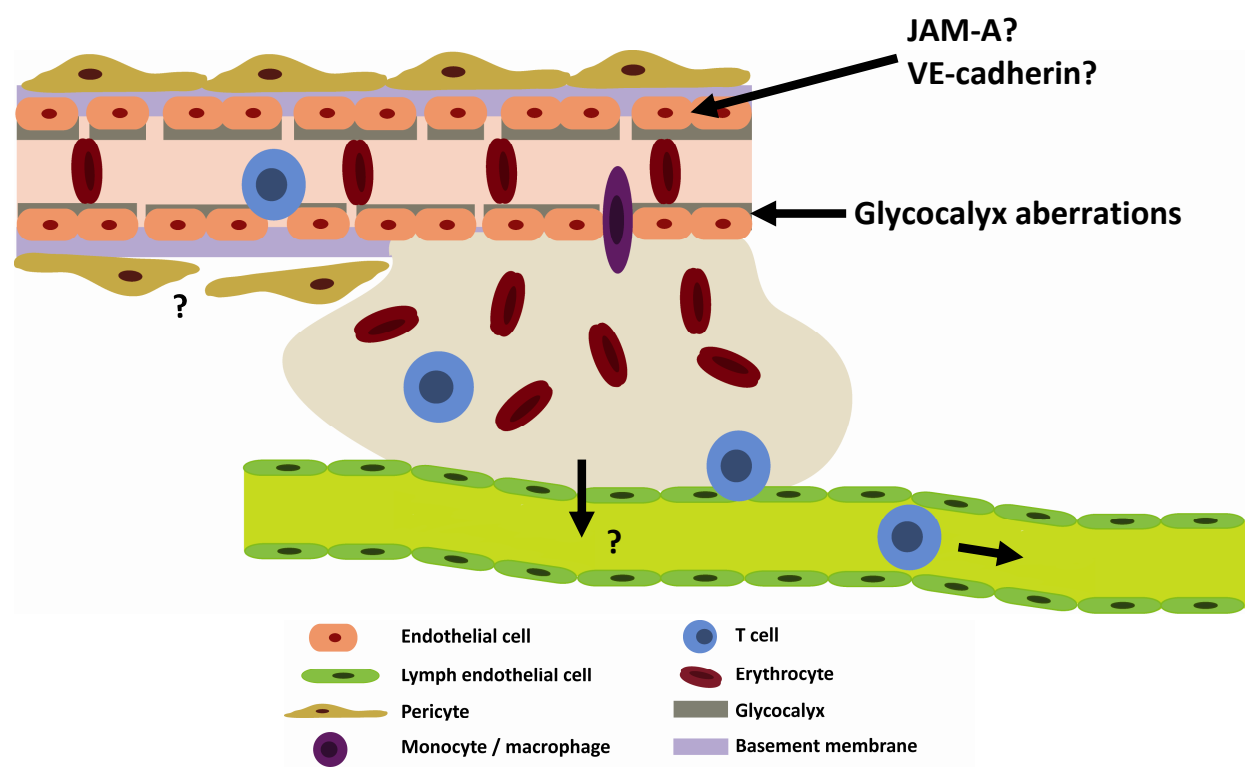

Figure 2. Schematic representation of adventitial plaque microvessels and lymph vessels function in murine atherosclerosis. This thesis established that plaque-associated vasa vasorum in mice showed a dysfunctional, pro-atherogenic phenotype, characterized by increased microvascular permeability and enhanced leukocyte adhesion and transmigration. This proatherogenic phenotype may in part be due to aberrant glycocalyx distribution, or altered expression/function of the endothelial barrier. Alternatively, although not studied in this thesis, absence of pericytes may also contribute to the increase in permeability. Adventitial lymph vessels are beneficial for plaque development by reducing local $T$ cell content. In addition, although not studied in this thesis, adventitial lymph vessels may drain the, potentially cytokineand chemokine-rich, fluid exudate from the adventitia. 


\section{CHAPTER 8}

\section{Concluding remarks and future perspectives}

In this thesis we addressed the role of angiogenesis and lymphangiogenesis in atherosclerosis. We confirmed a pro-atherogenic role of plaque angiogenesis, with a dysfunctional phenotype of plaque microvessels in vivo (Figure 2). This phenotype (in part) may be due to the altered distribution and function of endothelial junction molecules like JAM-A and VE-cadherin (Figure 2). In contrast, lymphangiogenesis showed a protective effect in atherosclerosis by draining inflammatory cells from the lesion (Figure 2).

The pathological angiogenic and lymphangiogenic response are however not yet completely understood. To further unravel the regulatory pathways involved herein, we used a genomics-based approach, by which we identified genes and regulatory miRNAs involved in plaque angiogenesis. After in vitro and in vivo validation, this approach will shed new light into the regulation of plaque (lymph)angiogenesis, and could in future potentially aid in establishing markers for molecular imaging or targets for interventions in atherosclerosis.

Future studies will need to establish a comprehensive model of plaque angiogenesis and lymphangiogenesis, and the contribution of lesional and adventitial microvessels and lymph vessels to atherosclerosis. 


\section{References}

1. Roger VL, Go AS, Lloyd-Jones DM, et al. Heart disease and stroke statistics-2011 update: A report from the american heart association. Circulation. 2011;123:e18-e209

2. Sanz J, Moreno PR, Fuster V. The year in atherothrombosis. J Am Coll Cardiol. 2012;60:932-942

3. De Bock K, Cauwenberghs S, Carmeliet P. Vessel abnormalization: Another hallmark of cancer? Molecular mechanisms and therapeutic implications. Curr Opin Genet Dev. 2011;21:73-79

4. Sanz J, Fayad ZA. Imaging of atherosclerotic cardiovascular disease. Nature. 2008;451:953-957

5. Sluimer JC, Daemen MJ. Novel concepts in atherogenesis: Angiogenesis and hypoxia in atherosclerosis. J Pathol. 2009;218:7-29

6. Drozdz K, Janczak D, Dziegiel $\mathrm{P}$, et al. Adventitial lymphatics and atherosclerosis. Lymphology. 2012;45:26-33

7. Kholova I, Dragneva G, Cermakova P, et al. Lymphatic vasculature is increased in heart valves, ischaemic and inflamed hearts and in cholesterol-rich and calcified atherosclerotic lesions. Eur J Clin Invest. 2011;41:487-497

8. Jackson $\mathrm{CL}$, Bennett $\mathrm{MR}$, Biessen $\mathrm{EA}$, et al. Assessment of unstable atherosclerosis in mice. Arterioscler Thromb Vasc Biol. 2007;27:714-720

9. Schwartz SM, Galis ZS, Rosenfeld ME, et al. Plaque rupture in humans and mice. Arterioscler Thromb Vasc Biol. 2007;27:705-713

10. Bentzon JF, Falk E. Atherosclerotic lesions in mouse and man: Is it the same disease? Curr Opin Lipidol. 2010;21:434-440

11. Zadelaar S, Kleemann R, Verschuren L, et al. Mouse models for atherosclerosis and pharmaceutical modifiers. Arterioscler Thromb Vasc Biol. 2007;27:17061721

12. Clarke $M$, Bennett $M$. The emerging role of vascular smooth muscle cell apoptosis in atherosclerosis and plaque stability. American journal of nephrology. 2006;26:531-535

13. Kuijpers MJ, Gilio K, Reitsma S, et al. Complementary roles of platelets and coagulation in thrombus formation on plaques acutely ruptured by targeted ultrasound treatment: A novel intravital model. Journal of thrombosis and haemostasis : JTH. 2009;7:152-161

14. von der Thusen JH, van Vlijmen BJ, Hoeben RC, et al. Induction of atherosclerotic plaque rupture in apolipoprotein e-/- mice after adenovirusmediated transfer of p53. Circulation. 2002;105:2064-2070

15. Campbell IC, Weiss D, Suever JD, et al. Biomechanical modeling and morphology analysis indicates plaque rupture due to mechanical failure unlikely in atherosclerosis-prone mice. American journal of physiology. Heart and circulatory physiology. 2012

16. Rosenfeld ME, Polinsky P, Virmani R, et al. Advanced atherosclerotic lesions in the innominate artery of the apoe knockout mouse. Arterioscler Thromb Vasc Biol. 2000;20:2587-2592 
17. Johnson JL, Jackson CL. Atherosclerotic plaque rupture in the apolipoprotein e knockout mouse. Atherosclerosis. 2001;154:399-406

18. Sasaki T, Kuzuya M, Nakamura K, et al. A simple method of plaque rupture induction in apolipoprotein e-deficient mice. Arterioscler Thromb Vasc Biol. 2006;26:1304-1309

19. Eriksson EE. Intravital microscopy on atherosclerosis in apolipoprotein edeficient mice establishes microvessels as major entry pathways for leukocytes to advanced lesions. Circulation. 2011;124:2129-2138

20. Stewart FA, Heeneman S, Te Poele J, et al. Ionizing radiation accelerates the development of atherosclerotic lesions in apoe-/- mice and predisposes to an inflammatory plaque phenotype prone to hemorrhage. The American journal of pathology. 2006;168:649-658

21. Moulton KS, Olsen BR, Sonn S, et al. Loss of collagen xviii enhances neovascularization and vascular permeability in atherosclerosis. Circulation. 2004;110:1330-1336

22. Eitzman DT, Westrick RJ, Shen Y, et al. Homozygosity for factor v leiden leads to enhanced thrombosis and atherosclerosis in mice. Circulation. 2005;111:1822-1825

23. Alexander MR, Moehle CW, Johnson JL, et al. Genetic inactivation of il-1 signaling enhances atherosclerotic plaque instability and reduces outward vessel remodeling in advanced atherosclerosis in mice. The Journal of clinical investigation. 2012;122:70-79

24. Langheinrich AC, Michniewicz A, Sedding DG, et al. Correlation of vasa vasorum neovascularization and plaque progression in aortas of apolipoprotein e(-/-)/low-density lipoprotein(-/-) double knockout mice. Arterioscler Thromb Vasc Biol. 2006;26:347-352

25. Celletti FL, Waugh JM, Amabile PG, et al. Vascular endothelial growth factor enhances atherosclerotic plaque progression. Nature medicine. 2001;7:425429

26. Lucerna $M$, Zernecke $A$, de Nooijer $\mathrm{R}$, et al. Vascular endothelial growth factor-a induces plaque expansion in apoe knock-out mice by promoting de novo leukocyte recruitment. Blood. 2007;109:122-129

27. Hauer $A D$, van Puijvelde $G H$, Peterse $N$, et al. Vaccination against vegfr2 attenuates initiation and progression of atherosclerosis. Arterioscler Thromb Vasc Biol. 2007;27:2050-2057

28. Moulton KS, Heller E, Konerding MA, et al. Angiogenesis inhibitors endostatin or tnp-470 reduce intimal neovascularization and plaque growth in apolipoprotein e-deficient mice. Circulation. 1999;99:1726-1732

29. Leppanen P, Koota S, Kholova I, et al. Gene transfers of vascular endothelial growth factor-a, vascular endothelial growth factor-b, vascular endothelial growth factor-c, and vascular endothelial growth factor-d have no effects on atherosclerosis in hypercholesterolemic low-density lipoproteinreceptor/apolipoprotein b48-deficient mice. Circulation. 2005;112:1347-1352

30. Bot I, de Jager SC, Zernecke A, et al. Perivascular mast cells promote atherogenesis and induce plaque destabilization in apolipoprotein e-deficient mice. Circulation. 2007;115:2516-2525 
31. Bot I, de Jager SC, Bot $\mathrm{M}$, et al. The neuropeptide substance $\mathrm{p}$ mediates adventitial mast cell activation and induces intraplaque hemorrhage in advanced atherosclerosis. Circ Res. 2010;106:89-92

32. de Nooijer R, von der Thusen JH, Verkleij $\mathrm{CJ}$, et al. Overexpression of il-18 decreases intimal collagen content and promotes a vulnerable plaque phenotype in apolipoprotein-e-deficient mice. Arterioscler Thromb Vasc Biol. 2004;24:2313-2319

33. de Nooijer R, Verkleij CJ, von der Thusen JH, et al. Lesional overexpression of matrix metalloproteinase- 9 promotes intraplaque hemorrhage in advanced lesions but not at earlier stages of atherogenesis. Arterioscler Thromb Vasc Biol. 2006;26:340-346

34. Gough PJ, Gomez IG, Wille PT, et al. Macrophage expression of active mmp-9 induces acute plaque disruption in apoe-deficient mice. The Journal of clinical investigation. 2006;116:59-69

35. Torres Filho IP, Leunig M, Yuan F, et al. Noninvasive measurement of microvascular and interstitial oxygen profiles in a human tumor in scid mice. Proceedings of the National Academy of Sciences of the United States of America. 1994;91:2081-2085

36. Martin-Padura I, Lostaglio S, Schneemann $\mathrm{M}$, et al. Junctional adhesion molecule, a novel member of the immunoglobulin superfamily that distributes at intercellular junctions and modulates monocyte transmigration. The Journal of cell biology. 1998;142:117-127

37. Vestweber D. Ve-cadherin: The major endothelial adhesion molecule controlling cellular junctions and blood vessel formation. Arterioscler Thromb Vasc Biol. 2008;28:223-232

38. Bazzoni G. Endothelial tight junctions: Permeable barriers of the vessel wall. Thrombosis and haemostasis. 2006;95:36-42

39. Perrin RM, Harper SJ, Bates DO. A role for the endothelial glycocalyx in regulating microvascular permeability in diabetes mellitus. Cell biochemistry and biophysics. 2007; 49:65-72

40. Salmon AH, Satchell SC. Endothelial glycocalyx dysfunction in disease: Albuminuria and increased microvascular permeability. I Pathol. 2012;226:562-574

41. Reitsma S, Slaaf DW, Vink $\mathrm{H}$, et al. The endothelial glycocalyx: Composition, functions, and visualization. Pflugers Archiv : European journal of physiology. 2007;454:345-359

42. Bjarnegard M, Enge M, Norlin J, et al. Endothelium-specific ablation of pdgfb leads to pericyte loss and glomerular, cardiac and placental abnormalities. Development. 2004;131:1847-1857

43. Cao R, Xue Y, Hedlund EM, et al. Vegfr1-mediated pericyte ablation links vegf and plgf to cancer-associated retinopathy. Proceedings of the National Academy of Sciences of the United States of America. 2010;107:856-861

44. Huang FJ, You WK, Bonaldo $\mathrm{P}$, et al. Pericyte deficiencies lead to aberrant tumor vascularizaton in the brain of the ng2 null mouse. Developmental biology. 2010;344:1035-1046 
45. Raines SM, Richards OC, Schneider LR, et al. Loss of pdgf-b activity increases hepatic vascular permeability and enhances insulin sensitivity. American journal of physiology. Endocrinology and metabolism. 2011;301:E517-526

46. Bos FL, Caunt M, Peterson-Maduro J, et al. Ccbe1 is essential for mammalian lymphatic vascular development and enhances the lymphangiogenic effect of vascular endothelial growth factor-c in vivo. Circ Res. 2011;109:486-491

47. Habeck H, Odenthal J, Walderich B, et al. Analysis of a zebrafish vegf receptor mutant reveals specific disruption of angiogenesis. Curr Biol. 2002;12:14051412

48. Corti R, Fuster V. Imaging of atherosclerosis: Magnetic resonance imaging. European heart journal. 2011;32:1709-1719b

49. Degnan AJ, Young VE, Gillard JH. Advances in noninvasive imaging for evaluating clinical risk and guiding therapy in carotid atherosclerosis. Expert review of cardiovascular therapy. 2012;10:37-53

50. Dobrucki LW, Sinusas AJ. Pet and spect in cardiovascular molecular imaging. Nature reviews. Cardiology. 2010;7:38-47

51. Mulder WJ, Strijkers GJ, Vucic E, et al. Magnetic resonance molecular imaging contrast agents and their application in atherosclerosis. Topics in magnetic resonance imaging : TMRI. 2007;18:409-417

52. Burtea C, Laurent S, Murariu O, et al. Molecular imaging of alpha $v$ beta3 integrin expression in atherosclerotic plaques with a mimetic of rgd peptide grafted to gd-dtpa. Cardiovascular research. 2008;78:148-157

53. Laitinen I, Saraste A, Weidl E, et al. Evaluation of alphavbeta3 integrintargeted positron emission tomography tracer 18f-galacto-rgd for imaging of vascular inflammation in atherosclerotic mice. Circulation. Cardiovascular imaging. 2009;2:331-338

54. Winter PM, Morawski AM, Caruthers SD, et al. Molecular imaging of angiogenesis in early-stage atherosclerosis with alpha(v)beta3-integrintargeted nanoparticles. Circulation. 2003;108:2270-2274

55. Bot I, Jukema JW, Lankhuizen IM, et al. Atorvastatin inhibits plaque development and adventitial neovascularization in apoe deficient mice independent of plasma cholesterol levels. Atherosclerosis. 2011;214:295-300

56. Koutouzis M, Nomikos A, Nikolidakis S, et al. Statin treated patients have reduced intraplaque angiogenesis in carotid endarterectomy specimens. Atherosclerosis. 2007;192:457-463

57. Karthikeyan VJ, Lip GY. Statins and intra-plaque angiogenesis in carotid artery disease. Atherosclerosis. 2007;192:455-456

58. Petrillo M, Scambia G, Ferrandina G. Novel targets for vegf-independent antiangiogenic drugs. Expert opinion on investigational drugs. 2012;21:451-472

59. Sitohy B, Nagy JA, Dvorak HF. Anti-vegf/vegfr therapy for cancer: Reassessing the target. Cancer research. 2012;72:1909-1914

60. Vuorio T, Jauhiainen S, Yla-Herttuala S. Pro- and anti-angiogenic therapy and atherosclerosis with special emphasis on vascular endothelial growth factors. Expert opinion on biological therapy. 2012;12:79-92

61. Stefanadis C, Toutouzas K, Tsiamis E, et al. First-in-man study with bevacizumab-eluting stent: A new approach for the inhibition of atheromatic 
plaque neovascularisation. Eurolntervention : journal of EuroPCR in collaboration with the Working Group on Interventional Cardiology of the European Society of Cardiology. 2008;3:460-464

62. Carmeliet P. Blood vessels and nerves: Common signals, pathways and diseases. Nat Rev Genet. 2003;4:710-720

63. Eichmann A, Le Noble F, Autiero $M$, et al. Guidance of vascular and neural network formation. Curr Opin Neurobiol. 2005;15:108-115

64. Batchelor TT, Sorensen AG, di Tomaso E, et al. Azd2171, a pan-vegf receptor tyrosine kinase inhibitor, normalizes tumor vasculature and alleviates edema in glioblastoma patients. Cancer cell. 2007;11:83-95

65. Lebrin F, Srun S, Raymond K, et al. Thalidomide stimulates vessel maturation and reduces epistaxis in individuals with hereditary hemorrhagic telangiectasia. Nature medicine. 2010;16:420-428

66. Jain RK, Padera TP. Prevention and treatment of lymphatic metastasis by antilymphangiogenic therapy. Journal of the National Cancer Institute. 2002;94:785-787

67. Sleeman JP, Thiele W. Tumor metastasis and the lymphatic vasculature. International journal of cancer. Journal international du cancer. 2009;125:2747-2756

68. Alexander JS, Chaitanya GV, Grisham MB, et al. Emerging roles of lymphatics in inflammatory bowel disease. Annals of the New York Academy of Sciences. 2010;1207 Suppl 1:E75-85

69. Holopainen T, Bry M, Alitalo K, et al. Perspectives on lymphangiogenesis and angiogenesis in cancer. Journal of surgical oncology. 2011;103:484-488 


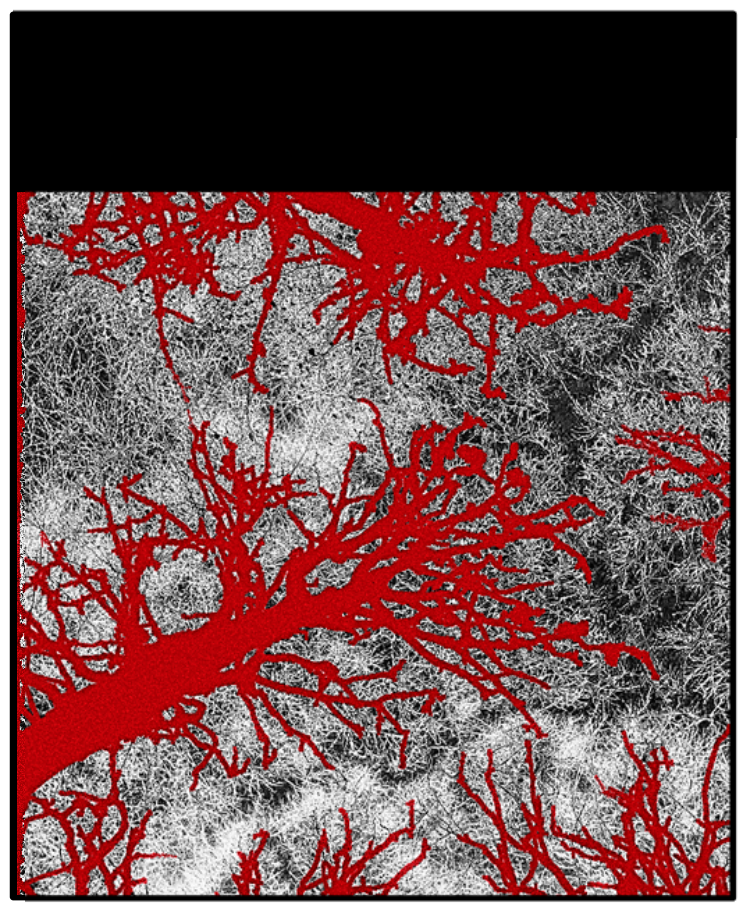




\section{Summary}


Cardiovascular diseases are the leading cause of mortality worldwide, accounting for about 17 million deaths globally every year. The most prevalent cardiovascular diseases are acute myocardial infarction and stroke, which are mostly caused by occlusion of arteries as a result of rupture of advanced atherosclerotic plaques.

Atherosclerosis is a progressive, chronic inflammatory disease, characterized by accumulation of lipids, and cellular and fibrous constituents in the arterial tree at sites of disturbed flow. At these sites, LDL cholesterol enters the vessel wall, is modified, and elicits recruitment of monocytes to the vessel wall. These monocytes differentiate into macrophages, which take up the oxidized LDL, develop into foam cells, are rendered immobile, and as a result accumulate in the vessel wall. This causes a persistent inflammatory response in the vessel wall and, with time, the development of advanced atherosclerotic lesions with a necrotic and lipid-rich core which is covered by a fibrous cap. Production of several types of proteases, as well as cell death, and erosion will destabilize the fibrous cap, allowing the plaque to evolve to a rupture-prone plaque phenotype. Upon plaque rupture, the pro-thrombotic constituents of the necrotic core will come into contact with the blood, thus causing thrombus formation, and potentially causing acute myocardial infarction or stroke.

Angiogenesis, the development of new blood vessels, is also an important factor in atherosclerosis. In human atherosclerotic lesions, there is a clear association between expansion of microvessels and plaque progression, both in the adventitia and the intima. Plaque microvasculature has been attributed both beneficial and detrimental contributions to atherosclerosis progression. On the one hand, plaque microvasculature has been suggested to be a means of efflux of lipids and inflammatory cells. On the other hand, plaque microvessels have been linked to influx of lipids and inflammatory cells, and show a clear association with a more instable plaque phenotype. Yet, the exact role of plaque microvasculature in vivo is not completely understood. Recently, also lymph vessels have been implicated in atherosclerosis development, and were shown to be increased in later stages of plaque progression. The role of plaque lymph vessels, however, is still elusive.

Therefore, the hypothesis of this thesis is that both angiogenesis and lymphangiogenesis are of key importance to atherosclerosis, and that the aberrant function of blood vessels and lymph vessels under inflammatory conditions promote atherosclerosis progression. 
Our first studies (chapters 2-5) were focused on establishing functional characteristics of atherosclerotic plaque microvasculature using an in vivo multiphoton laser scanning microscopy (MPLSM) approach. Chapter 2 reviewed the current state of in vivo cardiovascular imaging using MPLSM, and discussed the benefits and shortcomings of the technique.

In chapter 3, plaque microvasculature was studied in an $\mathrm{ApoE}^{-/}$mouse model, where it was shown that plaque neovascularization was present in the outer layer of atherosclerotic blood vessels. Furthermore, it was found that only aged $\mathrm{ApoE}^{-/-}$mice showed these microvessels, and that these were not present in non-atherosclerotic $\mathrm{C} 57 \mathrm{BI} / 6$ mice or young atherosclerotic $\mathrm{ApoE}^{-/-}$ mice. It was also shown that the adventitial microvessels exhibited active angiogenesis, low blood flow, increased permeability, and leukocyte adhesion and extravasation. These functional features can generally be considered proatherogenic features.

In order to explore possible causes of this leaky phenotype, we studied the function of two important endothelial junction molecules, JAM-A and VEcadherin. In chapter 4 , the role of the adhesion molecule JAM-A on endothelial permeability and leukocyte adhesion was investigated. It was shown that in arteries, JAM-A was important for adhesion and transmigration of leukocytes, and that alteration in the distribution of endothelial JAM-A induced increased leukocyte recruitment, while there was no effect on vascular permeability. Another endothelial junction molecule involved in endothelial barrier function, VE-cadherin, was studied in chapter 5 . In this study, the effect of acute deterioration of the endothelial barrier function in plaque microvessels was examined. We could show that blocking VE-cadherin dimerization induced an increase in microvascular permeability and leukocyte transmigration in control microvessels. These parameters were however unchanged in plaque microvessels after VE-cadherin blocking. These findings suggested that either VE-cadherin may already be dysfunctional in adventitial microvessels and hence cannot be disrupted additionally, or alternatively are not involved in microvascular permeability in adventitial microvessels at all.

In a subsequent study in chapter 6 , we sought to identify novel genetic targets involved in plaque angiogenesis and lymphangiogenesis using a genomics-based study in human atherosclerotic lesions samples. To this end, we correlated microarray data from stable and ruptured atherosclerotic plaque segments to microvessel and lymph vessel density. A set of sixteen genes and 
five miRNAs were linked to plaque angiogenesis, while initial analyses were unable to uncover novel genes associated with plaque lymphangiogenesis.

With the role of lymphangiogenesis being poorly understood, we performed several intervention studies in atherosclerotic $\mathrm{ApoE}^{-/-}$mice to gain a better understanding of the role of the lymphatic system in atherosclerosis. In chapter 7, we showed that removal of the lymph node draining the (atherosclerotic) carotid artery had an adverse effect on plaque progression, resulting in increased plaque size and induction of a more inflammatory phenotype with significantly increased $T$ cell numbers. Similarly, systemic inhibition of lymphangiogenesis induced an enhanced $T$ cell content in atherosclerotic lesions. Taken together, these data suggested a beneficial role of adventitial lymph vessels during plaque progression.

In chapter 8, the results described in this thesis and potential implications hereof were discussed and put in perspective. Also, future directions were given for further study of angiogenesis and lymphangiogenesis in atherosclerosis. In conclusion, this thesis provides new insights into the contribution of plaque angiogenesis and lymphangiogenesis to atherosclerosis progression. 


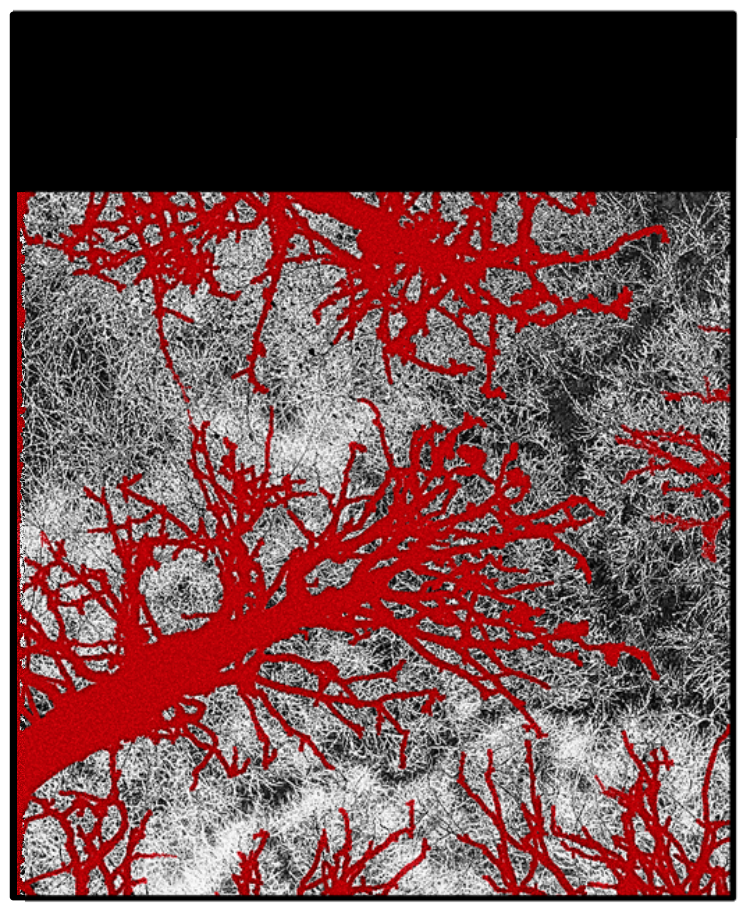


Samenvatting 
Hart- en vaatziekten zijn, met circa 17 miljoen sterfgevallen per jaar, één van de belangrijkste oorzaken van sterfte wereldwijd. De meest voorkomende hart- en vaatziekten zijn hartinfarcten en beroertes, die voor het overgrote deel veroorzaakt worden door het scheuren van atherosclerotische laesies.

Atherosclerose, in de volksmond beter bekend onder de naam aderverkalking, is een aandoening gekenmerkt door een chronische ontstekingsreactie in de vaatwand van de slagaders op plaatsen met een turbulent bloedstroomprofiel. Deze ontstekingsreactie ontstaat door het binnendringen van LDL cholesterol in de vaatwand doorheen de laag endotheelcellen, die de vaatwand bekleed. Na processen als oxidatie van het LDL cholesterol worden vervolgens signalen afgegeven waardoor monocyten worden aangetrokken tot de vaatwand. Na het binnendringen in de vaatwand differentiëren de monocyten tot macrofagen in een poging het geoxideerde LDL cholesterol te verwijderen uit de vaatwand. Echter, de opname van grote hoeveelheden geoxideerd LDL cholesterol door macrofagen zal ervoor zorgen dat deze veranderen in schuimcellen, die niet langer in staat zijn het LDL cholesterol af te breken. Daardoor vindt er een opstapeling plaats van schuimcellen en geoxideerd LDL cholesterol in de vaatwand. Dit is de stimulans voor een chronische ontstekingsreactie waarbij verschillende ontstekingscellen, zoals T-cellen, een rol spelen. Na verloop van tijd ontwikkelen de bovengenoemde, schuimcelrijke vroege laesies zich verder naar geavanceerde laesies. Deze laesies worden gekenmerkt door een grote kern gevuld met onder andere cholesterol en resten van dode ontstekingscellen (necrotische resten). Deze kern is omgeven door een kapsel van gladde spiercellen en bindweefsel, dat de kern gescheiden houdt van de bloedstroom. Dit kapsel is echter gevoelig voor afbraak, en zal na verloop van tijd dunner worden hetgeen leidt tot een instabiele atherosclerotische laesie. Uiteindelijk kan dit kapsel dan ook scheuren, waarbij de inhoud van de necrotische en vetrijke kern in contact komt met het bloed. Hierdoor zal er een bloedstolsel ontstaan dat een bloedvat gedeeltelijk of volledig kan afsluiten. Wanneer dit gebeurt in de kransslagaders of de halsslagaders kan dit leiden tot respectievelijk een hartinfarct of beroerte.

Tijdens de ontwikkeling van atherosclerotische laesies worden ook nieuwe kleine bloedvaatjes (microvaten) gevormd in en rond de laesie via een proces dat bekend staat als angiogenese. Het is bekend dat er in humane atherosclerotische laesies een duidelijke correlatie is tussen het 
ontwikkelingsstadium waarin een laesie zich bevindt en de expansie van de microvaten in de buitenste laag van het bloedvat (de adventitia) als ook in de laesie zelf. Aan deze microvaten in en rond de laesies is zowel een positieve als negatieve rol toegeschreven. Aan de ene kant wordt gesuggereerd dat de microvaten een rol zouden kunnen spelen in het verwijderen van cholesterol en ontstekingscellen uit de laesie. Aan de andere kant zijn microvaten in de laesie juist geassocieerd met een toename van cholesterol en ontstekingscellen in de laesie. Recent is verder ook aangetoond dat lymfevaatjes in en rond de atherosclerotische laesie expanderen in verder gevorderde stadia van laesie ontwikkeling als gevolg van het proces genaamd lymfevat-angiogenese. De rol die deze lymfevaatjes spelen is nochtans onduidelijk.

Daarom hebben we in dit proefschrift getracht meer duidelijkheid te krijgen over de rol die microvaten en lymfevaatjes spelen in de ontwikkeling van atherosclerotische laesies. De hypothese behorende bij dit proefschrift is dat angiogenese en lymfevat-angiogenese een belangrijke rol hebben in de ontwikkeling van atherosclerotische laesies, en dat afwijkende functie van microvaten en lymfevaten in en rondom de laesies bijdraagt aan verdere ontwikkeling hiervan.

De eerste studies in deze thesis (hoofdstukken 2-5) zijn gefocust op het bepalen van de functionele karakteristieken van microvaten rondom atherosclerotische laesies in levende dieren (in vivo). Hierbij hebben we gebruik gemaakt van multifoton microscopie, een op fluorescentie gebaseerde techniek waarmee het mogelijk is in vivo door weefsel te scannen. Hoofdstuk 2 geeft een overzicht van de huidige status van in vivo beeldvorming met behulp van multifoton microscopie, en bediscussieert de baten en tekortkomingen van deze techniek.

In hoofdstuk 3 is deze techniek vervolgens toegepast om microvaten rondom atherosclerotische laesies in een muismodel voor atherosclerose, de ApoE-deficiënte muis, te onderzoeken. Met behulp van deze techniek konden we microvaten rondom atherosclerotische laesies in beeld brengen en hieruit bleek dat deze microvaten in dit model specifiek voorkomen in muizen met vergevorderde laesies, maar niet in dieren zonder laesies of met vroege laesies. Rondom de atherosclerotische leasies kon actieve vorming van nieuwe microvaten (angiogenese) worden aangetoond, en deze microvaten vertoonden een verlaagde bloedstroomsnelheid, een verhoogde permeabiliteit 
(doorlaatbaarheid) en een versterkte rekrutering van ontstekingscellen in vergelijk met controle-microvaten. Deze functionele kenmerken kunnen gezien worden als bevorderend voor verdere ontwikkeling van atherosclerotische laesies.

Om een beter inzicht te krijgen in de mogelijke oorzaken van de bovengenoemde functionele kenmerken, werden hierna twee belangrijke adhesiemoleculen, die de endotheelcellen onder normale omstandigheden met elkaar verbinden, bestudeerd. In hoofdstuk 4 is gekeken naar de rol van het adhesiemolecuul JAM-A op permeabiliteit van de vaatwand en de rekrutering van ontstekingscellen. De resultaten lieten zien dat JAM-A, in ieder geval op de vaatwand van de slagaders zelf, van belang is voor adhesie van ontstekingscellen aan, en transmigratie door de vaatwand. Veranderde distributie van deze moleculen op de endotheelcellaag, zoals in atherosclerotische laesies, zorgt voor een versterkte rekrutering van ontstekingscellen. Er was geen effect op de permeabiliteit van de endotheelcellaag. In hoofdstuk 5 is vervolgens specifiek in de microvaten rondom de atherosclerotische laesies gekeken naar het molecuul VEcadherine. Wanneer de binding van VE-cadherine tussen de endotheelcellen werd verstoord met behulp van een specifiek antilichaam, werd in controlemicrovaten een versterkte permeabiliteit gemeten, als ook een versterkte transmigratie van ontstekingscellen door de vaatwand heen. Daarentegen was dit effect niet waarneembaar in microvaten rondom de atherosclerotische laesies. Deze bevindingen suggereren dat hetzij VE-cadherine al dysfunctioneel is in microvaten rondom atherosclerotische laesies en dus niet verder verstoord kan worden, hetzij VE-cadherine geen rol speelt in microvaten rondom atherosclerotische laesies.

In hoofdstuk 6 is vervolgens gekeken naar expressie van genen in humane atherosclerotische laesies in relatie tot de vorming van nieuwe microvaten en lymfevaten in de laesies. Hierbij is rekening gehouden met de veranderde expressie van genen tussen stabiele en instabiele geavanceerde laesies. Op basis van deze methode konden zestien genen en vijf micro-RNAs, betrokken bij genregulatie, gecorreleerd worden met de toename van microvaten in instabiele atherosclerotische laesies. Voor lymfevat-angiogenese konden in de eerste analyses geen genen gecorreleerd worden aan de toename van lymfevaten in instabiele atherosclerotische laesies. 
Aangezien de rol van lymfevat-angiogenese in de verdere ontwikkeling van atherosclerose niet bekend is, is in hoofdstuk 7 de rol van lymfevaten in atherosclerose in het ApoE-deficiënte muismodel bestudeerd. $\mathrm{Na}$ het verwijderen van de lymfeknopen die verbonden zijn met de lokale lymfevaten rondom atherosclerotische laesies, kon een versterkte laesie-ontwikkeling worden waargenomen, waarbij de grootte van de laesie toenam en er een groter aantal T-lymfocyten in en rond de laesie aanwezig was. Vergelijkbare resultaten, met een toename in het aantal T-cellen rondom de laesie, werden verkregen nadat specifiek de lymfevat-angiogenese werd geremd. Deze data suggereren dat lymfevat-angiogenese in latere stadia van de ontwikkeling van atherosclerotische laesies een mogelijk remmende werking heeft.

Tot slot werden in hoofdstuk 8 de resultaten beschreven in dit proefschrift en de implicaties hiervan bediscussieerd en in perspectief geplaatst en werden opties gegeven voor verder onderzoek. Concluderend, dit proefschrift geeft nieuwe inzichten in de rol van angiogenese en lymfevatangiogenese in de ontwikkeling van atherosclerose. 


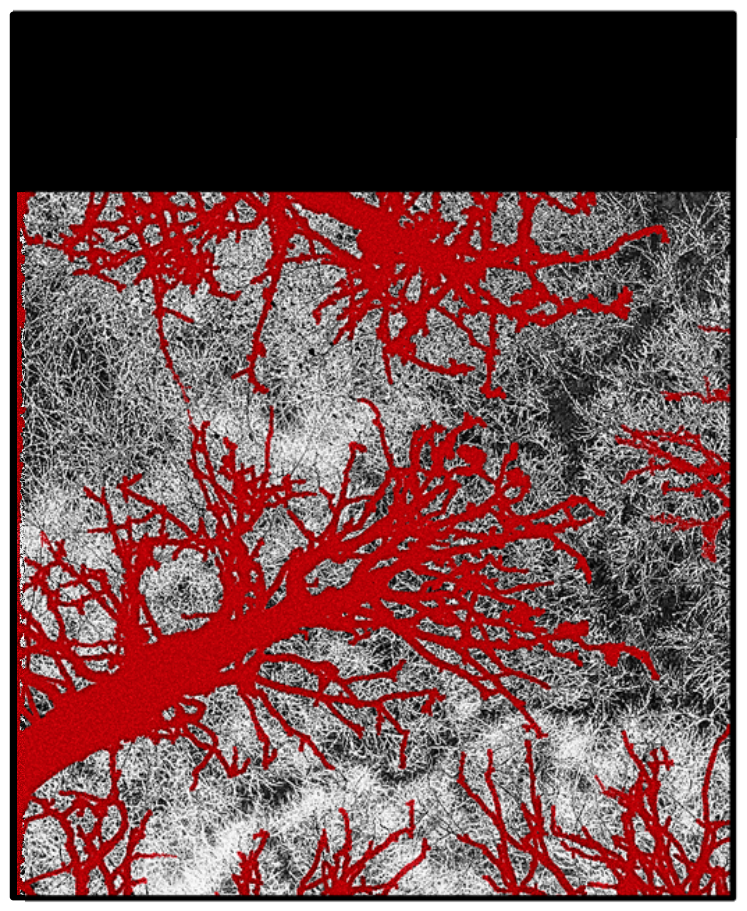




\section{Curriculum}

Vitae 
Personal details:

Name Timo Rademakers

Date of birth July 3, 1985

Place of birth Brunssum, The Netherlands

Nationality Dutch

\section{Education:}

August 2008 - November 2012

Maastricht University, departments of Pathology and Biomedical Engineering; PhD student

September 2006 - August 2008

Maastricht University/ transnational University Limburg; MSc Clinical Molecular Sciences, cum laude

September 2003 - August 2006

Maastricht University; BSc Molecular Life Sciences

September 1997 - June 2003

Trevianum Scholengroep Sittard; Gymnasium (Nature \& Health, Nature \& Technology)

\section{Grants and prizes:}

Best poster award for Vascular Biology \& Vascular Medicine $-13^{\text {th }}$ Dutch Atherosclerosis Society Symposium (Ede, NL)

2011

Young Investigators Travel Award - American Heart Association Scientific Session 2011, Council on Arteriosclerosis, Thrombosis, and Vascular Biology for Young Investigators (Orlanda, FL, USA)

\section{Publications:}

Wu Z, Rademakers T, Curaj A, Ghosh M, Kiessling F, Vogt M, Megens RTA, van Zandvoort MAMJ. Multiphoton Laser Scanning Microscopy in Atherosclerosis Research. Submitted. 
Schmitt MMN, Koenen RR, Megens RTA, Zernecke A, Rademakers T, Bidzhekov $\mathrm{K}$, van den Akker NM, Reichel CA, Krombach F, van Zandvoort MAMJ, Hackeng TM, Weber C. Cell-specific role of junctional adhesion molecule-A in flowdependent atherosclerosis. Submitted.

Rademakers T*, Douma K*, Hackeng TM, Post MJ, Sluimer JC, Daemen MJ, Biessen EAL, Heeneman S, van Zandvoort MAMJ. Plaque-associated vasa vasorum in aged apolipoprotein e-deficient mice exhibit proatherogenic functional features in vivo. Arterioscler Thromb Vasc Biol. 2013;33(2):249-56.

Lievens $\mathrm{D}$, Habets $\mathrm{KL}$, Robertson AK, Laouar $\mathrm{Y}$, Winkels $\mathrm{H}$, Rademakers $\mathrm{T}$, Beckers L, Wijnands E, Boon L, Mosaheb M, Ait-Oufella H, Mallat Z, Flavell RA, Rudling M, Binder CJ, Gerdes N, Biessen EA, Weber C, Daemen MJ, Kuiper J, Lutgens $\mathrm{E}$. Abrogated transforming growth factor beta receptor II (TGFbetaRII) signalling in dendritic cells promotes immune reactivity of $T$ cells resulting in enhanced atherosclerosis. European Heart Journal. 2012.

Nergiz-Unal R, Rademakers T, Cosemans JM, Heemskerk JW. CD36 as a multiple-ligand signaling receptor in atherothrombosis. Cardiovascular \& hematological agents in medicinal chemistry. $2011 ; 9(1): 42-55$.

Rademakers T*, Theelen TL*, van Buul JD, Cleutjens JP, van de Vijver P, Hackeng TM, van Zandvoort MAMJ, Daemen MJAP, Sluimer JC. VE-cadherin as a potential mediator of microvascular permeability in atherosclerosis?. In preparation.

Rademakers $\mathrm{T}^{*}$, Manca $\mathrm{M}$, van der Loos $\mathrm{CM}$, van $\operatorname{der}$ Wal $\mathrm{AC}$, van den Akker LH, Peutz-Kootstra CJ, van Zandvoort MAMJ, Heeneman S, Biessen EAL. Angiogenic and lymphangiogenic responses in atherosclerosis - A genomicsbased approach to identify new regulators of plaque (lymph)angiogenesis. In preparation

Daissormont ITMN, Rademakers T, Otten JJT, Anisimov A, Nurmi H, van Zandvoort MAMJ, Heeneman S, Alitalo K, Biessen EAL. Increased adventitial lymphatic capillaries in atherosclerosis: a path for $\mathrm{T}$ cell trafficking?. In preparation 


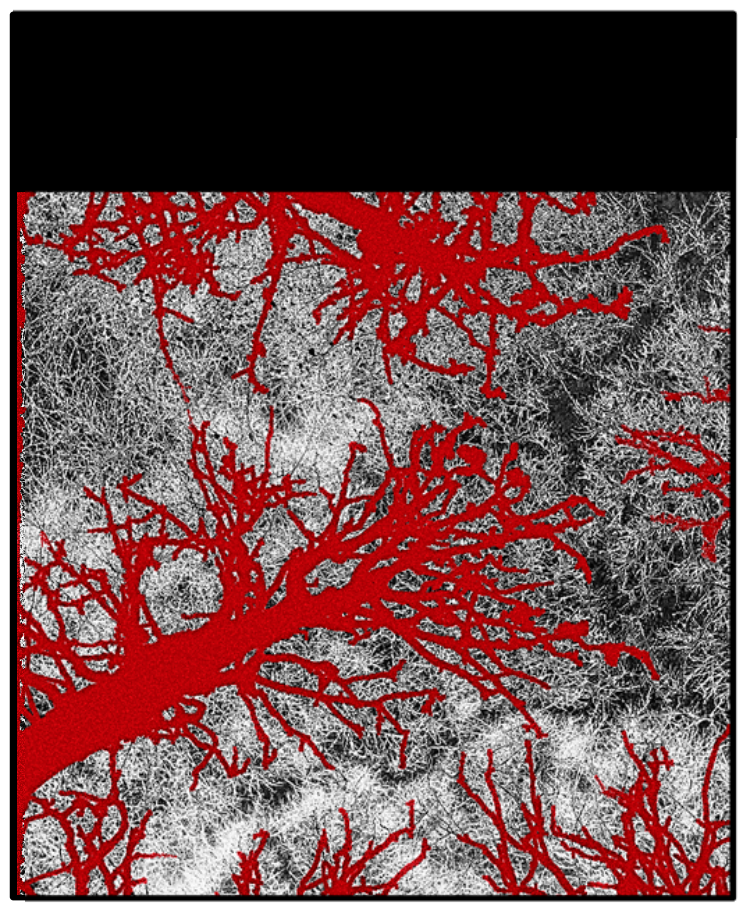


List of

abbreviations 


$\begin{array}{ll}\text { AAV } & \text { Adeno-associated virus } \\ \text { AMI } & \text { Acute myocardial infarction } \\ \text { Ang } & \text { Angiopoietin } \\ \text { ANOVA } & \text { Analysis of variance } \\ \text { ApoE } & \text { Apolipoprotein-E } \\ \text { aSMA } & \text { Alpha smooth muscle actin } \\ \text { CCD } & \text { Charge-coupled device } \\ \text { CCL } & \text { CC chemokine ligand } \\ \text { CCR } & \text { CC chemokine receptor } \\ \text { CD } & \text { Cluster of differentiation } \\ \text { CEUS } & \text { Contrast-enhanced ultrasound } \\ \text { CFSE } & \text { Carboxyfluorescein succinimidyl ester } \\ \text { Col18 } & \text { Collagen type XVIII } \\ \text { CT } & \text { Computed tomography } \\ \text { CVD } & \text { Cardiovascular disease } \\ \text { CXCR } & \text { Chemokine (C-X-C motif) receptor } \\ \text { DAB } & \text { 3,3'-Diaminobenzidine } \\ \text { DC } & \text { Dendritic cell } \\ \text { EC } & \text { Endothelial cell } \\ \text { ECIS } & \text { Electric cell-substrate impedance sensing } \\ \text { ECM } & \text { Extracellular matrix } \\ \text { EDTA } & \text { Ethylenediaminetetraacetic acid } \\ \text { ELISA } & \text { Enzyme-linked immunosorbent assay } \\ \text { EM } & \text { Electron microscopy } \\ \text { EVG } & \text { Elastica van Gieson } \\ \text { FACS } & \text { Fluorescence-activated cell sorting } \\ \text { FAD } & \text { Flavin adenine dinucleotide } \\ \text { FGF } & \text { Fibroblast growth factor } \\ \text { FITC } & \text { Fluorescein isothiocyanate } \\ \text { FLIM } & \text { Fluorescence-lifetime imaging microscopy } \\ \text { FMT } & \text { Fluorescence molecular tomography } \\ \text { Fox-C2 } & \text { Forkhead box protein C2 } \\ \text { fps } & \text { Frames per second } \\ \text { HAoEC } & \text { Human aortic endothelial cells } \\ \text { HBSS } & \text { HDL }\end{array}$




$\begin{array}{ll}\text { HE } & \text { Haemotoxylin and eosin } \\ \text { HIF } & \text { Hypoxia inducible factor } \\ \text { HUVEC } & \text { Human umbilical vein endothelial cell } \\ \text { i.p. } & \text { Intraperitoneal } \\ \text { i.v. } & \text { Intravenous } \\ \text { ICAM } & \text { Intercellular adhesion molecule } \\ \text { IFN } & \text { Interferon } \\ \text { IHC } & \text { Immunohistochemistry } \\ \text { IL } & \text { Interleukin } \\ \text { IMT } & \text { Intima-media thickness } \\ \text { IP-10 } & \text { Interferon gamma-induced protein 10 } \\ \text { IPH } & \text { Intraplaque hemorrhage } \\ \text { JAM-A } & \text { Junctional adhesion molecule A } \\ \text { KDa } & \text { Kilodalton } \\ \text { KLF } & \text { Kruppel-like factor } \\ \text { LDL } & \text { Low density lipoprotein } \\ \text { LDLr } & \text { Low density lipoprotein receptor } \\ \text { LEC } & \text { Lymphatic endothelial cell } \\ \text { LFA } & \text { Lymphocyte function-associated antigen } \\ \text { LN } & \text { Lymph node } \\ \text { LVD } & \text { Lymph vessel density } \\ \text { LYVE-1 } & \text { Lymphatic vessel endothelial hyaluronic acid receptor 1 } \\ \text { miRNA / miR } & \text { microRNA } \\ \text { MMP } & \text { Matrix metalloproteinase } \\ \text { MPLSM } & \text { Multiphoton laser scanning microscopy } \\ \text { MPTC } & \text { Maastricht Pathology Tissue Collection } \\ \text { MRI } & \text { Magnetic resonance imaging } \\ \text { mRNA } & \text { Messenger RNA } \\ \text { MVD } & \text { Microvessel density } \\ \text { NAD(P)H } & \text { Nicotinamide adenine dinucleotide phosphate } \\ \text { NF-KB } & \text { Nuclear factor kB } \\ \text { NIR } & \text { Near-infrared } \\ \text { NO } & \text { Nitric oxide } \\ \text { NRP } & \text { Neuropilin } \\ \text { OCT } & \text { Optical coherence tomography } \\ \text { OPO } & \text { Optical parametric oscillator } \\ & \end{array}$




$\begin{array}{ll}\text { ORO } & \text { Oil red O } \\ \text { OXLDL } & \text { Oxidized low density lipoprotein } \\ \text { PAT } & \text { Photoacoustic tomography } \\ \text { PBS } & \text { Phosphate buffered saline } \\ \text { PDGF } & \text { Platelet-derived growth factor } \\ \text { PDT } & \text { Photodynamic therapy } \\ \text { PE } & \text { Phycoerythrin } \\ \text { PECAM } & \text { Platelet endothelial cell adhesion molecule } \\ \text { PET } & \text { Positron emission tomography } \\ \text { PFA } & \text { Paraformaldehyde } \\ \text { PI } & \text { Propidium iodide } \\ \text { PIGF } & \text { Placental growth factor } \\ \text { PMT } & \text { Photo-multiplier tube } \\ \text { Prox-1 } & \text { Prospero homeobox 1 } \\ \text { PTT } & \text { Photothermal therapy } \\ \text { QD } & \text { Quantum dot } \\ \text { RNA } & \text { Ribonucleic acid } \\ \text { S.C. } & \text { Subcutaneous } \\ \text { SEM } & \text { Standard Error of the Mean } \\ \text { SH } & \text { Sternohyoid } \\ \text { SHG } & \text { Second harmonic generation } \\ \text { SPECT } & \text { Single-photon emission computed tomography } \\ \text { TBS } & \text { Tris-buffered saline } \\ \text { THG } & \text { Third harmonic generation } \\ \text { TkFCA } & \text { Thick fibrous cap atheroma } \\ \text { TLO } & \text { Tertiary lymphoid organ } \\ \text { TLR } & \text { Toll-like receptor } \\ \text { TMR } & \text { Tetramethylrhodamine } \\ \text { TNF } & \text { Tumor necrosis factor } \\ \text { TP } & \text { Two-photon } \\ \text { VALT } & \text { Vascular-associated lymphoid tissue } \\ \text { VCAM } & \text { Vascular cell adhesion protein } \\ \text { VE-cadherin } & \text { Vascular endothelial cadherin } \\ \text { VEGF } & \text { Vascular endothelial growth factor } \\ \text { VEGFR } & \text { Vascular endothelial growth factor receptor } \\ \text { VE-PTP } & \text { Vaschelial protein tyrosine phosphatase }\end{array}$




$\begin{array}{ll}\text { VSMC } & \text { Vascular smooth muscle cell } \\ \text { VV } & \text { Vasa vasorum } \\ \text { VWf } & \text { Von Willebrand factor } \\ \text { WGA } & \text { Wheat germ agglutinin } \\ \text { WT } & \text { Wild-type } \\ \text { WTD } & \text { Western type diet }\end{array}$




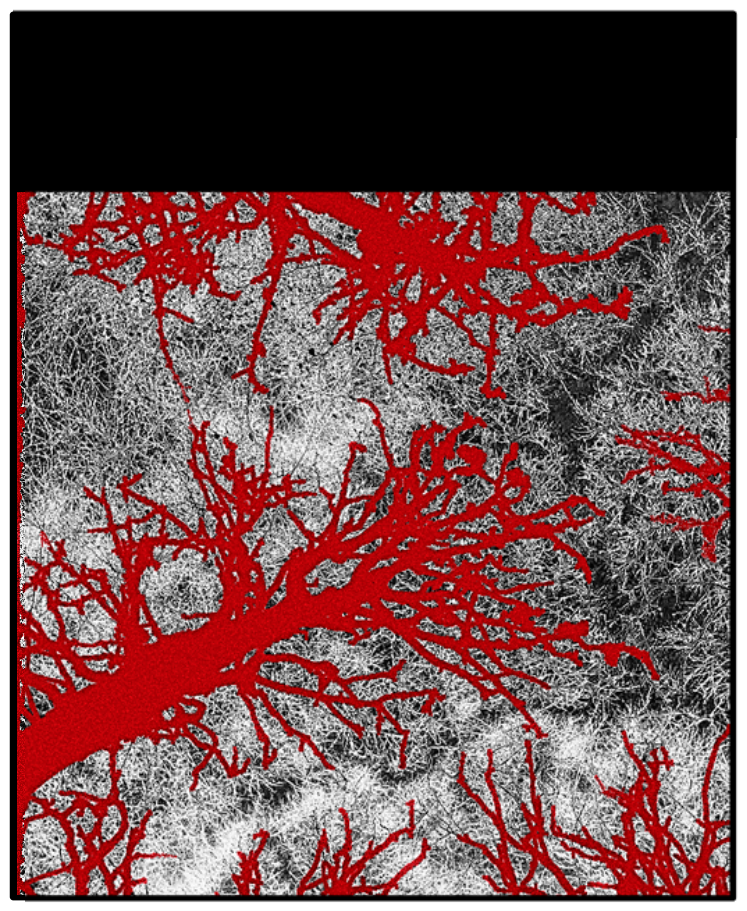


Dankwoord 
En na 233 pagina's (er komen nogal wat pagina's bij, dus ga er even voor zitten), volgt nu het misschien wel belangrijkste en in elk geval meest gelezen deel van het proefschrift: het dankwoord. Terecht een belangrijk deel, want ruim 4 jaar promoveren doe je uiteraard niet alleen. Op de eerste plaats ben ik heel veel dank verschuldigd aan mijn promotie-team!

Prof. Dr. Biessen, beste Erik. Ik herinner me nog als de dag van gisteren onze eerste werkbespreking: ik kwam helemaal murw van alle nieuwe termen en ideeën je kantoor uitgelopen, en realiseerde me dat er nog tientallen van deze besprekingen zouden volgen. Hoe langer ik bezig was, hoe gemakkelijker het werd om je ideeën te kunnen volgen, maar tot mijn verbazing bleef de vrijwel eindeloze stroom aan ideeën constant. Ik kan alleen maar ontzettend veel waardering uitspreken hiervoor, want er waren meer dan genoeg ideeën waar geen normaal mens op zou komen. Erik, ik wil je in ieder geval heel hartelijk bedanken voor de afgelopen 4,5 jaar. Ondanks je soms chaotische aard (die ook wel eens botste met mijn minder chaotische aard), heb ik veel dingen van je kunnen leren, dingen waar ik in de toekomst ook zeker profijt van zal hebben. Ik kijk er in ieder geval naar uit om nog invulling te kunnen geven aan afronding van een aantal van de studie uit dit proefschrift.

Prof. Dr. van Zandvoort, beste Marc. Jij was de imaging-man van het promotieteam! Met een onuitputtelijk optimisme verkondigde je 4,5 jaar geleden dat tweefoton-microscopie zo moeilijk niet was, en dat die plaquevaatjes vinden gewoon een kwestie was van aan wat knopjes en schuifjes zitten en goed zoeken (althans, dat heb ik ervan onthouden). Nu zou ik het toch niet als eenvoudig betitelen (zie ook stelling 6), maar het is uiteindelijk toch een erg succesvolle onderneming gebleken. Je optimistische houding was in elk geval een welkome afwisseling op de donkere en onheilspellende krochten van de tweefotonruimte, en zeker ook richting het einde van mijn promotie heb ik dat optimisme erg gewaardeerd. Het is jammer dat er op het moment geen imaging-avonturen in het verschiet liggen, maar wie weet wat de toekomst gaat brengen. Ik zal in elk geval nog geregeld bij MCB komen langswandelen voor een praatje.

(Bijna Prof.) Dr. Heeneman, beste Sylvia. Het rustpunt van het promotie-team en degene die een goede hulp was in het in goede banen leiden van de soms 
wilde ideeën van de heren promotores. Daarnaast ben je natuurlijk EVP's DECspecialist en dé persoon die volhardend mijn manuscripten corrigeert corrigeerde; mijn schrijfstijl zal nooit meer dezelfde zijn ;-). Het meest gedenkwaardig waren echter de niet-proefschrift-gerelateerde discussies. Wat meestal (semi-)serieus begon, eindigde meer dan eens met één van je gevatte en soms licht-dubieuze opmerkingen. Ik ben in elk geval blij dat ik nog even blijf plakken bij de pathologie en me nog mag verheugen op meer van dit soort discussies. Heel erg bedankt voor alle hulp!

Dr. Sluimer, beste Judith. Voordat ik overga tot het bedanken van alle anderen, verdien jij een extra bedankje. Want hoewel je niet officieel deel uitmaakte van mijn promotie-team, was je op zijn minst mijn co-co-promotor, en ik ben je dan ook erg dankbaar dat je je na je terugkeer uit New York aan hebt gesloten bij het angiogenese-clubje. Jouw expertise op dit gebied was een perfecte aanvulling op het promotie-team, en jouw proefschrift is ook vaak een goed naslagwerk voor mij geweest. Ik vond het ook erg leuk om samen met jou en Thomas te kunnen werken aan het VE-cadherine-stuk, waar we het komende jaar zeker nog een mooie publicatie uit gaan halen! Ik wens je alle goeds voor de toekomst, zowel professioneel, als ook met Steven en Sophie.

Naast een promotie-team, kan niemand zonder zijn paranifmen. Herr Doktor Engel, lieber David, was soll ich hier jetzt noch schreiben. Ich würde sagen, dass vieles schon mal gesagt wurde in den letzten Jahren. Ich kann nur sagen, dass jeder einen besten Freund wie dich haben sollte! Du bist immer da wenn es mal nötig sein sollte und hast fast immer gute Laune (auch wenn man urteilend nach deinem Gefluche im Büro manchmal anders behaupten würde). Ich bin dann wirklich sehr erfreut, dass wir 4 Jahre zusammen arbeiten konnten, und sogar Nachbarn waren im Büro. Außerhalb der Arbeit hatten wir wirklich ne Menge Spaß in Maastricht, Aachen, Ede, Hamburg, Biezenmortel (ja genau, noch immer weiß keiner wo das liegt), Stockholm, Noordwijkerhout, Florida oder Bonn. Ich bin jedenfalls froh, dass wir uns in Stockholm schon mal entschieden hatten gegenseitig Paranimfen zu sein, und ich freue mich, dass du mit dabei bist zur Promotion. Ich bin mir sicher, dass wir uns auch nach meiner Promotion immerhin noch oft sehen werden in Bonn oder Maastricht, und hoffe wir können in Zukunft auch noch mal einige witzige Sachen unternehmen (der Rekord von 25 Achterbahnfahrten war ja nur ein guter 
Anfang). Ich wünsche dir alles Gute für deine Zeit im Kreis Bonn (und Köln ;-)) und bis demnächst!

Beste Jeroen, wij kennen elkaar inmiddels bijna 10 jaar, met dank aan de introductiedag van MLW (jij was net als ik één van de enthousiastelingen die natuurlijk véél te vroeg aanwezig was). We zijn toen eigenlijk wel vanaf de eerste dag goede vrienden geworden, en naast de studie hebben we samen ook een aantal bestuursjaren bij Helix en Xist erop zitten. Na een kort uitstapje als CSI-trainee Otten, ben je toen toch weer teruggekomen naar het zuiden en kwam je na een tip van mij terecht bij EVP (sorry, je had inderdaad een veel normaler leven kunnen hebben ;-)). In de 4 jaren sindsdien heb ik altijd graag met je samengewerkt. Verder waren er ook de nog veel leukere dingen, zoals bezoeken van congressen (met als hoogtepunt het exotische Humlebæk en omgeving), of buiten werk bijvoorbeeld je bruiloft (of beter nog, het vrijgezellenfeest;-)). Inmiddels zit voor jou het promotietraject er ook bijna helemaal op. Ik wil je bij dezen heel veel succes wensen bij het afronden van je eigen boekje en het zoeken van een nieuwe baan, en uiteraard heel veel geluk samen met Stefanie en Finn!

Zo, hiermee zijn we aangekomen bij de rest van de EVP'ers. Liebe Anette, wir haben fast zur gleichen Zeit angefangen und du bist mittlerweile auch mit deinem Buch fertig. Ich freu mich jedenfalls schon mal auf deiner Promotion, die wird sicher super, wie auch die Party nachher. Weil, wie ich schon mal gesagt habe, du bist ja heimlich ne richtige Party-Tigerin ;-). Es war deswegen dann auch extrem witzig als wir mit der deutsche Truppe unterwegs waren in Maastricht oder Aachen. Ich wünsch dir für jetzt viel Erfolg und Glück in die Staaten. Bis bald!

Beste Karen, ook jij bent er inmiddels al een paar jaartjes bij. Ooit hier begonnen als onderdeel van pathologie's eigen K3 (ja, lang geleden, en ik zou er eigenlijk niks meer over zeggen geloof ik, oeps), en vervolgens hier gebleven om te promoveren. Jij bent toch ook wel één van de schoolvoorbeelden van de (te?) lieve Belgische meisjes. Ik heb in ieder geval graag met je samengewerkt, en erg leuk ook dat je door je Kootstra nu nog wat langer hier kunt blijven. Jij ook succes met het afronden van je promotie, nog een feestje waar we binnenkort naar uit kunnen kijken, en heel veel geluk samen met Wim. 
Beste Lieve (ofwel lieve dr. Lieve), sinds een jaartje of 2 maak je ook deel uit van de groep. De altijd drukke maar toch immer vrolijke Belg die terugkwam vanuit Italië. Ik denk dat je je gelijk wel thuis hebt gevoeld in de groep, en je was (samen met Lucas :D) ook direct altijd van de partij als er weer iets te vieren viel. Dit heeft in ieder geval ook een erg geslaagde patho-bingo-borrel bij jou thuis opgeleverd. Ik hoop dat je nog een tijd bij de EVP'ers zult blijven, want je kritische blik en goede ideeën zijn een erg waardevolle toevoeging. Ik wens je heel veel succes met al je projecten en veel geluk thuis met Pieter, Lucas, en ... :-).

Ine, na je transfer vanuit Molgen ben je ook op de AIO-kamer van de EVP terechtgekomen. Niet altijd zonder ergernis geloof ik, maar we zijn nu eenmaal fan van muziek en het nodige geklets op onze kamer ;-). Ik hoop dat je een leuke tijd hebt gehad bij die rare EVP'ers, je paste in ieder geval prima bij $;$.; Jij ook succes met de laatste loodjes van je promotie, de feestjes-teller staat dus op 4.

Thomas, sinds iets meer dan een jaar mijn patho-partner in crime bij de tweefoton. Ik ben blij dat ik in ieder geval iemand ook heb weten te enthousiasmeren voor het doen van in vivo proeven op de tweefoton. Dat heeft de nodige lange dagen opgeleverd, maar we hebben er toch mooie data uit kunnen slepen. Heel veel succes met je projecten, ik ben heel erg benieuwd wat er allemaal uit gaat komen. Verder ook heel veel succes met alle borrels die je in de komende jaren nog van plan bent te organiseren (toch?) en groetjes aan Anna!

Bart, net als ik ben je na een stage weer teruggekomen bij de pathologie als AIO. Altijd vroeg op werk, en altijd vriendelijk, en altijd hulpbeleid. Helaas heb je dan toch meer interesse in DCs dan vaatjes, maar goed, ieder z'n hobby ;-)... Heel veel succes de komende 2 jaar en veel geluk samen met Noor!

Elke, je bent de Benjamina van de groep, maar een goede aanvulling op de Deutsche Truppe. Je bent meestal erg duidelijk aanwezig (toch misschien al die koffie? ;-)), maar daarmee ben je ook een erg gezellig nieuwe buurvrouw op de kamer. En ik ben in ieder geval blij dat ik het zo vertrouwde Duitse gevloek 
naast me (ja herr Dr. Engel) niet meer hoef te missen. Heel veel succes in de komende jaren, ik weet zeker dat je wat moois weet te maken van je project!

Wat ben je verder op een lab, als je niet kunt rekenen op de hulp van een team ervaren en bovenal gezellige en praatgrage analisten. Anique, onze immunovraagbaak (en bedreven cupcake-bakster (;)). Hoe vaak hebben we in de afgelopen jaren niet het een of andere protocol bediscussieerd, of de kwaliteit van.... de cupcakes inderdaad. Ik houd me in elk geval aanbevolen voor als je nieuwe recepten gaat testen;-).

Clairy, jouw hulp bij genotyperingen, het doen van kleuringen en het werk voor de plaque-database van de EVP zijn van groot belang voor de groep. Bedankt voor je hulp de afgelopen jaren.

Erwin, de FACS-goeroe. Gezien mijn relatieve 'gebrek' aan FACS-experimenten hebben we op dat punt niet heel veel samengewerkt, maar je bent altijd in voor een borrel of feestje. Met je jarenlange ervaring en je grote interesse in nieuwe ontwikkeling op het gebied van flow cytometrie. ben je uitermate goed op de hoogte van allerlei manieren om de meest gekke dingen te FACSen. Ik heb nog wel wat FACS-plannen voor de toekomst, dus ik zal je nog wel een aantal keren komen lastigvallen. Groetjes ook aan Linda!

Mat, je hulp op het gebied van kweken en de moleculaire technieken was erg waardevol. Alhoewel ik de afgelopen jaren maar relatief weinig met je heb samengewerkt op dat gebied, heb ik ook hier nog wel wat plannen, en ik kijk er dan ook naar uit om in de toekomst meer samen te kunnen werken.

Zo, nog een paar EVP'ers te gaan. Jack, mijn overbuurman op de afdeling. Wat zouden we moeten doen zonder je quantimet-programma's. Bedankt voor al je hulp en de vele discussies over microscopie, onderwijs, de patho-website, en het recept voor "hemelse modder".

Beste Marion, dank voor al je hulp bij de lastige histologische gevallen. Zeker op het collageen-XVIII project heb je me goed daarbij geholpen, jammer genoeg heeft dit project het niet tot het boekje gered. 
Danielle, jou heb ik toch wel oneindig vaak lastiggevallen met het inplannen van de zoveelste (verschoven) afspraak met mijn promotie-team of wat dan ook. Dank voor al je hulp in het oerwoud van afspraken plannen, en natuurlijk de gezellige praatjes op het secretariaat samen met Ingrid en Audrey.

Marjo, Nadine en Emiel. Hoewel jullie technisch gezien misschien maar halfEVPers zijn, horen jullie ook zeker bij de groep. Marjo, op de een of andere manier blijf je met je ADAMs-family toch telkens weer terugkomen bij de pathologie. Bedankt voor je goede suggesties en interesse in mijn project. Voor de toekomst waren er wel al wat leuke ideetjes, maar eens zien of we daar verder mee kunnen gaan.

Nadine, mijn maatje uit het angiogenese-portfolio, we hebben in die 4 jaar toch wel leuke data weten te verzamelen over angiogenese. Behalve de gezamenlijke interesse in angiogenese, zal je bij mij altijd bekend staan om je geweldig gevoel voor humor. Ik kan me genoeg melige momenten herinneren. Heel veel succes met het afronden van je eigen boekje, en het vinden van een nieuwe baan!

Emiel, jou ken ik natuurlijk met name van I'MCARIM, waar ik met veel plezier met je hebt samengewerkt. Je hebt verder een leuk (en soms verwarrend) project, maar daar weet je zeker wat van te maken!

Beste Carine, Floor en Petra. Jullie ben ik uiteraard niet vergeten, maar een apart stukje was wel op zijn plaats. Carine, bij jou is mijn verblijf bij de pathologie begonnen. Lang lang geleden kwam ik in de bachelor voor een korte stage bij jou bij de pathologie. Na niet al te lange tijd kwam ik alweer terug voor mijn afstudeerstage, en ben sindsdien ook niet meer weggegaan. Ik ben je in ieder geval erg dankbaar voor de goede indruk die je van de pathologie en onderzoek doen hebt achtergelaten en je interesse in mijn projecten gedurende de jaren. Het is in ieder geval erg leuk dat we het project dat we ooit gestart zijn al die jaren geleden nu alsnog kunnen afmaken en ik hoop dat dat slechts het begin is van vele andere projecten naar afwijkende microvaatjes in de nier. 
Beste Floor, ook jij bent inmiddels al jaren een bekend gezicht bij de pathologie. Je combinatie van geneeskunde afmaken en tegelijk onderzoek doen en promoveren was niet altijd even makkelijk, en je hebt dan ook de nodige erg lange dagen met SPSS gehad. Je bent een erg gezellige en leuke collega, en gelukkig zijn we het laatste jaar toch weer kamergenoten geworden. Hoewel het in dit dankwoord langzaamaan wel cliché begint te worden, wens ik ook jou heel veel succes met de afronding van je promotie. Je hebt genoeg mooie hoofdstukken, dus op naar promotiefeest nummer 6 . Heel veel succes alvast bij je specialisatie en veel geluk samen met Bas.

Petra, na mijn terugkomst bij de "niergroep", hebben we redelijk intensief samengewerkt de laatste maanden. Zonder jou waren we met de verschillende projecten nooit zo ver geweest, ook al levert al dat PCR en vaatjes tellen wel eens de nodige frustratie op $(-)$. Bedankt voor de prettige en gezellige samenwerking.

Verder een bedankje voor alle andere (oud)-EVP'ers voor hun praktische hulp, ideeën, handige suggesties, en/of gewoon gezelligheid en collegialiteit. Jasper, Kitty, Anjana, Marco, Sofia, Isabelle, Lili, Cora, Mathijs, Matthijs, Wouter, Dirk, Indira, Beatriz, Brenda, Marjorie, Veronica, Chantal, Barry, Jeffrey en Mirte, thank you all very much!

Een aantal oud-EVPers heeft tussendoor de overstap gemaakt naar Amsterdam, maar mogen zeker niet vergeten worden. Beste Linda, ik hoop dat je je wel eens hebt gerealiseerd, wat je vertrek heeft aangericht? Alle onze muisexperimenten liepen opeens totaal in de soep en er waren geen brownies en chocolate cookies meer! Nou is het misschien een heel klein beetje overdreven, en heeft het ons allen wel van onze (door jou geïnduceerde?) verslaving voor brownies en chocolate cookies geholpen, maar jij was toch wel degene bij wie iedereen te pas en te onpas kwam om iets te vragen of gewoon voor een praatje. Ik ben in ieder geval blij dat je het in Amsterdam/Vleuten goed naar je zin hebt, maar ik hoop dat je binnenkort weer eens in Maastricht langskomt! Heel erg bedankt voor alles, heel veel geluk in de toekomst en groetjes aan Lauran en Ella. 
Tom, toekomstig dr. dokter Seijkens. Je was altijd op het lab, en dan bedoel ik dus ook letterlijk altijd, tenzij je in de kliniek was. Je was een erg leuke collega, en ik heb me meer dan eens helemaal krom gelachen over je briljante, bijzondere en/of bedenkelijke uitspraken. Heel veel succes met het afronden van je projecten, het kan niet anders dan een heel mooi proefschrift worden.

Prof. Dr. Lutgens, beste Esther, hoe doe je toch alles wat je op een dag doet? Zelfs nu dat je al een tijdje in Amsterdam zit, heb ik dit raadsel nog niet kunnen oplossen. Hoe dan ook, ik wil je heel erg bedanken voor je goede suggesties, en heel veel succes verder in Amsterdam.

Prof. Dr. Daemen, beste Mat, bedankt voor je altijd kritische blik, maar zeker ook je blijvende interesse in het angiogenese-project. Zelfs na je vertrek naar Amsterdam bleef je graag op de hoogte over wat we aan het uitspoken waren in Maastricht, en je maakte dan ook elke keer dat ik eens in Amsterdam was tijd om ge-update te worden. Verder ook bedankt voor de prettige samenwerking bij CARIM gedurende de eerste jaren, en je steun bij de ideeën die we vanuit de AIO-vertegenwoordiging naar voren brachten.

Verder hebben de laatste jaar ook de nodige studenten de revue gepasseerd. Judith, Jan-Jurre, Bart, Laura, Sanne, Bert, Marnix en Romy, bedankt voor jullie harde werk de afgelopen jaren op de verschillende projecten en de leuke tijd tijdens jullie stages!

Daarmee zijn we aanbeland bij de rest van de pathologie, want de afdeling pathologie is meer dan EVP alleen. Ten eerste de "epi's". Beste Manon, Veerle, Hanneke, Joep, Karin, Kim W, Edith, Peter, Jürgen, Kim S, Iris, Suzanne, Ingrid en oud-epi's die ik zou zijn vergeten: bedankt voor de leuke discussies, gesprekken en soms ook samenwerkingen. Altijd leuk om weer eens over wat anders te praten dat athero;-). Also the people from Axel's group, thank you for the being nice colleagues!

En dan is er nog "de kliniek". Prof. Dr. Zur Hausen, beste Axel, dank voor de interesse in de projecten, ondanks dat deze mijlenver verwijderd zijn van virale oncologie. Cor \& Elly, jullie deur heb ik ook platgelopen zo nu en dan. Bedankt voor alle hulp gedurende de laatste jaren, en de gezellige gesprekken die daar 
meestal op volgden (ook als jullie daar geen tijd voor hadden). Dan een speciaal bedankje voor Unit IV, en dan met name Guido, Andrea, Jolanda en Margriet. Bij jullie werd ik zo'n 7 jaar geleden ondergebracht tijdens mijn eerste, en later ook tweede, stage. Bedankt voor de gezellige tijd daar op jullie lab en al jullie hulp tijdens de stages; de leuke tijd op jullie lab heeft er zeker aan bijgedragen dat ik wat langer ben blijven hangen bij de pathologie. Verder tot slot een bedankje aan de rest van de medewerkers van de afdeling die ik helaas niet allemaal bij naam kan gaan noemen. Heel erg bedankt allemaal voor de interesse, hulp en small talk in de wandelgangen; ik blijf met plezier nog wat langer plakken!

Zo... Voor degenen die nog wakker zijn tegen de tijd dat ze hier zijn aanbeland, ga ik nu over van pathologie naar een andere groep die zeker niet vergeten mag worden, mijn derde thuis, de tweefoton-groep. Beste Kim, jij was degene die ooit een keer bedacht had om te proberen plaque-vaatjes te imagen met behulp van tweefoton-microscopie. Deze data hebben een belangrijke rol gehad, zeker in de beginfase van mijn promotie. Ik heb in die tijd ook waanzinnig veel gehad aan je praktische ervaring om mij te leren tweefotonnen. Ik ben in ieder geval ook heel blij dat we nog een lange tijd samen hebben kunnen werken, en je hulp bij het schrijven en submitten en herschrijven en hersubmitten en herschrijven en... (ja, je snapt hem wel), was geweldig. Dank dus voor alles, heel veel succes met je opleiding en groeten aan Douwe en Marelle!

Beste Wim, hoe vaak is jouw naam niet over het tweefoton-lab geroepen als de apparatuur toch weer even niet deed wat het zou moeten doen? Jouw technische expertise was onontbeerlijk. Ik heb altijd met veel plezier met je samengewerkt, van de soms eindeloos durende practica, tot de borrelmomenten daarna bij Thembi. Geniet in ieder geval van je welverdiende pensioen, en laat maar weten als je weer eens komt buurten bij BME.

Lenneke, ook jij was één van de originele tweefotonners. Ook jij hebt me in de beginperiode altijd goed op weg geholpen, en was altijd in voor een praatje. Bedankt ook voor de blijvende interesse in het project, zelfs nadat je je opleiding tot klinisch chemicus was gestart. Deze zit er inmiddels ook alweer bijna op, dus heel veel succes met de laatste loodjes! 
Sietze, via een omweg is mijn project uiteindelijk ook een keer uitgekomen bij het kijken naar glycocalyx. Ik was dan ook erg blij dat we iemand hadden met ervaring op dat gebied, en je review heeft daarbij veel helderheid gebracht. Ondanks de combinatie van co-schappen en promoveren, maakte je altijd tijd om te helpen als dat nodig was. Bedankt en heel veel succes bij KNO!

Remco, wij kenden elkaar al van voor mijn promotie-traject, als ik het me goed herinner van toen ik nog stage liep bij de groep van Johan. Ik ben blij dat we nog geregeld samen hebben kunnen werken, eerst vooral ook praktisch in Aken, maar naderhand na je verhuizing naar München met name ook aan het review, etc.. Bedankt voor je hulp en heel veel succes nog in München, ik beloof dat ik echt nog een keer kom kijken naar de TP-opstellingen daar ;-).

Mitrajit, in the meantime you have already finished your project and are finishing your promotion as well. I've always been amazed by the way you kept on going with the NO project, I'm sure it would have driven me insane somewhere along the road. Thanks for the nice conversations and your kind nature, and good luck on the new job!

Anneke, hoewel je in de afgelopen jaren wegens persoonlijke omstandigheden vaak ook afwezig was, was je een ontzettend leuke collega. We zijn er helaas nog niet aan toegekomen om een keer ervaringen uit te wisselen op het gebied van bakken, moeten we binnenkort toch echt maar eens doen!

Jeroen ( $\mathrm{H}$.), onze nieuwe technische specialist voor de tweefoton. Wat ben ik blij dat we na het pensioen van Wim jou als nieuwe aanvulling op het TP-team kregen. Bedankt voor alle support, maar zeker ook de geslaagde practica en gezellige gesprekken!

Door de vele tweefoton-uren was ik ook veel te vinden bij de vakgroep die ooit nog biofysica heette, maar nu bekend staat als BME. Tammo, Koen, Joost, Wilco, Yvette, Bart, Jort, Claire, en alle anderen, heel erg bedankt voor de filmavonden, en in het algemeen voor alle gezelligheid. Ondanks dat ik maar de helft van de tijd bij BME te vinden was, heb ik me daar ook altijd thuis gevoeld! 
Wat recenter is er dan nog een extra vakgroep bijgekomen, de moleculaire celbiologie. Hoewel ik daar praktisch gezien niet gewerkt heb, was ik direct welkom om mee te doen met allerlei activiteiten. Bedankt allemaal!

Vervolgens zijn er nog meer UM'ers die een bedankje verdienen. Mark Post, Miriam, Menno, Johan, bedankt voor jullie kritische blik binnen het angiogenese-portfolio, de projecten hebben er veel voordeel bij gehad. Tilman, bedankt voor je hulp bij de verschillende hoofdstukken. Viviane, bedankt voor alle nuttige tips voor het in vivo werk; zoals je ziet, heb ik er voor dit boekje genoeg gebruik van gemaakt.

Marijke en Judith C, na mijn junior-stage ben ik nog vaak bij biochemie binnen komen vallen, gewoon voor een leuk gesprek (of omdat de tweefoton weer eens geen zin had). Leuk dat jullie altijd interesse zijn blijven houden, ik wandel binnenkort wel weer eens langs. Erik W, jammer dat je weer Down Under bent, maar bedankt voor de interessant discussies over data-analyse van de tweefoton-beelden; deze hebben veel inspiratie opgeleverd!

Verder verdienen ook de medewerkers van het CPV een woord van dank. Allen, maar in het bijzonder Richard, Paulien, Rik, Clarice, Mandy, Sytske en Saskia, bedankt voor alle goede zorgen voor de dieren de afgelopen jaren en de prettige samenwerking.

Over samenwerkingen gesproken, ook buiten de UM werd er met genoeg samengewerkt. Allard en Chris, bedankt voor jullie hulp in Amsterdam met de multi-spectraalanalyses. Ik ben altijd met veel plezier richting noorden gereisd.

Martin, es freut mich, dass es mit der JAM-A Versuche doch noch funktioniert hat. War anfangs noch nicht mal so einfach, aber die Daten waren umso besser! Viel Glück beim Zusammenschreiben deines Buches! Rory, jij ook bedankt voor je hulp en de prettige samenwerking.

Michael, Wu, and Adelina, thanks for the nice discussions about two-photon in Aachen. Wu, good luck with your project, I hope you'll get some nice in vivo data! 
David V. from Finland, thanks for the nice collaboration on the collagen-XV and $-X V I I I$ projects, the data look really promising. Good luck finishing up your own thesis!

Dan is er ook nog een leven buiten het onderzoek (ja, echt). Beste mensen bij CARIM (Riet, Esther, Johanna, Saskia, Tara, Petra, Rob en de rest), heel erg bedankt voor de leuke tijd die ik bij jullie heb gehad tijdens mijn periode als AIO-vertegenwoordiger. Ook iedereen die bij I'MCARIM heeft gezeten (Mathijs, Auke, Kelly, Romy, Ellen, Emiel, Yvonne, Siamack) bedankt!

Alle vrienden, oud-studiegenoten, en bekenden, heel erg bedankt voor de leuke tijd de afgelopen jaren, in het bijzonder Rianne, Roger, Ryanne, Marjolein, Jim, Dennis en Jo. Jennifer en Daniël; erg jammer dat jullie er niet bij kunnen zijn op de promotie, maar Canada is toch net ietsje te ver weg. Jen, zoals je zelf al refereerde, de reguliere koffiepauzes zijn wel een gemis. Heel veel succes en plezier daar en tot in oktober!

With the list of people who I should thank growing larger and larger, I'm sure I will have inadvertently forgotten. So, also to these people: thank you for your practical help, suggestions, kind words, support and/or "gezelligheid" during the last years.

Beste ooms en tantes, neven en nichten, hoewel we elkaar niet heel regelmatig zien, hebben jullie altijd de grootste interesse getoond in mijn studie en promotie. Ik wil jullie hiervoor dan ook heel erg bedanken en hoop dat in elk geval de Nederlandse samenvatting een beetje uitleg geeft van wat ik nu eigenlijk gedaan heb al die jaren!

Cody en Artan, ik weet dat jullie dit zelf niet kunnen lezen, maar dat betekent niet dat jullie niet ook een klein bedankje hier verdienen. Want toegegeven, als er 2 hondjes zijn die iemand in een klap kunnen opvrolijken, dan zijn jullie 2 het wel met jullie altijd vrolijke begroetingen en rare acties. Een hele dikke knuffel en aai over de bol voor jullie! 
Beste Glenn, je was vooraf bang dat ik je zou overslaan in het dankwoord, en hoewel het op zich best een grap was geweest om je gelijk te bewijzen, weet je net zo goed dat ik je niet zou overslaan. Mijn "kleine" (maar wel een kop groter zijnde) broertje, heel erg bedankt voor de gezelligheid thuis, de flauwe opmerkingen over en weer, de game-avonturen, etc.. Grappig genoeg bleek al snel dat ook jij de biomedische richting wel interessant vond, dus wie weet dat ik over een paar jaar jouw proefschrift tegemoet mag zien. Heel veel succes in ieder geval eerst met het halen van je bachelor (en master ;-)).

Beste pap en mam, zoals vaker wordt gezegd, bewaard men "the best for last", en dat is in dit geval zeker zo. Daarmee is deze laatste alinea van het boekje dan ook direct de moeilijkste om te schrijven, want ik denk dat niemand hier echt zijn ouders genoeg kan bedanken. Ik weet dat jullie ook met spanning hebben uitgekeken naar het boekje en de verdediging. Hopelijk helpen zowel het boekje als de verdediging om duidelijk te maken wat ik nu eigenlijk heb uitgespookt de laatste 4,5 jaar. Jullie hebben me in elk geval altijd mijn gang laten gaan en me in alles gesteund. Daarentegen kan ik altijd bij jullie terecht als ik ergens mee zit, of gewoon voor een weekendje "Hotel pap \& mam", om even te relaxen. Heel, heel, heel erg bedankt voor de onvoorwaardelijke steun, hulp, interesse en liefde in de afgelopen 28 jaar!

Timo 
